Supporting Information for

\title{
Visible-Light-Driven Bisfunctionalization of Unactivated Olefins via the Merger of PCET and Carbene Catalysis
}

\author{
Bei Zhang, Jian-Qing qi, Yuhan Liu, Zhipeng Li and Jian Wang* \\ School of Pharmaceutical Sciences, Key Laboratory of Bioorganic Phosphorous Chemistry \& \\ Chemical Biology (Ministry of Education), \\ Tsinghua University, Beijing, 100084, China. \\ E-mail: wangjian2012@tsinghua.edu.cn
}

\section{Contents}

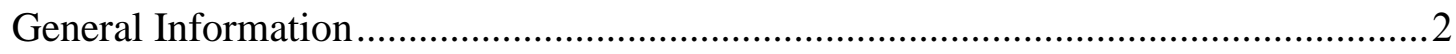

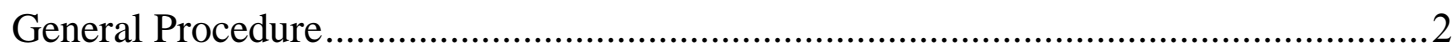

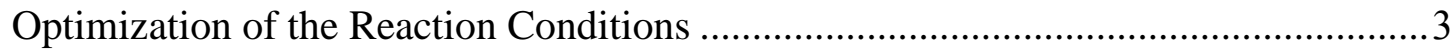

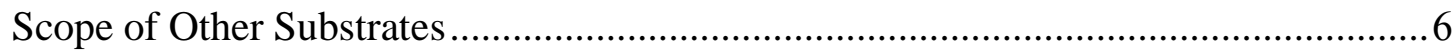

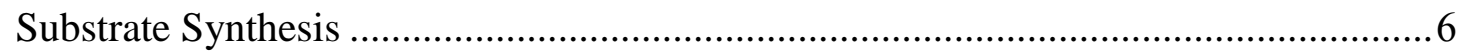

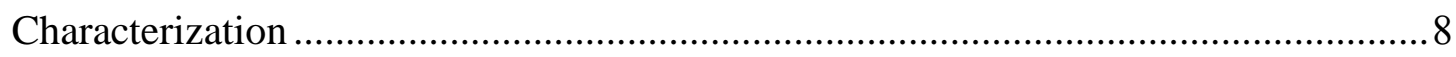

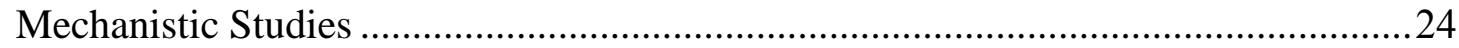

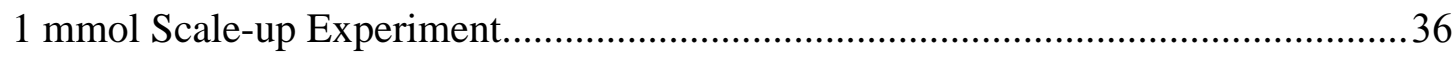

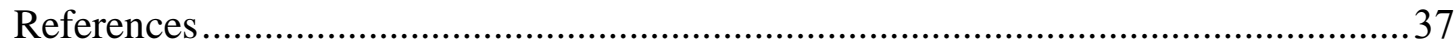

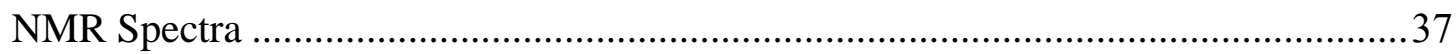




\section{General Information}

Chemicals were purchased from commercial suppliers and used as received. Solvents were dried on alumina columns using a solvent dispensing system. Thin-layer chromatography (TLC) was conducted on plates (GF254) supplied by Yantai Chemicals (China) and visualized using a combination of UV, anisaldehyde, iodine, and potassium permanganate staining. ${ }^{1} \mathrm{H}$ NMR, ${ }^{13} \mathrm{C}$ NMR, ${ }^{19} \mathrm{~F}$ NMR, spectra were recorded on a Bruker ACF400 (400 MHz) spectrometer. Chemical shifts were reported in parts per million ( $\mathrm{ppm})$, and the residual solvent peak was used as an internal reference: proton (chloroform $\delta 7.26, \mathrm{DMSO}_{-} \mathrm{d}_{6} \delta 2.50$ ), carbon (chloroform $\delta$ 77.16, DMSO- $\mathrm{d}_{6} \delta 39.52$ ) or tetramethylsilane (TMS $\delta 0.00$ ) was used as a reference. Multiplicity was indicated as follows: s (singlet), d (doublet), t (triplet), q (quartet), $\mathrm{m}$ (multiplet), dd (doublet of doublet), bs (broad singlet). Coupling constants were reported in Hertz $(\mathrm{Hz})$. All high resolution mass spectra were obtained from the Tsinghua University Mass Spectrometry Facility. Flash chromatography separations were performed on Silica gel (300-400 mesh) supplied by Tsingdao Haiyang Chemicals (China). Aldehydes 1a-1p, olefins 2a-2g, 2i, 2m were purchased from J\&K Scientific and used without further purification. Diazoesters 3a, 3c were purchased from energy chemical and used as received. Light source: 450-455nm 30W blue light barrel which was purchased from www.taobao.com. Irradiation vessel was made of common borosilicate glass without any filters.

\section{General Procedure}

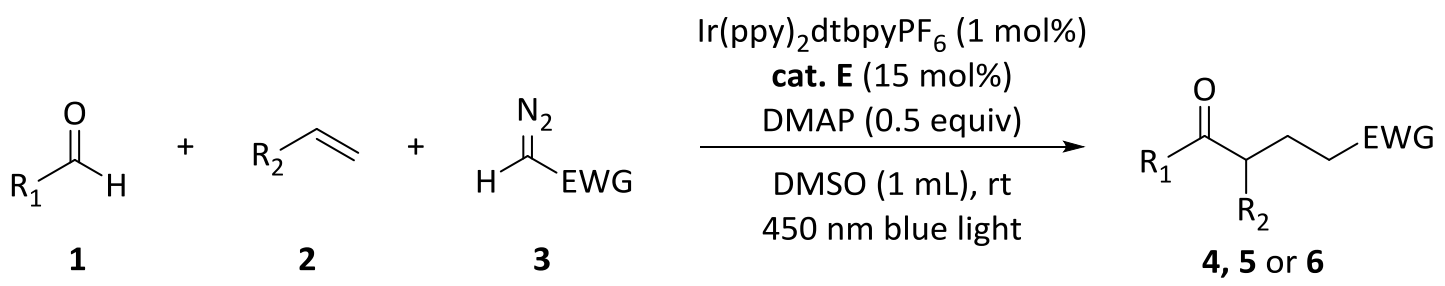

All the catalytic reactions were operated in a glovebox. To a flame-dried Schlenk reaction tube equipped with a magnetic stir bar, was added the precatalyst $\mathbf{E}$ (12 $\mathrm{mg}$, 0.03 mmol, 0.15 equiv), DMAP (12 mg, 0.22 mmol, 0.5 equiv), $\operatorname{Ir}(\mathrm{ppy})_{2} \mathrm{dtbpyPF}_{6}(2.0$ mg, 0.002 mmol, 0.01 equiv) and DMSO (2 mL, 0.1M). 1 (0.24 mmol, 1.2 equiv), 2 
( $0.2 \mathrm{mmol}, 1.0$ equiv), 3 ( $0.4 \mathrm{mmol}, 2.0$ equiv) were added subsequently. Then, the tube was caped and taken out of the glovebox. The resulting solution was irradiated by a $30 \mathrm{~W}$ blue LED $(450 \mathrm{~nm}$ ) with stirring at a distance of $3 \mathrm{~cm}$ (with cooling by the fan) at $25{ }^{\circ} \mathrm{C}$ for about $5 \mathrm{~h}$ until the styrene was consumed completely, as monitored by TLC analysis. The mixture was diluted with EtOAc, washed with water and dried by $\mathrm{Na}_{2} \mathrm{SO}_{4}$. The organic phase was then concentrated under reduced pressure and purified by column chromatography on silica gel (Petroleum Ether/EtOAc) to afford the desired product $\mathbf{4}$ or 5 or $\mathbf{6}$.

\section{Optimization of the Reaction Conditions}

Table S1. Investigation of NHC catalysts ${ }^{a}$.
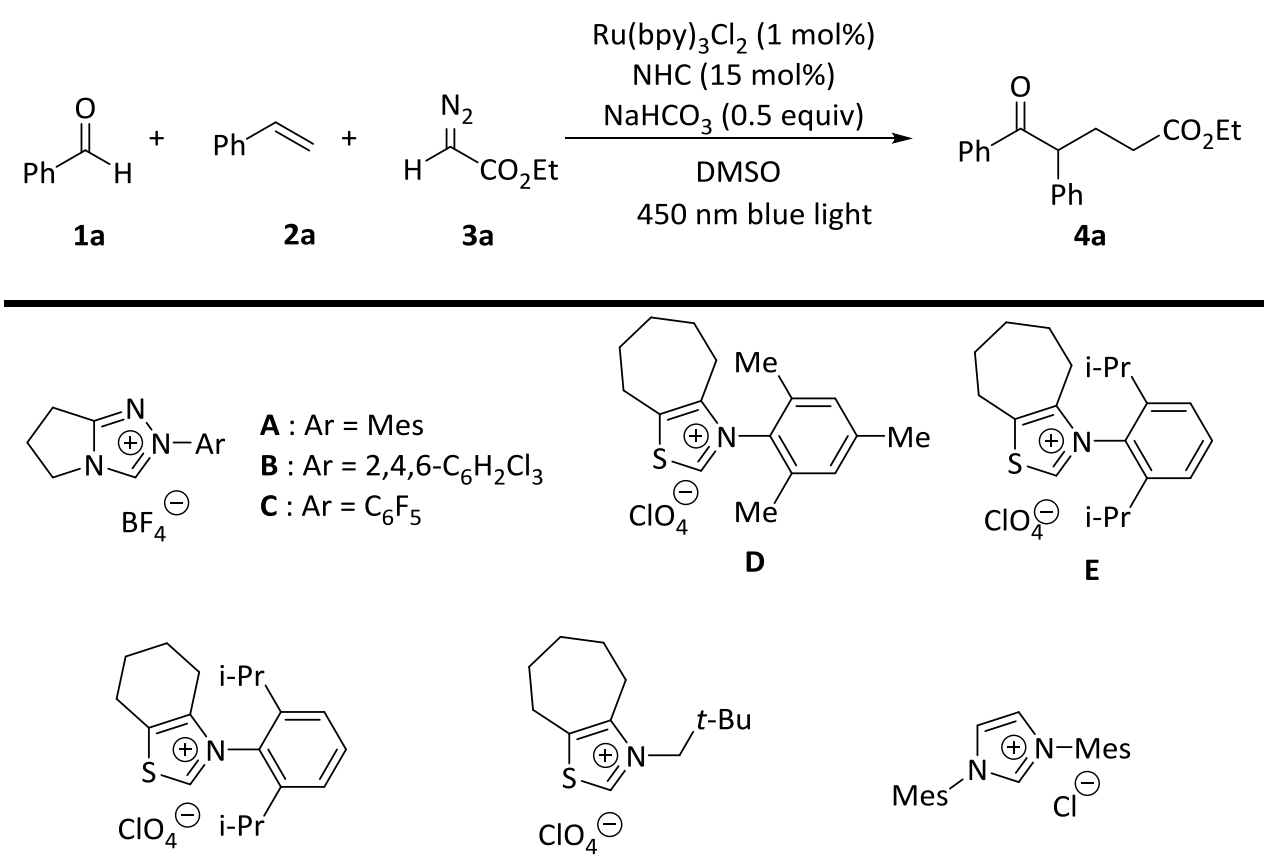

$\mathbf{F}$

G

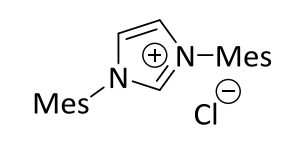

\begin{tabular}{ccc}
\hline $\mathbf{F}$ & $\mathbf{G}$ & $\mathbf{H}$ \\
\hline Entry & NHC & Yield $^{b}(\%)$ \\
\hline 1 & $\mathbf{A}$ & trace \\
2 & $\mathbf{B}$ & trace \\
3 & $\mathbf{C}$ & trace \\
4 & $\mathbf{D}$ & 12 \\
$\mathbf{5}$ & $\mathbf{E}$ & $\mathbf{2 2}$ \\
6 & $\mathbf{F}$ & trace \\
7 & $\mathbf{G}$ & 0 \\
8 & $\mathbf{H}$ & 0 \\
\hline
\end{tabular}

${ }^{a}$ Reaction conditions: 1a $(0.10 \mathrm{mmol}), \mathbf{2 a}(0.10 \mathrm{mmol}), \mathbf{3 a}(0.12 \mathrm{mmol}), \mathrm{NHC}$ cat. $(15$ mol\%), $\mathrm{NaHCO}_{3}(50 \mathrm{~mol} \%), \mathrm{Ru}(\mathrm{bpy})_{3} \mathrm{Cl}_{2}$ (1 mol\%), DMSO (1.0 mL), Ar, room temperature, $450 \mathrm{~nm}$ blue LED, $5 \mathrm{~h} .{ }^{b}$ Isolated yield after column purification. 
Table S2. Investigation of bases ${ }^{a}$.

Ru(bpy) $\mathrm{Cl}_{2}(1 \mathrm{~mol} \%)$
$\mathrm{E}(15 \mathrm{~mol})$
base(0.5 equiv)

${ }^{a}$ Reaction conditions: 1a $(0.10 \mathrm{mmol}), \mathbf{2 a}(0.10 \mathrm{mmol}), \mathbf{3 a}(0.12 \mathrm{mmol})$, cat. E $(15$ mol\%), base (50 mol\%), $\mathrm{Ru}(\text { bpy })_{3} \mathrm{Cl}_{2}(1 \mathrm{~mol} \%)$, DMSO $(1.0 \mathrm{~mL})$, Ar, room temperature, $450 \mathrm{~nm}$ blue LED, $5 \mathrm{~h} .{ }^{b}$ Isolated yield after column purification.

Table S3. Investigation of photocatalysts ${ }^{a}$.

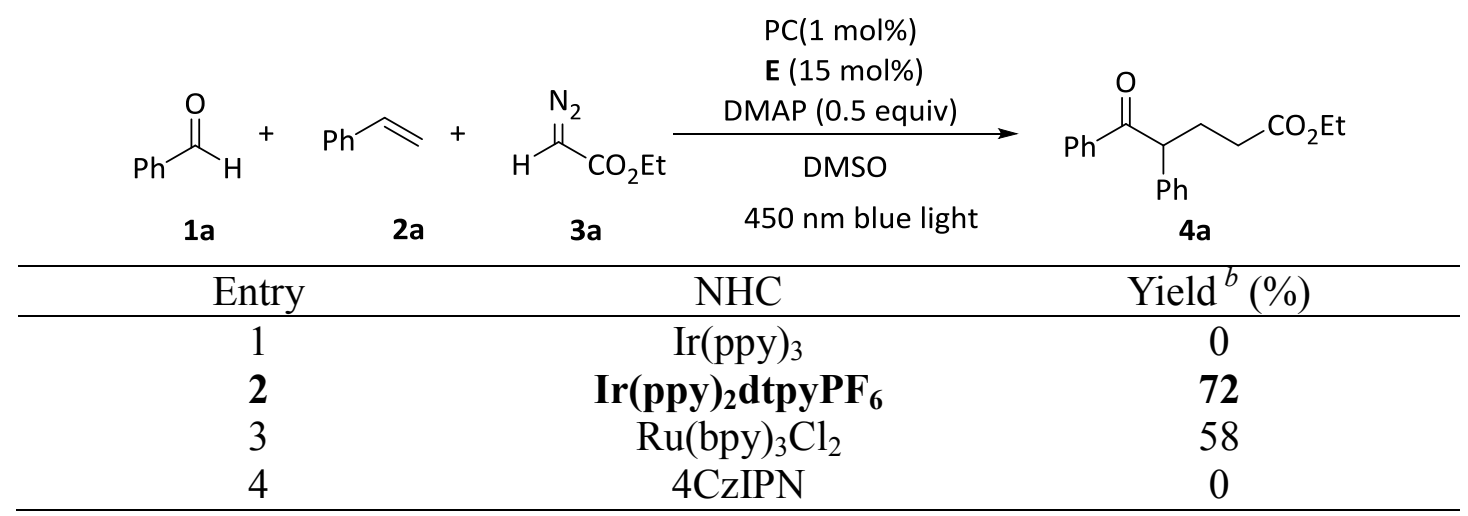

${ }^{a}$ Reaction conditions: 1a $(0.10 \mathrm{mmol}), \mathbf{2 a}(0.10 \mathrm{mmol}), \mathbf{3 a}(0.12 \mathrm{mmol})$, cat. $\boldsymbol{E}(15$ mol\%), DMAP (50 mol\%), photocatalyst (1 mol\%), DMSO $(1.0 \mathrm{~mL})$, Ar, room temperature, $450 \mathrm{~nm}$ blue LED, 5 h. ${ }^{b}$ Isolated yield after column purification.

Table S4. Investigation of light ${ }^{a}$.

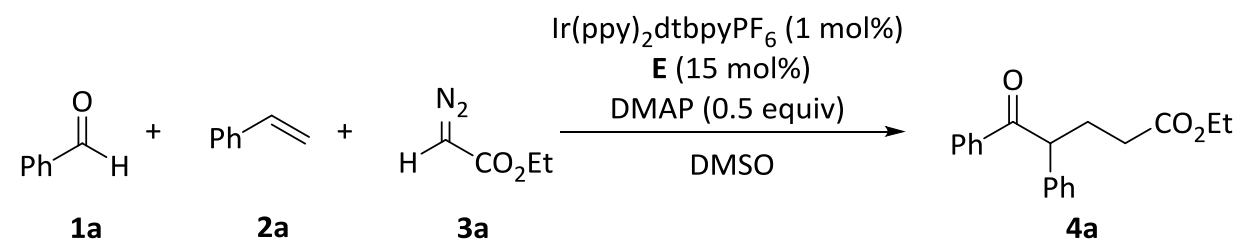




\begin{tabular}{ccc}
\hline Entry & Light & Yield $^{b}(\%)$ \\
\hline 1 & $2 \times 5 \mathrm{~W} \mathrm{CFL}$ & $\operatorname{trace}$ \\
2 & 3W 420 nm blue light (bottom) & 52 \\
3 & 30W 420 nm blue light (side) & 60 \\
4 & 30W 450 nm blue light (side) & 72 \\
5 & 30W 450 nm blue light (side), $60{ }^{\circ} \mathrm{C}$ & trace \\
\hline
\end{tabular}

${ }^{a}$ Reaction conditions: 1a $(0.10 \mathrm{mmol}), \mathbf{2 a}(0.10 \mathrm{mmol})$, 3a $(0.12 \mathrm{mmol})$, cat. $\boldsymbol{E}(15$ mol \%), DMAP (50 mol \%), Ir(ppy) $)_{2} \mathrm{dtbpyPF}_{6}(1 \mathrm{~mol} \%)$, DMSO $(1.0 \mathrm{~mL})$, Ar, room temperature, $5 \mathrm{~h} .{ }^{b}$ Isolated yield after column purification.

Table S5. Investigation of solvent ${ }^{a}$.

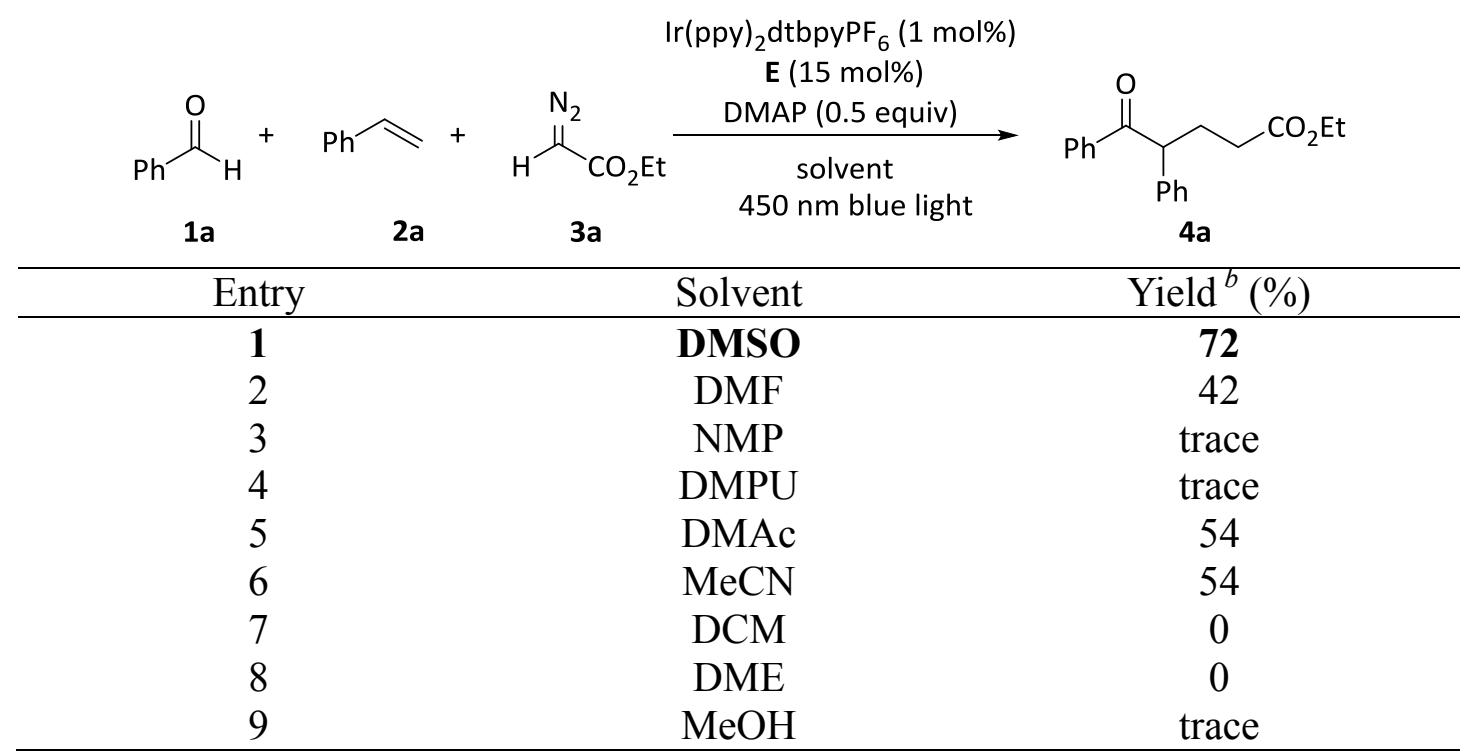

${ }^{a}$ Reaction conditions: 1a $(0.10 \mathrm{mmol}), \mathbf{2 a}(0.10 \mathrm{mmol}), 3 \mathbf{3 a}(0.12 \mathrm{mmol})$, cat. $\boldsymbol{E}(15$ mol\%), DMAP (50 mol\%), $\operatorname{Ir}(\text { ppy })_{2} \mathrm{dtbpyPF}_{6}(1 \mathrm{~mol} \%)$, solvent $(1.0 \mathrm{~mL})$, Ar, room temperature, $450 \mathrm{~nm}$ blue LED, 5 h. ${ }^{b}$ Isolated yield after column purification.

Table S6. Investigation of amounts of reactants, catalysts, and additives ${ }^{a}$.

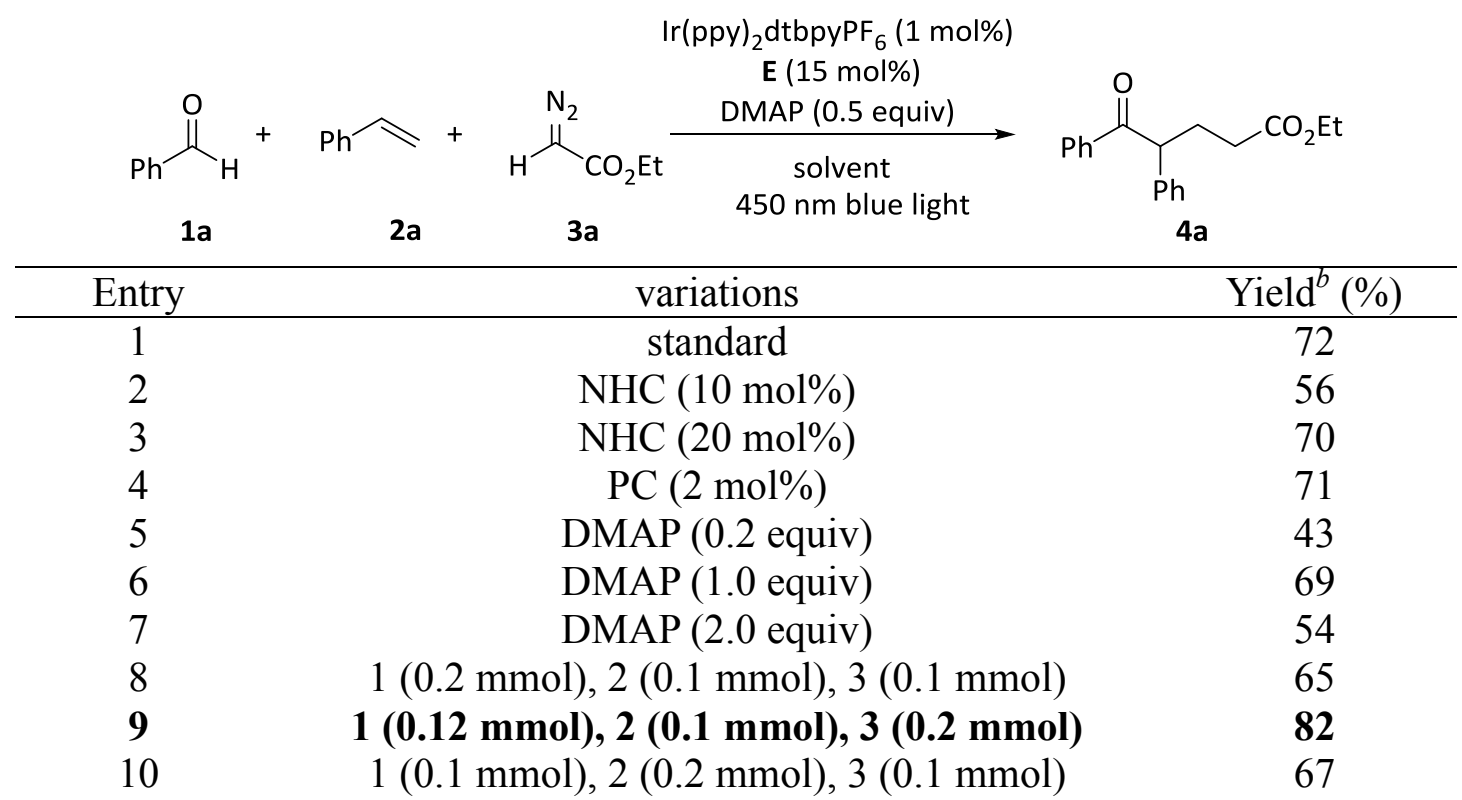


${ }^{a}$ Reaction conditions: 1a $(0.10 \mathrm{mmol}), \mathbf{2 a}(0.10 \mathrm{mmol}), \mathbf{3 a}(0.12 \mathrm{mmol})$, cat. $\boldsymbol{E}(15$ mol \%), DMAP (50 mol \%), Ir(ppy) $)_{2} \mathrm{dtbpyPF}_{6}(1 \mathrm{~mol} \%)$, DMSO (1.0 mL), Ar, room temperature, $450 \mathrm{~nm}$ blue LED, $5 \mathrm{~h} .{ }^{b}$ Isolated yield after column purification.

\section{Scope of other substrates}
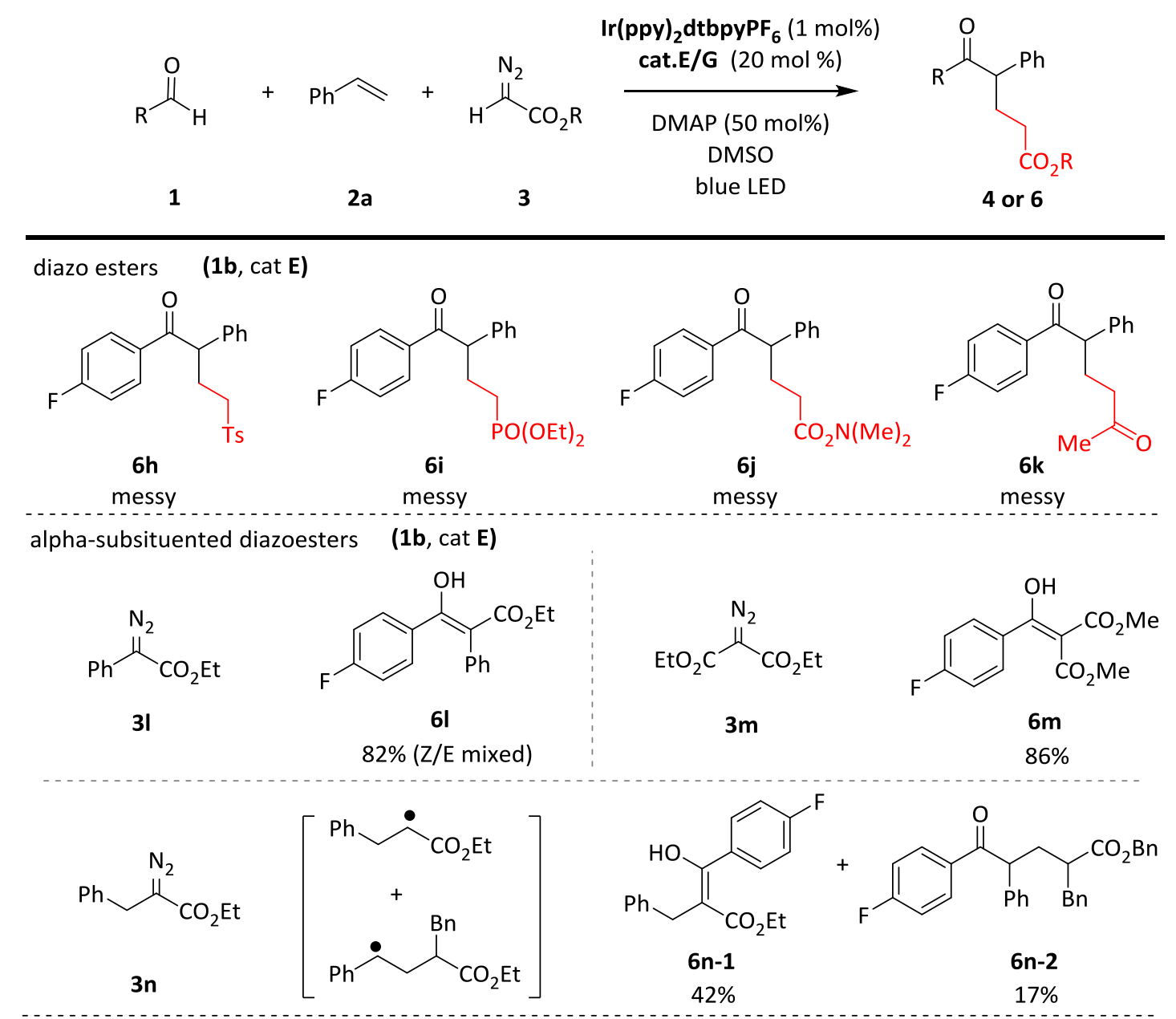

aldehydes<smiles>CCOC(=O)CCC(C(=O)CCc1ccccc1)c1ccccc1</smiles>

$4 r$

cat. E : trace $(80 \% \mathbf{1 r}$ recovered) cat. $\mathbf{G}:$ n.r.<smiles>CCOC(=O)CCC(C(=O)/C=C/c1ccccc1)c1ccccc1</smiles>

4s

cat. $\mathbf{E}:$ n.r. cat. $\mathbf{G}:$ n.r.<smiles>CCOC(=O)CCC(C(=O)C#Cc1ccccc1)c1ccccc1</smiles>

$4 t$

cat. E : n.r. cat. $\mathbf{G}:$ n.r.

Scheme S1. Scope of other substrates.

\section{Substrate Synthesis}

$\mathbf{2} \mathbf{h}^{1}, \mathbf{2 j}^{2}, \mathbf{2} \mathbf{l}^{3}, \mathbf{2 n}-\mathbf{2} \mathbf{p}^{4}$ were known compounds synthesized according to previously reported methods. All the characterization data are consistent with those in the 
reported literature.

$\mathbf{2 k}$ was synthesized from a modified preparation method.

Modified preparation according to the literature ${ }^{3}$ : ACS Catal. 2021, 11, 5166-5171.

To an oven-derived three-necked $50 \mathrm{~mL}$ round-bottom flask charged with a magnetic stir bar, methyltriphenylphosphonium bromide ( 2.0 equiv, $4 \mathrm{mmol}, 1.43 \mathrm{~g}$ ) was added followed by the addition of dry THF $(0.2 \mathrm{M}, 10 \mathrm{~mL})$. The flask was then charged with nitrogen and sealed by septum. The white suspension was stirred in an ice-bath at 0 ${ }^{\circ} \mathrm{C}$. The $t$-BuOK (2.0 equiv, $4 \mathrm{mmol}, 0.45 \mathrm{~g}$ ) was then quickly added after removing the septum under the flow of nitrogen. The resluted yellow suspension was stirred for 30 mins at $0{ }^{\circ} \mathrm{C}$. Followed by the slow addition of 3 -isoquinolinecarboxaldehyde (1.0 equiv, $2 \mathrm{mmol}, 314 \mathrm{mg}$ ), the reaction mixture was warmed up to $\mathrm{rt}$ and was stirred for $3 \mathrm{hrs}$. The reaction was quenched with saturated ammonium chloride solution and was extracted with ethyl acetate for three times. The combined organic layers were dried over $\mathrm{Na}_{2} \mathrm{SO}_{4}$, filtered, concentrated in vacuo, and purified via column chromatography to afford the 3 -ethenyl-isoquinoline as a pure product (124 mg, $40 \%$ ).

${ }^{1} \mathrm{H}$ NMR (400 MHz, $\left.\mathrm{CDCl}_{3}\right) \delta 8.17-8.02(\mathrm{~m}, 2 \mathrm{H}), 7.79(\mathrm{dd}, \mathrm{J}=8.1,1.5 \mathrm{~Hz}, 1 \mathrm{H})$, $7.72(\mathrm{ddd}, \mathrm{J}=8.5,6.9,1.5 \mathrm{~Hz}, 1 \mathrm{H}), 7.62(\mathrm{~d}, \mathrm{~J}=8.6 \mathrm{~Hz}, 1 \mathrm{H}), 7.52(\mathrm{ddd}, \mathrm{J}=8.1,6.9$, $1.2 \mathrm{~Hz}, 1 \mathrm{H}), 7.07$ (dd, J = 17.7, $10.9 \mathrm{~Hz}, 1 \mathrm{H}), 6.30(\mathrm{dd}, \mathrm{J}=17.7,0.9 \mathrm{~Hz}, 1 \mathrm{H}), 5.69$ (dd, J = 10.9, $0.9 \mathrm{~Hz}, 1 \mathrm{H}$ ).

${ }^{13} \mathrm{C}$ NMR $\left(100 \mathrm{MHz}, \mathrm{CDCl}_{3}\right) \delta 156.08,147.97,137.97,136.42,129.71,129.36$, 127.50, 126.37, 119.95, 118.38.

HRMS-ESI $(\mathrm{m} / \mathrm{z})[\mathrm{M}+\mathrm{H}]^{+}$calc'd for $\mathrm{C}_{11} \mathrm{H}_{10} \mathrm{~N}, 156.0808$, found 156.0815.

Compound 2k ( $\left.{ }^{1} \mathrm{H} \mathrm{NMR,} \mathrm{CDCl}_{3}, 400 \mathrm{MHz}\right)$

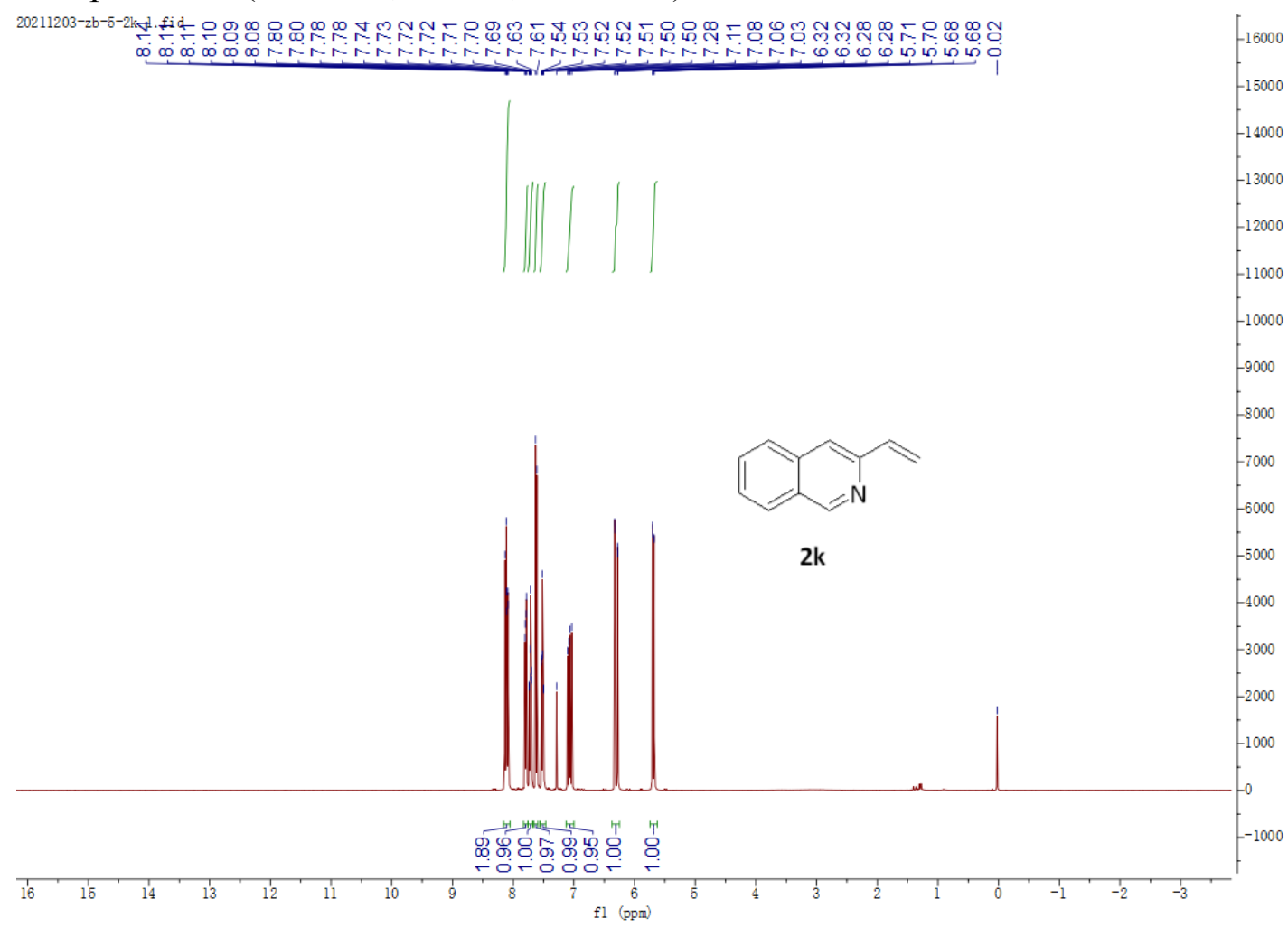


Compound 2k $\left({ }^{13} \mathrm{C} \mathrm{NMR}, \mathrm{CDCl}_{3}, 100 \mathrm{MHz}\right)$

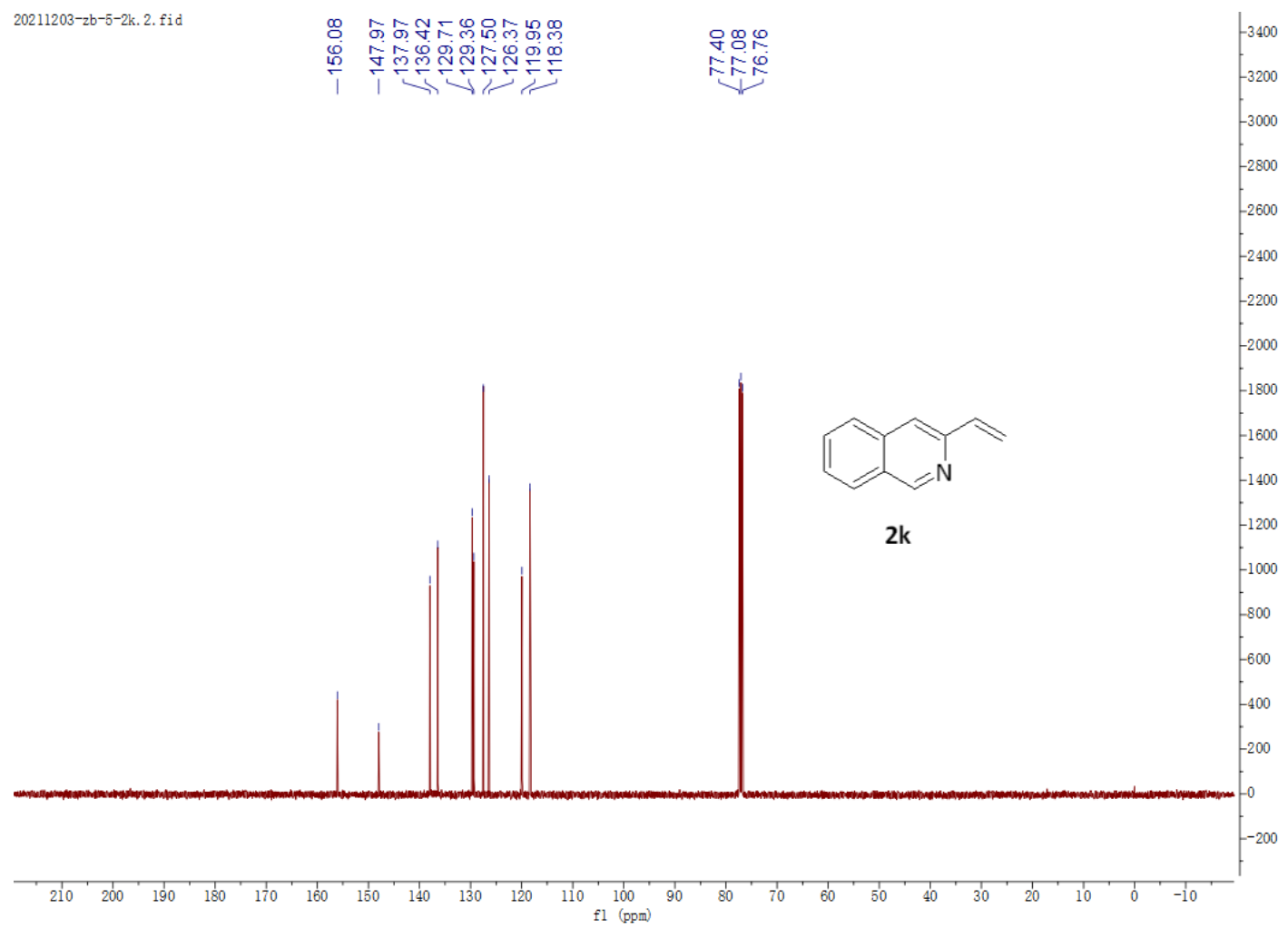

$\mathbf{3 b}^{5}, \mathbf{3 d}-\mathbf{3} \mathbf{e}^{5}, \mathbf{3 f}^{6}, \mathbf{3} \mathbf{g}^{7}$ were known compounds and synthesized by published methods.

\section{Characterization}

ethyl 5-oxo-4,5-diphenylpentanoate (4a)<smiles>CCOC(=O)CCC(C(=O)c1ccccc1)c1ccccc1</smiles>

4a

$\mathrm{PE} / \mathrm{EA}=30 / 1$. Colorless oil, 48.5mg, $82 \%$.

${ }^{1} \mathrm{H}$ NMR (400 MHz, $\left.\mathrm{CDCl}_{3}\right) \delta 7.98(\mathrm{~d}, J=7.7 \mathrm{~Hz}, 2 \mathrm{H}), 7.50(\mathrm{t}, J=7.1 \mathrm{~Hz}, 1 \mathrm{H}), 7.40$ $(\mathrm{t}, J=7.4 \mathrm{~Hz}, 2 \mathrm{H}), 7.31(\mathrm{~s}, 4 \mathrm{H}), 7.27-7.18(\mathrm{~m}, 1 \mathrm{H}), 4.70(\mathrm{t}, J=7.3 \mathrm{~Hz}, 1 \mathrm{H}), 4.13(\mathrm{q}$, $J=7.0 \mathrm{~Hz}, 2 \mathrm{H}), 2.52-2.44(\mathrm{~m}, 1 \mathrm{H}), 2.32(\mathrm{t}, J=7.0 \mathrm{~Hz}, 2 \mathrm{H}), 2.24-2.15(\mathrm{~m}, 1 \mathrm{H})$, $1.25(\mathrm{t}, J=7.1 \mathrm{~Hz}, 3 \mathrm{H})$.

${ }^{13} \mathrm{C}$ NMR $\left(100 \mathrm{MHz}, \mathrm{CDCl}_{3}\right) \delta 199.3,173.3,138.8,136.6,132.9,129.1,128.7,128.5$, $128.3,127.3,60.4,52.4,31.9,28.8,14.2$.

HRMS-ESI $(\mathrm{m} / \mathrm{z})[\mathrm{M}+\mathrm{H}]^{+}$calc'd for $\mathrm{C}_{19} \mathrm{H}_{21} \mathrm{O}_{3}, 297.1485$, found 297.1489.

ethyl 5-(4-fluorophenyl)-5-oxo-4-phenylpentanoate (4b) 
<smiles>CCOC(=O)CCC(C(=O)c1ccc(F)cc1)c1ccccc1</smiles>

4b

$\mathrm{PE} / \mathrm{EA}=30 / 1$. Colorless oil, $57.7 \mathrm{mg}, 92 \%$

${ }^{1} \mathrm{H}$ NMR (400 MHz, $\left.\mathrm{CDCl}_{3}\right) \delta 8.04-7.97(\mathrm{~m}, 2 \mathrm{H}), 7.36-7.22(\mathrm{~m}, 5 \mathrm{H}), 7.06(\mathrm{t}, J=$ $8.1 \mathrm{~Hz}, 2 \mathrm{H}), 4.65(\mathrm{t}, J=7.2 \mathrm{~Hz}, 1 \mathrm{H}), 4.13(\mathrm{q}, J=7.1 \mathrm{~Hz}, 2 \mathrm{H}), 2.49-2.42(\mathrm{~m}, 1 \mathrm{H})$, $2.31(\mathrm{t}, J=7.0 \mathrm{~Hz}, 2 \mathrm{H}), 2.22-2.15(\mathrm{~m}, 1 \mathrm{H}), 1.25(\mathrm{t}, J=7.1 \mathrm{~Hz}, 3 \mathrm{H})$.

${ }^{19} \mathrm{~F} \mathrm{NMR}\left(376 \mathrm{MHz}, \mathrm{CDCl}_{3}\right) \delta-105.31(\mathrm{~s})$.

${ }^{13} \mathrm{C}$ NMR $\left(100 \mathrm{MHz}, \mathrm{CDCl}_{3}\right) \delta 197.7,173.3,165.55(\mathrm{~d}, J=254.9 \mathrm{~Hz}), 138.7,133.0$ (d, $J=3.3 \mathrm{~Hz}), 131.4(\mathrm{~d}, J=9.3 \mathrm{~Hz}), 129.1,128.3,127.4,115.6(\mathrm{~d}, J=21.8 \mathrm{~Hz})$, 60.4, 52.4, 31.7, 28.7, 14.2.

HRMS-ESI $(\mathrm{m} / \mathrm{z})[\mathrm{M}+\mathrm{H}]^{+}$calc'd for $\mathrm{C}_{19} \mathrm{H}_{20} \mathrm{FO}_{3}, 315.1391$, found 315.1396 .

ethyl 5-oxo-4-phenyl-5-(p-tolyl)pentanoate (4c)<smiles>CCOC(=O)CCC(C(=O)c1ccc(C)cc1)c1ccccc1</smiles>

$\mathrm{PE} / \mathrm{EA}=30 / 1$. Colorless oil, $48.2 \mathrm{mg}, 78 \%$

${ }^{1} \mathrm{H}$ NMR $\left(400 \mathrm{MHz}, \mathrm{CDCl}_{3}\right) \delta 7.88(\mathrm{~d}, J=7.7 \mathrm{~Hz}, 2 \mathrm{H}), 7.31(\mathrm{~d}, J=4.0 \mathrm{~Hz}, 4 \mathrm{H}), 7.26$ $-7.17(\mathrm{~m}, 3 \mathrm{H}), 4.67(\mathrm{t}, J=7.3 \mathrm{~Hz}, 1 \mathrm{H}), 4.13(\mathrm{q}, J=7.1 \mathrm{~Hz}, 2 \mathrm{H}), 2.50-2.44(\mathrm{~m}, 1 \mathrm{H})$, $2.37(\mathrm{~s}, 3 \mathrm{H}), 2.34-2.28(\mathrm{~m}, 2 \mathrm{H}), 2.22-2.16(\mathrm{~m}, 1 \mathrm{H}), 1.25(\mathrm{t}, J=7.1 \mathrm{~Hz}, 3 \mathrm{H})$.

${ }^{13} \mathrm{C}$ NMR $\left(100 \mathrm{MHz}, \mathrm{CDCl}_{3}\right) \delta 198.9,173.3,143.8,139.1,134.1,129.2,129.0,128.9$, $128.3,127.2,60.3,52.3,31.9,28.8,21.6,14.2$.

HRMS-ESI $(\mathrm{m} / \mathrm{z})[\mathrm{M}+\mathrm{H}]^{+}$calc'd for $\mathrm{C}_{20} \mathrm{H}_{23} \mathrm{O}_{3}, 311.1642$, found 311.1650 .

ethyl 5-(3-methoxyphenyl)-5-oxo-4-phenylpentanoate (4d)<smiles>CCOC(=O)CCC(C(=O)c1ccccc1)c1ccccc1</smiles>

$\mathrm{PE} / \mathrm{EA}=30 / 1$. Colorless oil, $56.5 \mathrm{mg}, 87 \%$

${ }^{1} \mathrm{H}$ NMR $\left(400 \mathrm{MHz}, \mathrm{CDCl}_{3}\right) \delta 7.56(\mathrm{~d}, J=7.6 \mathrm{~Hz}, 1 \mathrm{H}), 7.50(\mathrm{~s}, 1 \mathrm{H}), 7.34-7.28(\mathrm{~m}$, $5 \mathrm{H}), 7.27-7.17(\mathrm{~m}, 1 \mathrm{H}), 7.05(\mathrm{~d}, J=8.0 \mathrm{~Hz}, 1 \mathrm{H}), 4.67(\mathrm{t}, J=7.2 \mathrm{~Hz}, 1 \mathrm{H}), 4.13(\mathrm{~d}, J$ $=7.1 \mathrm{~Hz}, 2 \mathrm{H}), 3.82(\mathrm{~s}, 3 \mathrm{H}), 2.52-2.43(\mathrm{~m}, 1 \mathrm{H}), 2.31(\mathrm{t}, J=7.0 \mathrm{~Hz}, 2 \mathrm{H}), 2.23-2.14$ $(\mathrm{m}, 1 \mathrm{H}), 1.25(\mathrm{t}, J=7.1 \mathrm{~Hz}, 3 \mathrm{H})$.

${ }^{13} \mathrm{C}$ NMR $\left(100 \mathrm{MHz}, \mathrm{CDCl}_{3}\right) \delta 199.1,173.3,159.7,138.9,138.0,129.5,129.0,128.3$, $127.3,121.4,119.5,113.0,60.4,55.4,52.6,31.9,28.8,14.2$. 
HRMS-ESI (m/z) $[\mathrm{M}+\mathrm{H}]^{+}$calc'd for $\mathrm{C}_{20} \mathrm{H}_{23} \mathrm{O}_{4}, 327.1591$, found 327.1588.

ethyl 5-oxo-4-phenyl-5-(3-(trifluoromethyl)phenyl)pentanoate (4e)<smiles>CCOC(=O)CCC(C(=O)c1cccc(C(F)(F)F)c1)c1ccccc1</smiles>

$\mathrm{PE} / \mathrm{EA}=20 / 1$. Colorless oil, $60.6 \mathrm{mg}, 83 \%$

${ }^{1} \mathrm{H}$ NMR (400 MHz, $\left.\mathrm{CDCl}_{3}\right) \delta 8.24(\mathrm{~s}, 1 \mathrm{H}), 8.13(\mathrm{~d}, J=7.9 \mathrm{~Hz}, 1 \mathrm{H}), 7.75(\mathrm{~d}, J=7.8$ $\mathrm{Hz}, 1 \mathrm{H}), 7.53$ (t, $J=7.8 \mathrm{~Hz}, 1 \mathrm{H}), 7.41-7.22(\mathrm{~m}, 5 \mathrm{H}), 4.69$ (t, $J=7.3 \mathrm{~Hz}, 1 \mathrm{H}), 4.14$ $(\mathrm{q}, J=7.2 \mathrm{~Hz}, 2 \mathrm{H}), 2.51-2.44(\mathrm{~m}, 1 \mathrm{H}), 2.32(\mathrm{t}, J=7.1 \mathrm{~Hz}, 2 \mathrm{H}), 2.25-2.18(\mathrm{~m}, 1 \mathrm{H})$, $1.26(\mathrm{~d}, J=7.2 \mathrm{~Hz}, 3 \mathrm{H})$.

${ }^{19} \mathrm{~F}$ NMR $\left(376 \mathrm{MHz}, \mathrm{CDCl}_{3}\right) \delta-62.91$.

${ }^{13} \mathrm{C}$ NMR $\left(100 \mathrm{MHz}, \mathrm{CDCl}_{3}\right) \delta 197.9,173.2,138.1,137.0,131.2(\mathrm{~d}, \mathrm{~J}=33.0 \mathrm{~Hz})$, 131.0, 129.3, 129.2, 128.3, 127.6, 125.6 (d, J = 3.9 Hz), 123.6 (d, J = 272.2 Hz), 60.5, 52.7, 31.6, 28.6, 14.2.

HRMS-ESI (m/z) $[\mathrm{M}+\mathrm{H}]^{+}$calc'd for $\mathrm{C}_{20} \mathrm{H}_{20} \mathrm{~F}_{3} \mathrm{O}_{3}, 365.1359$, found 365.1351 .

ethyl 5-(2-hydroxy-5-methylphenyl)-5-oxo-4-phenylpentanoate (4f)<smiles>CCOC(=O)CCC(C(=O)c1cc(C)ccc1O)c1ccccc1</smiles>

$\mathrm{PE} / \mathrm{EA}=20 / 1$. Colorless oil, $47.4 \mathrm{mg}, 73 \%$

${ }^{1} \mathrm{H}$ NMR $\left(400 \mathrm{MHz}, \mathrm{CDCl}_{3}\right) \delta 12.23(\mathrm{~s}, 1 \mathrm{H}), 7.61(\mathrm{~d}, J=2.1 \mathrm{~Hz}, 1 \mathrm{H}), 7.38-7.32(\mathrm{~m}$, $4 \mathrm{H}), 7.29-7.21(\mathrm{~m}, 2 \mathrm{H}), 6.87(\mathrm{~d}, J=8.4 \mathrm{~Hz}, 1 \mathrm{H}), 4.74(\mathrm{t}, J=7.3 \mathrm{~Hz}, 1 \mathrm{H}), 4.14$ (q, $J$ $=7.2 \mathrm{~Hz}, 2 \mathrm{H}), 2.51-2.42(\mathrm{~m}, 1 \mathrm{H}), 2.35-2.29(\mathrm{~m}, 2 \mathrm{H}), 2.26(\mathrm{~s}, 3 \mathrm{H}), 2.21-2.16(\mathrm{~m}$, $1 \mathrm{H}), 1.26(\mathrm{t}, J=7.1 \mathrm{~Hz}, 3 \mathrm{H})$.

${ }^{13} \mathrm{C} \mathrm{NMR}\left(100 \mathrm{MHz}, \mathrm{CDCl}_{3}\right) \delta 205.3,173.2,161.2,138.7,137.5,130.1,129.2,128.1$, $127.9,127.5,118.6,118.4,60.5,51.6,31.8,28.6,20.7,14.2$.

HRMS-ESI (m/z) $[\mathrm{M}+\mathrm{H}]^{+}$calc'd for $\mathrm{C}_{20} \mathrm{H}_{23} \mathrm{O}_{4}, 327.1591$, found 327.1596.

ethyl 5-(2-hydroxy-5-methylphenyl)-5-oxo-4-phenylpentanoate (4g)<smiles>CCOC(=O)CCC(C(=O)c1ccc2c(c1)OCCO2)c1ccccc1</smiles>

$4 \mathrm{~g}$ 
$\mathrm{PE} / \mathrm{EA}=30 / 1$. Colorless oil, $60.4 \mathrm{mg}, 85 \%$

${ }^{1} \mathrm{H}$ NMR $\left(400 \mathrm{MHz}, \mathrm{CDCl}_{3}\right) \delta 7.55-7.49(\mathrm{~m}, 2 \mathrm{H}), 7.33-7.27(\mathrm{~m}, 4 \mathrm{H}), 7.23(\mathrm{ddd}, J$ $=9.6,8.1,3.9 \mathrm{~Hz}, 1 \mathrm{H}), 6.84(\mathrm{~d}, J=9.1 \mathrm{~Hz}, 1 \mathrm{H}), 4.60(\mathrm{t}, J=7.3 \mathrm{~Hz}, 1 \mathrm{H}), 4.34-4.20$ $(\mathrm{m}, 4 \mathrm{H}), 4.12(\mathrm{q}, J=7.1 \mathrm{~Hz}, 2 \mathrm{H}), 2.51-2.40(\mathrm{~m}, 1 \mathrm{H}), 2.33-2.25(\mathrm{~m}, 2 \mathrm{H}), 2.20-$ $2.13(\mathrm{~m}, 1 \mathrm{H}), 1.25(\mathrm{t}, J=7.1 \mathrm{~Hz}, 3 \mathrm{H})$.

${ }^{13} \mathrm{C} \mathrm{NMR}\left(100 \mathrm{MHz}, \mathrm{CDCl}_{3}\right) \delta 197.7,173.3,147.9,143.2,139.2,130.4,129.0,128.2$, 127.2, 122.9, 118.3, 117.2, 64.7, 64.1, 60.4, 52.1, 31.9, 28.9, 14.3.

HRMS-ESI $(\mathrm{m} / \mathrm{z})[\mathrm{M}+\mathrm{H}]^{+}$calc'd for $\mathrm{C}_{21} \mathrm{H}_{23} \mathrm{O}_{5}, 355.1540$, found 355.1544 .

ethyl 5-(naphthalen-2-yl)-5-oxo-4-phenylpentanoate (4h)<smiles>CCOC(=O)CCC(C(=O)c1ccc2ccccc2c1)c1ccccc1</smiles>

$4 \mathrm{~h}$

$\mathrm{PE} / \mathrm{EA}=30 / 1$. Colorless oil, $59.5 \mathrm{mg}, 86 \%$

${ }^{1} \mathrm{H}$ NMR $\left(400 \mathrm{MHz}, \mathrm{CDCl}_{3}\right) \delta 8.51(\mathrm{~s}, 1 \mathrm{H}), 8.03(\mathrm{~d}, J=8.6 \mathrm{~Hz}, 1 \mathrm{H}), 7.93(\mathrm{~d}, J=8.0$ $\mathrm{Hz}, 1 \mathrm{H}), 7.84(\mathrm{~d}, J=8.5 \mathrm{~Hz}, 2 \mathrm{H}), 7.62-7.47$ (m, 2H), 7.38 (d, $J=7.8 \mathrm{~Hz}, 2 \mathrm{H}), 7.32$ $(\mathrm{t}, J=7.4 \mathrm{~Hz}, 2 \mathrm{H}), 7.27-7.13(\mathrm{~m}, 1 \mathrm{H}), 4.87(\mathrm{t}, J=7.2 \mathrm{~Hz}, 1 \mathrm{H}), 4.14(\mathrm{q}, J=7.2 \mathrm{~Hz}$, $2 \mathrm{H}), 2.57-2.50(\mathrm{~m}, 1 \mathrm{H}), 2.36(\mathrm{t}, J=7.1 \mathrm{~Hz}, 2 \mathrm{H}), 2.29-2.22(\mathrm{~m}, 1 \mathrm{H}), 1.26(\mathrm{t}, J=7.2$ $\mathrm{Hz}, 3 \mathrm{H})$.

${ }^{13} \mathrm{C}$ NMR $\left(100 \mathrm{MHz}, \mathrm{CDCl}_{3}\right) \delta 199.3,173.3,139.0,135.5,133.9,132.5,130.5,129.7$, 129.1, 128.5, 128.4, 128.3, 127.7, 127.3, 126.7, 124.5, 60.4, 52.5, 31.9, 28.9, 14.2 . HRMS-ESI $(\mathrm{m} / \mathrm{z})[\mathrm{M}+\mathrm{H}]^{+}$calc'd for $\mathrm{C}_{23} \mathrm{H}_{23} \mathrm{O}_{3}, 347.1642$, found 347.1648.

ethyl 5-oxo-4-phenyl-5-(pyridin-3-yl)pentanoate (4i)<smiles>CCOC(=O)CCC(C(=O)c1cccnc1)c1ccccc1</smiles>

$4 \mathbf{i}$

$\mathrm{PE} / \mathrm{EA}=10 / 1$ to $5 / 1$. Colorless oil, $45.0 \mathrm{mg}, 76 \%$

${ }^{1} \mathrm{H}$ NMR (400 MHz, $\left.\mathrm{CDCl}_{3}\right) \delta 9.17(\mathrm{~s}, 1 \mathrm{H}), 8.70(\mathrm{~d}, J=3.5 \mathrm{~Hz}, 1 \mathrm{H}), 8.23(\mathrm{~d}, J=8.0$ $\mathrm{Hz}, 1 \mathrm{H}), 7.43-7.17(\mathrm{~m}, 6 \mathrm{H}), 4.65(\mathrm{t}, J=7.3 \mathrm{~Hz}, 1 \mathrm{H}), 4.13$ (q, $J=7.0 \mathrm{~Hz}, 2 \mathrm{H}), 2.52-$ $2.44(\mathrm{~m}, 1 \mathrm{H}), 2.31(\mathrm{t}, J=7.0 \mathrm{~Hz}, 2 \mathrm{H}), 2.22-2.15(\mathrm{~m}, 1 \mathrm{H}), 1.25(\mathrm{t}, J=7.1 \mathrm{~Hz}, 3 \mathrm{H})$.

${ }^{13} \mathrm{C} \mathrm{NMR}\left(100 \mathrm{MHz}, \mathrm{CDCl}_{3}\right) \delta 198.1,173.2,153.1,150.1,137.9,136.1,131.8,129.3$, 128.4, 127.7, 123.6, 60.5, 53.0, 31.6, 28.4, 14.2.

HRMS-ESI $(\mathrm{m} / \mathrm{z})[\mathrm{M}+\mathrm{H}]^{+}$calc'd for $\mathrm{C}_{18} \mathrm{H}_{20} \mathrm{NO}_{3}, 298.1438$, found 298.1443.

ethyl 5-oxo-4-phenyl-5-(pyridin-2-yl)pentanoate (4j) 
$\underbrace{\mathrm{O}}_{\mathrm{N}=\mathrm{Nh}} \mathrm{CO}_{2} \mathrm{Et}$

$4 \mathbf{j}$

$\mathrm{PE} / \mathrm{EA}=10 / 1$ to $5 / 1$. Colorless oil, $37.5 \mathrm{mg}, 63 \%$

${ }^{1} \mathrm{H}$ NMR $\left(400 \mathrm{MHz}, \mathrm{CDCl}_{3}\right) \delta 8.66(\mathrm{~d}, J=4.5 \mathrm{~Hz}, 1 \mathrm{H}), 8.02(\mathrm{~d}, J=7.8 \mathrm{~Hz}, 1 \mathrm{H}), 7.79$ $-7.75(\mathrm{~m}, 1 \mathrm{H}), 7.43-7.36(\mathrm{~m}, 3 \mathrm{H}), 7.34-7.22(\mathrm{~m}, 2 \mathrm{H}), 7.19$ (t, $J=7.3 \mathrm{~Hz}, 1 \mathrm{H})$, $5.44(\mathrm{t}, J=7.3 \mathrm{~Hz}, 1 \mathrm{H}), 4.11(\mathrm{q}, J=7.1 \mathrm{~Hz}, 2 \mathrm{H}), 2.54-2.41(\mathrm{~m}, 1 \mathrm{H}), 2.40-2.20(\mathrm{~m}$, $3 \mathrm{H}), 1.24(\mathrm{t}, J=7.1 \mathrm{~Hz}, 3 \mathrm{H})$.

${ }^{13} \mathrm{C}$ NMR $\left(100 \mathrm{MHz}, \mathrm{CDCl}_{3}\right) \delta 200.7,173.3,152.8,148.9,138.4,136.8,129.0,128.6$, $127.0,122.7,60.3,49.9,32.3,27.9,14.2$.

HRMS-ESI $(\mathrm{m} / \mathrm{z})[\mathrm{M}+\mathrm{H}]^{+}$calc'd for $\mathrm{C}_{18} \mathrm{H}_{20} \mathrm{NO}_{3}, 298.1438$, found 298.1441 .

ethyl 5-oxo-4-phenyl-5-(1-tosyl-1H-pyrrol-3-yl)pentanoate (4k)<smiles>CCOC(=O)CCC(C(=O)c1ccn(S)c1)c1ccccc1</smiles>

$\mathrm{PE} / \mathrm{EA}=10 / 1$ to $5 / 1$. Colorless oil, $63.0 \mathrm{mg}, 72 \%$

${ }^{1} \mathrm{H}$ NMR $\left(400 \mathrm{MHz}, \mathrm{CDCl}_{3}\right) \delta 7.76-7.62(\mathrm{~m}, 3 \mathrm{H}), 7.36-7.23(\mathrm{~m}, 7 \mathrm{H}), 7.07-7.00$ $(\mathrm{m}, 1 \mathrm{H}), 6.67-6.61(\mathrm{~m}, 1 \mathrm{H}), 4.27(\mathrm{~d}, J=7.4 \mathrm{~Hz}, 1 \mathrm{H}), 4.12(\mathrm{q}, J=6.9 \mathrm{~Hz}, 2 \mathrm{H}), 2.43$ $(\mathrm{s}, 3 \mathrm{H}), 2.43-2.35(\mathrm{~m}, 1 \mathrm{H}), 2.26(\mathrm{t}, J=7.1 \mathrm{~Hz}, 2 \mathrm{H}), 2.22-2.02(\mathrm{~m}, 1 \mathrm{H}), 1.25(\mathrm{t}, J=$ $7.1 \mathrm{~Hz}, 3 \mathrm{H})$.

${ }^{13} \mathrm{C} \mathrm{NMR}\left(100 \mathrm{MHz}, \mathrm{CDCl}_{3}\right) \delta 194.4,173.2,145.9,138.9,135.0,130.3,129.0,128.4$, 128.3, 127.3, 127.2, 125.0, 121.3, 112.9, 60.4, 54.3, 31.7, 28.2, 21.7, 14.2.

HRMS-ESI $(\mathrm{m} / \mathrm{z})[\mathrm{M}+\mathrm{H}]^{+}$calc'd for $\mathrm{C}_{24} \mathrm{H}_{26} \mathrm{NO}_{5} \mathrm{~S}, 440.1526$, found 440.1530 .

ethyl 5-(furan-3-yl)-5-oxo-4-phenylpentanoate (4l)<smiles>CCOC(=O)CCC(C(=O)c1ccoc1)c1ccccc1</smiles>

41

$\mathrm{PE} / \mathrm{EA}=20 / 1$. Colorless oil, $40.0 \mathrm{mg}, 70 \%$

${ }^{1} \mathrm{H}$ NMR $\left(400 \mathrm{MHz}, \mathrm{CDCl}_{3}\right) \delta 7.96(\mathrm{~s}, 1 \mathrm{H}), 7.39-7.21(\mathrm{~m}, 6 \mathrm{H}), 6.74(\mathrm{~s}, 1 \mathrm{H}), 4.24(\mathrm{t}$, $J=7.3 \mathrm{~Hz}, 1 \mathrm{H}), 4.13(\mathrm{q}, J=7.1 \mathrm{~Hz}, 2 \mathrm{H}), 2.50-2.41(\mathrm{~m}, 1 \mathrm{H}), 2.29$ (t, $J=7.0 \mathrm{~Hz}$, $2 \mathrm{H}), 2.18-2.09(\mathrm{~m}, 1 \mathrm{H}), 1.25(\mathrm{t}, J=7.1 \mathrm{~Hz}, 3 \mathrm{H})$.

${ }^{13} \mathrm{C}$ NMR $\left(100 \mathrm{MHz}, \mathrm{CDCl}_{3}\right) \delta 194.2,173.3,147.8,143.9,138.8,129.1,128.2,127.5$, $127.3,109.1,60.4,55.1,31.7,28.2,14.2$. 
HRMS-ESI $(\mathrm{m} / \mathrm{z})[\mathrm{M}+\mathrm{H}]^{+}$calc'd for $\mathrm{C}_{17} \mathrm{H}_{19} \mathrm{O}_{4}, 287.1278$, found 287.1283.

ethyl 5-oxo-4-phenyl-5-(thiophen-3-yl)pentanoate (4m)

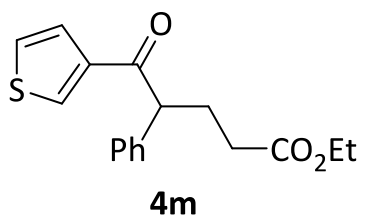

$\mathrm{PE} / \mathrm{EA}=20 / 1$. Colorless oil, 39.0mg, $65 \%$

${ }^{1} \mathrm{H}$ NMR $\left(400 \mathrm{MHz}, \mathrm{CDCl}_{3}\right) \delta 8.06(\mathrm{~d}, J=1.8 \mathrm{~Hz}, 1 \mathrm{H}), 7.54(\mathrm{~d}, J=5.1 \mathrm{~Hz}, 1 \mathrm{H}), 7.37$ $-7.29(\mathrm{~m}, 4 \mathrm{H}), 7.28-7.20(\mathrm{~m}, 2 \mathrm{H}), 4.49$ (t, $J=7.4 \mathrm{~Hz}, 1 \mathrm{H}), 4.13$ (q, $J=7.1 \mathrm{~Hz}, 2 \mathrm{H})$, $2.51-2.42(\mathrm{~m}, 1 \mathrm{H}), 2.31(\mathrm{t}, J=7.1 \mathrm{~Hz}, 2 \mathrm{H}), 2.20-2.09(\mathrm{~m}, 1 \mathrm{H}), 1.25(\mathrm{t}, J=7.1 \mathrm{~Hz}$, $3 \mathrm{H})$.

${ }^{13} \mathrm{C} \mathrm{NMR}\left(100 \mathrm{MHz}, \mathrm{CDCl}_{3}\right) \delta 193.6,173.3,141.8,138.9,132.9,129.1,128.3,127.5$, $127.39,126.1,60.4,54.2,31.8,28.5,14.2$.

HRMS-ESI $(\mathrm{m} / \mathrm{z})[\mathrm{M}+\mathrm{H}]^{+}$calc'd for $\mathrm{C}_{17} \mathrm{H}_{19} \mathrm{O}_{3} \mathrm{~S}, 303.1049$, found 303.1055.

ethyl 5-oxo-4-phenyl-5-(1H-pyrazol-3-yl)pentanoate (4n)<smiles>CCOC(=O)CCC(C(=O)c1cc[nH]n1)c1ccccc1</smiles>

$4 n$

$\mathrm{PE} / \mathrm{EA}=3 / 1$. Colorless oil, 38.2mg, $67 \%$

${ }^{1} \mathrm{H}$ NMR $\left(400 \mathrm{MHz}, \mathrm{CDCl}_{3}\right) \delta 7.61(\mathrm{~d}, J=2.3 \mathrm{~Hz}, 1 \mathrm{H}), 7.39-7.32(\mathrm{~m}, 3 \mathrm{H}), 7.32-$ $7.29(\mathrm{~m}, 2 \mathrm{H}), 7.27-7.20(\mathrm{~m}, 1 \mathrm{H}), 6.81(\mathrm{~s}, 1 \mathrm{H}), 4.73(\mathrm{t}, J=6.0 \mathrm{~Hz}, 1 \mathrm{H}), 4.12(\mathrm{q}, J=$ $7.1 \mathrm{~Hz}, 2 \mathrm{H}), 2.56-2.41(\mathrm{~m}, 1 \mathrm{H}), 2.36-2.29(\mathrm{~m}, 2 \mathrm{H}), 2.29-2.14(\mathrm{~m}, 1 \mathrm{H}), 1.24(\mathrm{t}, J$ $=7.1 \mathrm{~Hz}, 3 \mathrm{H})$.

${ }^{13} \mathrm{C}$ NMR $\left(100 \mathrm{MHz}, \mathrm{CDCl}_{3}\right) \delta 173.3,138.4,128.9,128.5,127.4,77.4,77.1,76.7$, $60.5,53.3,31.9,27.9,14.2$.

HRMS-ESI $(\mathrm{m} / \mathrm{z})[\mathrm{M}+\mathrm{Na}]^{+}$calc'd for $\mathrm{C}_{16} \mathrm{H}_{18} \mathrm{~N}_{2} \mathrm{NaO}_{3}, 309.1210$, found 309.1217 .

ethyl 5-oxo-4-phenyl-5-(1-tosyl-1H-pyrazol-3-yl)pentanoate (4o)<smiles>CCOC(=O)CCC(C(=O)c1ccn(C)n1)c1ccccc1</smiles>

$\mathrm{PE} / \mathrm{EA}=3 / 1$. Colorless oil, 54.1mg, 90\% 
${ }^{1} \mathrm{H}$ NMR (400 MHz, $\left.\mathrm{CDCl}_{3}\right) \delta 7.41(\mathrm{~d}, J=7.6 \mathrm{~Hz}, 2 \mathrm{H}), 7.33-7.28(\mathrm{~m}, 3 \mathrm{H}), 7.21(\mathrm{t}, J$ $=7.2 \mathrm{~Hz}, 1 \mathrm{H}), 6.75(\mathrm{~s}, 1 \mathrm{H}), 4.89(\mathrm{t}, J=7.3 \mathrm{~Hz}, 1 \mathrm{H}), 4.11(\mathrm{q}, J=7.1 \mathrm{~Hz}, 2 \mathrm{H}), 3.95(\mathrm{~s}$, $3 \mathrm{H}), 2.51-2.42(\mathrm{~m}, 1 \mathrm{H}), 2.37-2.15(\mathrm{~m}, 3 \mathrm{H}), 1.24(\mathrm{t}, J=7.1 \mathrm{~Hz}, 3 \mathrm{H})$.

${ }^{13} \mathrm{C} \mathrm{NMR}\left(100 \mathrm{MHz}, \mathrm{CDCl}_{3}\right) \delta 194.9,173.2,150.5,138.9,131.7,128.7,128.6,127.0$, $107.7,60.3,52.2,39.6,32.3,28.0,14.2$.

HRMS-ESI $(\mathrm{m} / \mathrm{z})[\mathrm{M}+\mathrm{H}]^{+}$calc'd for $\mathrm{C}_{17} \mathrm{H}_{21} \mathrm{~N}_{2} \mathrm{O}_{3}, 301.1547$, found 301.1553 .

ethyl 5-(benzofuran-2-yl)-5-oxo-4-phenylpentanoate (4p)

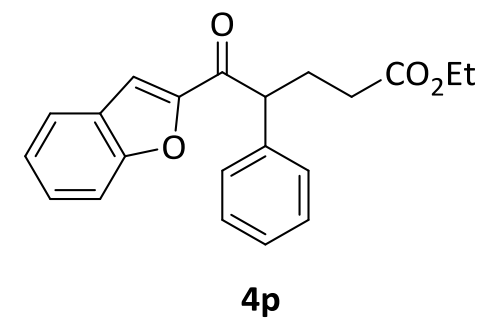

$\mathrm{PE} / \mathrm{EA}=30 / 1$. Colorless oil, $41.5 \mathrm{mg}, 62 \%$

${ }^{1} \mathrm{H}$ NMR $\left(400 \mathrm{MHz}, \mathrm{CDCl}_{3}\right) \delta 7.67(\mathrm{~d}, J=7.9 \mathrm{~Hz}, 1 \mathrm{H}), 7.56(\mathrm{~d}, J=8.4 \mathrm{~Hz}, 1 \mathrm{H}), 7.51$ (s, 1H), 7.47 (dd, $J=11.4,4.1 \mathrm{~Hz}, 1 \mathrm{H}), 7.39$ (d, $J=7.3 \mathrm{~Hz}, 2 \mathrm{H}), 7.33$ (dd, $J=13.3$, $5.5 \mathrm{~Hz}, 2 \mathrm{H}), 7.28-7.22(\mathrm{~m}, 2 \mathrm{H}), 4.64(\mathrm{t}, J=7.4 \mathrm{~Hz}, 1 \mathrm{H}), 4.14(\mathrm{q}, J=7.1 \mathrm{~Hz}, 2 \mathrm{H})$, $2.56-2.47(\mathrm{~m}, 1 \mathrm{H}), 2.38-2.31(\mathrm{~m}, 2 \mathrm{H}), 2.26-2.17(\mathrm{~m}, 1 \mathrm{H}), 1.26(\mathrm{t}, J=7.1 \mathrm{~Hz}$, $3 \mathrm{H})$.

${ }^{13} \mathrm{C}$ NMR $\left(100 \mathrm{MHz}, \mathrm{CDCl}_{3}\right) \delta 190.2,173.2,155.6,152.1,138.1,129.0,128.4,128.3$, $127.5,127.0,123.9,123.3,113.9,112.5,60.4,53.0,31.8,27.9,14.2$.

HRMS-ESI $(\mathrm{m} / \mathrm{z})[\mathrm{M}+\mathrm{H}]^{+}$calc'd for $\mathrm{C}_{21} \mathrm{H}_{21} \mathrm{O}_{4}, 337.1434$, found 337.1428.

ethyl 5-(4-fluorophenyl)-4-(4-methoxyphenyl)-5-oxopentanoate (5a)<smiles>CCOC(=O)CCC(C(=O)c1ccc(F)cc1)c1ccc(OC)cc1</smiles>

5a

$\mathrm{PE} / \mathrm{EA}=30 / 1$. Colorless oil, $64.6 \mathrm{mg}, 94 \%$.

${ }^{1} \mathrm{H}$ NMR $\left(400 \mathrm{MHz}, \mathrm{CDCl}_{3}\right) \delta 8.42-7.55(\mathrm{~m}, 2 \mathrm{H}), 7.33-7.11(\mathrm{~m}, 2 \mathrm{H}), 7.11-6.98$ $(\mathrm{m}, 2 \mathrm{H}), 6.95-6.75(\mathrm{~m}, 2 \mathrm{H}), 4.60(\mathrm{t}, J=7.4 \mathrm{~Hz}, 1 \mathrm{H}), 4.13(\mathrm{q}, J=7.1 \mathrm{~Hz}, 2 \mathrm{H}), 3.77$ (s, 3H), 2.47-2.38 (m, 1H), $2.30(\mathrm{t}, J=6.9 \mathrm{~Hz}, 2 \mathrm{H}), 2.19-2.10(\mathrm{~m}, 1 \mathrm{H}), 1.25(\mathrm{t}, J=$ $7.2 \mathrm{~Hz}, 3 \mathrm{H})$.

${ }^{19} \mathrm{~F} \mathrm{NMR}\left(376 \mathrm{MHz}, \mathrm{CDCl}_{3}\right) \delta-105.46$.

${ }^{13} \mathrm{C} \mathrm{NMR}\left(100 \mathrm{MHz}, \mathrm{CDCl}_{3}\right) \delta 198.0,173.4,165.5(\mathrm{~d}, \mathrm{~J}=254.9 \mathrm{~Hz}), 158.8,133.0(\mathrm{~d}$, $J=3.1 \mathrm{~Hz}), 131.4(\mathrm{~d}, J=9.2 \mathrm{~Hz}), 130.6,129.3,115.6(\mathrm{~d}, J=21.9 \mathrm{~Hz}), 114.5,60.4$, 55.2, 51.5, 31.7, 28.7, 14.2 .

HRMS-ESI $(\mathrm{m} / \mathrm{z})[\mathrm{M}+\mathrm{H}]^{+}$calc'd for $\mathrm{C}_{20} \mathrm{H}_{22} \mathrm{FO}_{4}, 345.1497$, found 345.1507. 
ethyl 4-(4-bromophenyl)-5-(4-fluorophenyl)-5-oxopentanoate (5b)<smiles>CCOC(=O)CCC(C(=O)c1ccc(F)cc1)c1ccc(Br)cc1</smiles>

$5 b$

$\mathrm{PE} / \mathrm{EA}=30 / 1$. Colorless oil, $63.5 \mathrm{mg}, 81 \%$.

${ }^{1} \mathrm{H}$ NMR (400 MHz, $\left.\mathrm{CDCl}_{3}\right) \delta 8.25-7.72(\mathrm{~m}, 2 \mathrm{H}), 7.44-7.47(\mathrm{~m}, 2 \mathrm{H}), 7.27-7.15$ (m, 2H), $7.15-6.95(\mathrm{~m}, 2 \mathrm{H}), 4.66(\mathrm{t}, J=7.3 \mathrm{~Hz}, 1 \mathrm{H}), 4.13$ (q, $J=7.1 \mathrm{~Hz}, 2 \mathrm{H}), 2.47-$ $2.42(\mathrm{~m}, 1 \mathrm{H}), 2.30(\mathrm{t}, J=6.9 \mathrm{~Hz}, 2 \mathrm{H}), 2.16-2.11(\mathrm{~m}, 1 \mathrm{H}), 1.25(\mathrm{t}, J=7.1 \mathrm{~Hz}, 3 \mathrm{H})$.

${ }^{19} \mathrm{~F}$ NMR $\left(376 \mathrm{MHz}, \mathrm{CDCl}_{3}\right) \delta-104.74$.

${ }^{13} \mathrm{C}$ NMR $\left(100 \mathrm{MHz}, \mathrm{CDCl}_{3}\right) \delta 197.4,173.1,165.7(\mathrm{~d}, J=255.4 \mathrm{~Hz}), 137.7,132.7$, 132.3, $131.4(\mathrm{~d}, J=9.3 \mathrm{~Hz}), 130.0,121.5,115.8(\mathrm{~d}, J=21.8 \mathrm{~Hz}), 60.5,51.6,31.5$, 28.6, 14.2 .

HRMS-ESI $(\mathrm{m} / \mathrm{z})[\mathrm{M}+\mathrm{H}]^{+}$calc'd for $\mathrm{C}_{19} \mathrm{H}_{19} \mathrm{BrFO}_{3}, 393.0496$, found 393.0489.

ethyl 4-(4-cyanophenyl)-5-(4-fluorophenyl)-5-oxopentanoate (5c)<smiles>CCOC(=O)CCC(C(=O)c1ccc(F)cc1)c1ccc(C#N)cc1</smiles>

$\mathrm{PE} / \mathrm{EA}=30 / 1$. Colorless oil, $56.1 \mathrm{mg}, 83 \%$.

${ }^{1} \mathrm{H}$ NMR (400 MHz, $\left.\mathrm{CDCl}_{3}\right) \delta 8.06-7.86(\mathrm{~m}, 2 \mathrm{H}), 7.63(\mathrm{~d}, J=7.9 \mathrm{~Hz}, 2 \mathrm{H}), 7.44(\mathrm{~d}$, $J=7.8 \mathrm{~Hz}, 2 \mathrm{H}), 7.11(\mathrm{t}, J=8.4 \mathrm{~Hz}, 2 \mathrm{H}), 4.80(\mathrm{t}, J=7.3 \mathrm{~Hz}, 1 \mathrm{H}), 4.14(\mathrm{q}, J=7.1 \mathrm{~Hz}$, $2 \mathrm{H}), 2.53-2.45(\mathrm{~m}, 1 \mathrm{H}), 2.30(\mathrm{t}, J=6.9 \mathrm{~Hz}, 2 \mathrm{H}), 2.19-2.10(\mathrm{~m}, 1 \mathrm{H}), 1.25(\mathrm{t}, J=7.1$ $\mathrm{Hz}, 3 \mathrm{H})$.

${ }^{19} \mathrm{~F}$ NMR $\left(376 \mathrm{MHz}, \mathrm{CDCl}_{3}\right) \delta-104.02$.

${ }^{13} \mathrm{C}$ NMR $\left(100 \mathrm{MHz}, \mathrm{CDCl}_{3}\right) \delta 196.8,172.9,165.9(\mathrm{~d}, J=256.1 \mathrm{~Hz}), 144.0,132.9$, $132.5(\mathrm{~d}, J=3.0 \mathrm{~Hz}), 131.4(\mathrm{~d}, J=9.5 \mathrm{~Hz}), 129.1,118.5,116(\mathrm{~d}, J=22.0 \mathrm{~Hz}), 111.5$, 60.6, 52.0, 31.4, 28.7, 14.2 .

HRMS-ESI (m/z) $[\mathrm{M}+\mathrm{H}]^{+}$calc'd for $\mathrm{C}_{20} \mathrm{H}_{19} \mathrm{FNO}_{3}, 340.1343$, found 340.1349 .

ethyl 5-(4-fluorophenyl)-5-oxo-4-(m-tolyl)pentanoate (5d) 
<smiles>CCOC(=O)CCC(C(=O)c1ccc(F)cc1)c1cccc(C)c1</smiles>

5d

$\mathrm{PE} / \mathrm{EA}=30 / 1$. Colorless oil, $63.2 \mathrm{mg}, 96 \%$.

${ }^{1} \mathrm{H}$ NMR $\left(400 \mathrm{MHz}, \mathrm{CDCl}_{3}\right) \delta 8.13-7.75(\mathrm{~m}, 2 \mathrm{H}), 7.23-7.18(\mathrm{~m}, 1 \mathrm{H}), 7.11-7.00$ $(\mathrm{m}, 5 \mathrm{H}), 4.61$ (t, $J=7.3 \mathrm{~Hz}, 1 \mathrm{H}), 4.13(\mathrm{q}, J=7.1 \mathrm{~Hz}, 2 \mathrm{H}), 2.49-2.40(\mathrm{~m}, 1 \mathrm{H}), 2.32$ (s, 3H), $2.30(\mathrm{~d}, J=7.3 \mathrm{~Hz}, 2 \mathrm{H}), 2.22-2.10(\mathrm{~m}, 1 \mathrm{H}), 1.25$ (t, $J=7.1 \mathrm{~Hz}, 3 \mathrm{H})$.

${ }^{19} \mathrm{~F} \mathrm{NMR}\left(376 \mathrm{MHz}, \mathrm{CDCl}_{3}\right) \delta-105.39$.

${ }^{13} \mathrm{C}$ NMR $\left(100 \mathrm{MHz}, \mathrm{CDCl}_{3}\right) \delta 197.8,173.3,165.6(\mathrm{~d}, J=255.4 \mathrm{~Hz}), 138.9,138.6$, $131.4(\mathrm{~d}, J=9.5 \mathrm{~Hz}), 129.0,128.7,128.2,125.4,115.6(\mathrm{~d}, J=21.7 \mathrm{~Hz}), 60.4,52.4$, 31.8, 28.8, 21.5, 14.3.

HRMS-ESI (m/z) $[\mathrm{M}+\mathrm{H}]^{+}$calc'd for $\mathrm{C}_{20} \mathrm{H}_{22} \mathrm{FO}_{3}, 329.1547$, found 329.1550.

ethyl 4-(3-bromophenyl)-5-(4-fluorophenyl)-5-oxopentanoate (5e)<smiles>CCOC(=O)CCC(C(=O)c1ccc(F)cc1)c1cccc(Br)c1</smiles>

$5 e$

$\mathrm{PE} / \mathrm{EA}=30 / 1$. Colorless oil, $72.9 \mathrm{mg}, 93 \%$.

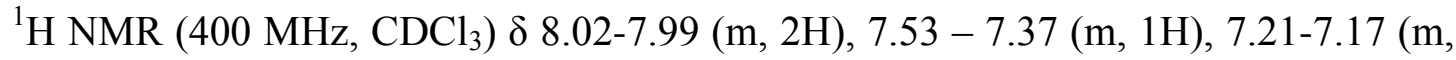
$3 \mathrm{H}), 7.08-7.05(\mathrm{~m}, 2 \mathrm{H}), 5.17(\mathrm{t}, J=6.9 \mathrm{~Hz}, 1 \mathrm{H}), 4.13(\mathrm{t}, J=7.3 \mathrm{~Hz}, 2 \mathrm{H}), 2.64-2.25$ $(\mathrm{m}, 3 \mathrm{H}), 2.17-2.10(\mathrm{~m}, 1 \mathrm{H}), 1.25(\mathrm{t}, J=7.2 \mathrm{~Hz}, 3 \mathrm{H})$.

${ }^{19} \mathrm{~F}$ NMR $\left(376 \mathrm{MHz}, \mathrm{CDCl}_{3}\right) \delta-104.85$.

${ }^{13} \mathrm{C}$ NMR $\left(100 \mathrm{MHz}, \mathrm{CDCl}_{3}\right) \delta 197.4,173.1,165.7(\mathrm{~d}, J=255.3 \mathrm{~Hz}), 136.6,133.6$, $132.6(\mathrm{~d}, J=3.0 \mathrm{~Hz}), 131.3(\mathrm{~d}, J=9.3 \mathrm{~Hz}), 130.1,128.9,128.8,127.7,115.8(\mathrm{~d}, J=$ $21.8 \mathrm{~Hz}), 60.5,48.0,31.6,28.3,14.3$.

HRMS-ESI $(\mathrm{m} / \mathrm{z})[\mathrm{M}+\mathrm{H}]^{+}$calc'd for $\mathrm{C}_{19} \mathrm{H}_{19} \mathrm{BrFO}_{3}, 393.0496$, found 393.0488.

ethyl 4-(2-chlorophenyl)-5-(4-fluorophenyl)-5-oxopentanoate (5f)<smiles>CCOC(=O)CCC(C(=O)c1ccc(F)cc1)c1ccccc1Cl</smiles>

$5 f$

$\mathrm{PE} / \mathrm{EA}=30 / 1$. Colorless oil, $55.6 \mathrm{mg}, 80 \%$.

${ }^{1} \mathrm{H}$ NMR $\left(400 \mathrm{MHz}, \mathrm{CDCl}_{3}\right) \delta 8.04-7.90(\mathrm{~m}, 2 \mathrm{H}), 7.47-7.45(\mathrm{~m}, 1 \mathrm{H}), 7.39(\mathrm{dd}, J=$ 
7.6, $1.8 \mathrm{~Hz}, 1 \mathrm{H}), 7.27-7.15(\mathrm{~m}, 2 \mathrm{H}), 7.16-7.03(\mathrm{~m}, 2 \mathrm{H}), 4.66(\mathrm{t}, J=7.3 \mathrm{~Hz}, 1 \mathrm{H})$, $4.14(\mathrm{dt}, J=7.1,6.0 \mathrm{~Hz}, 2 \mathrm{H}), 2.49-2.10(\mathrm{~m}, 1 \mathrm{H}), 2.30(\mathrm{t}, J=6.9 \mathrm{~Hz}, 2 \mathrm{H}), 2.19-$ $2.10(\mathrm{~m}, 1 \mathrm{H}), 1.26(\mathrm{t}, J=7.1 \mathrm{~Hz}, 3 \mathrm{H})$.

${ }^{19} \mathrm{~F}$ NMR $\left(376 \mathrm{MHz}, \mathrm{CDCl}_{3}\right) \delta-104.66(\mathrm{~s})$.

${ }^{13} \mathrm{C}$ NMR $\left(100 \mathrm{MHz}, \mathrm{CDCl}_{3}\right) \delta 197.2,173.1,165.7(\mathrm{~d}, J=255.4 \mathrm{~Hz}), 140.9,132.7(\mathrm{~d}$, $J=2.9 \mathrm{~Hz}), 131.4(\mathrm{~d}, J=9.5 \mathrm{~Hz}), 131.2,130.6,126.9,123.1,115.8(\mathrm{~d}, J=21.9 \mathrm{~Hz})$, $60.5,51.7,31.6,28.8,14.2$.

HRMS-ESI (m/z) $[\mathrm{M}+\mathrm{H}]^{+}$calc'd for $\mathrm{C}_{19} \mathrm{H}_{19} \mathrm{ClFO}_{3}, 349.1001$, found 349.0993.

ethyl 5-(4-fluorophenyl)-5-oxo-4-(o-tolyl)pentanoate (5g)<smiles>CCOC(=O)CCC(C(=O)c1ccc(F)cc1)c1ccccc1C</smiles>

$5 g$

$\mathrm{PE} / \mathrm{EA}=30 / 1$. Colorless oil, $60.3 \mathrm{mg}, 92 \%$.

${ }^{1} \mathrm{H}$ NMR $\left(400 \mathrm{MHz}, \mathrm{CDCl}_{3}\right) \delta 8.00-7.76(\mathrm{~m}, 2 \mathrm{H}), 7.24-7.22(\mathrm{~m}, 1 \mathrm{H}), 7.18-7.08$ $(\mathrm{m}, 2 \mathrm{H}), 7.08-6.86(\mathrm{~m}, 3 \mathrm{H}), 4.82(\mathrm{t}, J=6.7 \mathrm{~Hz}, 1 \mathrm{H}), 4.13(\mathrm{q}, J=7.2 \mathrm{~Hz}, 2 \mathrm{H}), 2.54$ $(\mathrm{s}, 3 \mathrm{H}), 2.50-2.27(\mathrm{~m}, 3 \mathrm{H}), 2.09-2.01(\mathrm{~m}, 1 \mathrm{H}), 1.25(\mathrm{t}, J=7.1 \mathrm{~Hz}, 3 \mathrm{H})$.

${ }^{19} \mathrm{~F} \mathrm{NMR}\left(376 \mathrm{MHz}, \mathrm{CDCl}_{3}\right) \delta-105.46$.

${ }^{13} \mathrm{C}$ NMR $\left(100 \mathrm{MHz}, \mathrm{CDCl}_{3}\right) \delta 198.4,173.4,165.5(\mathrm{~d}, J=254.7 \mathrm{~Hz}), 137.5,135.2$, $133.3(\mathrm{~d}, J=3.0 \mathrm{~Hz}), 131.2,131.1(\mathrm{~d}, J=9.2 \mathrm{~Hz}), 127.3,127.3,126.8,115.6$ (d, $J=$ $21.9 \mathrm{~Hz}), 60.4,48.5,31.9,28.2,19.7,14.2$.

HRMS-ESI $(\mathrm{m} / \mathrm{z})[\mathrm{M}+\mathrm{H}]^{+}$calc'd for $\mathrm{C}_{20} \mathrm{H}_{22} \mathrm{FO}_{3}, 329.1547$, found 329.1556.

ethyl 5-(4-fluorophenyl)-4-(naphthalen-1-yl)-5-oxopentanoate (5h)<smiles>CCOC(=O)CCC(C(=O)c1ccc(F)cc1)c1cccc2ccccc12</smiles>

$5 \mathrm{~h}$

$\mathrm{PE} / \mathrm{EA}=30 / 1$. Colorless oil, $68.5 \mathrm{mg}, 94 \%$.

${ }^{1} \mathrm{H}$ NMR $\left(400 \mathrm{MHz}, \mathrm{CDCl}_{3}\right) \delta 8.42(\mathrm{~d}, J=8.6 \mathrm{~Hz}, 1 \mathrm{H}), 7.96-7.87(\mathrm{~m}, 3 \mathrm{H}), 7.76(\mathrm{~d}$, $J=8.2 \mathrm{~Hz}, 1 \mathrm{H}), 7.69(\mathrm{t}, J=7.5 \mathrm{~Hz}, 1 \mathrm{H}), 7.58(\mathrm{t}, J=7.5 \mathrm{~Hz}, 1 \mathrm{H}), 7.36(\mathrm{t}, J=7.7 \mathrm{~Hz}$, $1 \mathrm{H}), 7.25(\mathrm{~d}, J=7.1 \mathrm{~Hz}, 1 \mathrm{H}), 6.95(\mathrm{t}, J=8.6 \mathrm{~Hz}, 2 \mathrm{H}), 5.50(\mathrm{dd}, J=8.2,5.7 \mathrm{~Hz}, 1 \mathrm{H})$, $4.15(\mathrm{q}, J=7.1 \mathrm{~Hz}, 2 \mathrm{H}), 2.63-2.54(\mathrm{~m} \mathrm{1H}), 2.44(\mathrm{t}, J=6.8 \mathrm{~Hz}, 2 \mathrm{H}), 2.30-2.22(\mathrm{~m}$, $1 \mathrm{H}), 1.25(\mathrm{t}, J=7.1 \mathrm{~Hz}, 3 \mathrm{H})$.

${ }^{19} \mathrm{~F} \mathrm{NMR}\left(376 \mathrm{MHz}, \mathrm{CDCl}_{3}\right) \delta-105.29(\mathrm{~s})$.

${ }^{13} \mathrm{C}$ NMR $\left(100 \mathrm{MHz}, \mathrm{CDCl}_{3}\right) \delta 198.0,173.6,165.5(\mathrm{~d}, J=254.9 \mathrm{~Hz}), 135.5,134.4$, $133.0(\mathrm{~d}, J=3.0 \mathrm{~Hz}), 131.1(\mathrm{~d}, J=9.3 \mathrm{~Hz}), 131.0,129.4,128.1,127.1,126.0,125.7$, 
$125.5,122.6,115.6(\mathrm{~d}, J=21.9 \mathrm{~Hz}), 60.5,47.4,31.9,28.5,14.2$.

HRMS-ESI $(\mathrm{m} / \mathrm{z})[\mathrm{M}+\mathrm{H}]^{+}$calc'd for $\mathrm{C}_{23} \mathrm{H}_{22} \mathrm{FO}_{3}, 365.1547$, found 365.1551 .

ethyl 5-(4-fluorophenyl)-4-(naphthalen-2-yl)-5-oxopentanoate (5i)<smiles>CCOC(=O)CCC(C(=O)c1ccc(F)cc1)c1ccc2ccccc2c1</smiles>

$\mathrm{PE} / \mathrm{EA}=30 / 1$. Colorless oil, $67.0 \mathrm{mg}, 92 \%$.

${ }^{1} \mathrm{H}$ NMR (400 MHz, $\left.\mathrm{CDCl}_{3}\right) \delta 8.10-7.94(\mathrm{~m}, 2 \mathrm{H}), 7.87-7.77(\mathrm{~m}, 3 \mathrm{H}), 7.74(\mathrm{~d}, J=$ $1.8 \mathrm{~Hz}, 1 \mathrm{H}), 7.57-7.39(\mathrm{~m}, 3 \mathrm{H}), 7.05(\mathrm{t}, J=8.6 \mathrm{~Hz}, 2 \mathrm{H}), 4.84(\mathrm{t}, J=7.1 \mathrm{~Hz}, 1 \mathrm{H})$, $4.13(\mathrm{q}, J=7.2 \mathrm{~Hz}, 2 \mathrm{H}), 2.67-2.46(\mathrm{~m}, 1 \mathrm{H}), 2.42-2.10(\mathrm{~m}, 3 \mathrm{H}), 1.25(\mathrm{t}, J=7.1 \mathrm{~Hz}$, $3 \mathrm{H})$.

${ }^{19} \mathrm{~F} \mathrm{NMR}\left(376 \mathrm{MHz}, \mathrm{CDCl}_{3}\right) \delta-105.14$.

${ }^{13} \mathrm{C}$ NMR $\left(100 \mathrm{MHz}, \mathrm{CDCl}_{3}\right) \delta 197.7,173.3,165.6(\mathrm{~d}, J=255.0 \mathrm{~Hz}), 136.2,133.6$, $132.9(\mathrm{~d}, J=3.0 \mathrm{~Hz}), 132.6,131.5(\mathrm{~d}, J=9.4 \mathrm{~Hz}), 129.1,127.8,127.7,127.3,126.4$, $126.1,126.0,115.7$ (d, $J=21.9 \mathrm{~Hz}), 60.4,52.5,31.7,28.7,14.2$.

HRMS-ESI $(\mathrm{m} / \mathrm{z})[\mathrm{M}+\mathrm{H}]^{+}$calc'd for $\mathrm{C}_{23} \mathrm{H}_{22} \mathrm{FO}_{3}, 365.1547$, found 365.1553 .

ethyl 5-(4-fluorophenyl)-5-oxo-4-(quinolin-5-yl)pentanoate (5j)<smiles>CCOC(=O)CCC(C(=O)c1ccc(F)cc1)c1cccc2ncccc12</smiles>

$5 \mathbf{j}$

$\mathrm{PE} / \mathrm{EA}=5 / 1$. Colorless oil, $62.6 \mathrm{mg}, 86 \%$.

${ }^{1} \mathrm{H}$ NMR $\left(400 \mathrm{MHz}, \mathrm{CDCl}_{3}\right) \delta 8.90(\mathrm{~d}, J=3.3 \mathrm{~Hz}, 1 \mathrm{H}), 8.17-8.10(\mathrm{~m}, 2 \mathrm{H}), 8.06-$ $8.01(\mathrm{~m}, 2 \mathrm{H}), 7.73(\mathrm{~s}, 1 \mathrm{H}), 7.70(\mathrm{~s}, 1 \mathrm{H}), 7.42(\mathrm{dd}, J=8.1,3.9 \mathrm{~Hz}, 1 \mathrm{H}), 7.07(\mathrm{t}, J=8.0$ $\mathrm{Hz}, 2 \mathrm{H}), 4.90(\mathrm{t}, J=7.0 \mathrm{~Hz}, 1 \mathrm{H}), 4.13(\mathrm{q}, J=7.1 \mathrm{~Hz}, 2 \mathrm{H}), 2.58-2.52(\mathrm{~m}, 1 \mathrm{H}), 2.37-$ $2.32(\mathrm{~m}, 2 \mathrm{H}), 2.30-2.20(\mathrm{~m}, 1 \mathrm{H}), 1.24(\mathrm{t}, J=7.0 \mathrm{~Hz}, 3 \mathrm{H})$.

${ }^{19} \mathrm{~F} \mathrm{NMR}\left(376 \mathrm{MHz}, \mathrm{CDCl}_{3}\right) \delta-104.69(\mathrm{~s})$.

${ }^{13} \mathrm{C}$ NMR $\left(100 \mathrm{MHz}, \mathrm{CDCl}_{3}\right) \delta 197.4,173.1,165.7$ (d, $\left.J=255.5 \mathrm{~Hz}\right), 150.3,147.2$, 137.2, 136.2, 132.8 (d, $J=3.0 \mathrm{~Hz}), 131.4$ (d, $J=9.4 \mathrm{~Hz}), 130.3,130.0,128.5,127.0$, $121.5,115.8(\mathrm{~d}, J=21.9 \mathrm{~Hz}), 60.5,52.1,31.7,28.9,14.2$.

HRMS-ESI $(\mathrm{m} / \mathrm{z})[\mathrm{M}+\mathrm{Na}]^{+}$calc'd for $\mathrm{C}_{22} \mathrm{H}_{20} \mathrm{FNNaO}_{3}, 388.1319$, found 388.1314.

ethyl 5-(4-fluorophenyl)-4-(isoquinolin-3-yl)-5-oxopentanoate (5k) 
<smiles>CCOC(=O)CCC(C(=O)c1ccc(F)cc1)c1cc2ccccc2cn1</smiles>

$\mathrm{PE} / \mathrm{EA}=5 / 1$ to $3 / 1$. Colorless oil, $43.7 \mathrm{mg}, 60 \%$.

${ }^{1} \mathrm{H}$ NMR (400 MHz, MeOD) $\delta 8.28(\mathrm{~d}, J=8.5 \mathrm{~Hz}, 1 \mathrm{H}), 8.17-8.09(\mathrm{~m}, 2 \mathrm{H}), 8.03(\mathrm{~d}$, $J=8.5 \mathrm{~Hz}, 1 \mathrm{H}), 7.88(\mathrm{~d}, J=8.1 \mathrm{~Hz}, 1 \mathrm{H}), 7.75(\mathrm{dd}, J=7.5,7.7 \mathrm{~Hz}, 1 \mathrm{H}), 7.57(\mathrm{dd}, J=$ 7.5, $7.7 \mathrm{~Hz}, 1 \mathrm{H}), 7.51(\mathrm{~d}, J=8.5 \mathrm{~Hz}, 1 \mathrm{H}), 7.15(\mathrm{t}, J=8.4 \mathrm{~Hz}, 2 \mathrm{H}), 5.14(\mathrm{t}, J=6.7 \mathrm{~Hz}$, $1 \mathrm{H}), 4.06(\mathrm{q}, J=7.0 \mathrm{~Hz}, 2 \mathrm{H}), 2.68-2.53(\mathrm{~m}, 1 \mathrm{H}), 2.48-2.22(\mathrm{~m}, 3 \mathrm{H}), 1.18(\mathrm{t}, J=$ $7.1 \mathrm{~Hz}, 3 \mathrm{H})$.

${ }^{19}$ F NMR (376 MHz, MeOD) $\delta-107.28$ (s).

${ }^{13} \mathrm{C}$ NMR (100 MHz, MeOD) $\delta$ 196.8, 173.3, $165.7(\mathrm{~d}, J=253.8 \mathrm{~Hz}), 159.1,147.6$, $137.7,133.1(\mathrm{~d}, J=3.0 \mathrm{~Hz}), 131.5(\mathrm{~d}, J=9.5 \mathrm{~Hz}), 129.8,127.9,127.5,127.1,126.5$, 120.2, $115.2(\mathrm{~d}, J=22.3 \mathrm{~Hz}), 60.2,55.5,31.3,27.1,13.0$.

HRMS-ESI (m/z) [M+Na] ${ }^{+}$calc'd for $\mathrm{C}_{22} \mathrm{H}_{20} \mathrm{FNNaO}_{3}, 388.1319$, found 388.1323.

ethyl 5-(4-fluorophenyl)-5-oxo-4-(pyridin-3-yl)pentanoate (5l)<smiles>CCOC(=O)CCC(C(=O)c1ccc(F)cc1)c1cccnc1</smiles>

51

$\mathrm{PE} / \mathrm{EA}=5 / 1$ to $3 / 1$. Colorless oil, $45.4 \mathrm{mg}, 72 \%$.

${ }^{1} \mathrm{H}$ NMR $\left(400 \mathrm{MHz}, \mathrm{CDCl}_{3}\right) \delta 8.66(\mathrm{~s}, 1 \mathrm{H}), 8.55(\mathrm{~s}, 1 \mathrm{H}), 8.16-7.92(\mathrm{~m}, 2 \mathrm{H}), 7.83-$ $7.71(\mathrm{~m}, 1 \mathrm{H}), 7.37(\mathrm{t}, J=6.4 \mathrm{~Hz}, 1 \mathrm{H}), 7.10-7.14(\mathrm{~m}, 2 \mathrm{H}), 4.84(\mathrm{t}, J=7.4 \mathrm{~Hz}, 1 \mathrm{H})$, $4.14(\mathrm{qd}, J=7.1,1.7 \mathrm{~Hz}, 2 \mathrm{H}), 2.55-2.46(\mathrm{~m}, 1 \mathrm{H}), 2.31(\mathrm{q}, J=7.1,6.2 \mathrm{~Hz}, 2 \mathrm{H}), 2.19$ $-2.11(\mathrm{~m}, 1 \mathrm{H}), 1.25(\mathrm{t}, J=7.1 \mathrm{~Hz}, 3 \mathrm{H})$.

${ }^{19} \mathrm{~F} \mathrm{NMR}\left(376 \mathrm{MHz}, \mathrm{CDCl}_{3}\right) \delta-103.97$.

${ }^{13} \mathrm{C}$ NMR $\left(100 \mathrm{MHz}, \mathrm{CDCl}_{3}\right) \delta 197.0,172.8,165.9(\mathrm{~d}, J=256.2 \mathrm{~Hz}), 148.7,147.6$, 136.9, 135.1, $132.3(\mathrm{~d}, J=3.0 \mathrm{~Hz}), 131.5$ (d, $J=9.5 \mathrm{~Hz}), 124.4,116.0$ (d, $J=21.7$ $\mathrm{Hz}), 60.7,49.1,31.4,28.8,14.2$.

HRMS-ESI $(\mathrm{m} / \mathrm{z})[\mathrm{M}+\mathrm{H}]^{+}$calc'd for $\mathrm{C}_{18} \mathrm{H}_{19} \mathrm{FNO}_{3}, 316.1343$, found 316.1352 .

ethyl 5-(4-fluorophenyl)-5-oxo-4-(pyridin-4-yl)pentanoate (5m) 
<smiles>CCOC(=O)CCC(C(=O)c1ccc(F)cc1)c1ccncc1</smiles>

$5 \mathrm{~m}$

$\mathrm{PE} / \mathrm{EA}=3 / 1$. Colorless oil, $42.0 \mathrm{mg}, 67 \%$.

${ }^{1} \mathrm{H}$ NMR (400 MHz, $\left.\mathrm{CDCl}_{3}\right) \delta 8.57(\mathrm{~d}, J=4.8 \mathrm{~Hz}, 2 \mathrm{H}), 7.99(\mathrm{dd}, J=8.4,5.4 \mathrm{~Hz}, 2 \mathrm{H})$, $7.45-7.21(\mathrm{~m}, 2 \mathrm{H}), 7.11(\mathrm{t}, J=8.4 \mathrm{~Hz}, 2 \mathrm{H}), 4.73(\mathrm{t}, J=7.3 \mathrm{~Hz}, 1 \mathrm{H}), 4.14$ (q, $J=7.2$ $\mathrm{Hz}, 2 \mathrm{H}), 2.53-2.45(\mathrm{~m}, 1 \mathrm{H}), 2.35-2.17(\mathrm{~m}, 2 \mathrm{H}), 2.20-2.11(\mathrm{~m}, 1 \mathrm{H}), 1.26(\mathrm{t}, J=$ $7.1 \mathrm{~Hz}, 3 \mathrm{H})$.

${ }^{19} \mathrm{~F} \mathrm{NMR}\left(376 \mathrm{MHz}, \mathrm{CDCl}_{3}\right) \delta-104.02$.

${ }^{13} \mathrm{C}$ NMR $\left(100 \mathrm{MHz}, \mathrm{CDCl}_{3}\right) \delta 196.6,172.9,165.9(\mathrm{~d}, J=255.9 \mathrm{~Hz}), 150.2,147.8$, $132.5,131.4(\mathrm{~d}, J=9.4 \mathrm{~Hz}), 123.6,116.0(\mathrm{~d}, J=22.0 \mathrm{~Hz}), 60.6,51.4,31.4,28.4$, 14.2 .

HRMS-ESI $(\mathrm{m} / \mathrm{z})[\mathrm{M}+\mathrm{H}]^{+}$calc'd for $\mathrm{C}_{18} \mathrm{H}_{19} \mathrm{FNO}_{3}, 316.1343$, found 316.1353 .

ethyl 5-(4-fluorophenyl)-5-oxo-4-(thiophen-3-yl)pentanoate (5n)<smiles>CCOC(=O)CCC(C(=O)c1ccc(F)cc1)c1ccsc1</smiles>

$5 n$

$\mathrm{PE} / \mathrm{EA}=20 / 1$. Colorless oil, $47.8 \mathrm{mg}, 75 \%$.

${ }^{1} \mathrm{H}$ NMR $\left(400 \mathrm{MHz}, \mathrm{CDCl}_{3}\right) \delta 8.06-7.91(\mathrm{~m}, 2 \mathrm{H}), 7.28(\mathrm{~s}, 1 \mathrm{H}), 7.15-7.08(\mathrm{~m}, 3 \mathrm{H})$, $7.03(\mathrm{~d}, J=4.8 \mathrm{~Hz}, 1 \mathrm{H}), 4.84(\mathrm{t}, J=7.2 \mathrm{~Hz}, 1 \mathrm{H}), 4.14(\mathrm{q}, J=7.1 \mathrm{~Hz}, 2 \mathrm{H}), 2.46-2.38$ $(\mathrm{m}, 1 \mathrm{H}), 2.32(\mathrm{t}, J=6.7 \mathrm{~Hz}, 2 \mathrm{H}), 2.22-2.14(\mathrm{~m}, 1 \mathrm{H}), 1.25(\mathrm{t}, J=7.1 \mathrm{~Hz}, 3 \mathrm{H})$.

${ }^{19} \mathrm{~F}$ NMR $\left(376 \mathrm{MHz}, \mathrm{CDCl}_{3}\right) \delta-105.10(\mathrm{~s})$.

${ }^{13} \mathrm{C} \mathrm{NMR}\left(100 \mathrm{MHz}, \mathrm{CDCl}_{3}\right) \delta 197.5,173.2,165.6(\mathrm{~d}, J=255.2 \mathrm{~Hz}), 138.7,132.9(\mathrm{~d}$, $J=3.0 \mathrm{~Hz}), 131.3(\mathrm{~d}, J=9.3 \mathrm{~Hz}), 127.1,126.5,122.5,115.7(\mathrm{~d}, J=21.8 \mathrm{~Hz}), 60.4$, $47.4,31.6,28.3,14.2$.

HRMS-ESI $(\mathrm{m} / \mathrm{z})[\mathrm{M}+\mathrm{H}]^{+}$calc'd for $\mathrm{C}_{17} \mathrm{H}_{18} \mathrm{FO}_{3} \mathrm{~S}, 321.0955$, found 321.0959 .

ethyl 4-(benzofuran-3-yl)-5-(4-fluorophenyl)-5-oxopentanoate (5o)<smiles>CCOC(=O)CCC(C(=O)c1ccc(F)cc1)c1coc2ccccc12</smiles> 
$\mathrm{PE} / \mathrm{EA}=30 / 1$. Colorless oil, $58.1 \mathrm{mg}, 82 \%$.

${ }^{1} \mathrm{H}$ NMR (400 MHz, $\left.\mathrm{CDCl}_{3}\right) \delta 8.15-8.05(\mathrm{~m}, 2 \mathrm{H}), 7.50(\mathrm{~d}, J=7.5 \mathrm{~Hz}, 1 \mathrm{H}), 7.45(\mathrm{~d}$, $J=8.0 \mathrm{~Hz}, 1 \mathrm{H}), 7.27-7.17(\mathrm{~m}, 2 \mathrm{H}), 7.13(\mathrm{t}, J=8.1 \mathrm{~Hz}, 2 \mathrm{H}), 6.59(\mathrm{~s}, 1 \mathrm{H}), 4.99(\mathrm{t}, J$ $=7.6 \mathrm{~Hz}, 1 \mathrm{H}), 4.14(\mathrm{q}, J=7.0 \mathrm{~Hz}, 2 \mathrm{H}), 2.56-2.47(\mathrm{~m}, 1 \mathrm{H}), 2.46-2.32(\mathrm{~m}, 3 \mathrm{H})$, $1.26(\mathrm{t}, J=7.0 \mathrm{~Hz}, 3 \mathrm{H})$.

${ }^{19} \mathrm{~F}$ NMR $\left(376 \mathrm{MHz}, \mathrm{CDCl}_{3}\right) \delta-104.42(\mathrm{~s})$.

${ }^{13} \mathrm{C} \mathrm{NMR}\left(100 \mathrm{MHz}, \mathrm{CDCl}_{3}\right) \delta 194.9,173.0,165.9(\mathrm{~d}, J=255.6 \mathrm{~Hz}), 154.8,132.5(\mathrm{~d}$, $J=2.7 \mathrm{~Hz}), 131.5(\mathrm{~d}, J=9.4 \mathrm{~Hz}), 128.4,124.1,122.9,120.8,115.8(\mathrm{~d}, J=21.9 \mathrm{~Hz})$, 111.2, 104.7, 100.0, 60.5, 46.1, 31.4, 26.1, 14.2 .

HRMS-ESI (m/z) $[\mathrm{M}+\mathrm{H}]^{+}$calc'd for $\mathrm{C}_{21} \mathrm{H}_{20} \mathrm{FO}_{4}, 355.1340$, found 355.1336.

ethyl 4-(benzo[b]thiophen-3-yl)-5-(4-fluorophenyl)-5-oxopentanoate (5p)<smiles>CCOC(=O)CCC(C(=O)c1ccc(F)cc1)c1csc2ccccc12</smiles>

$\mathrm{PE} / \mathrm{EA}=30 / 1$. Colorless oil, 58.9mg, 80\%.

${ }^{1} \mathrm{H}$ NMR (400 MHz, $\left.\mathrm{CDCl}_{3}\right) \delta 8.21-8.00(\mathrm{~m}, 2 \mathrm{H}), 7.78(\mathrm{~d}, J=7.6 \mathrm{~Hz}, 1 \mathrm{H}), 7.69(\mathrm{~d}$, $J=7.7 \mathrm{~Hz}, 1 \mathrm{H}), 7.32(\mathrm{q}, J=7.5 \mathrm{~Hz}, 2 \mathrm{H}), 7.20-7.00(\mathrm{~m}, 3 \mathrm{H}), 5.08(\mathrm{t}, J=7.3 \mathrm{~Hz}$, $1 \mathrm{H}), 4.16(\mathrm{q}, J=7.2 \mathrm{~Hz}, 2 \mathrm{H}), 2.62-2.44(\mathrm{~m}, 1 \mathrm{H}), 2.46-2.38(\mathrm{~m}, 2 \mathrm{H}), 2.38-2.25$ $(\mathrm{m}, 1 \mathrm{H}), 1.26(\mathrm{t}, J=7.2 \mathrm{~Hz}, 3 \mathrm{H})$.

${ }^{19} \mathrm{~F}$ NMR $\left(376 \mathrm{MHz}, \mathrm{CDCl}_{3}\right) \delta-104.43(\mathrm{~s})$.

${ }^{13} \mathrm{C}$ NMR $\left(100 \mathrm{MHz}, \mathrm{CDCl}_{3}\right) \delta 196.3,173.1,165.9(\mathrm{~d}, J=255.7 \mathrm{~Hz}), 141.6,139.8$, $139.6,132.4(\mathrm{~d}, J=3.0 \mathrm{~Hz}), 131.5(\mathrm{~d}, J=9.4 \mathrm{~Hz}), 124.4,124.3,123.3,122.9,122.2$, $115.9(\mathrm{~d}, J=21.9 \mathrm{~Hz}), 60.5,47.4,31.4,29.0,14.2$.

HRMS-ESI (m/z) $[\mathrm{M}+\mathrm{H}]^{+}$calc'd for $\mathrm{C}_{21} \mathrm{H}_{20} \mathrm{FO}_{3} \mathrm{~S}, 371.1112$, found 371.1117.

isopropyl 5-(4-fluorophenyl)-5-oxo-4-phenylpentanoate (6a)<smiles>CC(C)OC(=O)CCC(C(=O)c1ccc(F)cc1)c1ccccc1</smiles>

$6 a$

$\mathrm{PE} / \mathrm{EA}=30 / 1$. Colorless oil, 54.2mg, $83 \%$

${ }^{1} \mathrm{H}$ NMR $\left(400 \mathrm{MHz}, \mathrm{CDCl}_{3}\right) \delta 8.00(\mathrm{dd}, J=8.6,5.5 \mathrm{~Hz}, 2 \mathrm{H}), 7.41-7.27(\mathrm{~m}, 4 \mathrm{H})$, $7.24(\mathrm{t}, J=6.7 \mathrm{~Hz}, 1 \mathrm{H}), 7.06(\mathrm{t}, J=8.4 \mathrm{~Hz}, 2 \mathrm{H}), 5.17-4.88(\mathrm{~m}, 1 \mathrm{H}), 4.66(\mathrm{t}, J=7.3$ $\mathrm{Hz}, 1 \mathrm{H}), 2.50-2.41(\mathrm{~m}, 1 \mathrm{H}), 2.28(\mathrm{t}, J=7.0 \mathrm{~Hz}, 2 \mathrm{H}), 2.19-2.12(\mathrm{~m}, 1 \mathrm{H}), 1.23(\mathrm{~d}, J$ $=6.3 \mathrm{~Hz}, 6 \mathrm{H})$.

${ }^{19} \mathrm{~F} \mathrm{NMR}\left(376 \mathrm{MHz}, \mathrm{CDCl}_{3}\right) \delta-105.34$.

${ }^{13} \mathrm{C}$ NMR $\left(100 \mathrm{MHz}, \mathrm{CDCl}_{3}\right) \delta 197.8,172.7,165.6(\mathrm{~d}, J=254.9 \mathrm{~Hz}), 138.7,133.0(\mathrm{~d}$, $J=3.0 \mathrm{~Hz}), 131.4(\mathrm{~d}, J=9.3 \mathrm{~Hz}), 129.1,128.3,127.4,115.6(\mathrm{~d}, J=21.8 \mathrm{~Hz}), 67.7$, 
52.4, 32.1, 28.8, 21.9, 21.8 .

HRMS-ESI $(\mathrm{m} / \mathrm{z})[\mathrm{M}+\mathrm{H}]^{+}$calc'd for $\mathrm{C}_{20} \mathrm{H}_{22} \mathrm{FO}_{3}, 329.1547$, found 329.1541 .

2,4-dimethylpentan-3-yl 5-(4-fluorophenyl)-5-oxo-4-phenylpentanoate (6b)<smiles>CC(C)C(C)C(C)C(=O)CCC(C(=O)c1ccc(F)cc1)c1ccccc1</smiles>

6b

$\mathrm{PE} / \mathrm{EA}=30 / 1$. Colorless oil, $63.5 \mathrm{mg}, 83 \%$

${ }^{1} \mathrm{H}$ NMR $\left(400 \mathrm{MHz}, \mathrm{CDCl}_{3}\right) \delta 8.00(\mathrm{dd}, J=8.7,5.5 \mathrm{~Hz}, 2 \mathrm{H}), 7.38-7.27(\mathrm{~m}, 4 \mathrm{H})$, $7.27-7.20(\mathrm{~m}, 1 \mathrm{H}), 7.06(\mathrm{t}, J=8.6 \mathrm{~Hz}, 2 \mathrm{H}), 4.72(\mathrm{t}, J=7.3 \mathrm{~Hz}, 1 \mathrm{H}), 4.63(\mathrm{t}, J=6.2$ $\mathrm{Hz}, 1 \mathrm{H}), 2.53-2.44(\mathrm{~m}, 1 \mathrm{H}), 2.35(\mathrm{t}, J=6.9 \mathrm{~Hz}, 2 \mathrm{H}), 2.23-2.14(\mathrm{~m}, 1 \mathrm{H}), 1.97-$ $1.81(\mathrm{~m}, 2 \mathrm{H}), 0.94-0.79(\mathrm{~m}, 12 \mathrm{H})$.

${ }^{19} \mathrm{~F}$ NMR $\left(376 \mathrm{MHz}, \mathrm{CDCl}_{3}\right) \delta-105.38(\mathrm{~s})$.

${ }^{13} \mathrm{C}$ NMR $\left(100 \mathrm{MHz}, \mathrm{CDCl}_{3}\right) \delta 197.9,173.3,165.5(\mathrm{~d}, J=254.9 \mathrm{~Hz}), 138.7,133.0(\mathrm{~d}$, $J=3.0 \mathrm{~Hz}), 131.3(\mathrm{~d}, J=9.3 \mathrm{~Hz}), 129.1,128.3,127.4,115.6(\mathrm{~d}, J=21.9 \mathrm{~Hz}), 82.7$, 52.3, 31.6, 29.3, 28.9, 19.6, 19.5, 17.3, 17.2.

HRMS-ESI $(\mathrm{m} / \mathrm{z})[\mathrm{M}+\mathrm{H}]^{+}$calc'd for $\mathrm{C}_{24} \mathrm{H}_{30} \mathrm{FO}_{3}, 385.2173$, found 385.2168.

tert-butyl 5-(4-fluorophenyl)-5-oxo-4-phenylpentanoate (6c)<smiles>CCCCOC(=O)CCC(C(=O)c1ccc(F)cc1)c1ccccc1</smiles>

$6 c$

$\mathrm{PE} / \mathrm{EA}=30 / 1$. Colorless oil, 53.8mg, 78\%

${ }^{1} \mathrm{H}$ NMR $\left(400 \mathrm{MHz}, \mathrm{CDCl}_{3}\right) \delta 8.05-7.96(\mathrm{~m}, 2 \mathrm{H}), 7.36-7.21(\mathrm{~m}, 6 \mathrm{H}), 7.07(\mathrm{dd}, J=$ 9.6, $7.7 \mathrm{~Hz}, 2 \mathrm{H}), 4.65(\mathrm{t}, J=7.3 \mathrm{~Hz}, 1 \mathrm{H}), 2.45-2.39(\mathrm{~m}, 1 \mathrm{H}), 2.26-2.19(\mathrm{~m}, 2 \mathrm{H})$, $2.15-2.10(\mathrm{~m}, 1 \mathrm{H}), 1.45(\mathrm{~s}, 8 \mathrm{H})$.

${ }^{19} \mathrm{~F} \mathrm{NMR}\left(376 \mathrm{MHz}, \mathrm{CDCl}_{3}\right) \delta-105.35(\mathrm{~s})$.

${ }^{13} \mathrm{C} \mathrm{NMR}\left(100 \mathrm{MHz}, \mathrm{CDCl}_{3}\right) \delta 197.8,172.6,165.5(\mathrm{~d}, J=254.9 \mathrm{~Hz}), 138.8,133.0(\mathrm{~d}$, $J=3.1 \mathrm{~Hz}), 131.4(\mathrm{~d}, J=9.3 \mathrm{~Hz}), 129.1,128.3,127.4,115.6(\mathrm{~d}, J=22.0 \mathrm{~Hz}), 80.4$, 52.4, 33.0, 28.9, 28.1.

HRMS-ESI (m/z) $[\mathrm{M}+\mathrm{H}]^{+}$calc'd for $\mathrm{C}_{21} \mathrm{H}_{24} \mathrm{FO}_{3}, 343.1704$, found 343.1712.

(3s,5s,7s)-adamantan-1-yl 5-(4-fluorophenyl)-5-oxo-4-phenylpentanoate (6d)<smiles>CCOC(=O)CCC(C(=O)c1ccccc1)c1ccccc1</smiles>

6d

$\mathrm{PE} / \mathrm{EA}=30 / 1$. Colorless oil, $60.5 \mathrm{mg}, 72 \%$

${ }^{1} \mathrm{H}$ NMR $\left(400 \mathrm{MHz}, \mathrm{CDCl}_{3}\right) \delta 8.00(\mathrm{dd}, J=8.5,5.5 \mathrm{~Hz}, 2 \mathrm{H}), 7.36-7.27(\mathrm{~m}, 4 \mathrm{H})$, 
$7.24(\mathrm{t}, J=6.8 \mathrm{~Hz}, 1 \mathrm{H}), 7.06(\mathrm{t}, J=8.6 \mathrm{~Hz}, 2 \mathrm{H}), 4.66(\mathrm{t}, J=7.2 \mathrm{~Hz}, 1 \mathrm{H}), 2.46-2.38$ $(\mathrm{m}, 1 \mathrm{H}), 2.27-2.14(\mathrm{~m}, 6 \mathrm{H}), 2.14-2.05(\mathrm{~m}, 6 \mathrm{H}), 1.73-1.60(\mathrm{~m}, 6 \mathrm{H})$.

${ }^{19} \mathrm{~F}$ NMR $\left(376 \mathrm{MHz}, \mathrm{CDCl}_{3}\right) \delta-105.46(\mathrm{~s})$.

${ }^{13} \mathrm{C}$ NMR (100 MHz, $\left.\mathrm{CDCl}_{3}\right) \delta 197.9,172.3,165.5(\mathrm{~d}, J=254.8 \mathrm{~Hz}), 138.8,133.1$ $(\mathrm{d}, J=3.0 \mathrm{~Hz}), 131.3(\mathrm{~d}, J=9.3 \mathrm{~Hz}), 129.1,128.3,127.3,115.6(\mathrm{~d}, J=21.9 \mathrm{~Hz})$, 80.5, 52.3, 41.4, 36.2, 33.1, 30.8, 29.0.

HRMS-ESI (m/z) $[\mathrm{M}+\mathrm{H}]^{+}$calc'd for $\mathrm{C}_{27} \mathrm{H}_{30} \mathrm{FO}_{3}, 421.2173$, found 421.2177.

benzyl 5-(4-fluorophenyl)-5-oxo-4-phenylpentanoate (6e)<smiles>O=C(CCC(C(=O)c1ccc(F)cc1)c1ccccc1)OCc1ccccc1</smiles>

$6 e$

$\mathrm{PE} / \mathrm{EA}=30 / 1$. Colorless oil, 63.0mg, 84\%

${ }^{1} \mathrm{H}$ NMR (400 MHz, $\left.\mathrm{CDCl}_{3}\right) \delta 8.00-7.87(\mathrm{~m}, 2 \mathrm{H}), 7.41-7.35(\mathrm{~m}, 5 \mathrm{H}), 7.34-7.29$ (m, 2H), $7.27-7.20(\mathrm{~m}, 3 \mathrm{H}), 7.04(\mathrm{t}, J=8.6 \mathrm{~Hz}, 2 \mathrm{H}), 5.18-5.09(\mathrm{~m}, 2 \mathrm{H}), 4.61$ (t, $J$ $=7.3 \mathrm{~Hz}, 1 \mathrm{H}), 2.53-2.44(\mathrm{~m}, 1 \mathrm{H}), 2.38(\mathrm{t}, J=6.9 \mathrm{~Hz}, 2 \mathrm{H}), 2.24-2.16(\mathrm{~m}, 1 \mathrm{H})$.

${ }^{19} \mathrm{~F} \mathrm{NMR}\left(376 \mathrm{MHz}, \mathrm{CDCl}_{3}\right) \delta-105.26(\mathrm{~s})$.

${ }^{13} \mathrm{C} \mathrm{NMR}\left(100 \mathrm{MHz}, \mathrm{CDCl}_{3}\right) \delta 197.7,173.1,165.5(\mathrm{~d}, J=254.9 \mathrm{~Hz}), 138.5,135.9$, $132.9(\mathrm{~d}, J=2.9 \mathrm{~Hz}), 131.4(\mathrm{~d}, J=9.3 \mathrm{~Hz}), 129.1,128.6,128.4,128.3,1282,127.4$, $115.6(\mathrm{~d}, J=21.8 \mathrm{~Hz}), 66.3,52.2,31.7,28.7$.

HRMS-ESI $(\mathrm{m} / \mathrm{z})[\mathrm{M}+\mathrm{H}]^{+}$calc'd for $\mathrm{C}_{24} \mathrm{H}_{22} \mathrm{FO}_{3}, 377.1547$, found 377.1554.

cyclopropylmethyl 5-(4-fluorophenyl)-5-oxo-4-phenylpentanoate (6f)<smiles>O=C(CCC(C(=O)c1ccc(F)cc1)c1ccccc1)OCC1CC1</smiles>

$\mathrm{PE} / \mathrm{EA}=30 / 1$. Colorless oil, $55.5 \mathrm{mg}, 82 \%$

${ }^{1} \mathrm{H}$ NMR $\left(400 \mathrm{MHz}, \mathrm{CDCl}_{3}\right) \delta 8.00(\mathrm{dd}, J=8.5,5.4 \mathrm{~Hz}, 2 \mathrm{H}), 7.36-7.28(\mathrm{~m}, 4 \mathrm{H})$, $7.27-7.21(\mathrm{~m}, 1 \mathrm{H}), 7.06(\mathrm{t}, J=8.4 \mathrm{~Hz}, 2 \mathrm{H}), 4.67(\mathrm{t}, J=7.3 \mathrm{~Hz}, 1 \mathrm{H}), 3.91(\mathrm{~d}, J=7.4$ $\mathrm{Hz}, 2 \mathrm{H}), 2.52-2.43(\mathrm{~m}, 1 \mathrm{H}), 2.34(\mathrm{t}, J=7.0 \mathrm{~Hz}, 2 \mathrm{H}), 2.23-2.15(\mathrm{~m}, 1 \mathrm{H}), 1.17-1.07$ (m, 1H), $0.83-0.45(\mathrm{~m}, 2 \mathrm{H}), 0.30-0.26(\mathrm{~m}, 2 \mathrm{H})$.

${ }^{19} \mathrm{~F} \mathrm{NMR}\left(376 \mathrm{MHz}, \mathrm{CDCl}_{3}\right) \delta-105.33$.

${ }^{13} \mathrm{C} \mathrm{NMR}\left(100 \mathrm{MHz}, \mathrm{CDCl}_{3}\right) \delta 197.7,173.4,165.6(\mathrm{~d}, J=254.9 \mathrm{~Hz}), 138.7,133.0(\mathrm{~d}$, $J=3.0 \mathrm{~Hz}), 131.4(\mathrm{~d}, J=9.3 \mathrm{~Hz}), 129.1,128.3,127.4,115.6(\mathrm{~d}, J=21.8 \mathrm{~Hz}), 69.2$, $52.4,31.8,28.8,9.8,3.2$.

HRMS-ESI $(\mathrm{m} / \mathrm{z})[\mathrm{M}+\mathrm{H}]^{+}$calc'd for $\mathrm{C}_{21} \mathrm{H}_{22} \mathrm{FO}_{3}, 341.1547$, found 341.1541.

2-(trimethylsilyl)ethyl 5-(4-fluorophenyl)-5-oxo-4-phenylpentanoate (6g) 
<smiles>CCCCCCC(=O)OCCC(=O)c1ccc(F)cc1</smiles>

$6 \mathrm{~g}$

$\mathrm{PE} / \mathrm{EA}=30 / 1$. Colorless oil, $66.2 \mathrm{mg}, 86 \%$

${ }^{1} \mathrm{H}$ NMR $\left(400 \mathrm{MHz}, \mathrm{CDCl}_{3}\right) \delta 8.00(\mathrm{dd}, J=8.5,5.4 \mathrm{~Hz}, 2 \mathrm{H}), 7.37-7.27(\mathrm{~m}, 4 \mathrm{H})$, $7.26-7.24(\mathrm{~m}, 1 \mathrm{H}), 7.06(\mathrm{t}, J=8.5 \mathrm{~Hz}, 2 \mathrm{H}), 4.66(\mathrm{t}, J=7.3 \mathrm{~Hz}, 1 \mathrm{H}), 4.34-4.04(\mathrm{~m}$, $2 \mathrm{H}), 2.50-2.42(\mathrm{~m}, 1 \mathrm{H}), 2.30(\mathrm{t}, J=7.0 \mathrm{~Hz}, 2 \mathrm{H}), 2.20-2.13(\mathrm{~m}, 1 \mathrm{H}), 1.08-0.87$ $(\mathrm{m}, 2 \mathrm{H}), 0.05(\mathrm{~s}, 9 \mathrm{H})$.

${ }^{19} \mathrm{~F} \mathrm{NMR}\left(376 \mathrm{MHz}, \mathrm{CDCl}_{3}\right) \delta-105.36$.

${ }^{13} \mathrm{C} \mathrm{NMR}\left(100 \mathrm{MHz}, \mathrm{CDCl}_{3}\right) \delta 197.7,173.4,165.6(\mathrm{~d}, J=254.9 \mathrm{~Hz}), 138.7,133.0(\mathrm{~d}$, $J=3.0 \mathrm{~Hz}), 131.4(\mathrm{~d}, J=9.3 \mathrm{~Hz}), 129.1,128.3,127.4,115.6(\mathrm{~d}, J=21.7 \mathrm{~Hz}), 62.6$, 52.4, 31.8, 28.8, 17.3.

HRMS-ESI (m/z) [M+Na] ${ }^{+}$calc'd for $\mathrm{C}_{22} \mathrm{H}_{27} \mathrm{FNaO}_{3} \mathrm{Si}$, 409.1606., found 409.1610.

\section{Mechanistic Studies}

(a) Radical trapping experiment

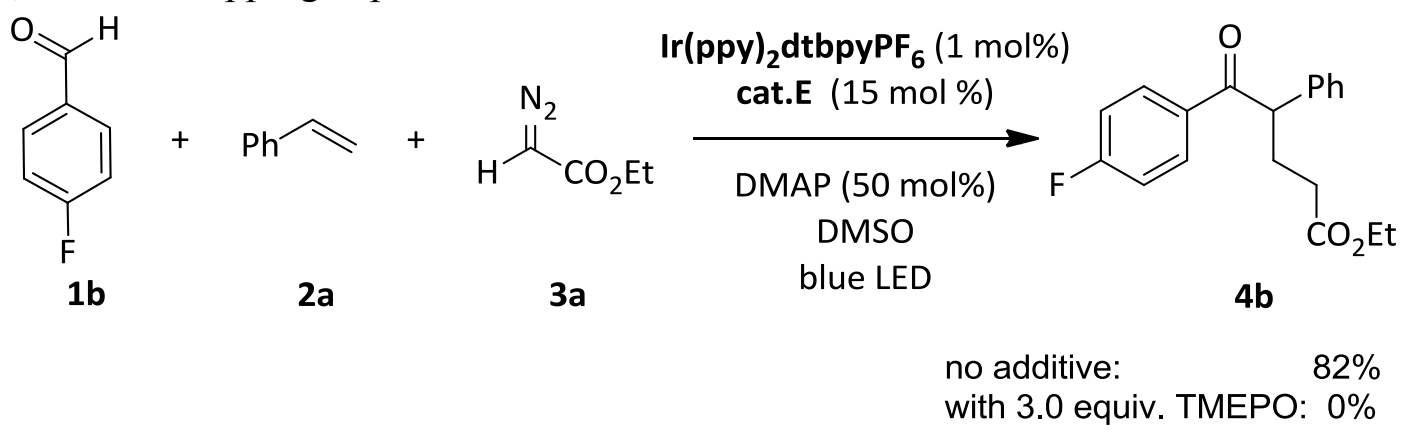

Fig S1. Radical trapping experiment with TEMPO.

In a glovebox, to a flame-dried Schlenk reaction tube equipped with a magnetic stir bar, was added the precatalyst $\mathbf{E}(12 \mathrm{mg}, 0.03 \mathrm{mmol}, 0.15$ equiv), DMAP (12 mg, $0.22 \mathrm{mmol}, 0.5$ equiv), $\operatorname{Ir}(\mathrm{ppy})_{2} \mathrm{dtbpyPF}_{6}(2.0 \mathrm{mg}, 0.002 \mathrm{mmol}, 0.01$ equiv $)$ and DMSO ( $2 \mathrm{~mL}, 0.1 \mathrm{M})$. 1a ( $0.24 \mathrm{mmol}, 1.2$ equiv), $2 \mathbf{a}(0.2 \mathrm{mmol}, 1.0$ equiv), 3a (0.4 mmol, 2.0 equiv), TEMPO (93.8 mg, $0.6 \mathrm{mmol}, 3.0$ equiv) were added subsequently. Then, the tube was caped and taken out of glovebox. The resulting solution was irradiated by a $30 \mathrm{~W}$ blue LED $(450 \mathrm{~nm})$ with stirring at a distance of $3 \mathrm{~cm}$ (with cooling by the fan) at $25^{\circ} \mathrm{C}$ for about $5 \mathrm{~h}$. The desired product $\mathbf{4 b}$ was not detected.

(b) Radical clock experiment 


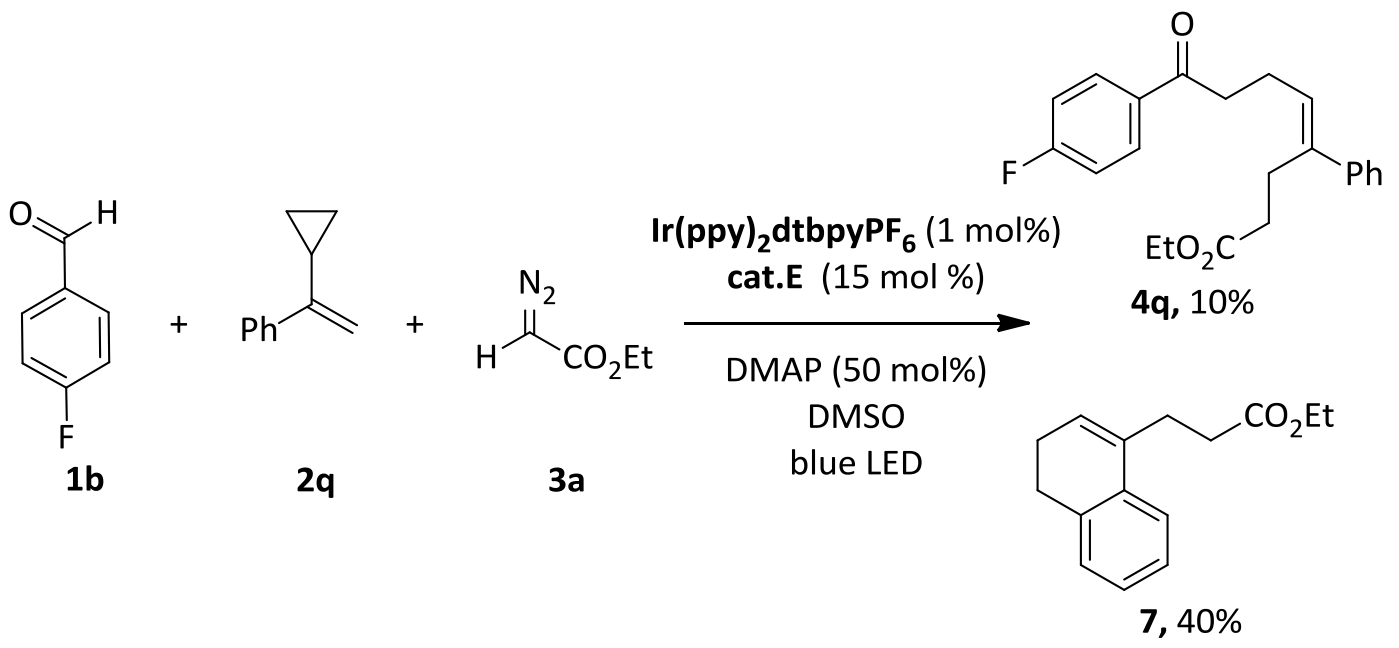

Fig S2. Radical clock experiment.

According to the standard procedure, precatalyst $\mathbf{E}(12 \mathrm{mg}, 0.03 \mathrm{mmol}, 0.15$ equiv), DMAP (12 mg, $0.22 \mathrm{mmol}, 0.5$ equiv), $\operatorname{Ir}(\mathrm{ppy})_{2} \mathrm{dtbpyPF}_{6}(2.0 \mathrm{mg}, 0.002 \mathrm{mmol}, 0.01$ equiv) and DMSO ( $2 \mathrm{~mL}, 0.1 \mathrm{M})$ were added first. $\mathbf{1 a}(0.24 \mathrm{mmol}, 1.2$ equiv), 2q (0.2 mmol, 1.0 equiv), 3a ( $0.4 \mathrm{mmol}, 0.2$ equiv) were added subsequently. Then, the tube was caped and taken out of the glovebox. The resulting solution was irradiated by a $30 \mathrm{~W}$ blue LED $(450 \mathrm{~nm}) \mathrm{f}$ with stirring at a distance of $3 \mathrm{~cm}$ (with cooling by the fan) at $25^{\circ} \mathrm{C}$ for about $5 \mathrm{~h}$. The mixture was diluted with EtOAc, washed with water and dried by $\mathrm{Na}_{2} \mathrm{SO}_{4}$. The organic phase was then concentrated under reduced pressure and purified by column chromatography on silica gel (hexane/EtOAc) to afford the product $\mathbf{4 q}$ and $\mathbf{7 .}$

ethyl (Z)-8-(4-fluorophenyl)-8-oxo-4-phenyloct-4-enoate (4q)<smiles>CCOC(=O)CC/C(=C/CCC(=O)c1ccc(F)cc1)c1ccccc1</smiles>

$\mathrm{PE} / \mathrm{EA}=50 / 1$. Colorless oil, $6.5 \mathrm{mg}, 10 \%$

$E / Z=3 / 7$

${ }^{1} \mathrm{H}$ NMR (400 MHz, $\left.\mathrm{CDCl}_{3}\right) \delta 8.11-7.84(\mathrm{~m}, 2 \mathrm{H}), 7.42-7.20(\mathrm{~m}, 4 \mathrm{H}), 7.20-7.03$ $(\mathrm{m}, 3 \mathrm{H}), 5.73(\mathrm{t}, J=7.4 \mathrm{~Hz}, 0.7 \mathrm{H}, E-\mathrm{H}), 5.58(\mathrm{t}, J=7.2 \mathrm{~Hz}, 0.3 \mathrm{H}, Z-\mathrm{H}), 4.09$ (q, $J=$ $7.2 \mathrm{~Hz}, 2 \mathrm{H}), 3.12$ (t, $J=7.4 \mathrm{~Hz}, 1 \mathrm{H}), 3.00-2.85(\mathrm{~m}, 2 \mathrm{H}), 2.69$ (q, $J=7.5 \mathrm{~Hz}, 2 \mathrm{H}$ ), $2.40-2.28(\mathrm{~m}, 3 \mathrm{H}), 1.24(\mathrm{t}, J=7.1 \mathrm{~Hz}, 3 \mathrm{H})$.

${ }^{19} \mathrm{~F} \mathrm{NMR}\left(376 \mathrm{MHz}, \mathrm{CDCl}_{3}\right) \delta-105.35(\mathrm{~s}),-105.53(\mathrm{~s})$.

${ }^{13} \mathrm{C}$ NMR $\left(100 \mathrm{MHz}, \mathrm{CDCl}_{3}\right) \delta 197.9,197.8,173.1,173.1,165.7(\mathrm{~d}, J=254.7 \mathrm{~Hz})$, $140.8(\mathrm{~d}, J=230.0 \mathrm{~Hz}), 133.3(\mathrm{~d}, J=2.9 \mathrm{~Hz}), 130.7(\mathrm{~d}, J=9.3 \mathrm{~Hz}), 130.6(\mathrm{~d}, J=9.2$ $\mathrm{Hz}), 128.4,128.3,128.3,128.2,127.0,126.9,126.5,126.2,115.7$ (d, $J=23.1 \mathrm{~Hz})$, $115.6(\mathrm{~d}, J=20.5 \mathrm{~Hz}), 60.4,60.3,38.8,38.4,34.5,33.3,25.1,23.9,23.8,23.2,14.2$, 14.2 (missing two carbons).

HRMS-ESI $(\mathrm{m} / \mathrm{z})[\mathrm{M}+\mathrm{H}]^{+}$calc'd for $\mathrm{C}_{22} \mathrm{H}_{24} \mathrm{FO}_{3}, 335.1704$, found 335.1709. 
Compound 4q ( $\left.{ }^{1} \mathrm{H} \mathrm{NMR}, 400 \mathrm{MHz} \mathrm{CDCl}_{3}\right)$

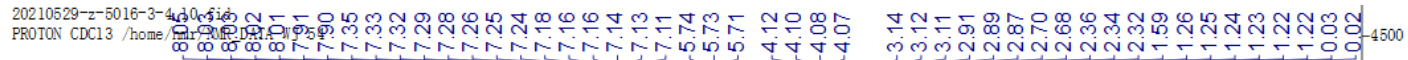

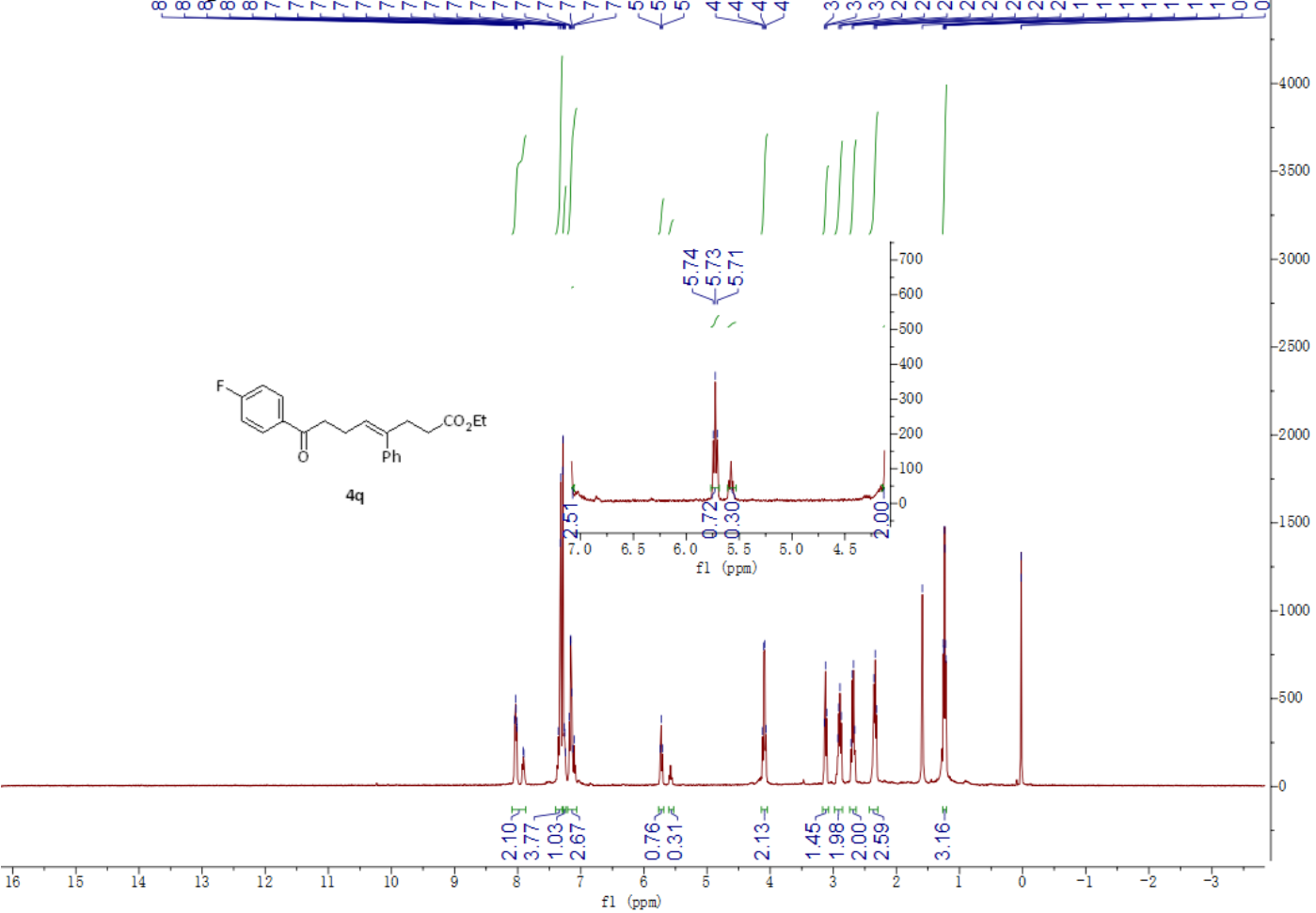

Compound $\mathbf{4 q}\left({ }^{19} \mathrm{~F}\right.$ NMR, $\left.376 \mathrm{MHz}, \mathrm{CDCl}_{3}\right)$

20210529-z-5016-3-4. 11. fid
F19CPD CDC13 /home/nmr/NIR_DATA WJ 54

응

送

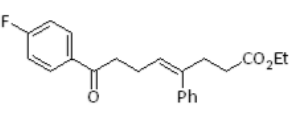

\begin{tabular}{l}
-105.53 \\
\hline \hline$A(s)$ \\
-105.35
\end{tabular}

$4 q$
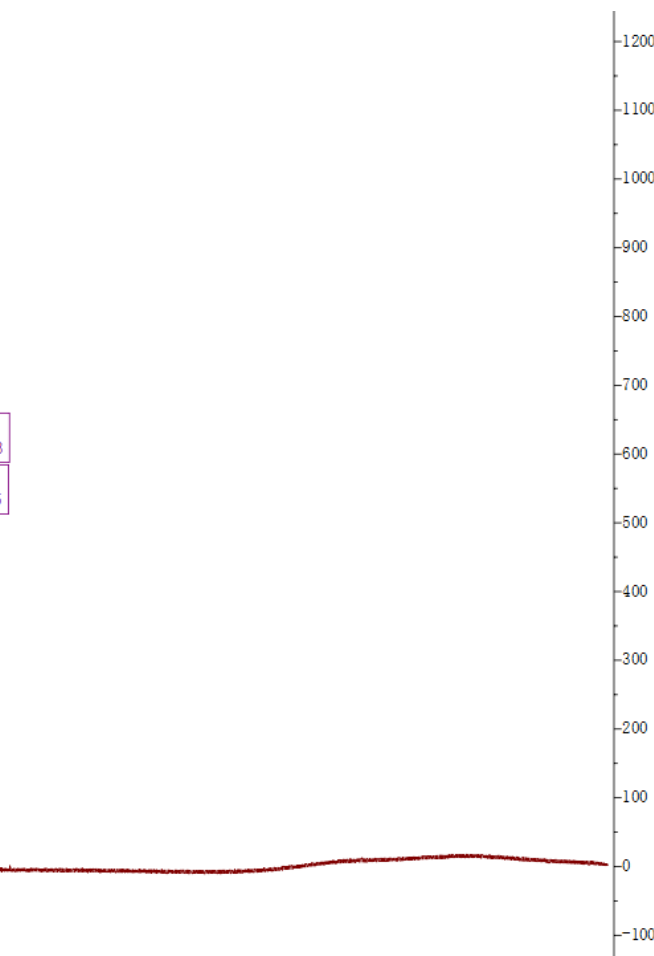

응

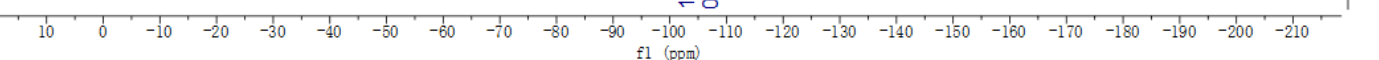

Compound $\mathbf{4 q}\left({ }^{13} \mathrm{C} \mathrm{NMR}, 100 \mathrm{MHz}, \mathrm{CDCl}_{3}\right)$ 


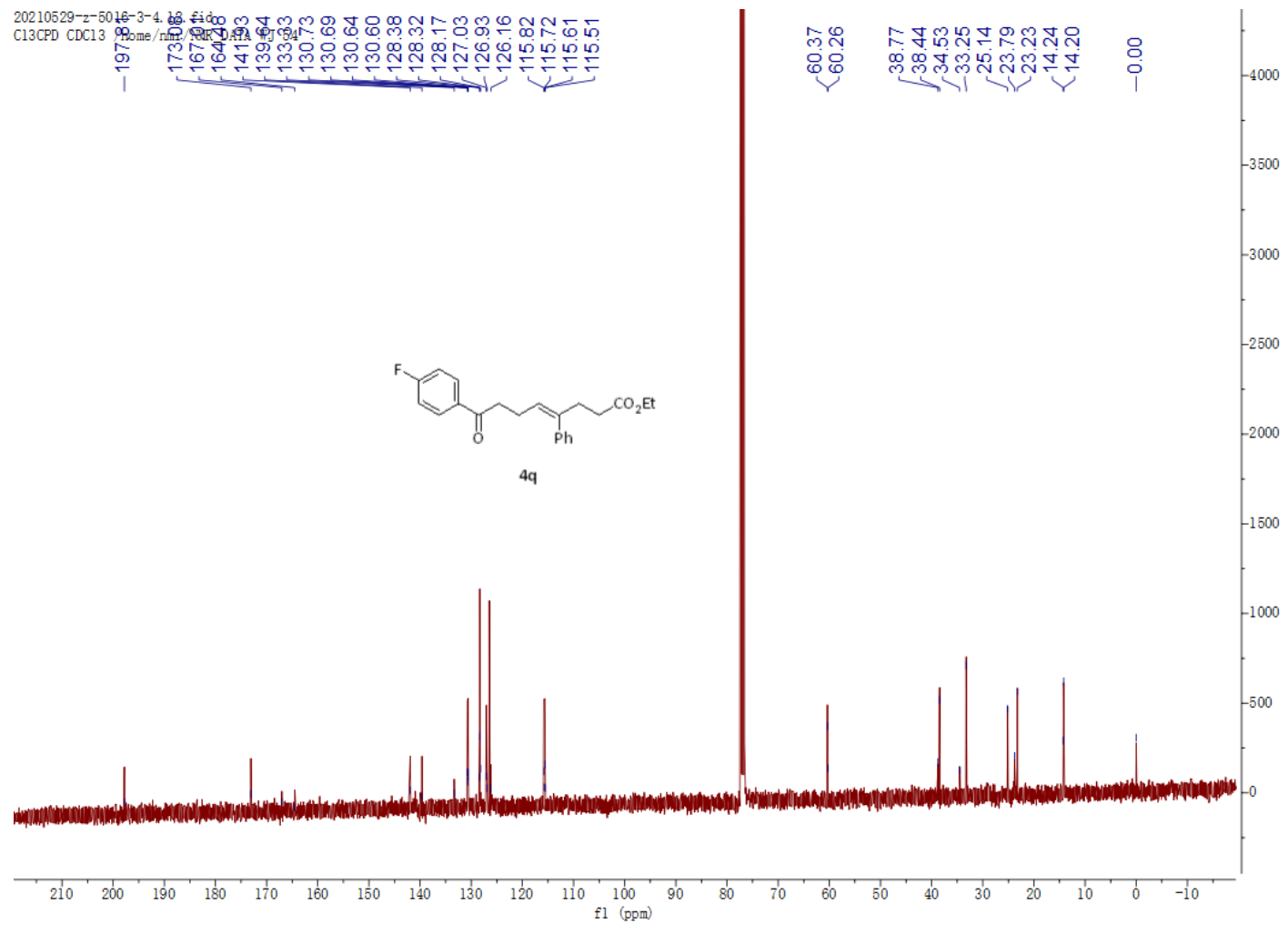

ethyl 3-(3,4-dihydronaphthalen-1-yl)propanoate (7)<smiles>CCC(=O)CCC1=CCCc2ccccc21</smiles>

$\mathrm{PE} / \mathrm{EA}=50 / 1$. Colorless oil, $18.4 \mathrm{mg}, 40 \%$

${ }^{1} \mathrm{H}$ NMR (400 MHz, $\left.\mathrm{CDCl}_{3}\right) \delta 7.41-7.20(\mathrm{~m}, 2 \mathrm{H}), 7.21-7.08(\mathrm{~m}, 2 \mathrm{H}), 5.91(\mathrm{dd}, J=$ 5.3, $3.9 \mathrm{~Hz}, 1 \mathrm{H}), 4.17(\mathrm{q}, J=7.1 \mathrm{~Hz}, 2 \mathrm{H}), 2.87-2.78(\mathrm{~m}, 2 \mathrm{H}), 2.77-2.73(\mathrm{~m}, 2 \mathrm{H})$, 2.58-2.54 (m, 2H), $2.32-2.23(\mathrm{~m}, 2 \mathrm{H}), 1.28(\mathrm{t}, J=7.1 \mathrm{~Hz}, 3 \mathrm{H})$.

${ }^{13} \mathrm{C}$ NMR $\left(100 \mathrm{MHz}, \mathrm{CDCl}_{3}\right) \delta 173.4,136.7,135.0,134.4,127.7,126.8,126.4,125.4$, $122.4,60.4,33.5,28.3,27.9,23.0,14.3$.

HRMS-ESI $(\mathrm{m} / \mathrm{z})[\mathrm{M}+\mathrm{H}]^{+}$calc'd for $\mathrm{C}_{15} \mathrm{H}_{19} \mathrm{O}_{2}, 231.1380$, found 231.1386.

Compound $7\left({ }^{1} \mathrm{H} \mathrm{NMR}, 400 \mathrm{MHz}, \mathrm{CDCl}_{3}\right.$ ) 


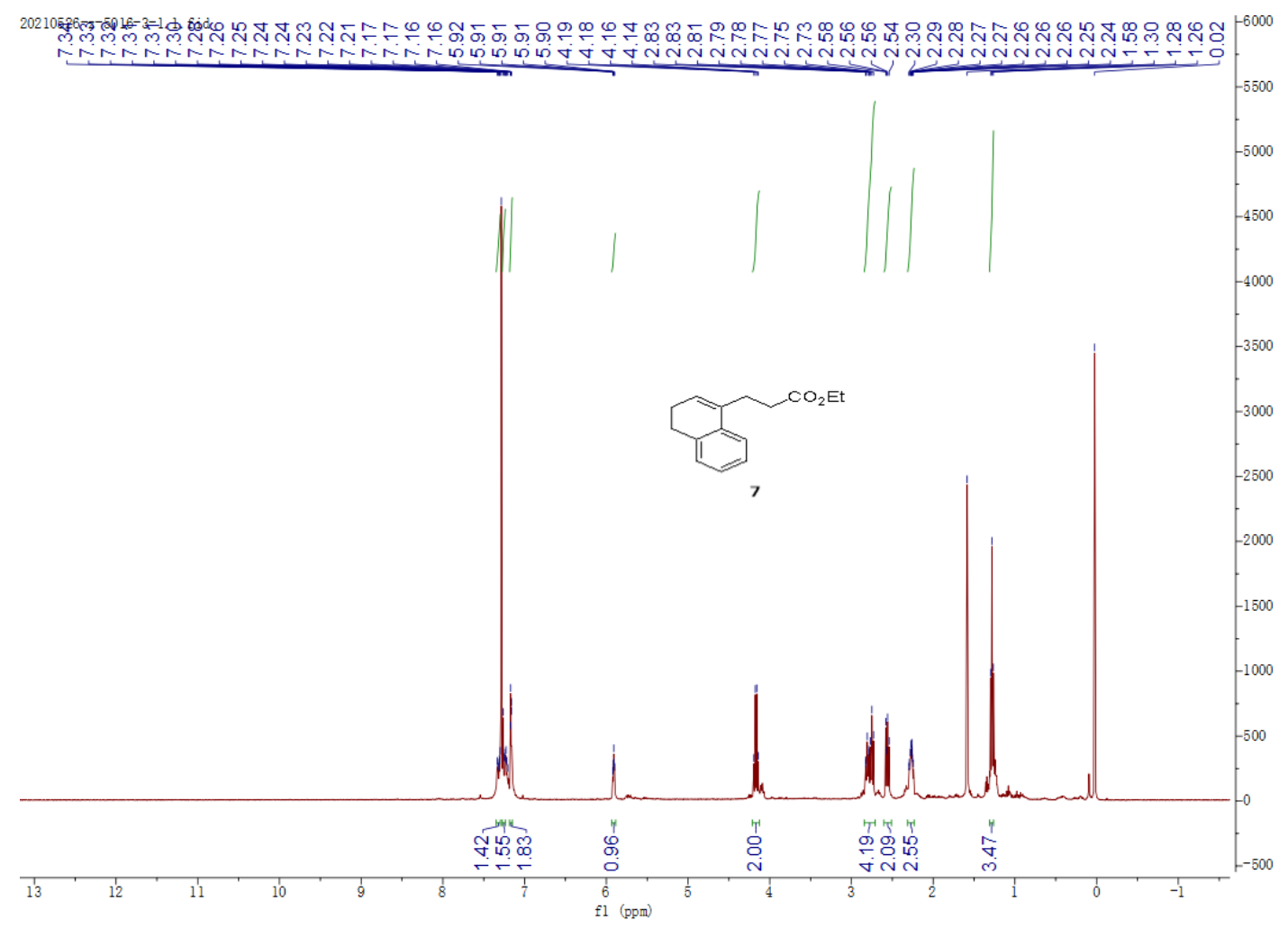

Compound $7\left({ }^{13} \mathrm{C}\right.$ NMR, $\left.100 \mathrm{MHz}, \mathrm{CDCl}_{3}\right)$

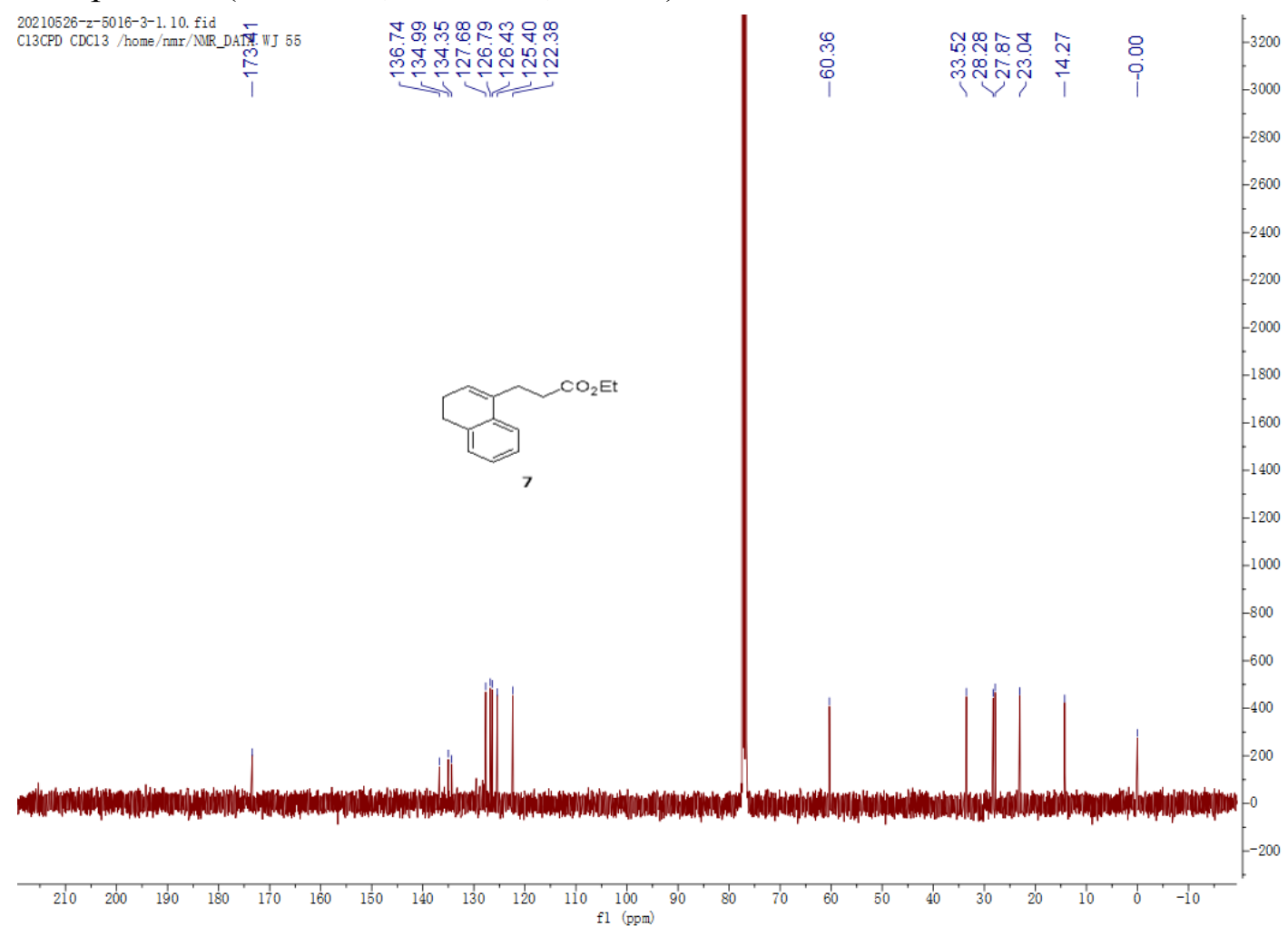

(c) EPR experiment 


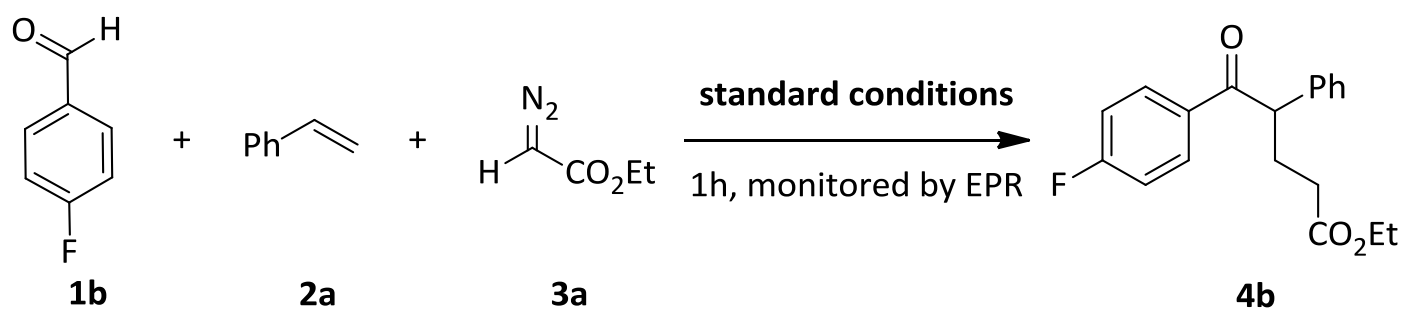

The electron paramagnetic resonance (EPR) measurement of organic radicals was performed on the X-band EPR spectrometer (JEOL, JES FA-200). The spectra were recorded with the microwave power of $0.5-1 \mathrm{~mW}$ at the frequency of $9.0-9.1 \mathrm{GHz}$, modulation frequency of $100 \mathrm{kHz}$, modulation amplitude (or width) of $0.1 \mathrm{mT}$, time constant of $0.1 \mathrm{~s}$, magnetic field sweeping rate of $10 \mathrm{mT} / \mathrm{min}$ and the accumulation times of 4. ESR studies were carried out by using reaction mixture after stirring 10mins. Signals with 3 lines $(\mathrm{g}=2.00393$, linewidth $=0.338 \mathrm{mT}$, hyperfine coupling constant $\mathrm{a}_{\mathrm{N}}=13.23 \mathrm{MHz}, \mathrm{a}_{\mathrm{H}}=4.67 \mathrm{MHz}$ ) were observed and identified as EPR signals of Int. II, which are in good agreement with literature ${ }^{3}$ (Figure S3). Signals with 9 lines were a mixed spectrum which could be seperated to a signal with 3 lines (Int. II) and a signal with 6 lines $(\mathrm{g}=2.00429$, linewidth $=0.196 \mathrm{mT}$, hyperfine coupling constant $\mathrm{a}_{\mathrm{N}}=40.99 \mathrm{MHz}, \mathrm{a}_{\mathrm{H}}=59.90 \mathrm{MHz}$ ) which was identified as EPR signals of radical $\mathbf{c}$ in good agreement with literature ${ }^{4}$.

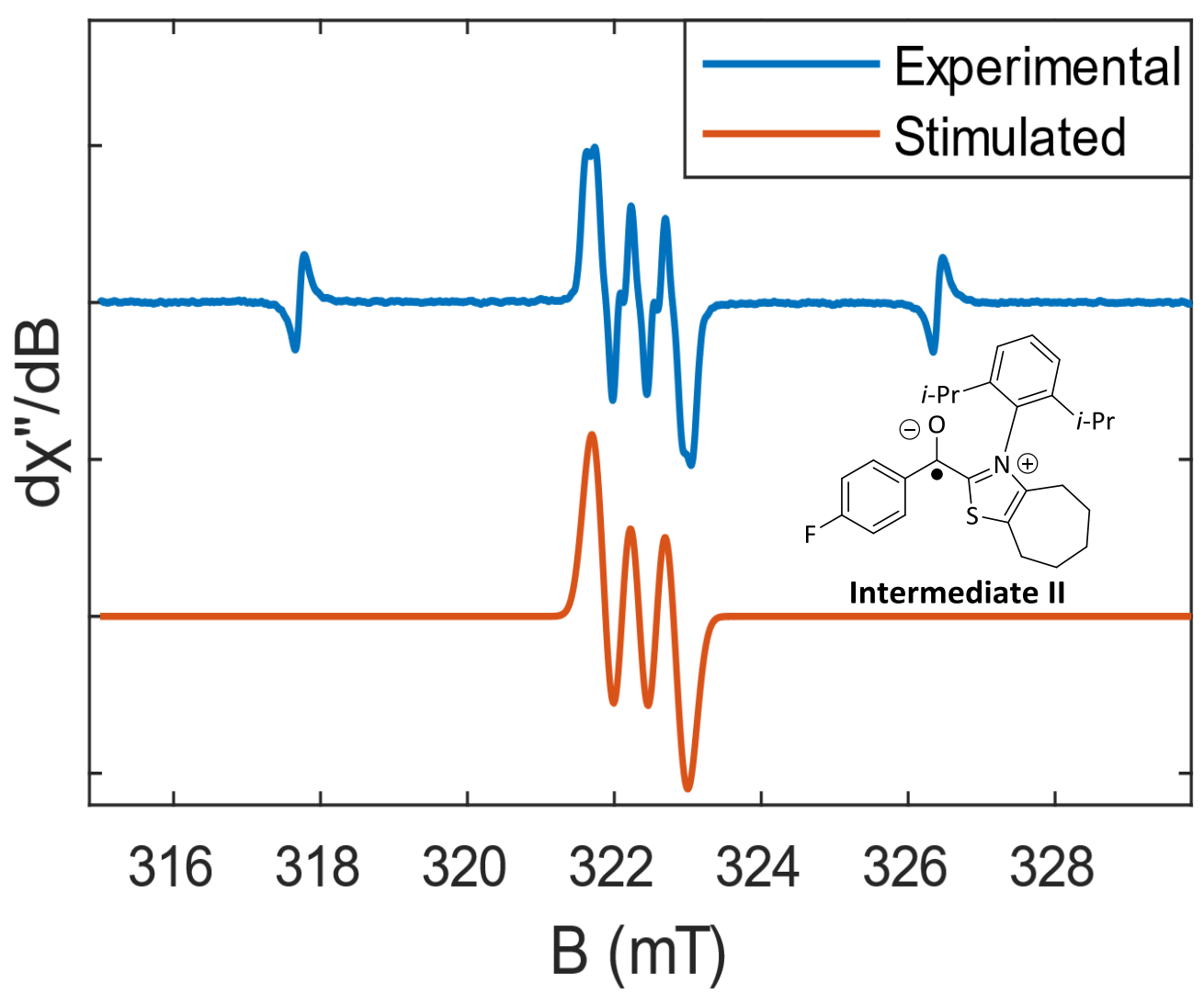

Fig S3. (Blue line) EPR spectra of radical derived from trapping intermediate II in DMSO according to standard conditions in general procedure. (Red line) Stimulated spectra, $g=2.00393$, linewidth $=0.338 \mathrm{mT}$, hyperfine coupling constant $\mathrm{a}_{\mathrm{N}}=13.23$ $\mathrm{MHz}, \mathrm{a}_{\mathrm{H}}=4.67 \mathrm{MHz}$. Root mean square error (RMSD) of this stimulation is 0.06 . 


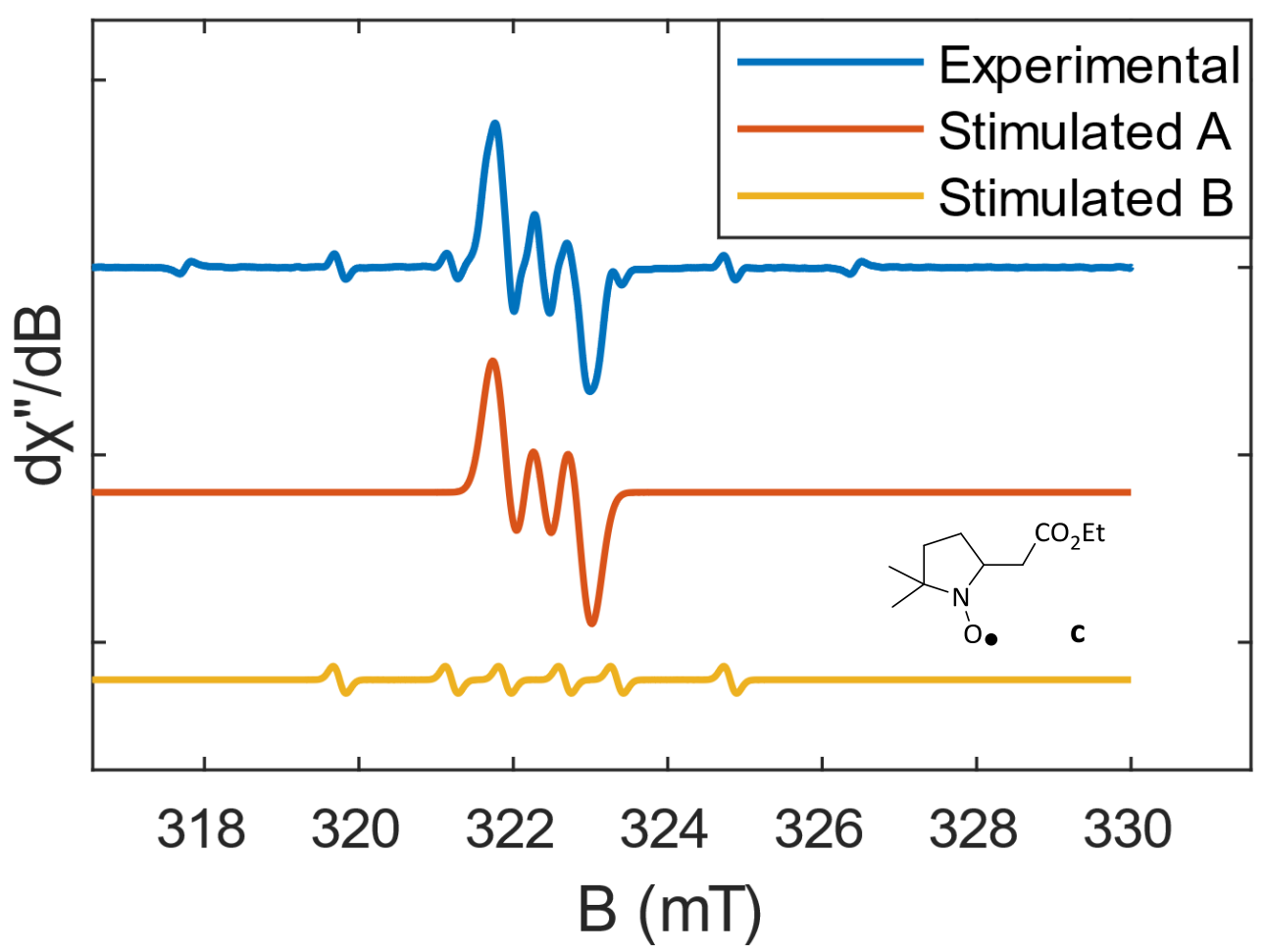

Fig S4. (Blue line) EPR spectra of radical derived from trapping intermediate II and c with DMPO in DMSO according to standard conditions in general procedure. (Red line) stimulated spectra for intermediate II, $g=2.00374$, linewidth $=0.354 \mathrm{mT}$, hyperfine coupling constant $\mathrm{a}_{\mathrm{N}}=12.66 \mathrm{MHz}, \mathrm{a}_{\mathrm{H}}=4.89 \mathrm{MHz}$. Stimulated spectra for $\mathbf{c}$, $\mathrm{g}=2.00429$, linewidth $=0.196 \mathrm{mT}$, hyperfine coupling constant $\mathrm{a}_{\mathrm{N}}=40.99 \mathrm{MHz}, \mathrm{a}_{\mathrm{H}}=$ $59.90 \mathrm{MHz}$

Root mean square error (RMSD) of this stimulation is 0.026 .

(d) Isotope-labeling experiment 
<smiles>[2H]C(=O)c1ccccc1</smiles>

$1 a^{\prime}$

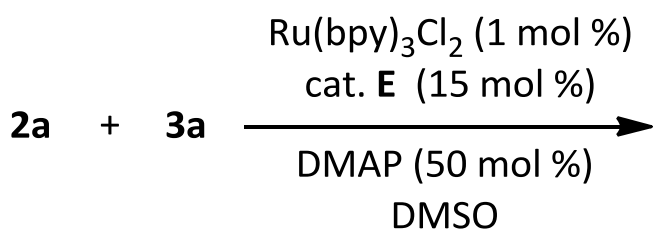

blue LED<smiles>CCOC(=O)C(CCO)CC(C(=O)c1ccccc1)c1ccccc1</smiles>

4aa (41\%)

(a)

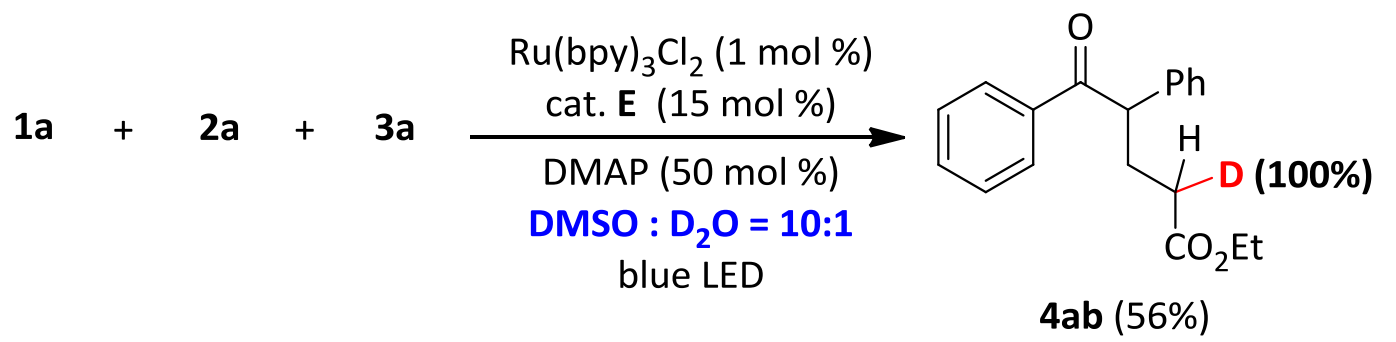

(b)

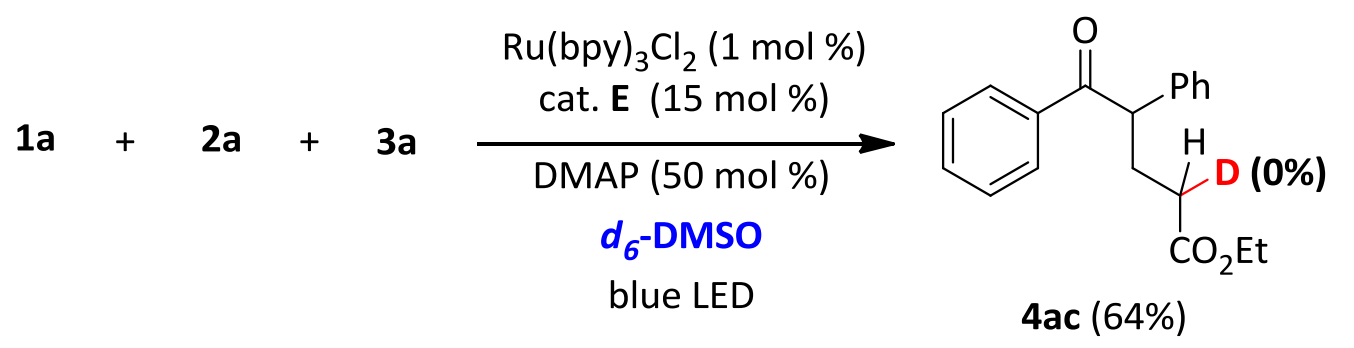

(c)

Fig. S5 Isotope-labeling study.

Reaction 1: Prepared according to the General Procedure using 1a-D $\mathbf{D}_{\mathbf{8}}$ (25 mg, 1.2 equiv, $0.24 \mathrm{mmol}$ ) instead of 1a. ${ }^{1} \mathrm{H}$ NMR analysis showed $10 \%$ deuterium incorporation.

Reaction 2: Prepared according to the General Procedure adding $\mathbf{D}_{\mathbf{2}} \mathbf{O}(100 \mathrm{uL})$ as additive. ${ }^{1} \mathrm{H}$ NMR analysis showed $100 \%$ deuterium incorporation.

Reaction 3: Prepared according to the General Procedure using $\boldsymbol{d}_{\boldsymbol{6}^{-}}$-DMSO (2 mL) instead of DMSO. ${ }^{1} \mathrm{H}$ NMR analysis showed $0 \%$ deuterium incorporation.

Compound 4aa $\left({ }^{1} \mathrm{H} \mathrm{NMR}, 400 \mathrm{MHz}, \mathrm{CDCl}_{3}\right)$ 


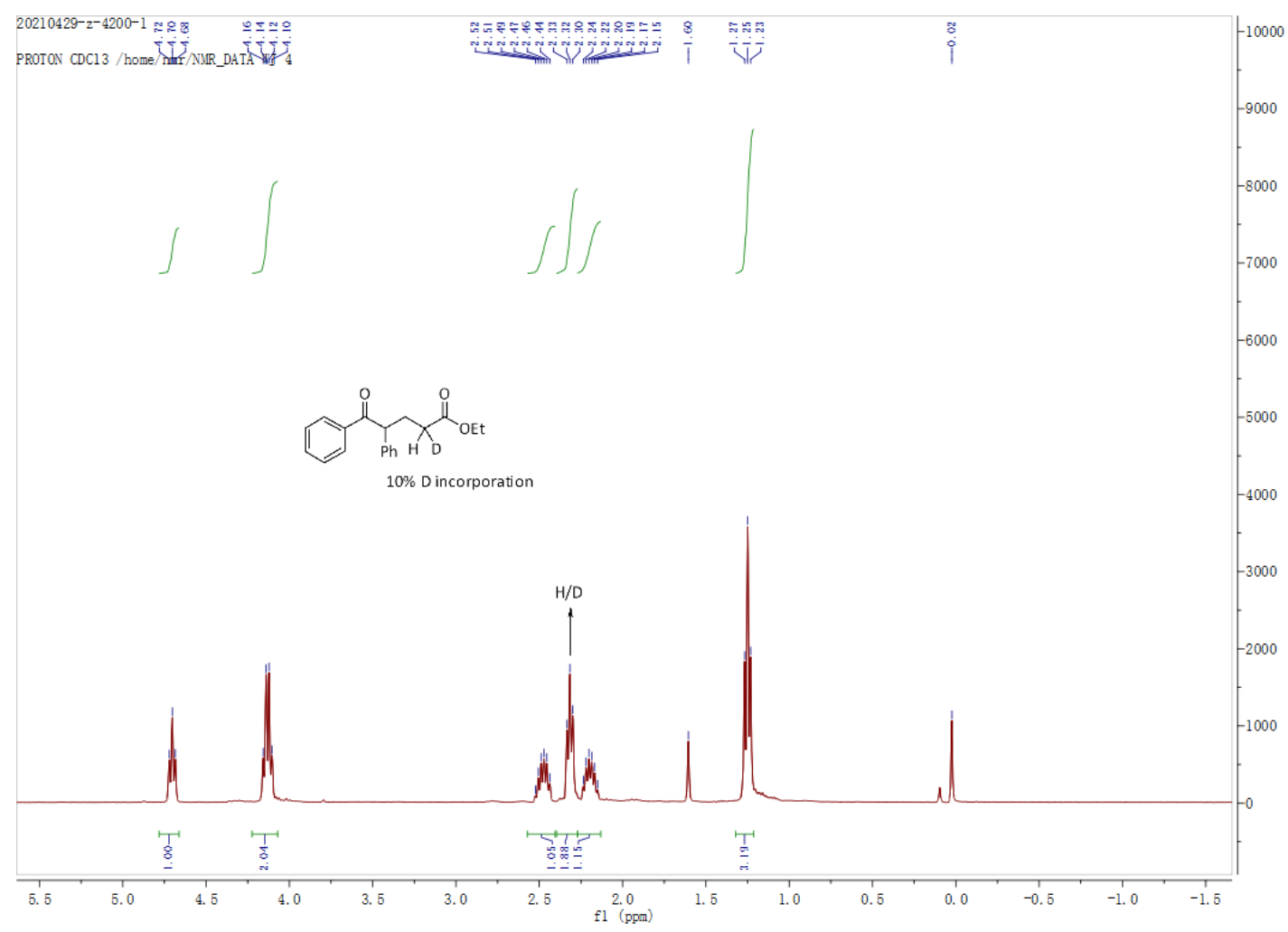

Compound 4ab $\left({ }^{1} \mathrm{H} \mathrm{NMR}, 400 \mathrm{MHz}, \mathrm{CDCl}_{3}\right)$

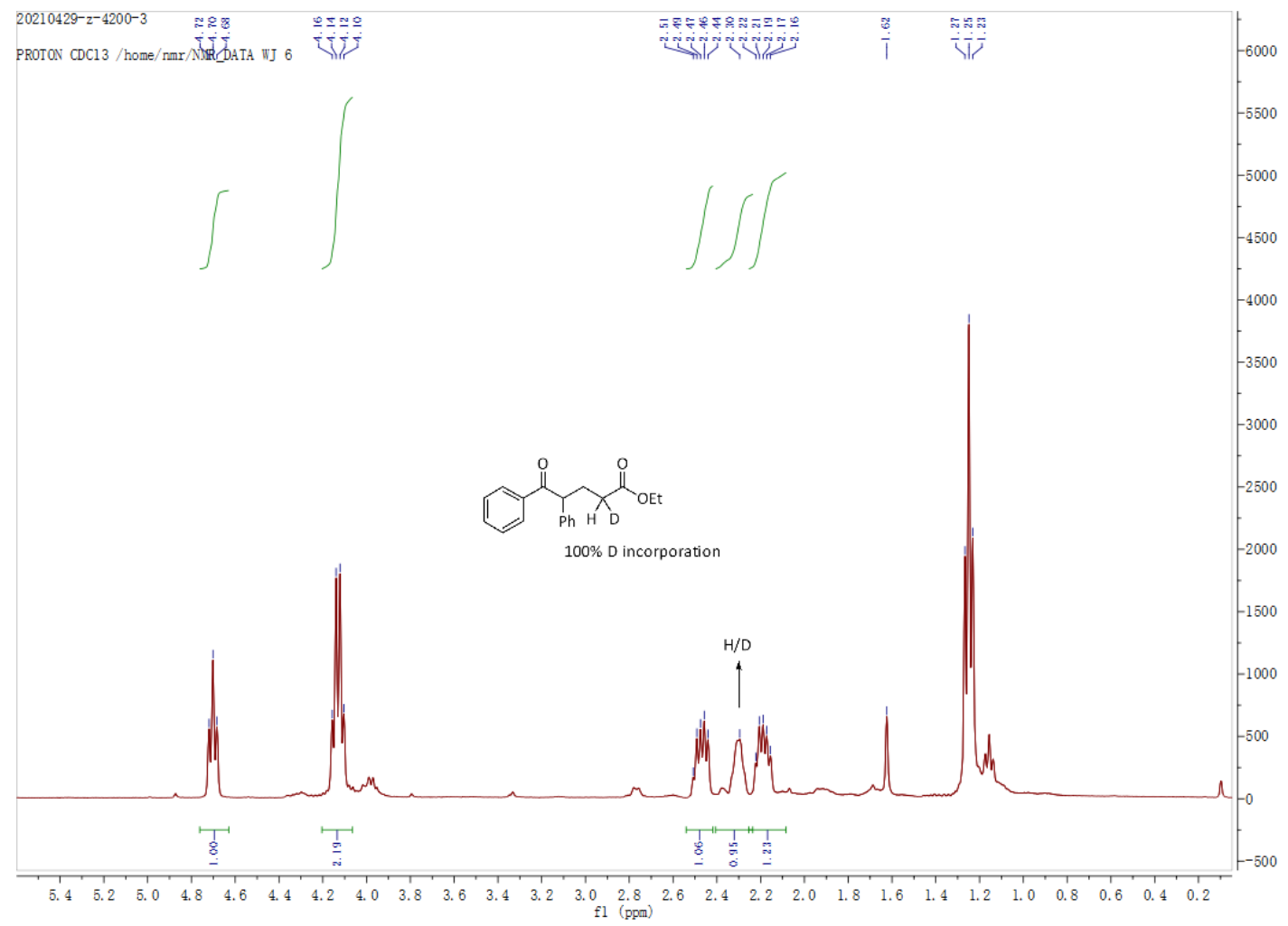

Compound 4ac ( ${ }^{1} \mathrm{H}$ NMR, $\left.400 \mathrm{MHz}, \mathrm{CDCl}_{3}\right)$ 


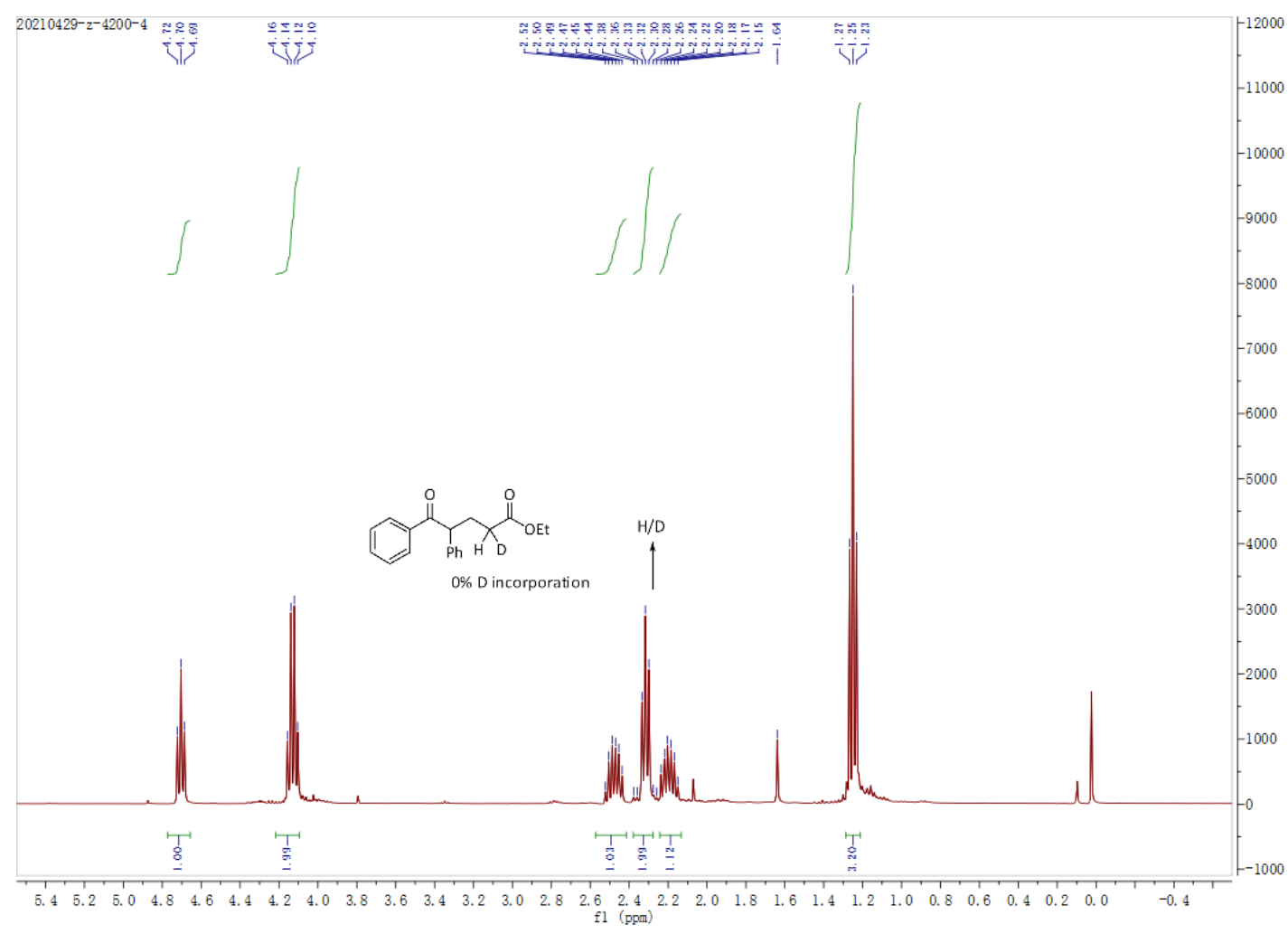

(e) Control experiment

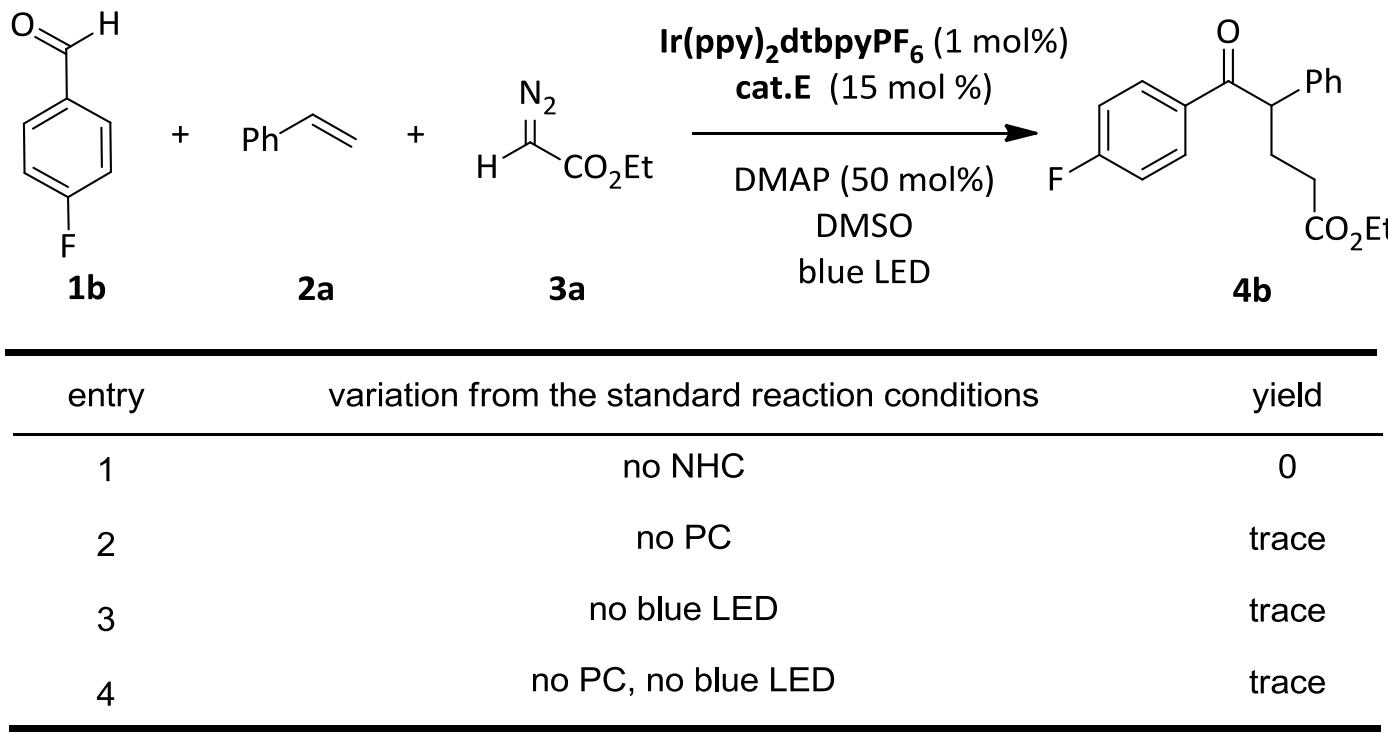

$\mathrm{NHC}$ catalyst, $\operatorname{Ir}(\mathrm{ppy})_{2} \mathrm{dtbpyPF}_{6}$ and blue light were all requisite for this reaction.

(f) Light-on-off experiment 


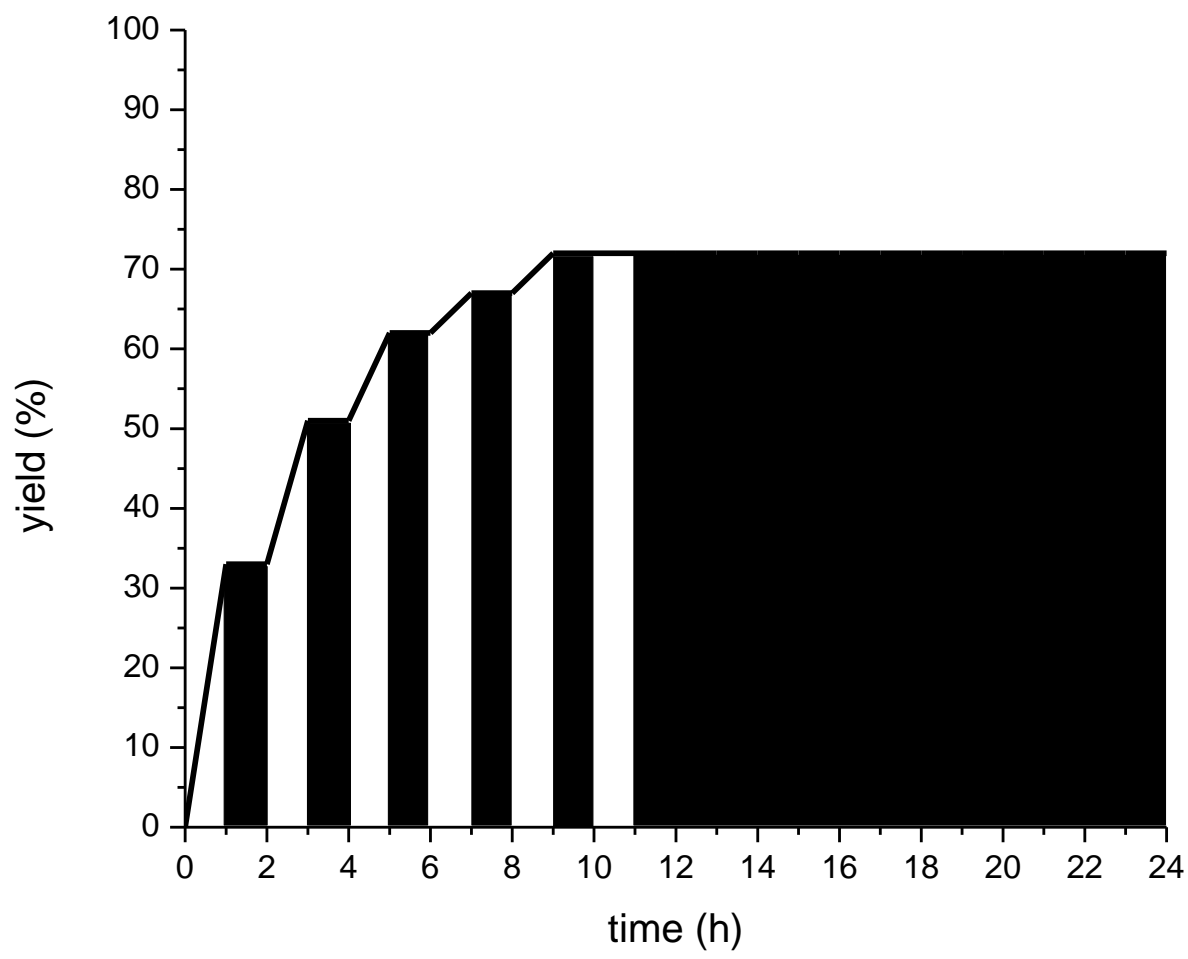

Fig.S6 Light on off experiment

According to the standard procedure, precatalyst $\mathbf{E}(12 \mathrm{mg}, 0.03 \mathrm{mmol}, 0.15$ equiv), DMAP (12 mg, $0.22 \mathrm{mmol}, 0.5$ equiv), $\operatorname{Ir}(\mathrm{ppy})_{2} \mathrm{dtbpyPF}_{6}(2.0 \mathrm{mg}, 0.002 \mathrm{mmol}, 0.01$ equiv) and DMSO ( $2 \mathrm{~mL}, 0.1 \mathrm{M})$ were added first. 1a ( $0.24 \mathrm{mmol}, 1.2$ equiv), $2 \mathbf{a}(0.2$ mmol, 1.0 equiv), $3 \mathbf{a}$ ( $0.4 \mathrm{mmol}, 0.2$ equiv) were added subsequently. Then, the tube was caped. The resulting solution was irradiated by a $30 \mathrm{~W}$ blue LED $(450 \mathrm{~nm})$ with stirring at a distance of $3 \mathrm{~cm}$ (with cooling by the fan) at $25{ }^{\circ} \mathrm{C}$ for about $24 \mathrm{~h}$. Yield was determined by GC-MS of the crude mixture using 1,3,5- trimethoxybenzene (33.6 $\mathrm{mg}, 0.20 \mathrm{mmol}, 1.0$ equiv) as internal standard.

(g) Luminesence quenching experiment 


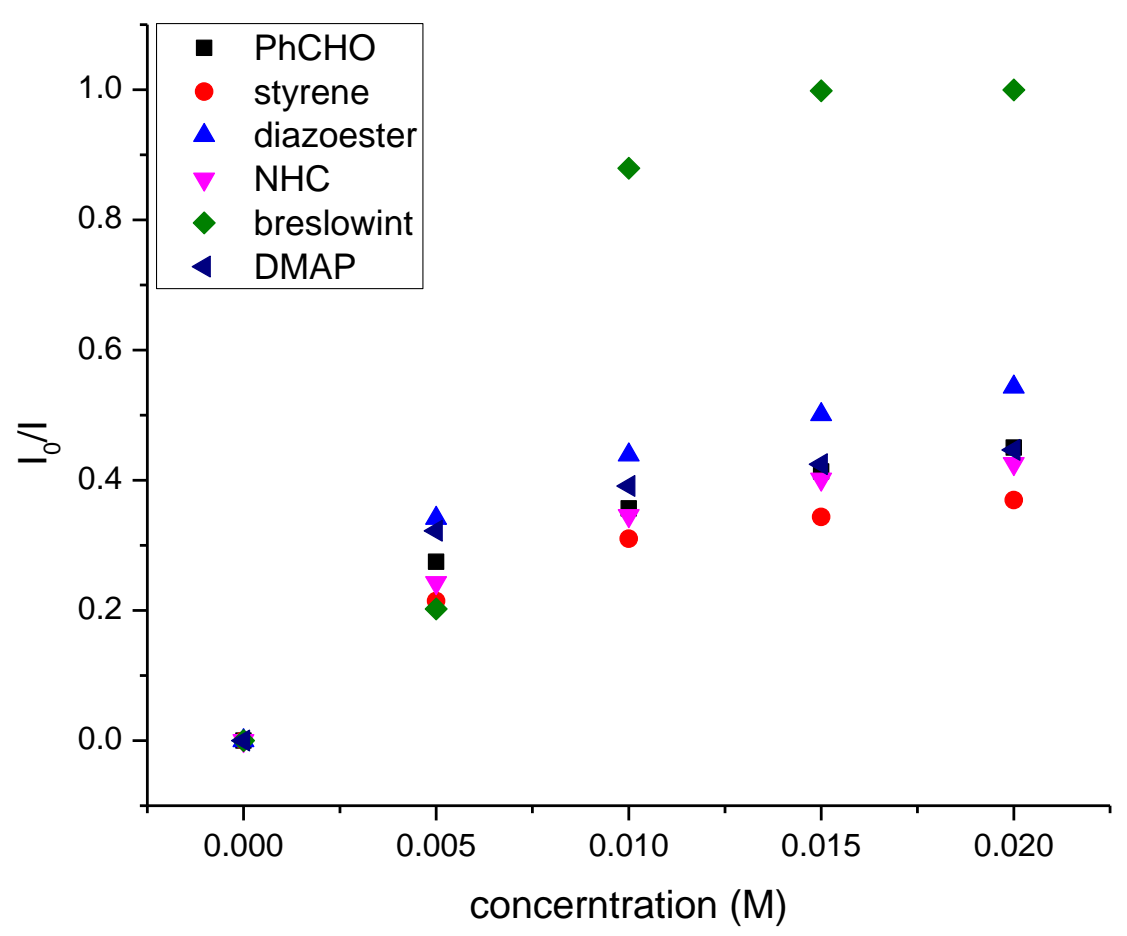

Fig.S7 Luminescence quenching experiment

Emission intensities results were monitored by UV-800C Fluorescence Spectrometer (Tsinghua instrument) for all experiments. All $\operatorname{Ir}(\mathrm{ppy})_{2} \mathrm{dtbpyPF} \mathrm{P}_{6}$ solutions were excited at $410 \mathrm{~nm}$ and the emission intensity was collected at 400-850 $\mathrm{nm}$. In general, the DMSO as solution of $\operatorname{Ir}(\mathrm{ppy})_{2} \mathrm{dtbpyPF}_{6}(10 \mu \mathrm{M})$ was added the relative quencher in a $4.5 \mathrm{~cm}$ quartz cuvette. After stirring for $5 \mathrm{~min}$, the emission spectra were collected. The results showed that the Breslow Intermediate could obviously quench the photoexcited $\operatorname{Ir}(\mathrm{ppy})_{2} \mathrm{dtbpyPF}_{6}$, while others like $\mathrm{PhCHO}$, styrene, diazo ester, DMAP, and NHC-E were less effective.

The emission intensity at $595 \mathrm{~nm}$ was recorded. And the decreasing trend of emission intensities depends on the concentration of the mixture (Breslow intermediate). 


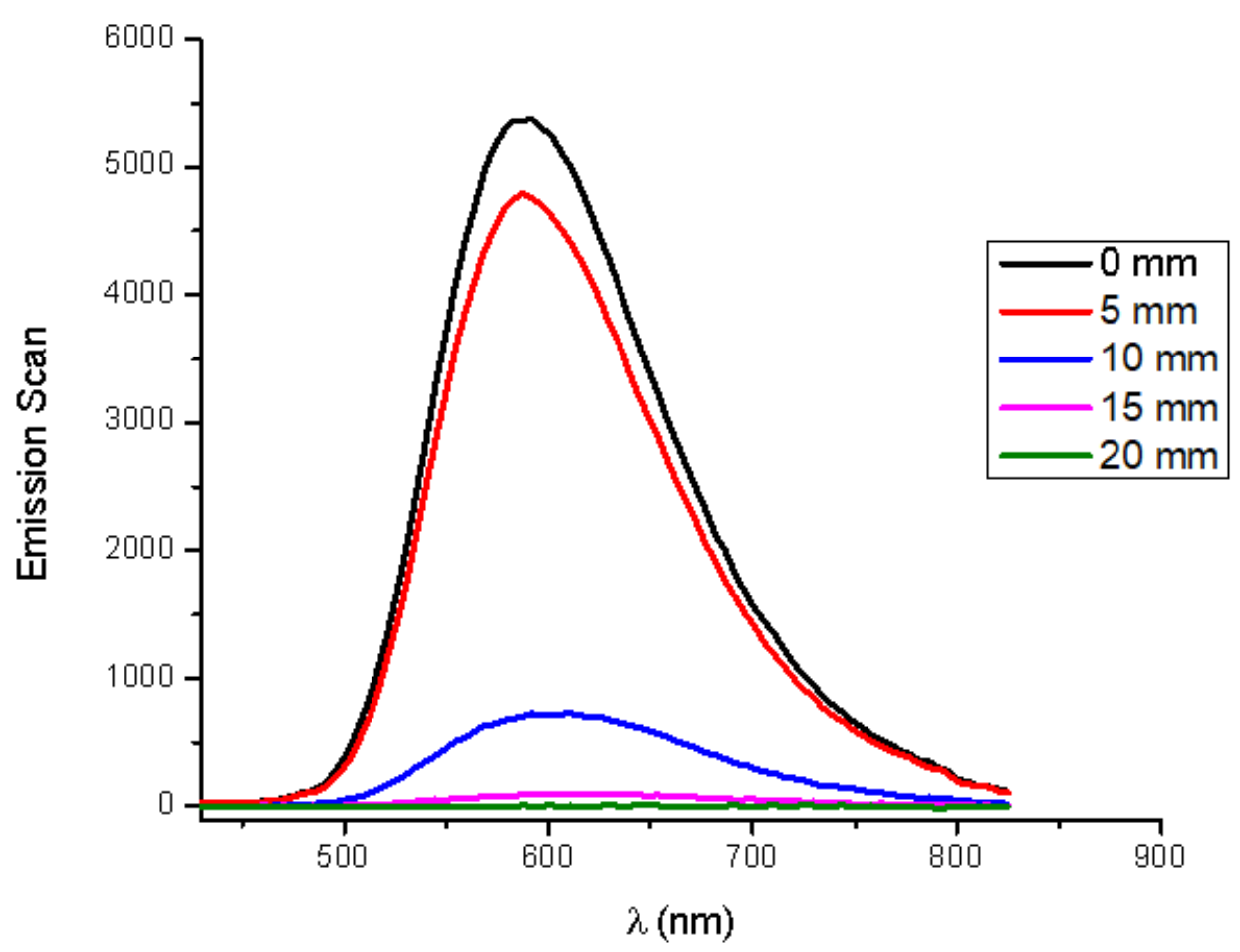

Fig S8. Luminescence quenching results of Breslow intermediate.

\section{1 mmol Scale-up Experiment}

In a glovebox, to a flame-dried $50 \mathrm{~mL}$ Schlenk reaction tube equipped with a magnetic stir bar, was added the precatalyst $\mathbf{E}(62.1 \mathrm{mg}, 0.15 \mathrm{mmol}, 0.15$ equiv), DMAP (61.0 mg, 0.5 mmol, 0.5 equiv), Ir(ppy) ${ }_{2} \mathrm{dtbpyPF}_{6}(9.14 \mathrm{mg}, 0.01 \mathrm{mmol}, 0.01$ equiv) and DMSO (10 mL, 0.1 M). 1a (148.8 mg, $1.2 \mathrm{mmol}, 1.2$ equiv), 2 a (106.0 mg, $1 \mathrm{mmol}, 1.0$ equiv), $3 \mathbf{a}$ ( $228.0 \mathrm{mg}, 2 \mathrm{mmol}, 2.0$ equiv) were added subsequently. Then, the tube was caped and taken out of glovebox. The resulting solution was irradiated by a $30 \mathrm{~W}$ blue LED $(450 \mathrm{~nm}$ ) with stirring at a distance of $3 \mathrm{~cm}$ (with cooling by the fan) at $25{ }^{\circ} \mathrm{C}$ for about $8 \mathrm{~h}$ until the styrene was consumed completely, as monitored by TLC analysis. The mixture was diluted with EtOAc, washed with water and dried by $\mathrm{Na}_{2} \mathrm{SO}_{4}$. The organic phase was then concentrated under reduced pressure and purified by column chromatography on silica gel (Petroleum Ether/EtOAc, $30: 1$ ) to afford the desired product $\mathbf{4 b}$ as a colorless oil, $254.3 \mathrm{mg}, 81 \%$ yield. 


\section{References}

1. Tang, M.; Han, S.; Huang, S.; Huang, S.; Xie, L.-G. Carbosulfenylation of Alkenes with Organozinc Reagents and Dimethyl(Methylthio)Sulfonium Trifluoromethanesulfonate. Org. Lett. 2020, 22, 9729-9734.

2. Cong, F.; Wei, Y.; Tang, P. Combining Photoredox and Silver Catalysis for Azidotrifluoromethoxylation of Styrenes. Chem. Commun. 2018, 54, 4473-4476.

3. Gockel, S. N.; Lee, S.; Gay, B. L.; Hull, K. L. Oxidative Three-Component Carboamination of Vinylarenes with Alkylboronic Acids. ACS Catal. 2021, 11, 5166-5171.

4. Dong, X.-Y.; Cheng, J.-T.; Zhang, Y.-F.; Li, Z.-L.; Zhan, T.-Y.; Chen, J.-J.; Wang, F.-L.; Yang, N.-Y.; Ye, L.; Gu, Q.-S.; Liu, X.-Y. Copper-Catalyzed Asymmetric Radical 1,2-Carboalkynylation of Alkenes with Alkyl Halides and Terminal Alkynes. J. Am. Chem. Soc. 2020, 142, 9501-9509.

5. Hari, D. P.; Waser, J. Enantioselective Copper-Catalyzed Oxy-Alkynylation of Diazo Compounds. J. Am. Chem. Soc. 2017, 139, 8420-8423.

6. (6) Doyle, M. P.; Dyatkin, A. B. Spirolactones from Dirhodium(Ii)-Catalyzed Diazo Decomposition with Regioselective Carbon-Hydrogen Insertion. J. Org. Chem. 1995, 60, 3035-3038.

7. Neuhaus, J. D.; Bauer, A.; Pinto, A.; Maulide, N. A Catalytic Cross-Olefination of Diazo Compounds with Sulfoxonium Ylides. Angew. Chem. Int. Ed. 2018, 57, 16215-16218.

\section{NMR Spectra}

Compound 4a

${ }^{1} \mathrm{H} \mathrm{NMR}\left(\mathrm{CDCl}_{3}, 400 \mathrm{MHz}\right)$ 


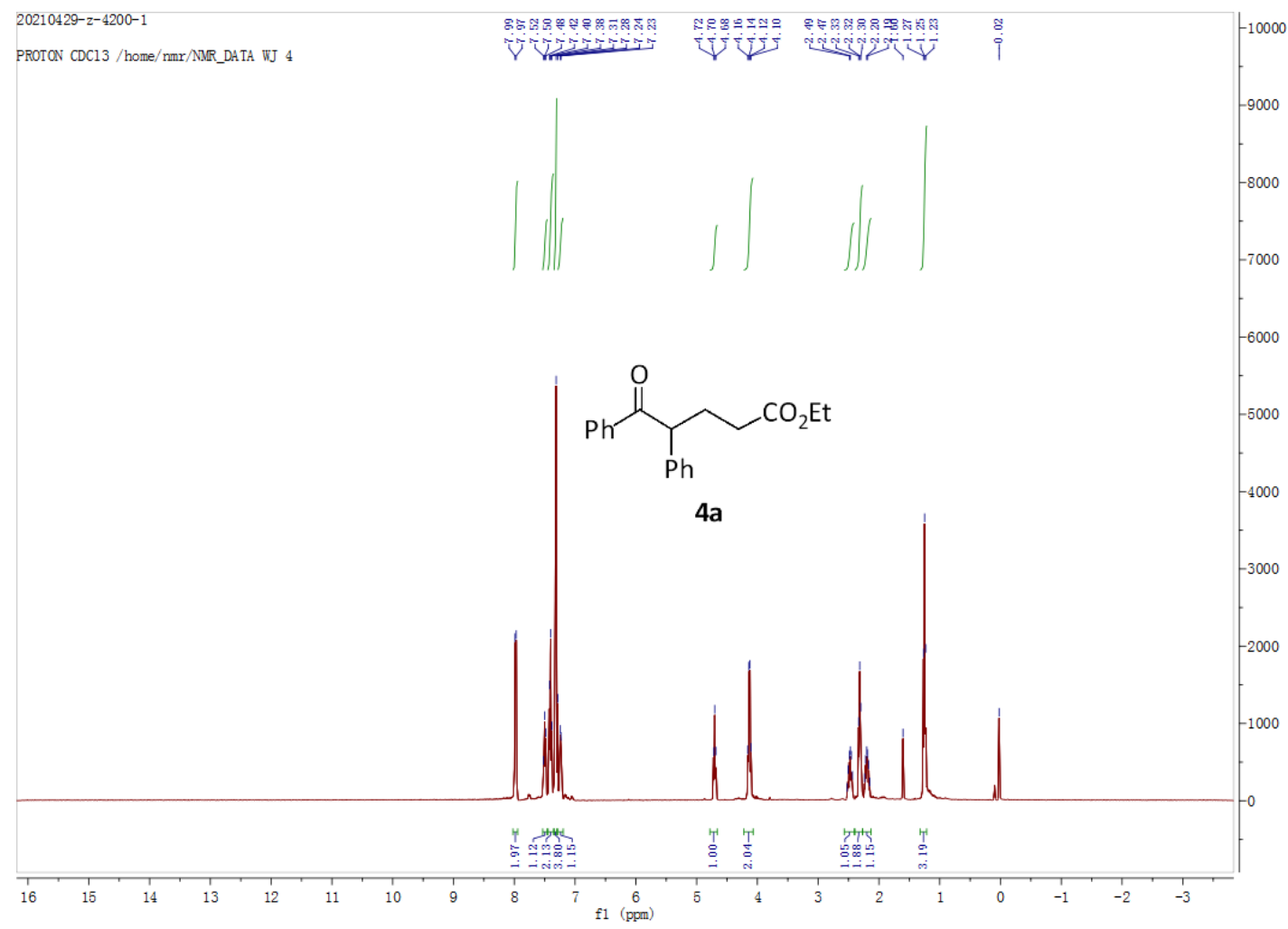

${ }^{13} \mathrm{C} \mathrm{NMR}\left(\mathrm{CDCl}_{3}, 100 \mathrm{MHz}\right)$

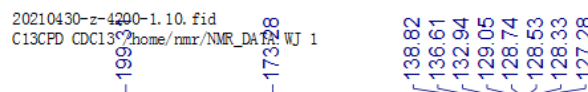

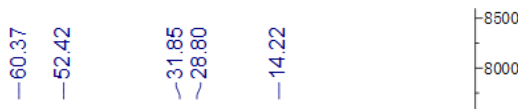

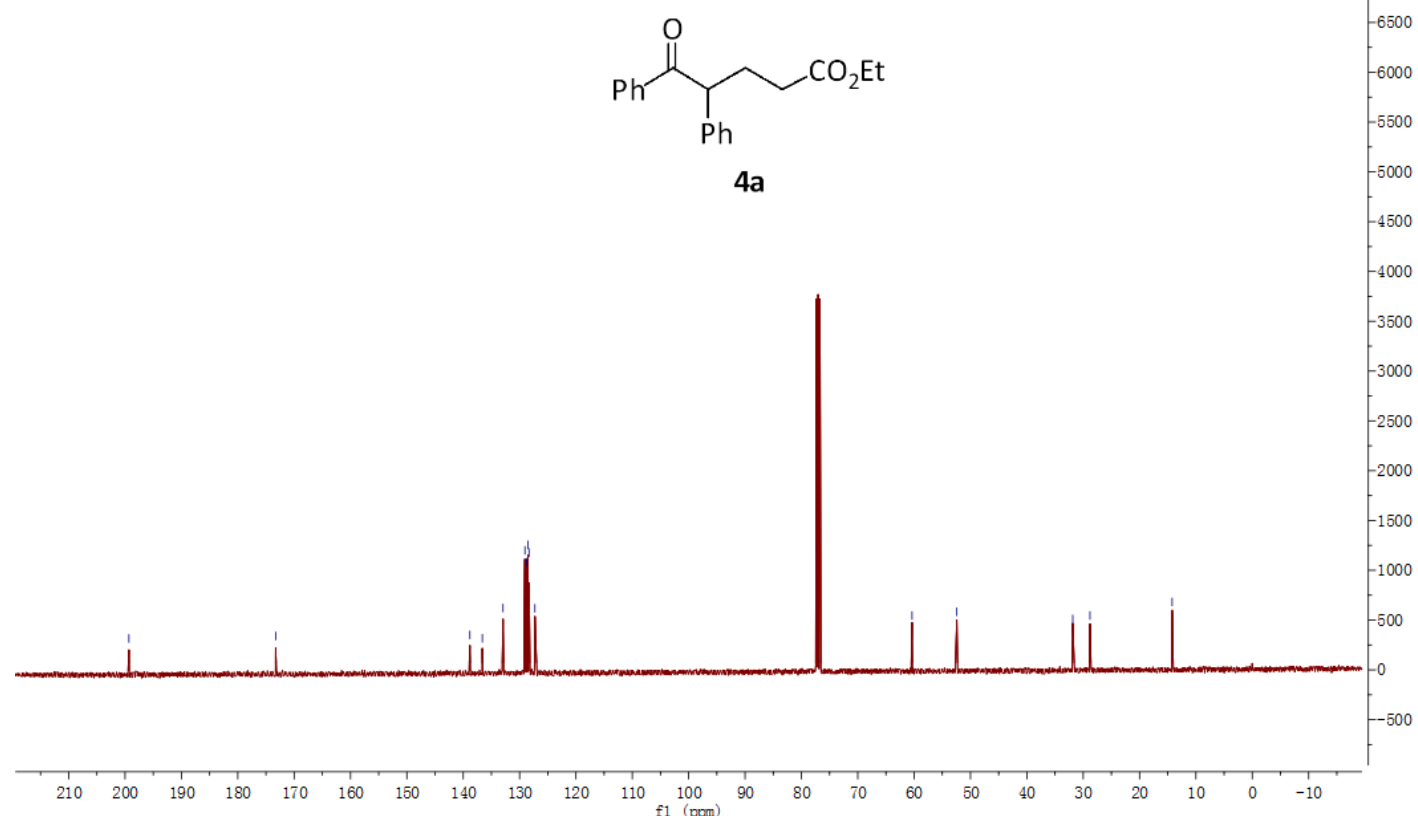

$4 a$ 


\section{Compound $\mathbf{4 b}$}

${ }^{1} \mathrm{H} \mathrm{NMR}\left(\mathrm{CDCl}_{3}, 400 \mathrm{MHz}\right)$

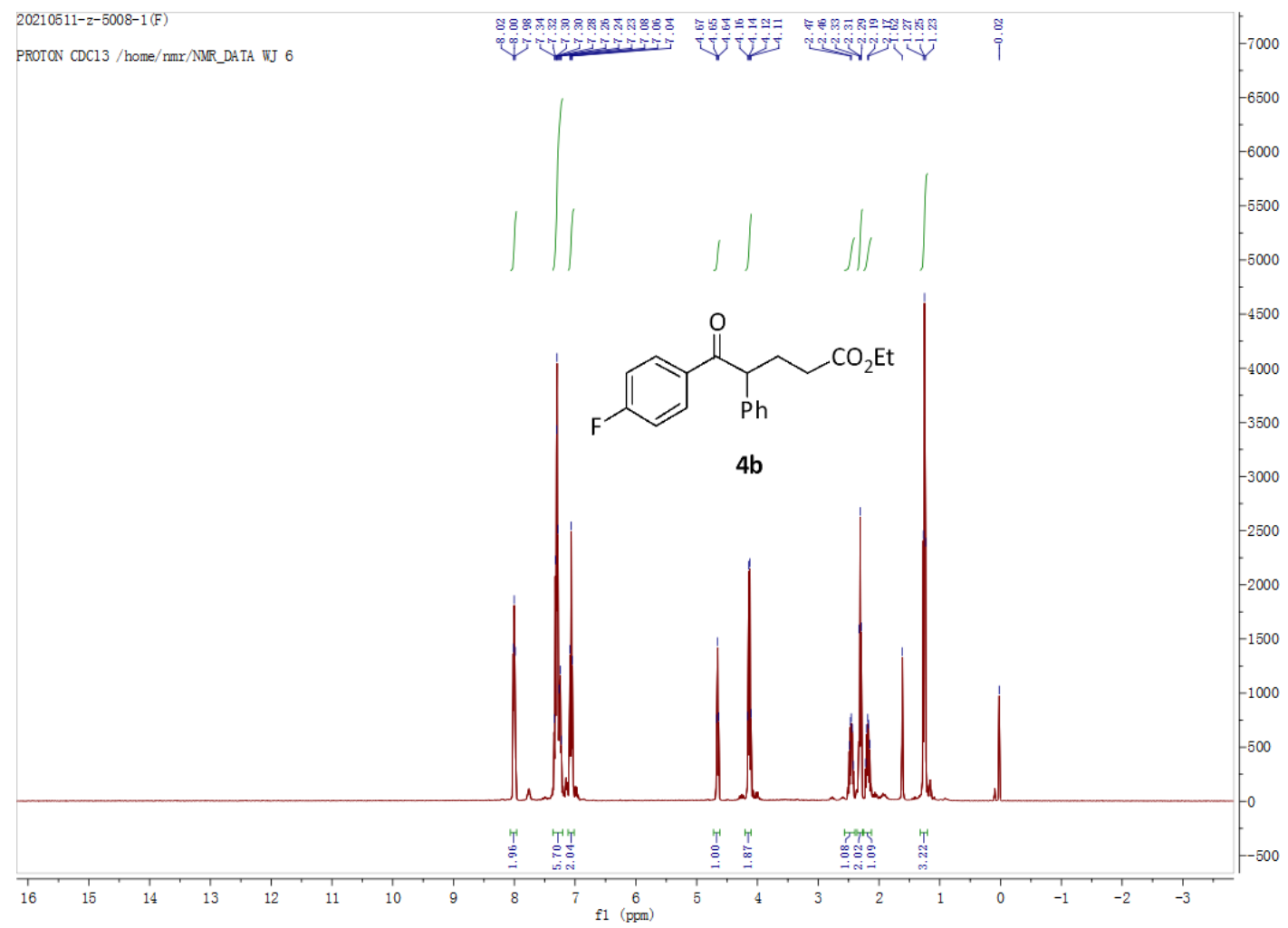

${ }^{19} \mathrm{~F} \mathrm{NMR}\left(\mathrm{CDCl}_{3}, 376 \mathrm{MHz}\right)$

20210511-z-5008-1(F)

F19CPD CDC13 /home/nmr/NMR_DATA WJ 6

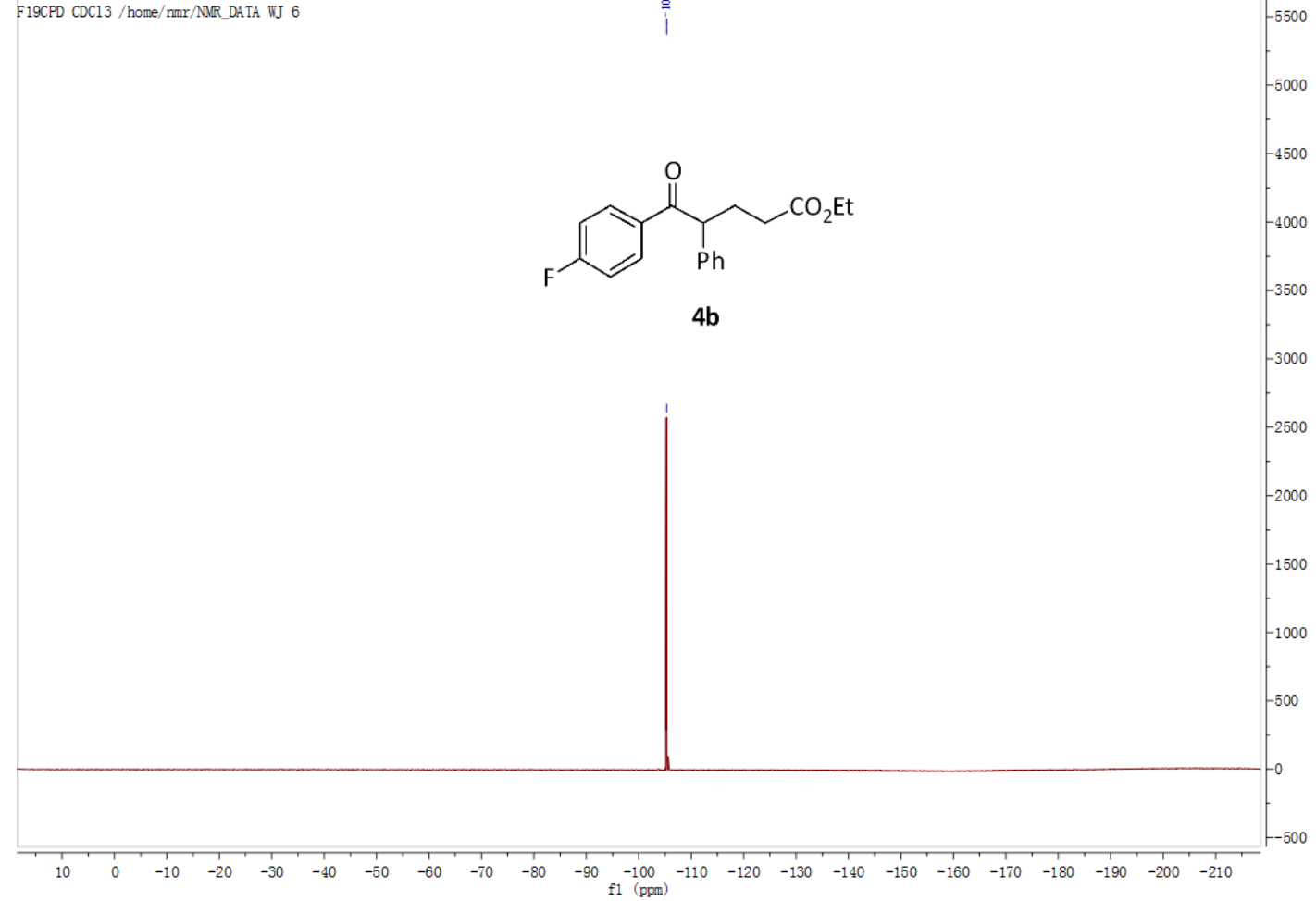

${ }^{13} \mathrm{C} \mathrm{NMR}\left(\mathrm{CDCl}_{3}, 100 \mathrm{MHz}\right)$ 


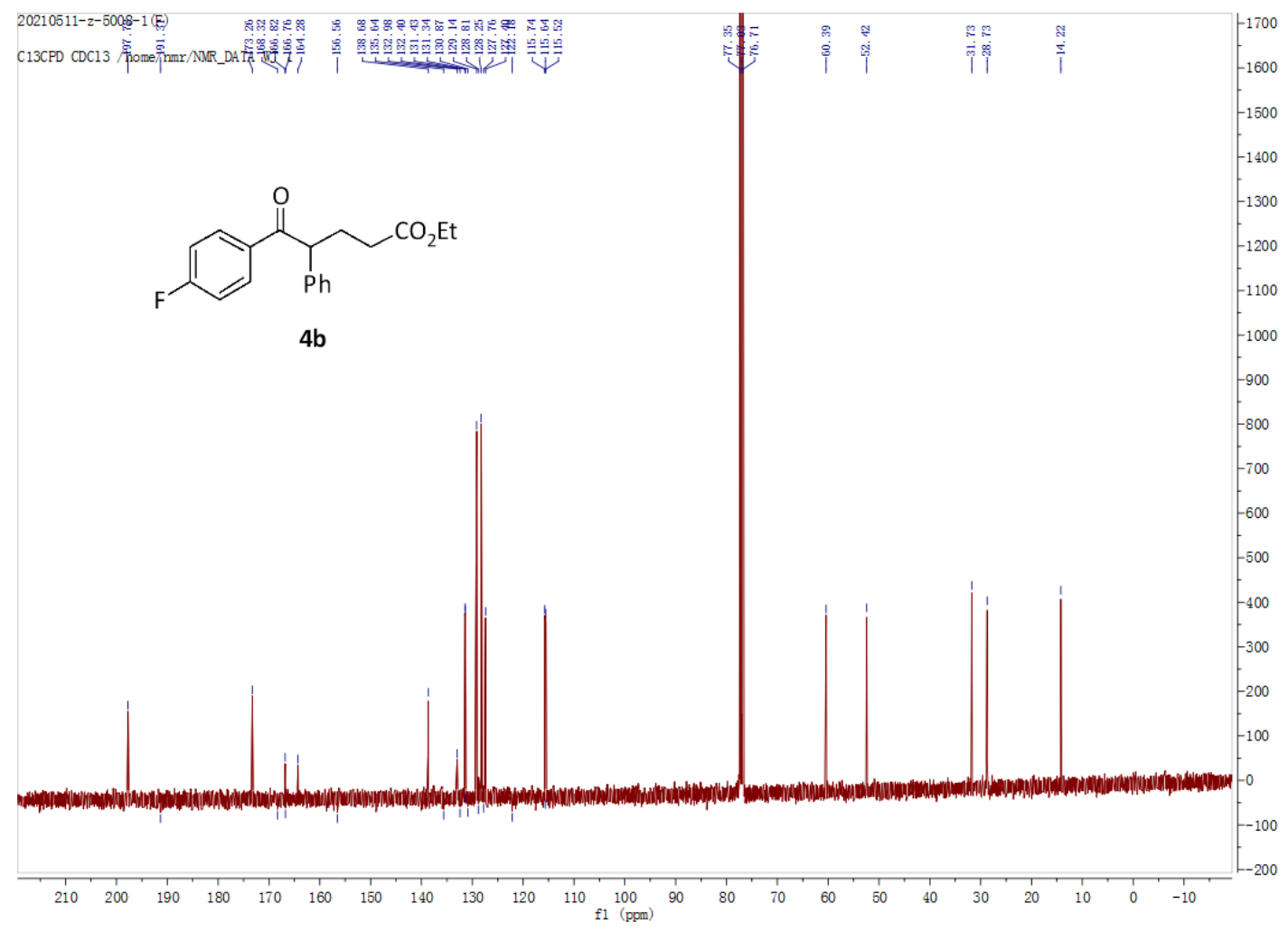


Compound 4c

${ }^{1} \mathrm{H} \mathrm{NMR}\left(\mathrm{CDCl}_{3}, 400 \mathrm{MHz}\right)$

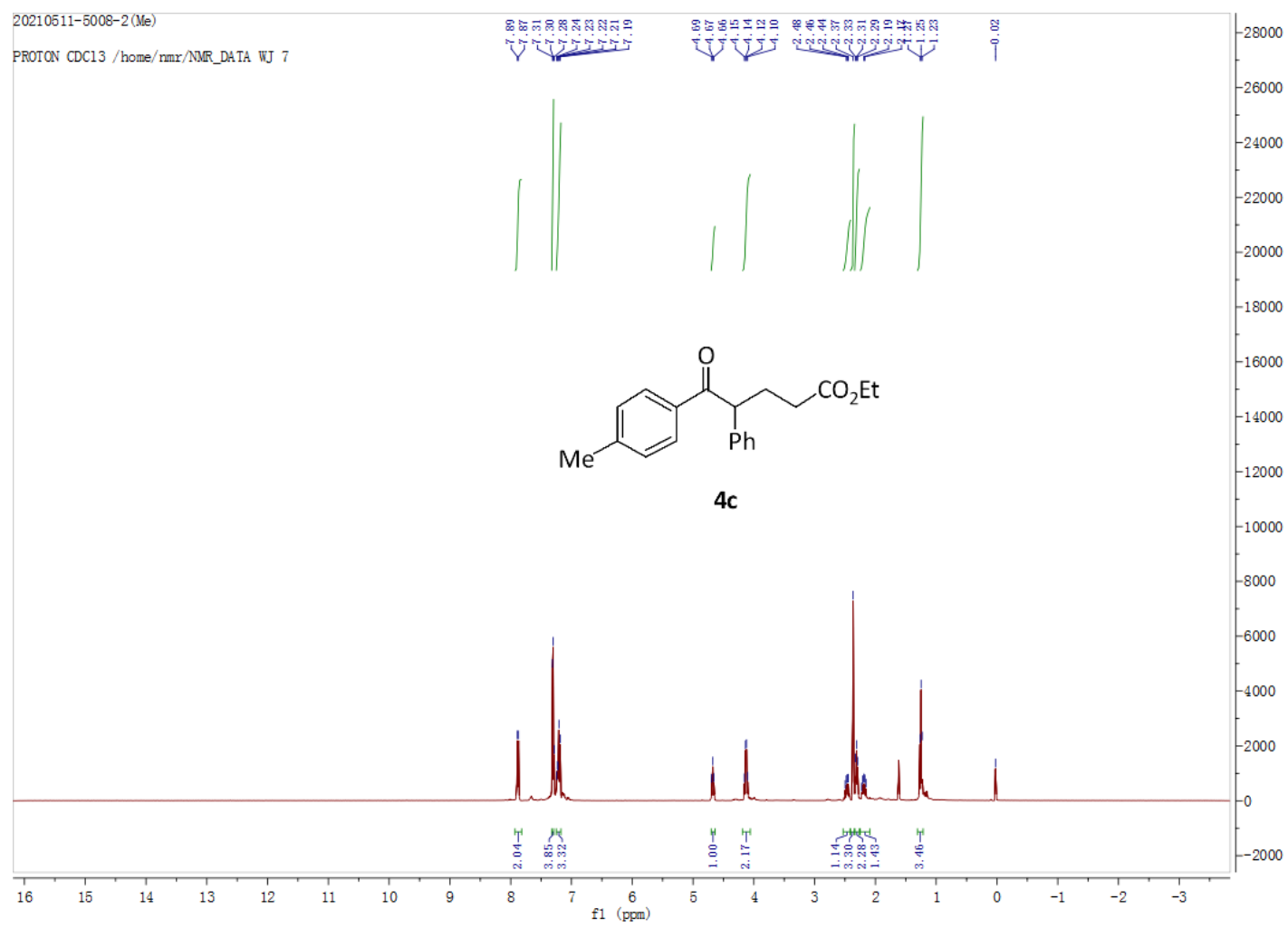

${ }^{13} \mathrm{C} \mathrm{NMR}\left(\mathrm{CDCl}_{3}, 100 \mathrm{MHz}\right)$

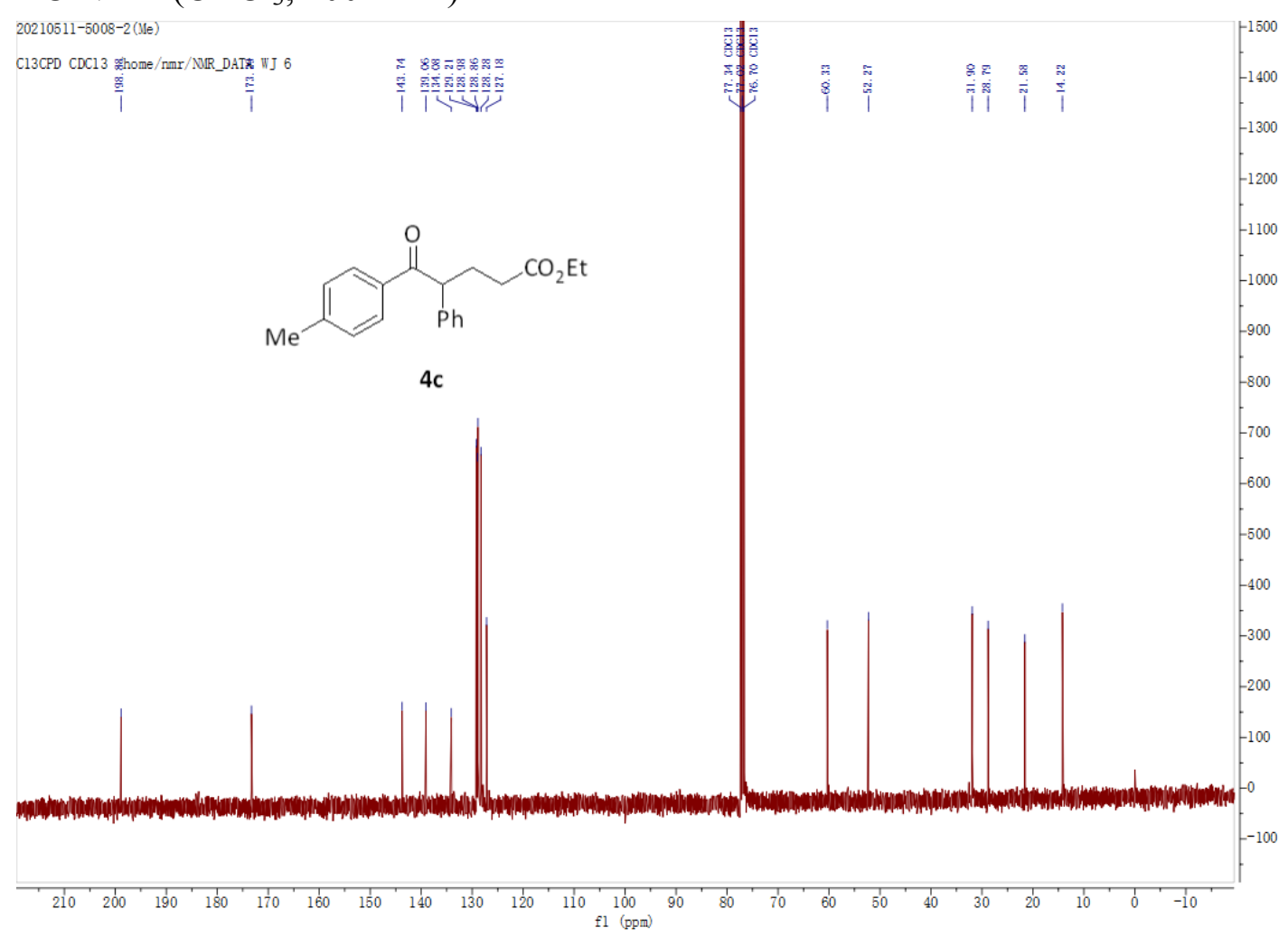




\section{Compound 4d}

${ }^{1} \mathrm{H} \mathrm{NMR}\left(\mathrm{CDCl}_{3}, 400 \mathrm{MHz}\right)$

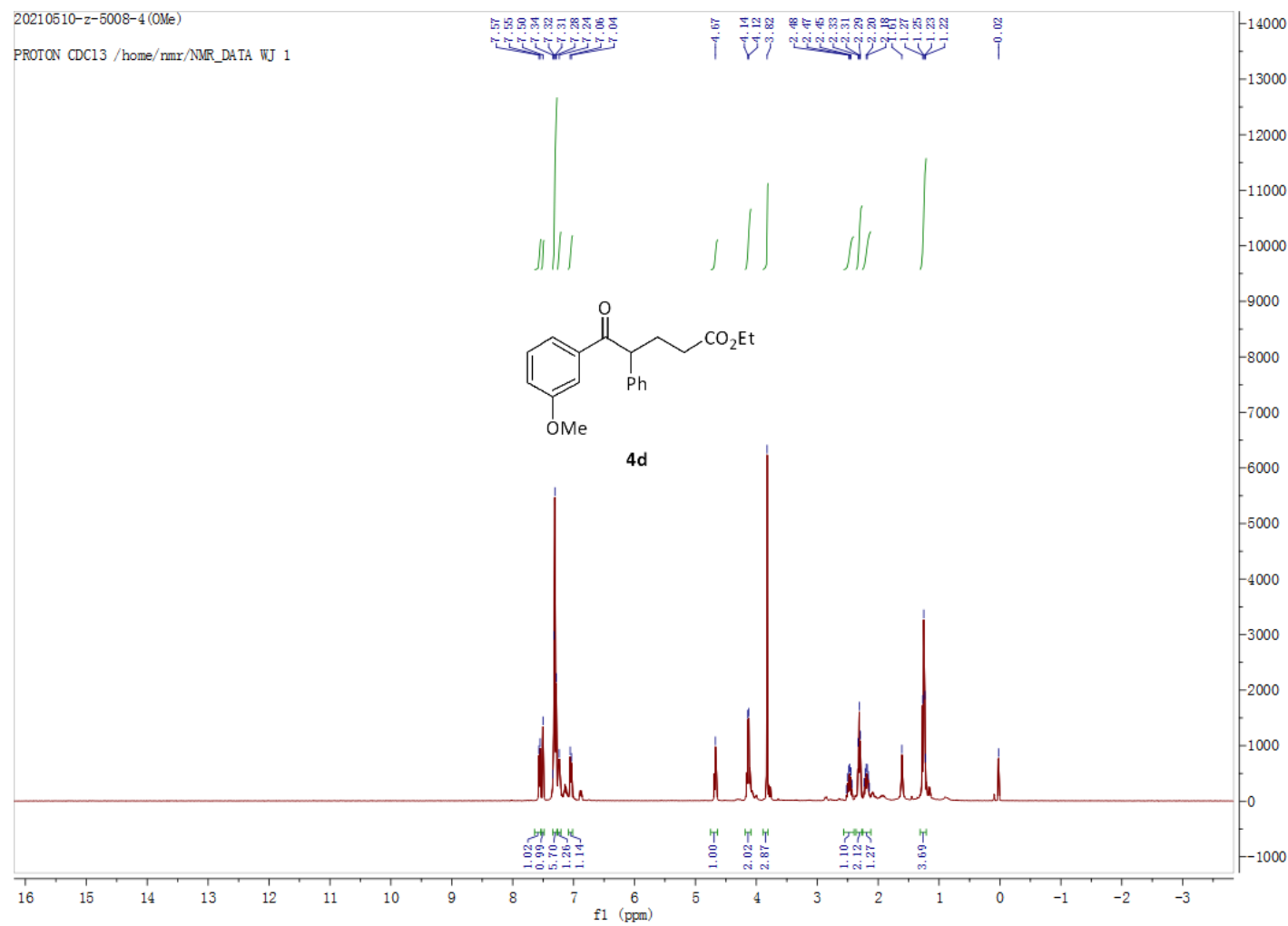

${ }^{13} \mathrm{C}$ NMR $\left(\mathrm{CDCl}_{3}, 100 \mathrm{MHz}\right)$

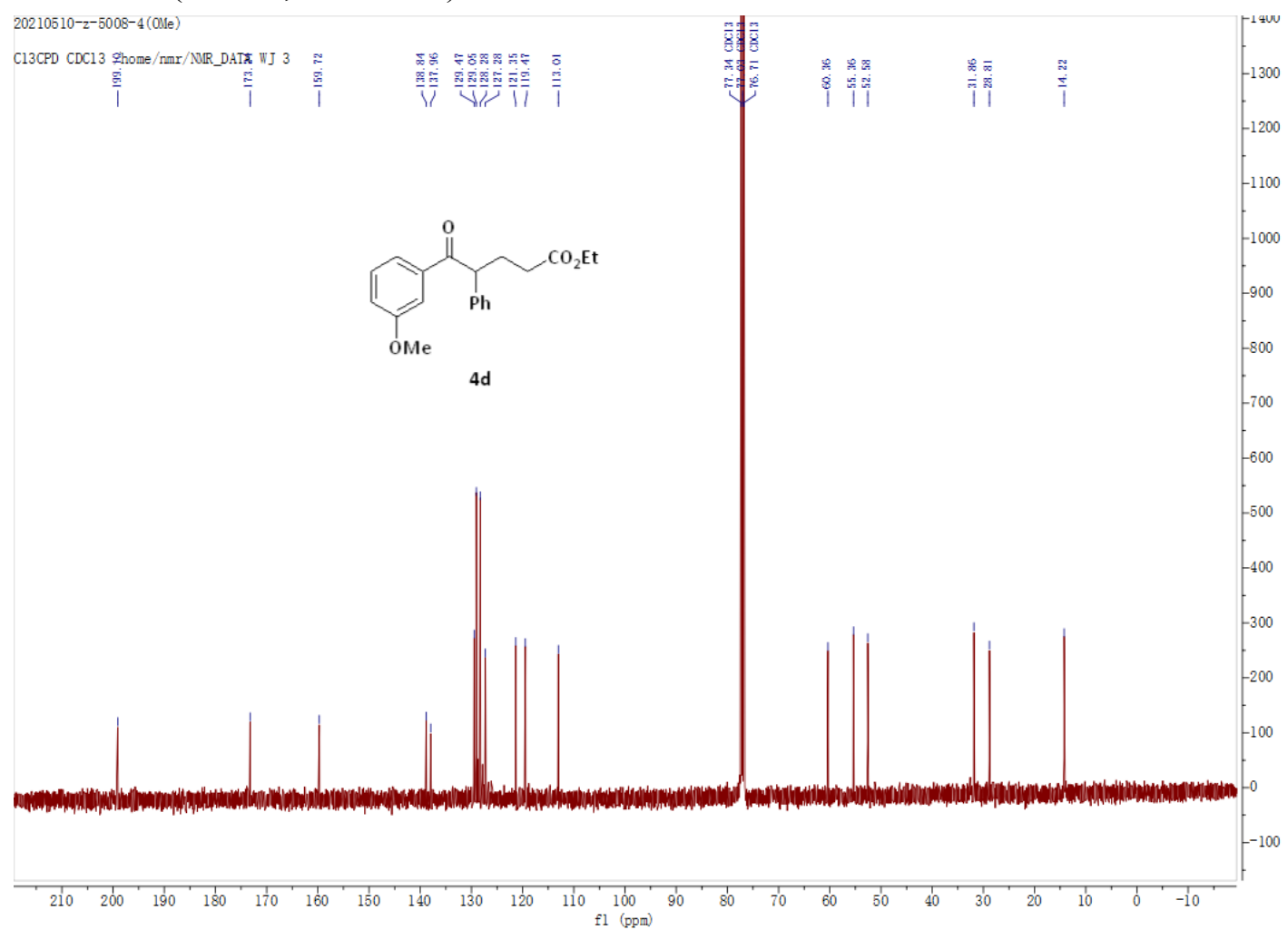




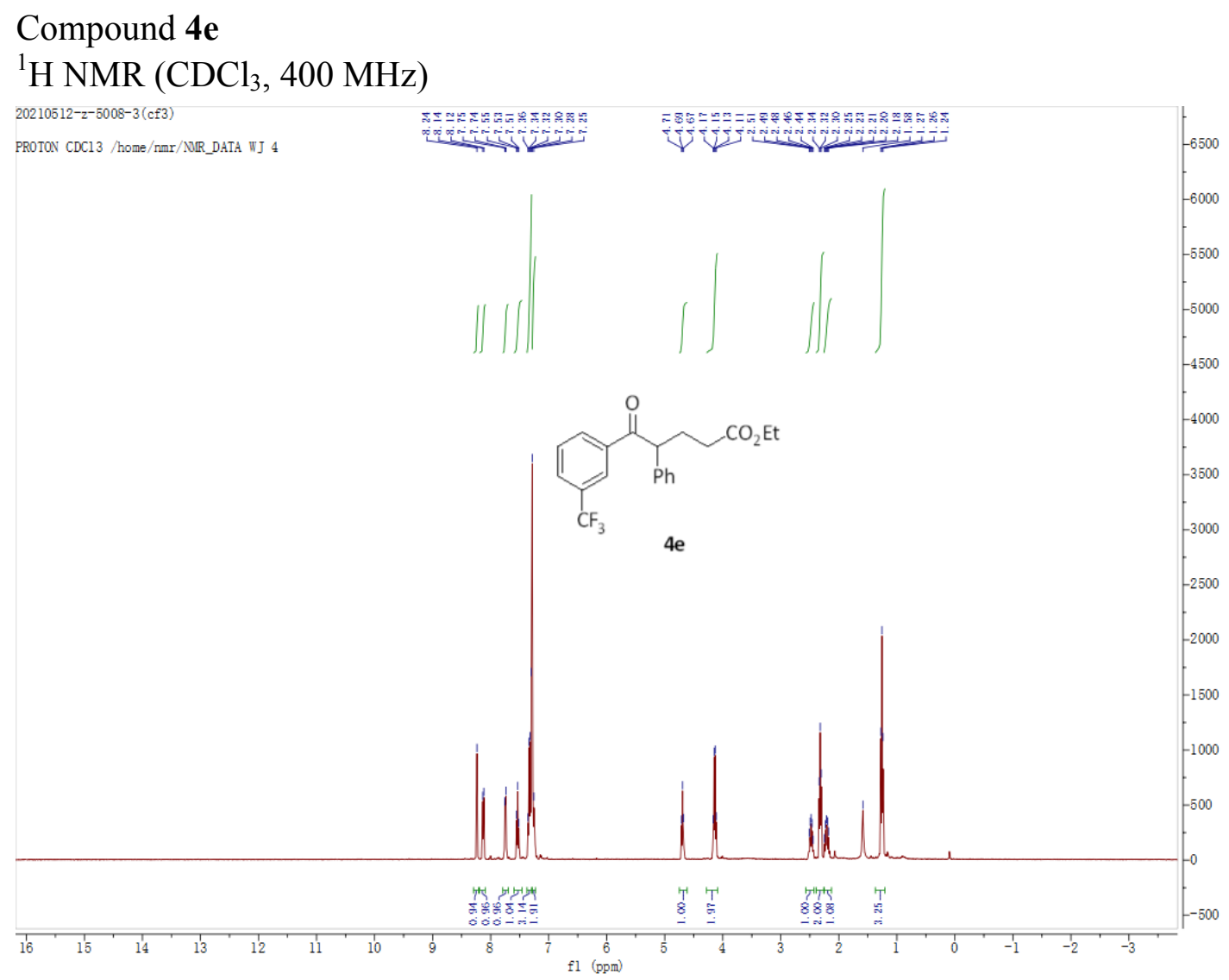

${ }^{19} \mathrm{~F} \mathrm{NMR}\left(\mathrm{CDCl}_{3}, 376 \mathrm{MHz}\right)$

20210512-z-5008-3(cf3)

F19CPD CDC13 /home/nmr/NMR_DATA WJ 4

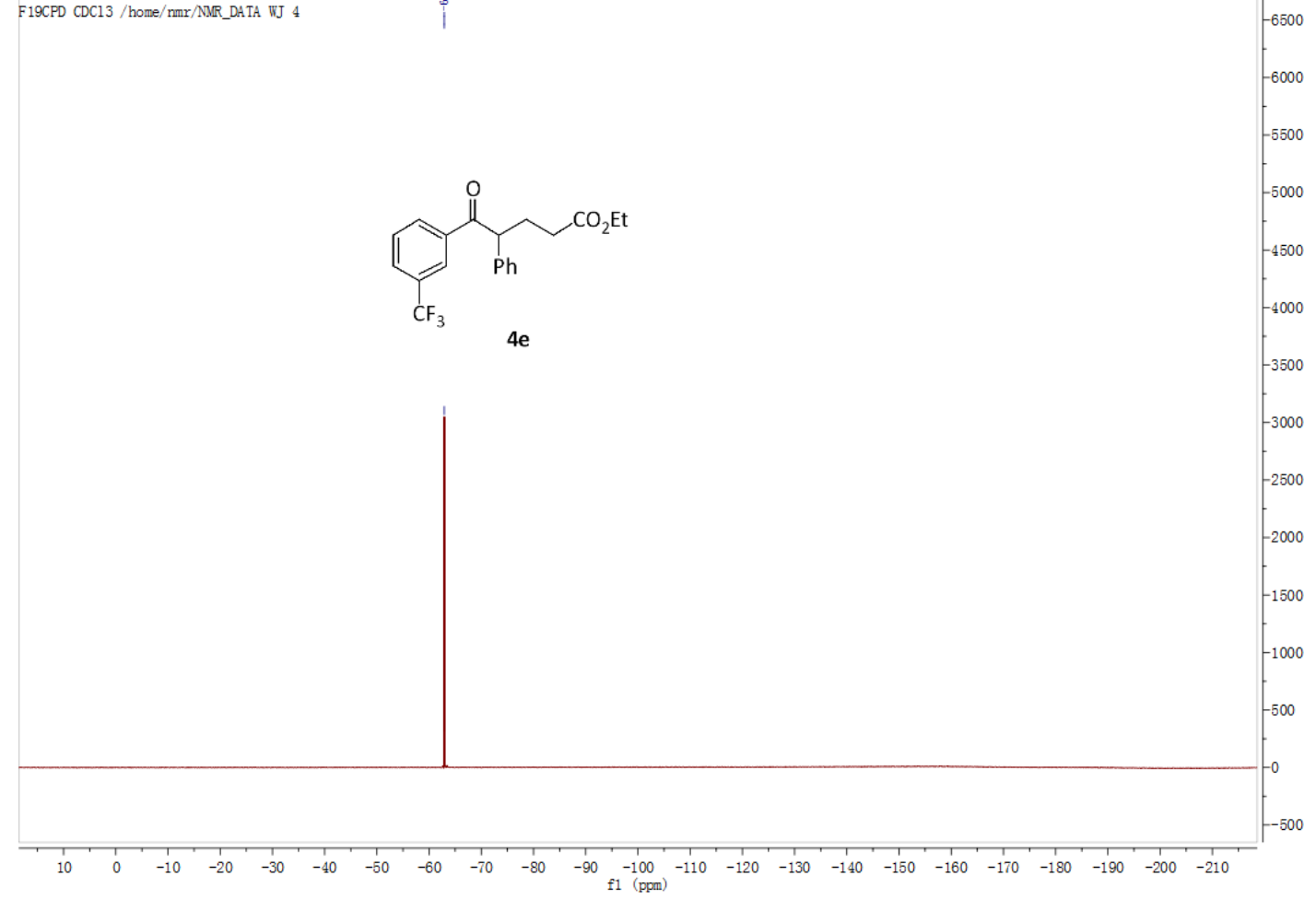

${ }^{13} \mathrm{C} \mathrm{NMR}\left(\mathrm{CDCl}_{3}, 100 \mathrm{MHz}\right)$ 


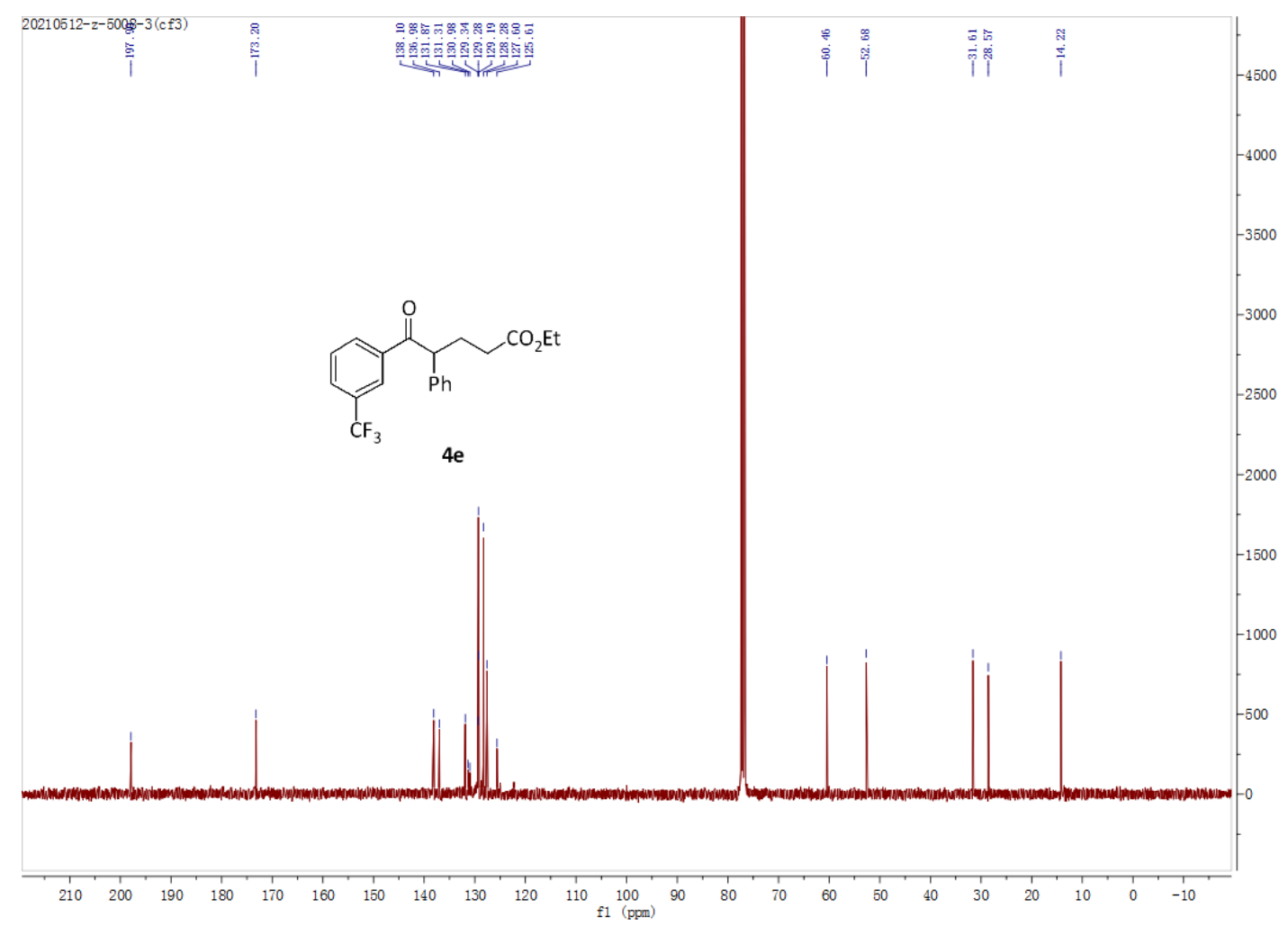




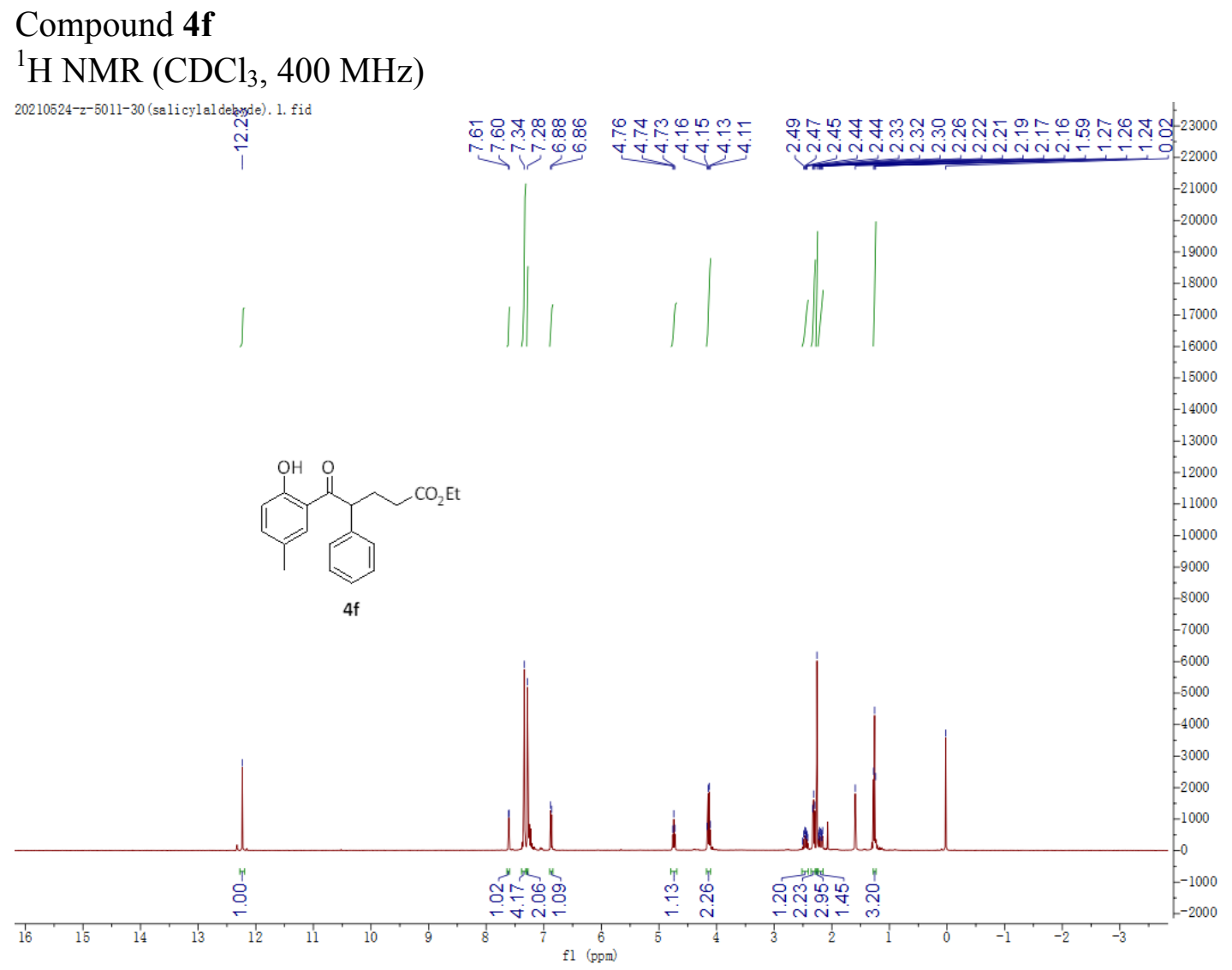

${ }^{13} \mathrm{C}$ NMR $\left(\mathrm{CDCl}_{3}, 100 \mathrm{MHz}\right)$

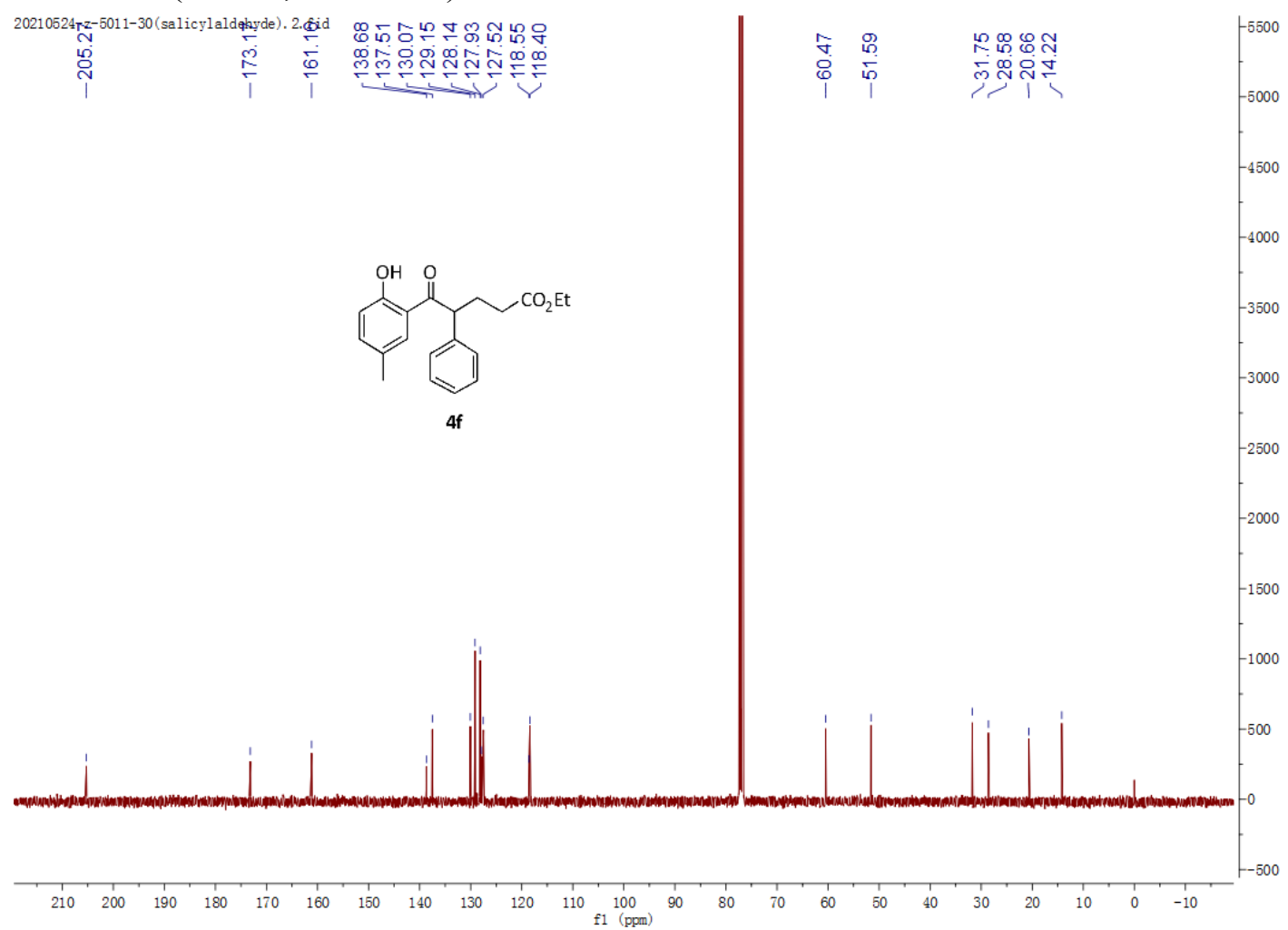




\section{Compound 4g}

${ }^{1} \mathrm{H} \mathrm{NMR}\left(\mathrm{CDCl}_{3}, 400 \mathrm{MHz}\right)$

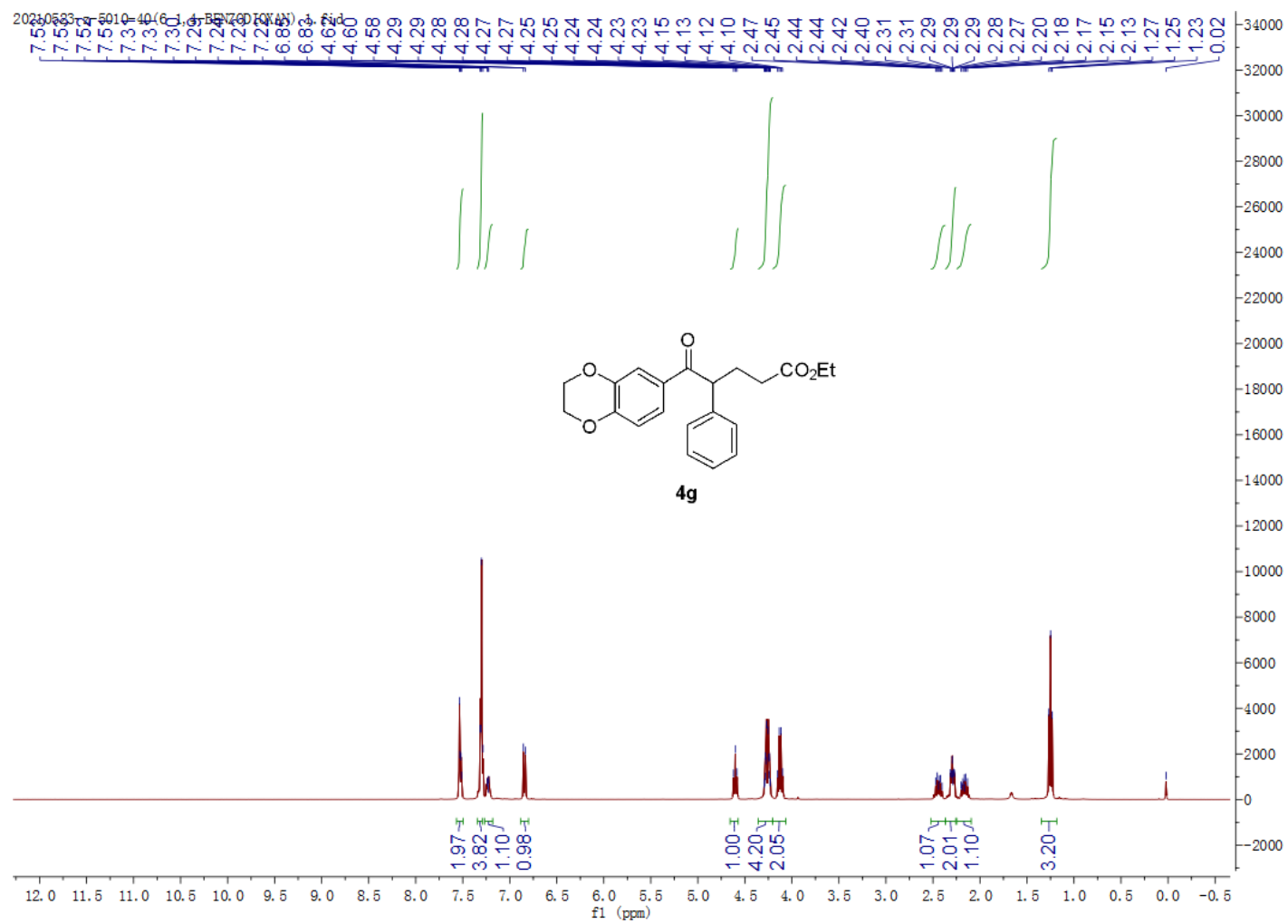

${ }^{13} \mathrm{C} \mathrm{NMR}\left(\mathrm{CDCl}_{3}, 100 \mathrm{MHz}\right)$

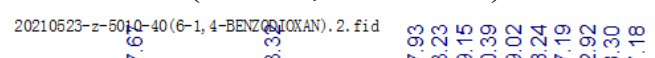
।

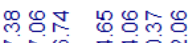

№ Gं

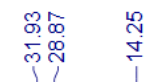

6500

6000

5500

5000<smiles>CCOC(=O)CCC(C(=O)c1ccc2c(c1)OCCO2)c1ccccc1</smiles>

$4 \mathrm{~g}$

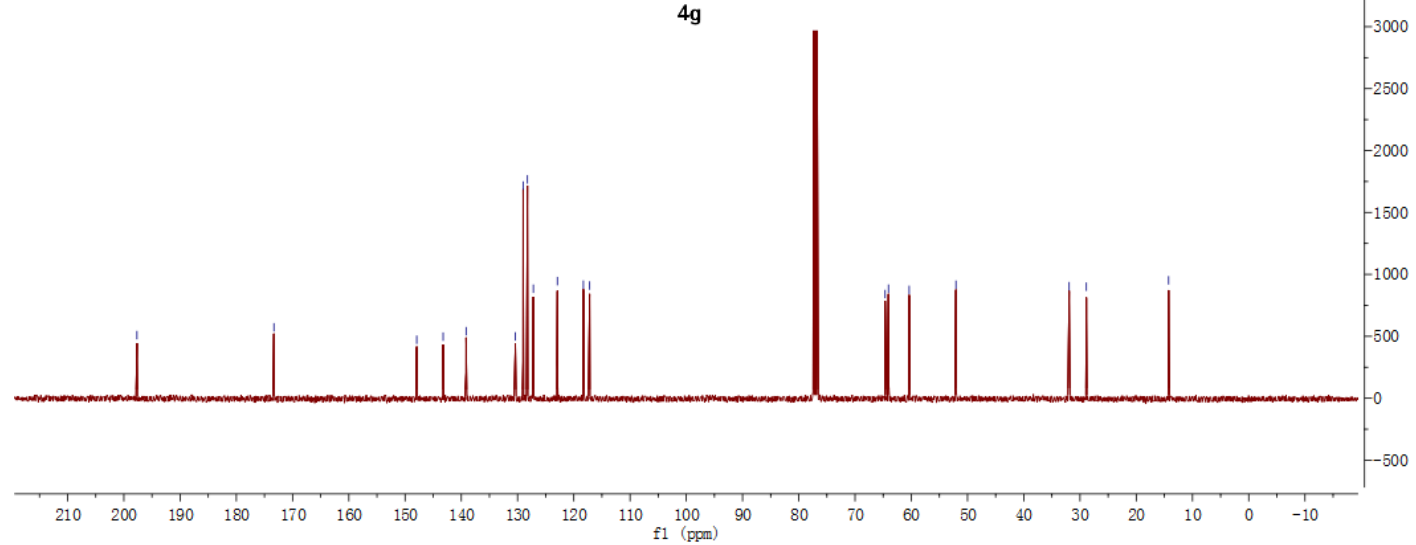




\section{Compound $\mathbf{4 h}$}

${ }^{1} \mathrm{H} \mathrm{NMR}\left(\mathrm{CDCl}_{3}, 400 \mathrm{MHz}\right)$

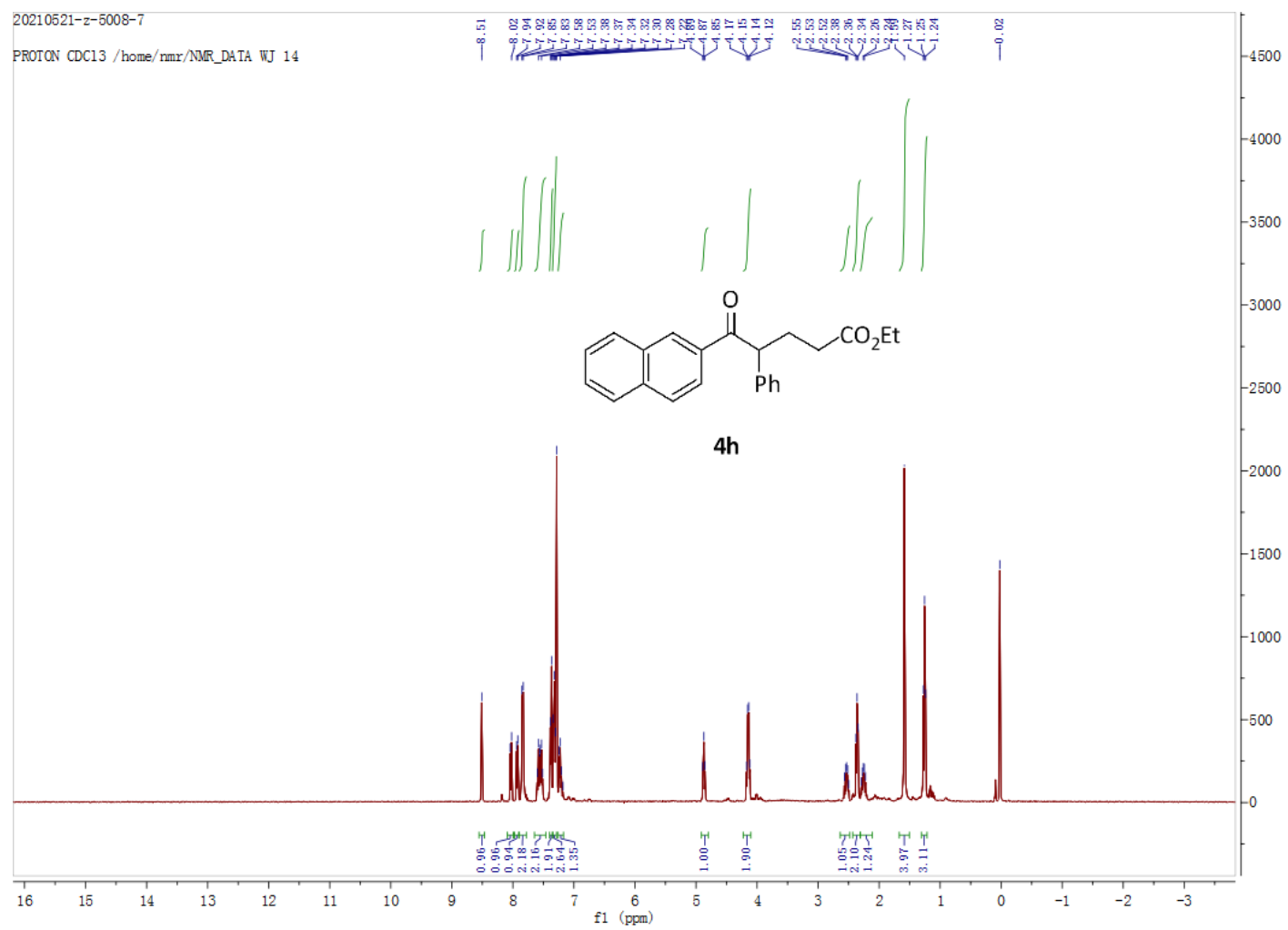

${ }^{13} \mathrm{C} \mathrm{NMR}\left(\mathrm{CDCl}_{3}, 100 \mathrm{MHz}\right)$

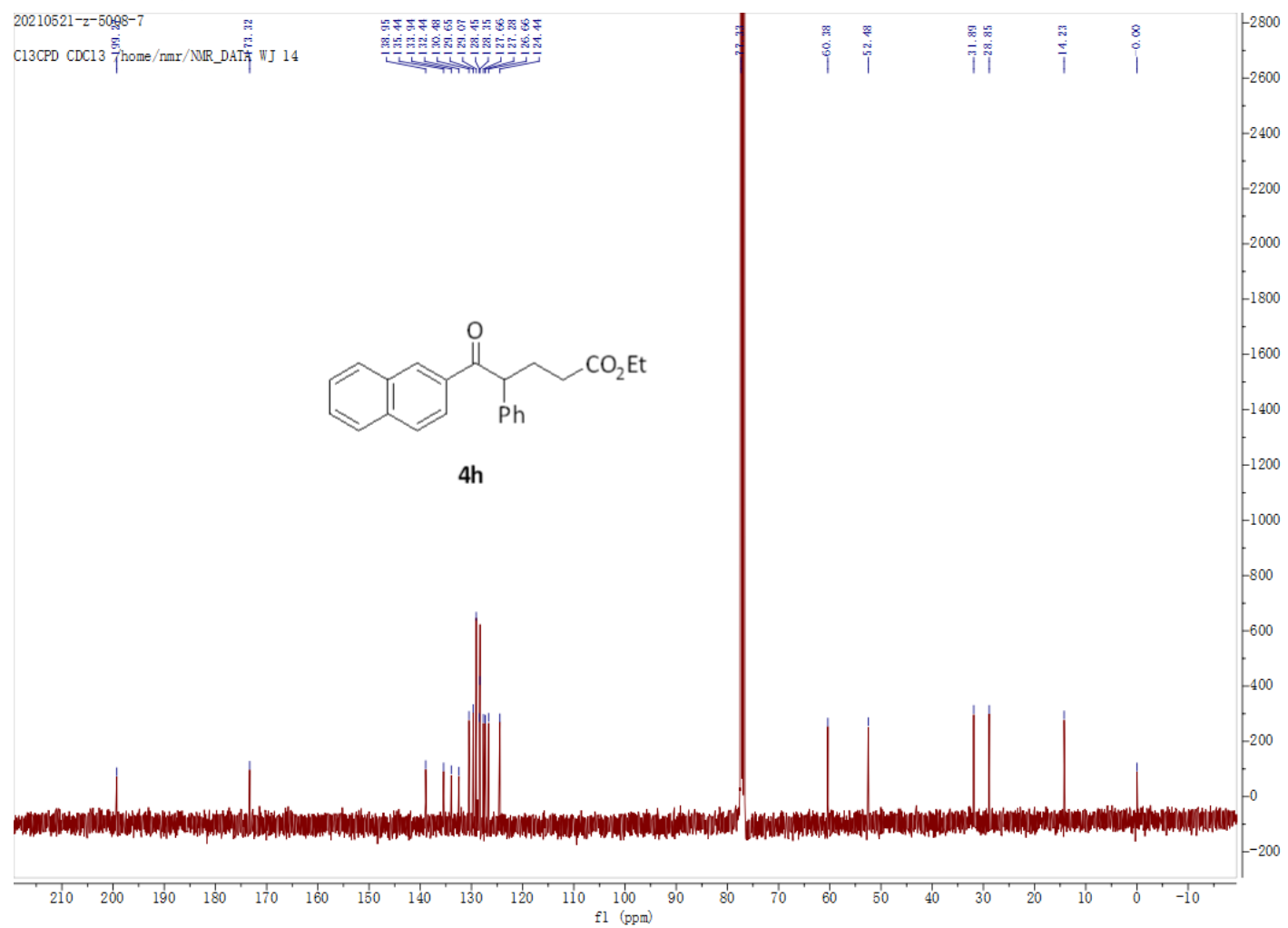




\section{Compound 4i}

${ }^{1} \mathrm{H} \mathrm{NMR}\left(\mathrm{CDCl}_{3}, 400 \mathrm{MHz}\right)$

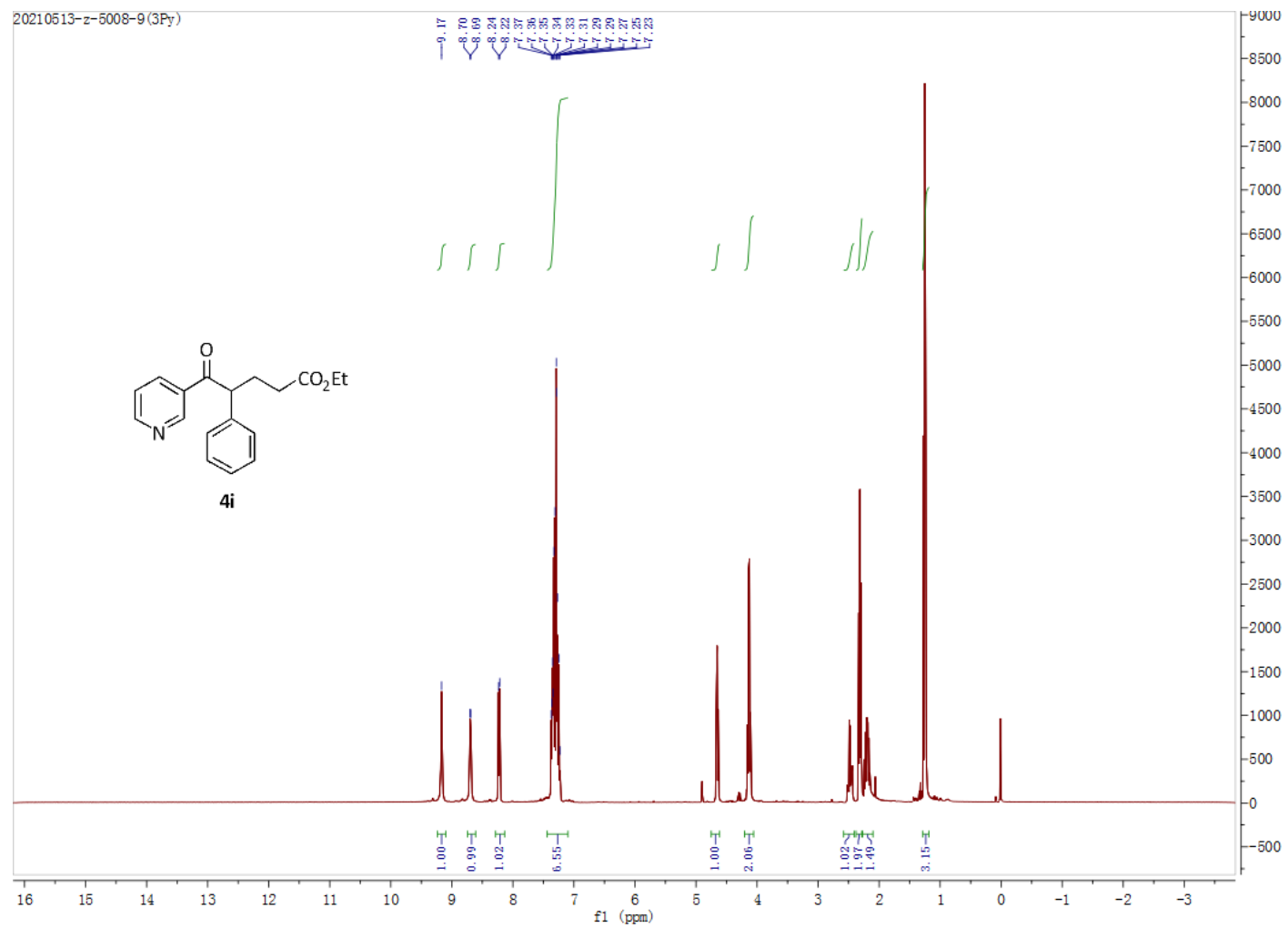

${ }^{13} \mathrm{C}$ NMR $\left(\mathrm{CDCl}_{3}, 100 \mathrm{MHz}\right)$

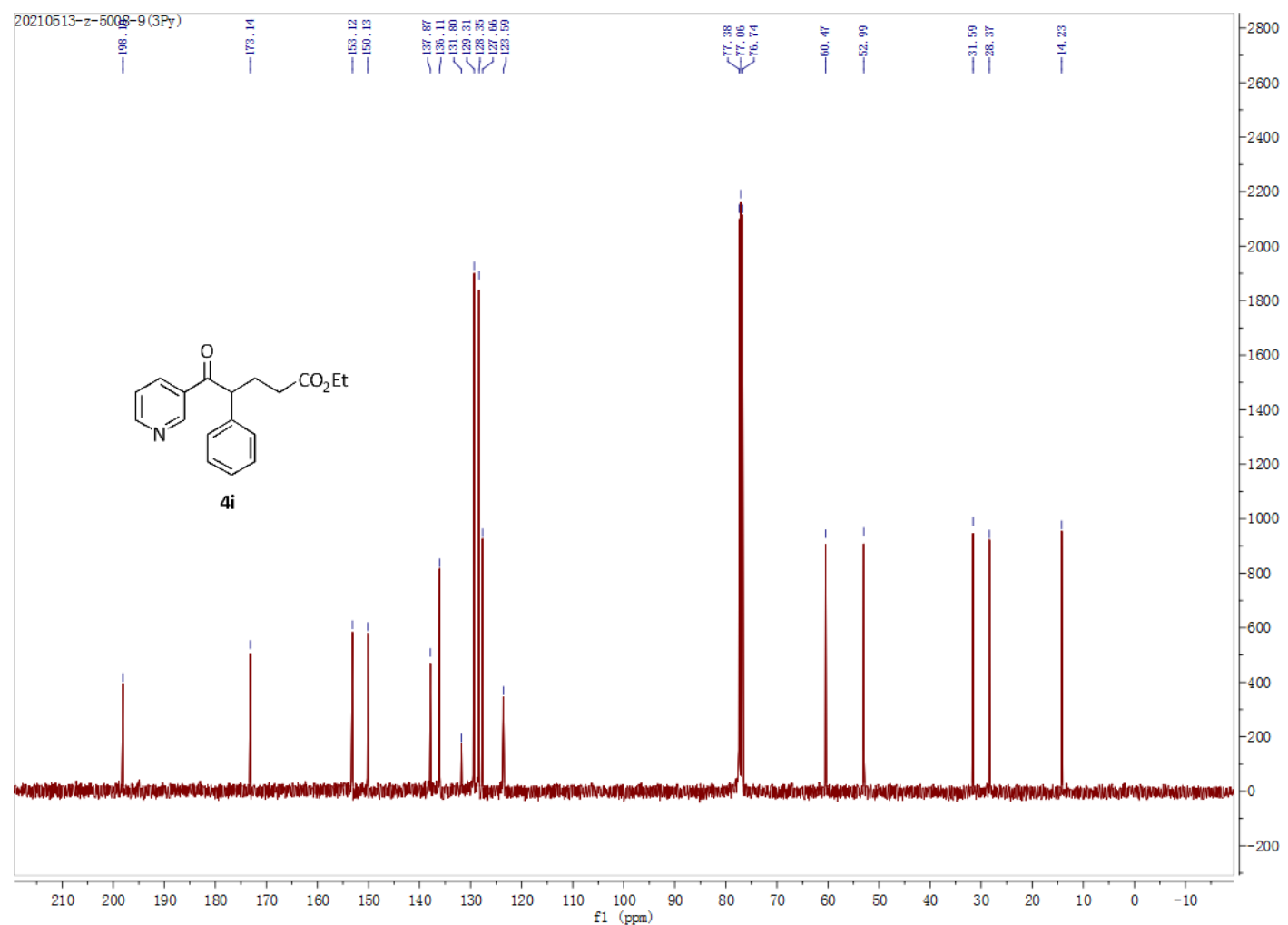




\section{Compound $\mathbf{4 j}$}

${ }^{1} \mathrm{H} \mathrm{NMR}\left(\mathrm{CDCl}_{3}, 400 \mathrm{MHz}\right)$

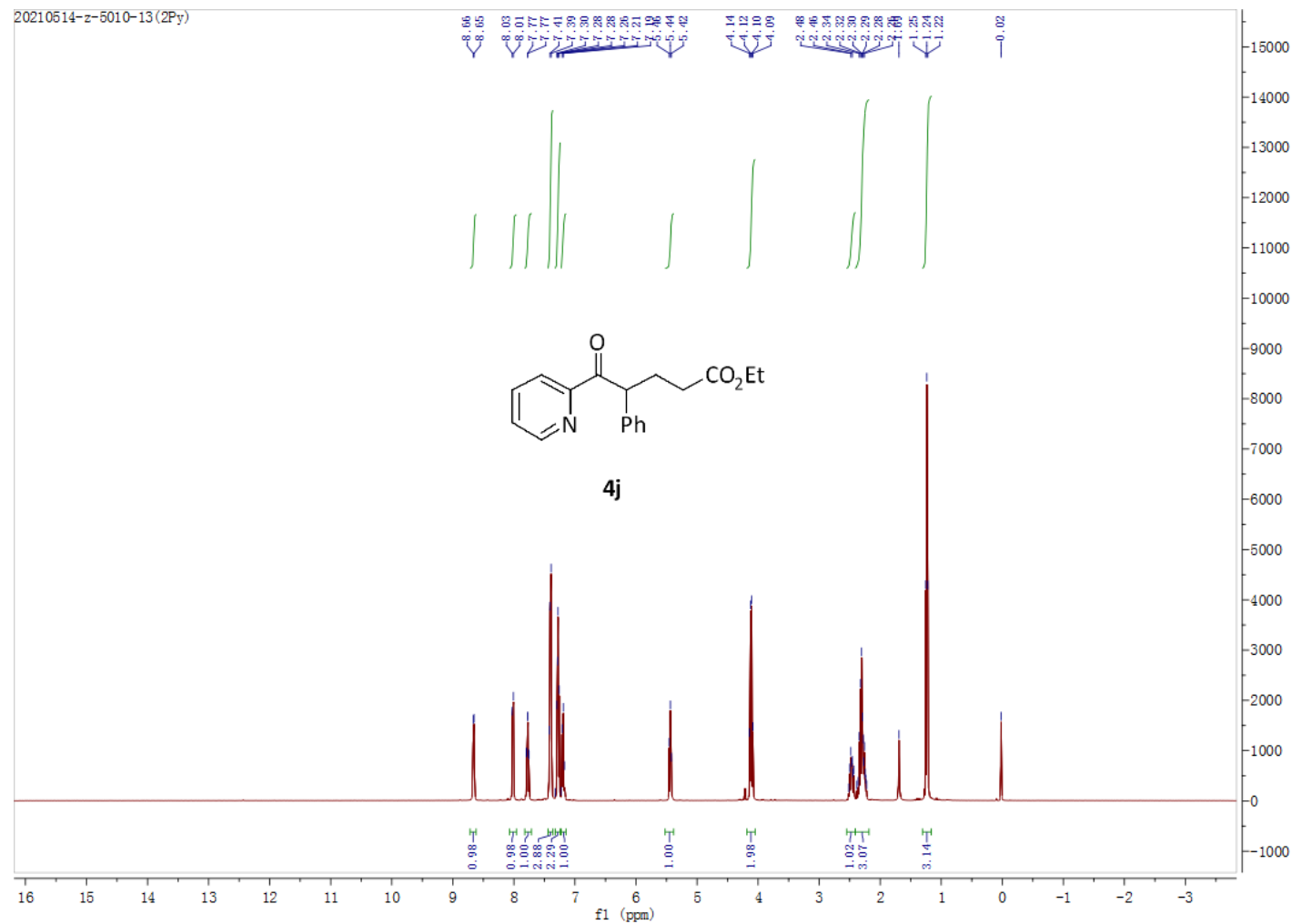

${ }^{13} \mathrm{C}$ NMR $\left(\mathrm{CDCl}_{3}, 100 \mathrm{MHz}\right)$

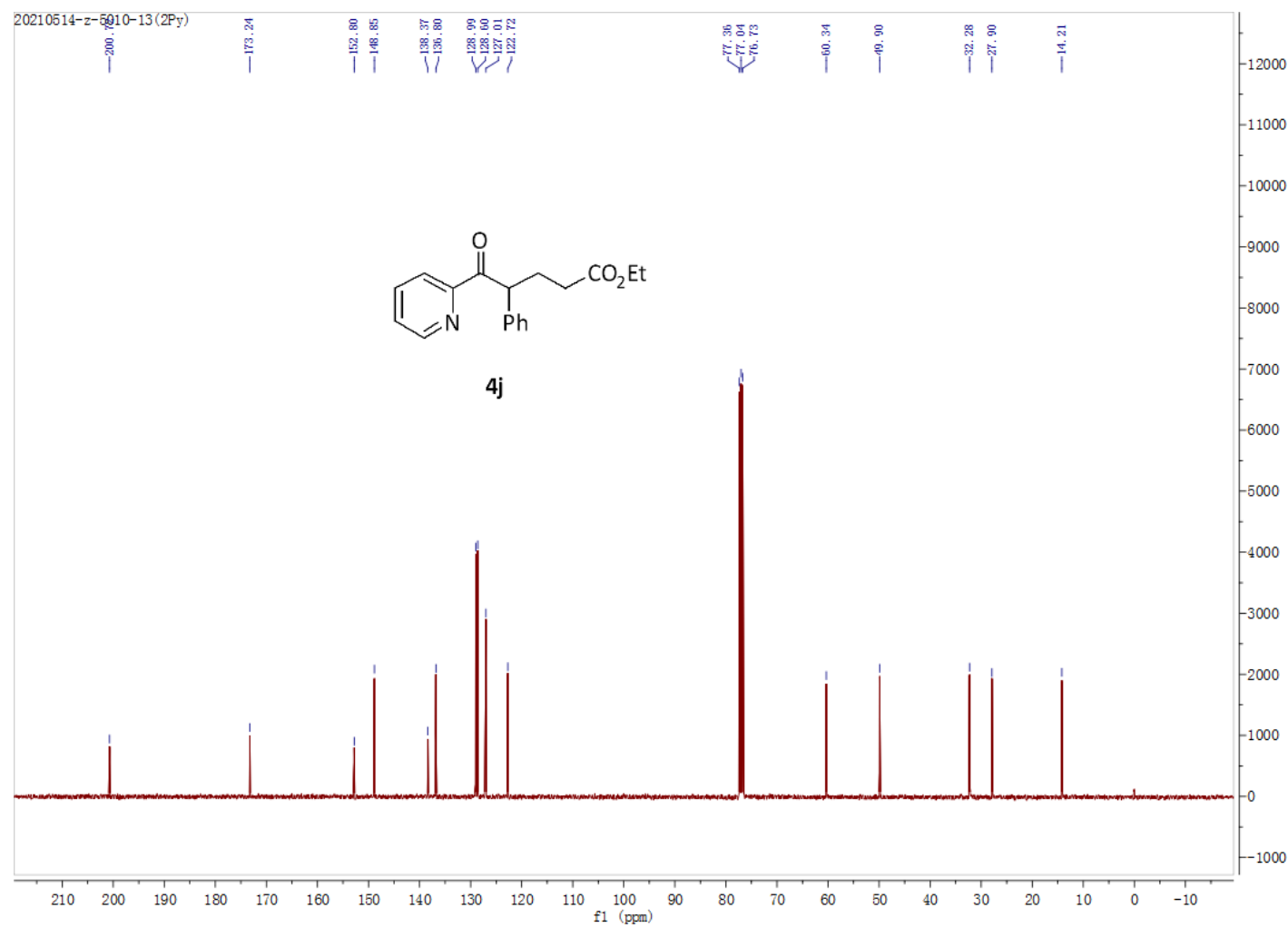




\section{Compound 4k}

${ }^{1} \mathrm{H} \mathrm{NMR}\left(\mathrm{CDCl}_{3}, 400 \mathrm{MHz}\right)$

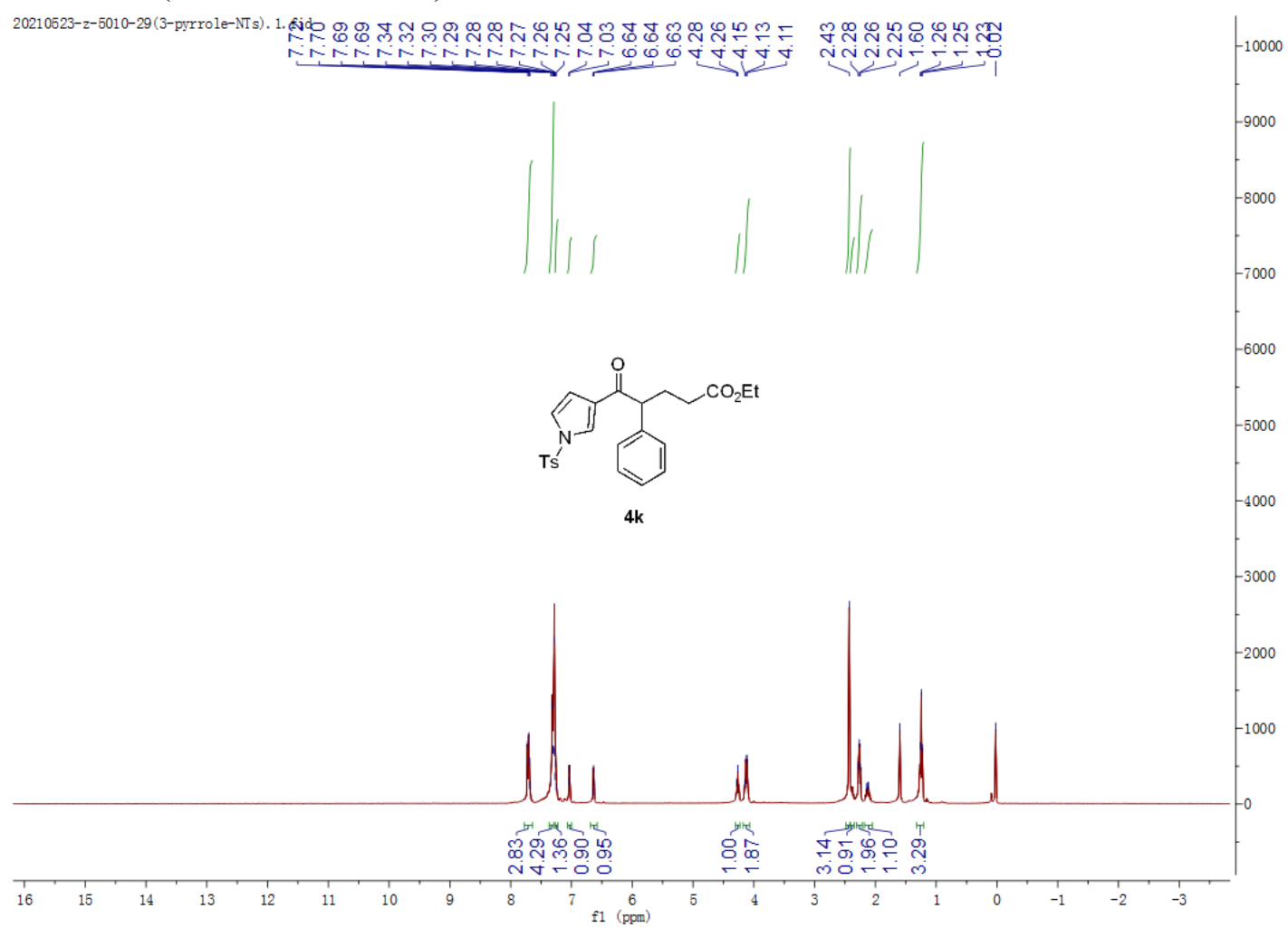

${ }^{13} \mathrm{C} \mathrm{NMR}\left(\mathrm{CDCl}_{3}, 100 \mathrm{MHz}\right)$ 每

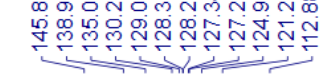

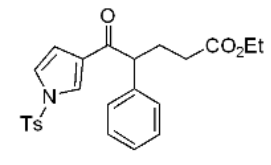

$4 \mathrm{k}$

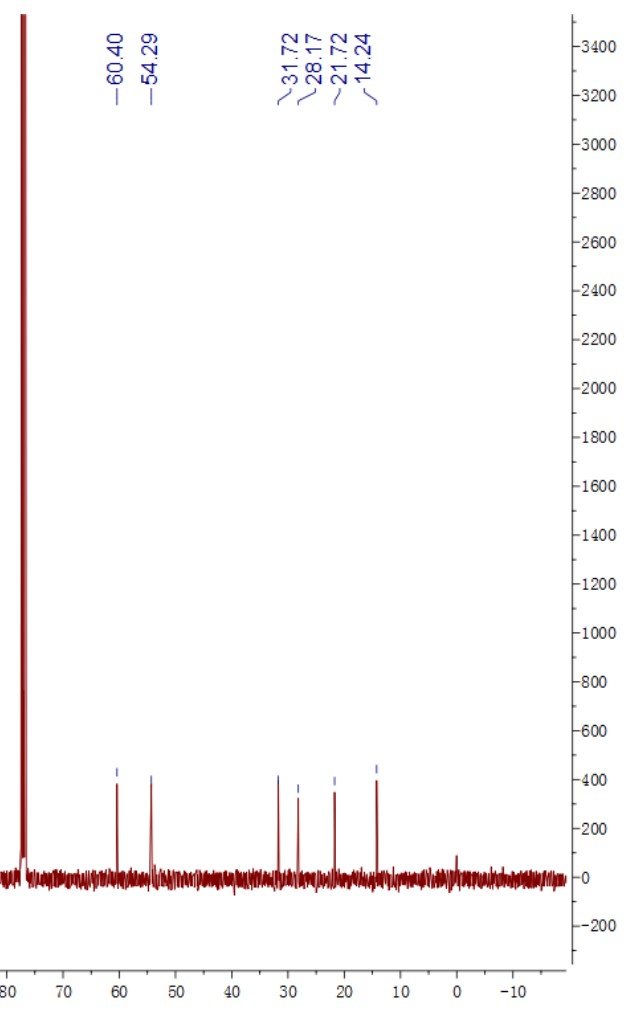




\section{Compound 4l}

${ }^{1} \mathrm{H} \mathrm{NMR}\left(\mathrm{CDCl}_{3}, 400 \mathrm{MHz}\right)$

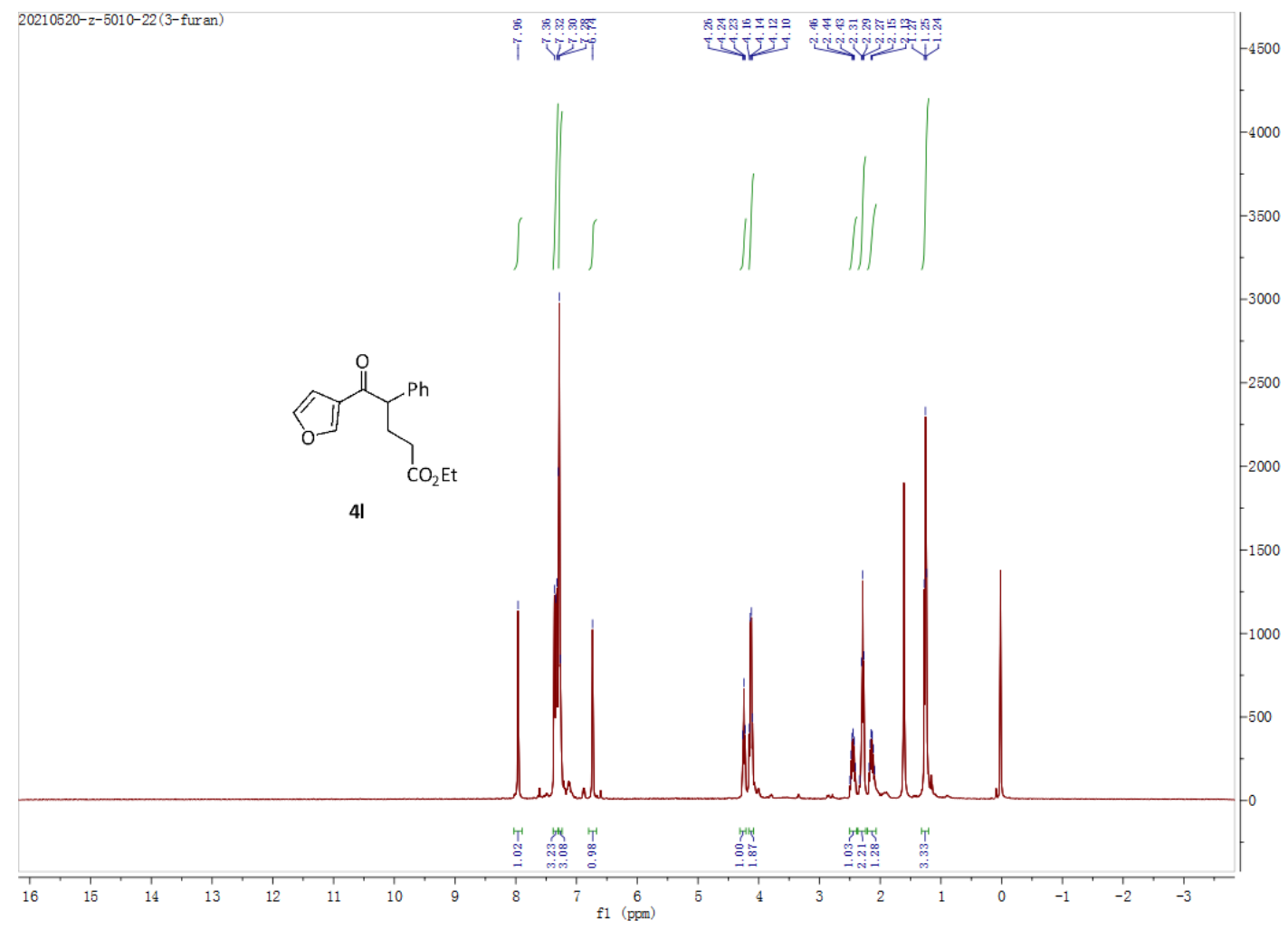

${ }^{13} \mathrm{C} \mathrm{NMR}\left(\mathrm{CDCl}_{3}, 100 \mathrm{MHz}\right)$

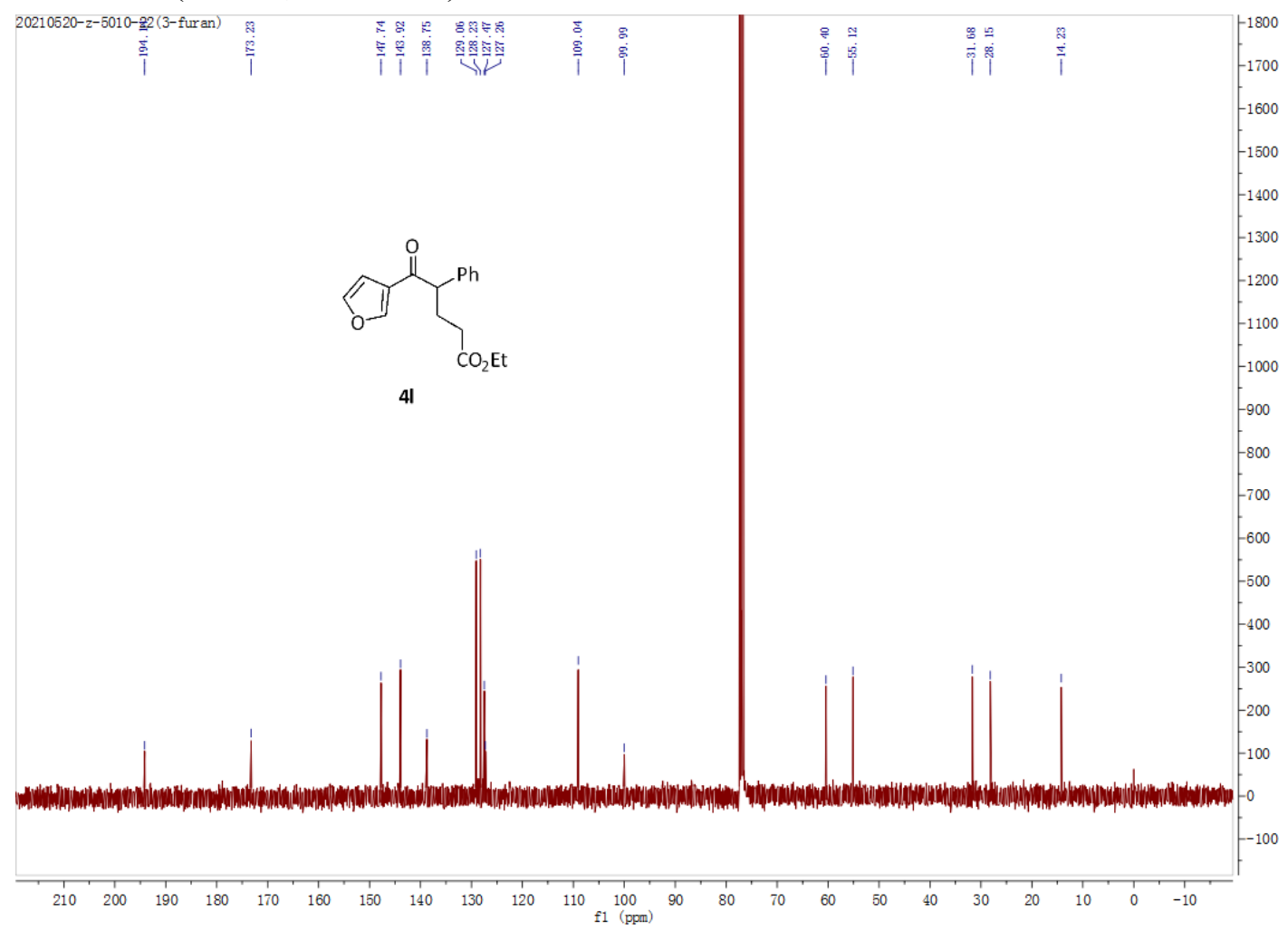




\section{Compound 4m}

${ }^{1} \mathrm{H} \mathrm{NMR}\left(\mathrm{CDCl}_{3}, 400 \mathrm{MHz}\right)$

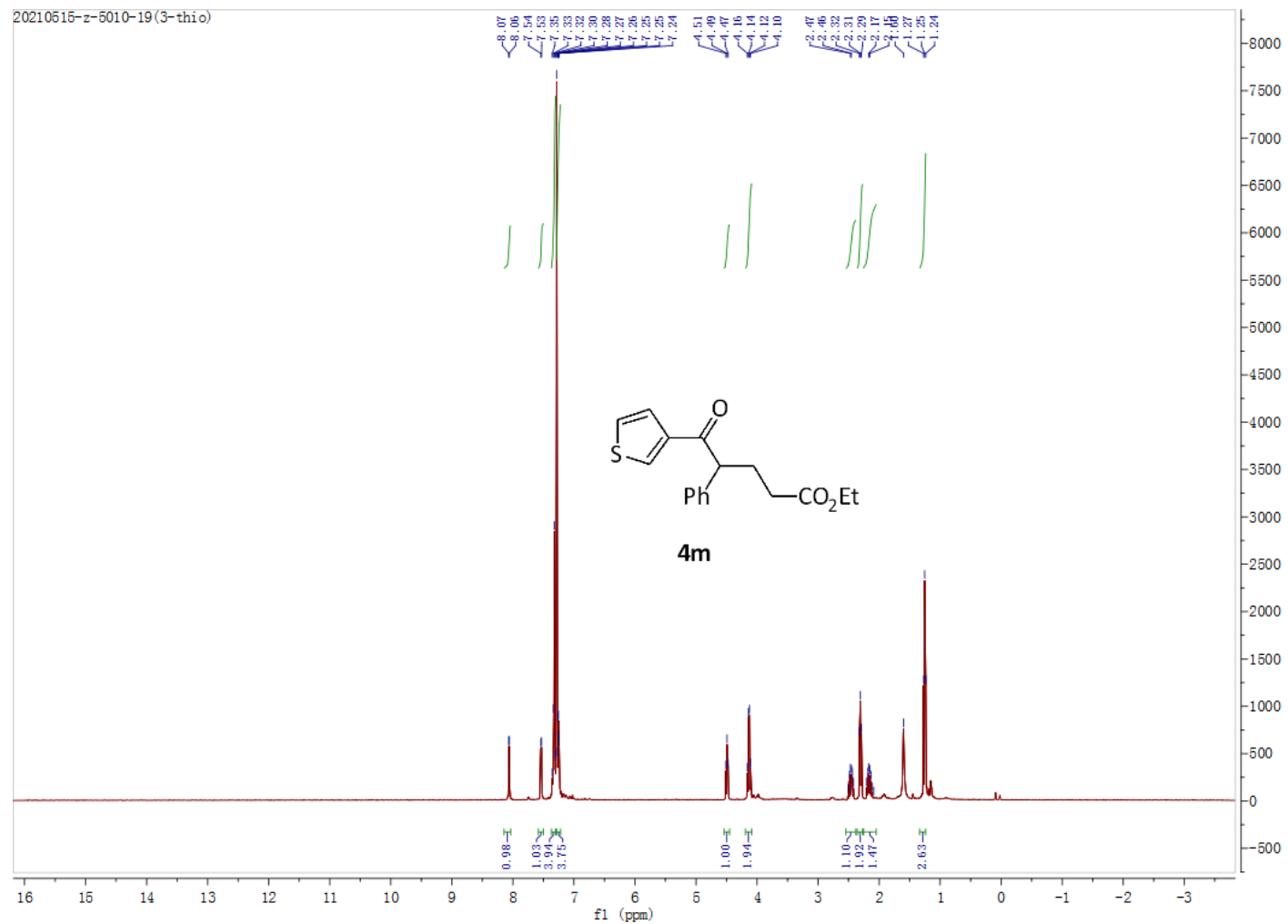

${ }^{13} \mathrm{C} \mathrm{NMR}\left(\mathrm{CDCl}_{3}, 100 \mathrm{MHz}\right)$

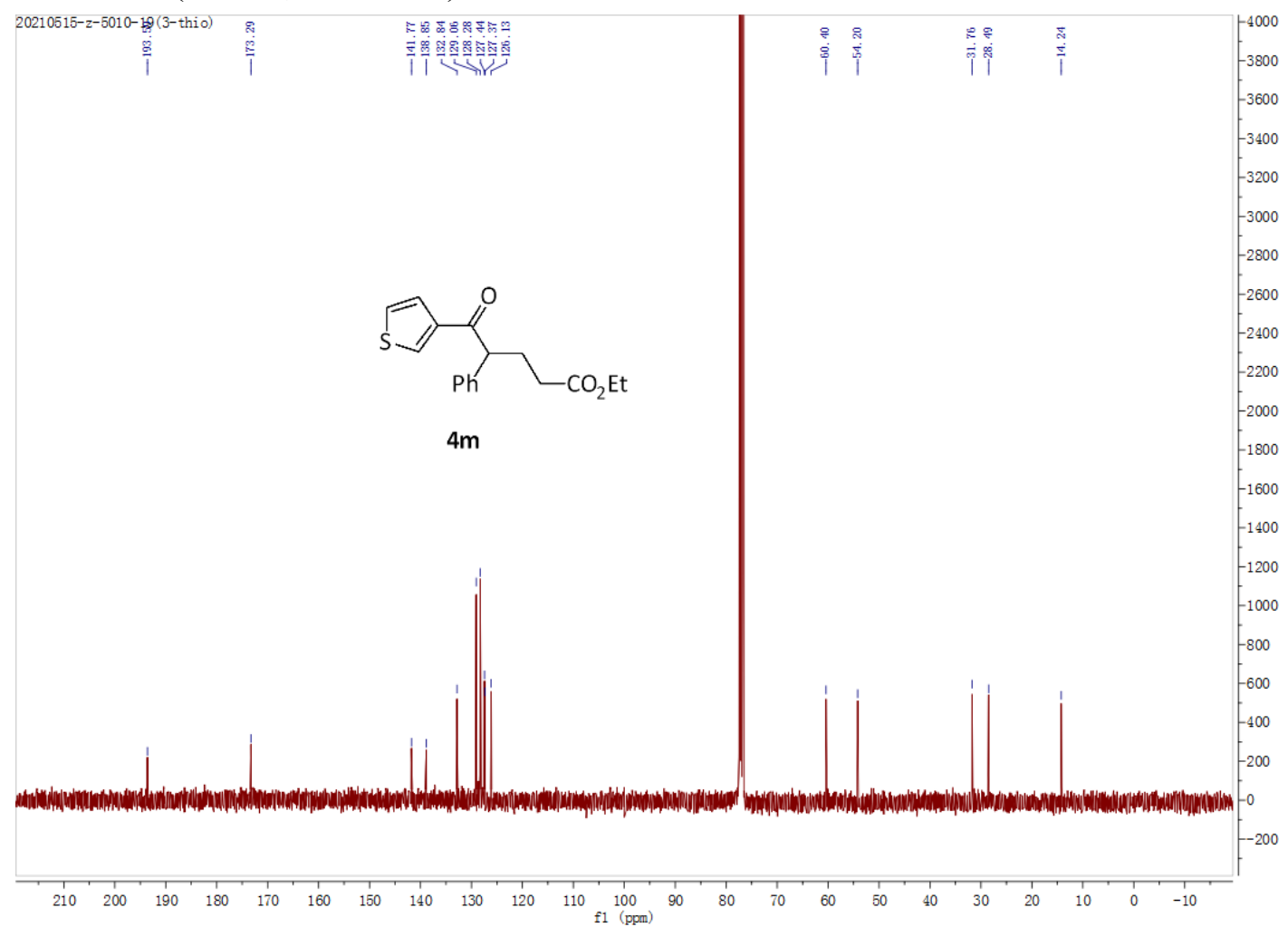




\section{Compound 4n}

${ }^{1} \mathrm{H} \mathrm{NMR}\left(\mathrm{CDCl}_{3}, 400 \mathrm{MHz}\right)$

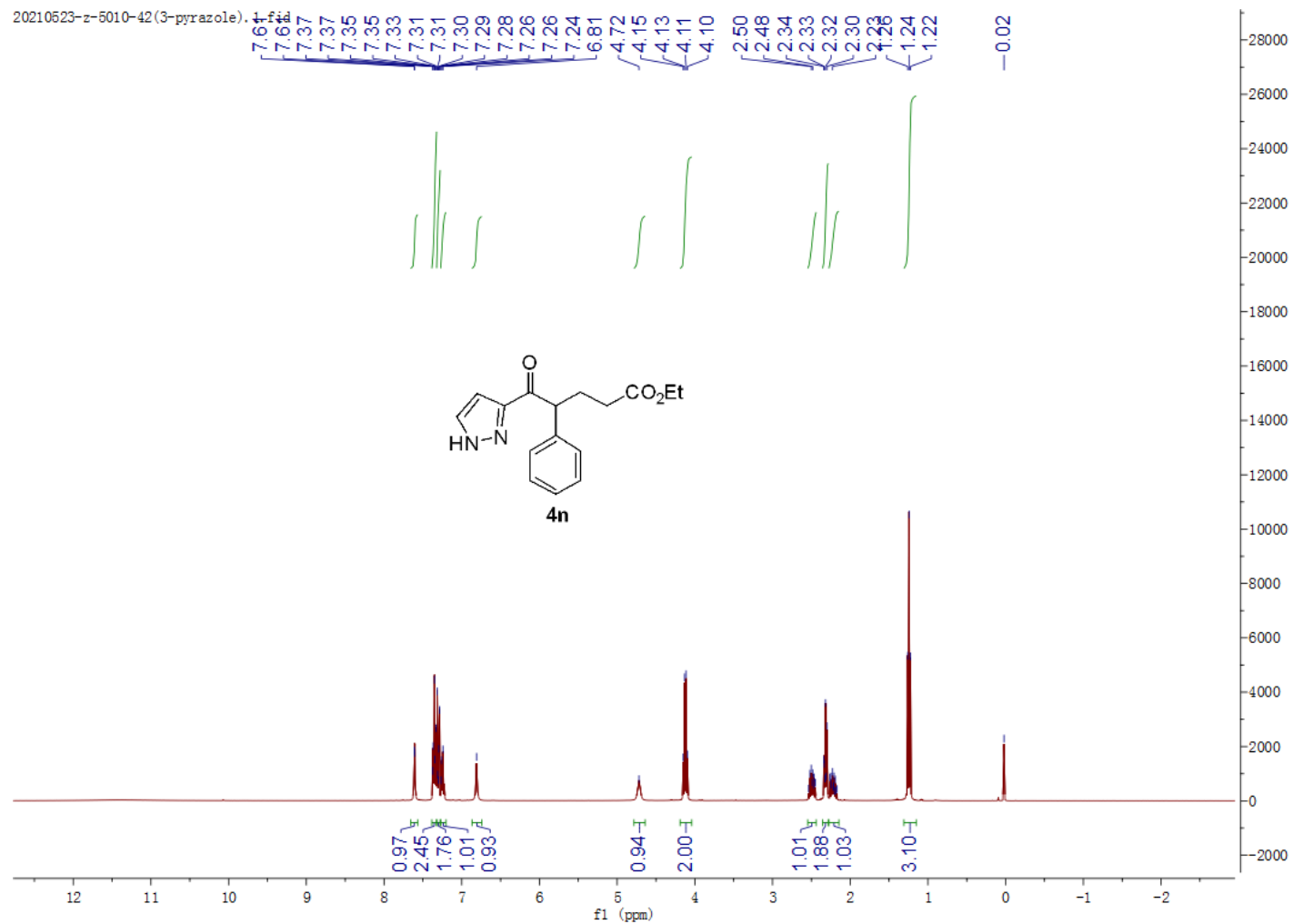

${ }^{13} \mathrm{C} \mathrm{NMR}\left(\mathrm{CDCl}_{3}, 100 \mathrm{MHz}\right)$

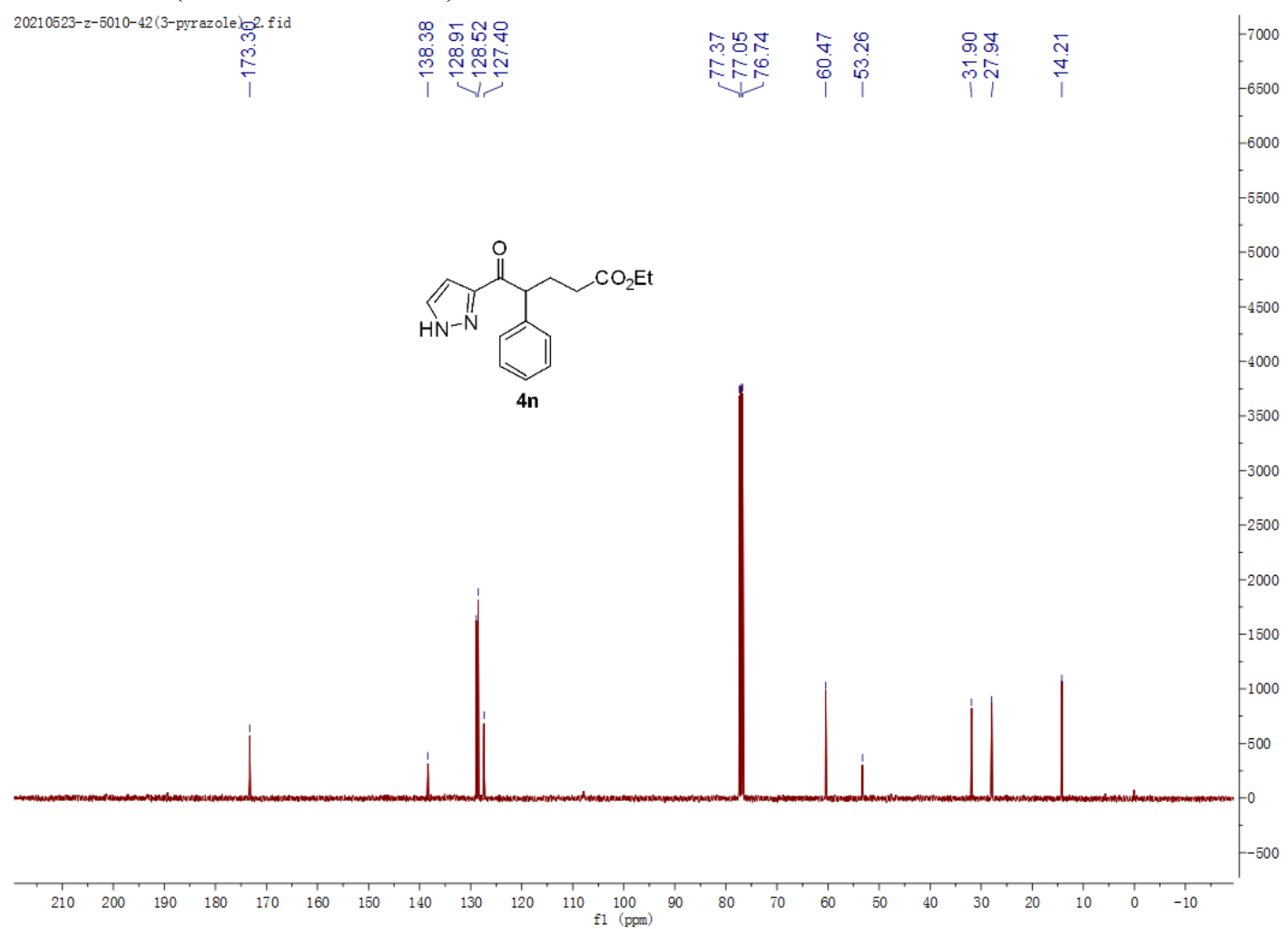




\section{Compound 40}

${ }^{1} \mathrm{H} \mathrm{NMR}\left(\mathrm{CDCl}_{3}, 400 \mathrm{MHz}\right)$

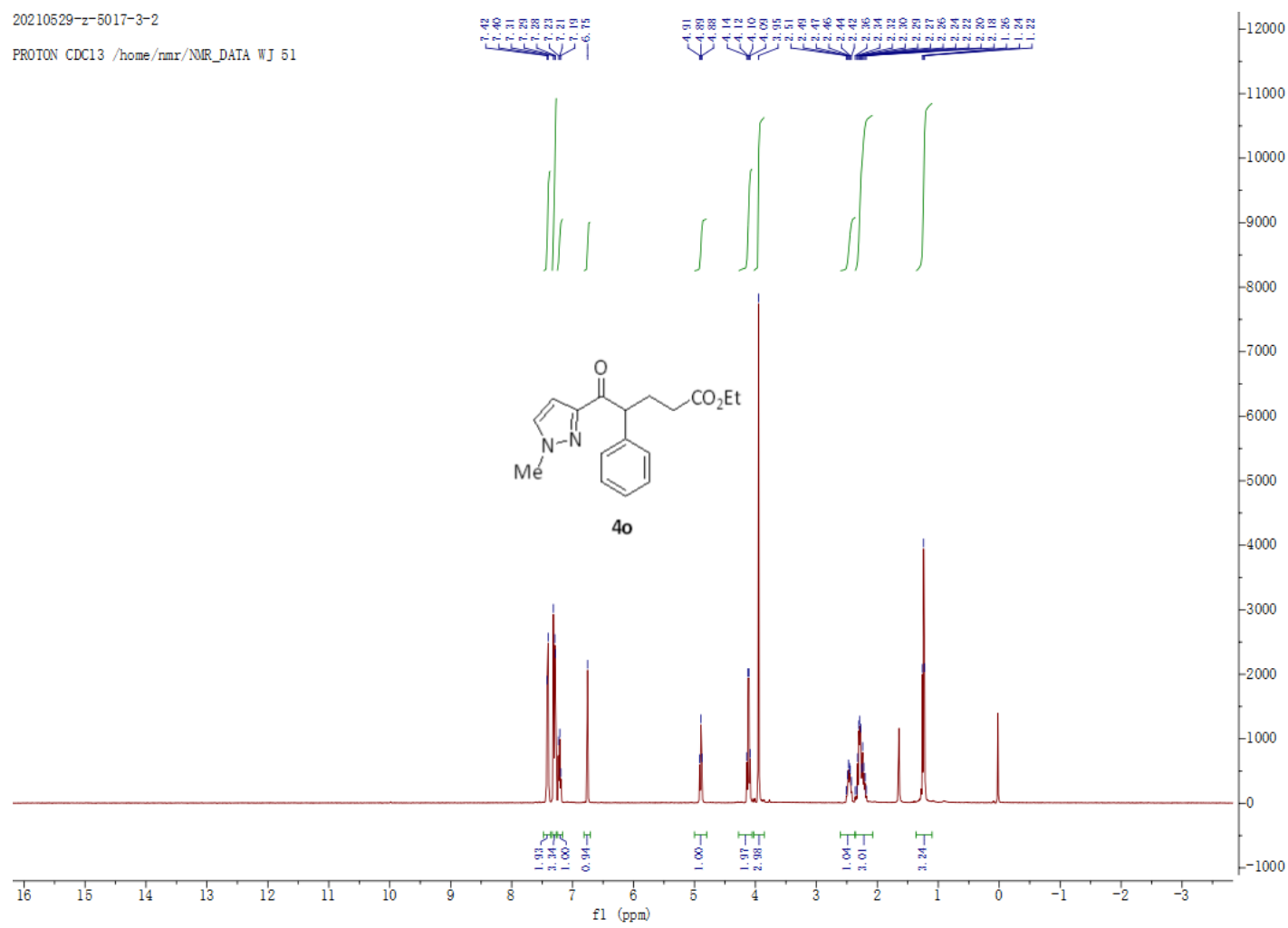

${ }^{13} \mathrm{C} \mathrm{NMR}\left(\mathrm{CDCl}_{3}, 100 \mathrm{MHz}\right)$

20210529-z-5017-3-2

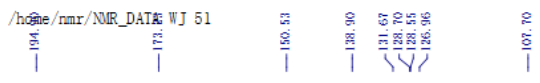
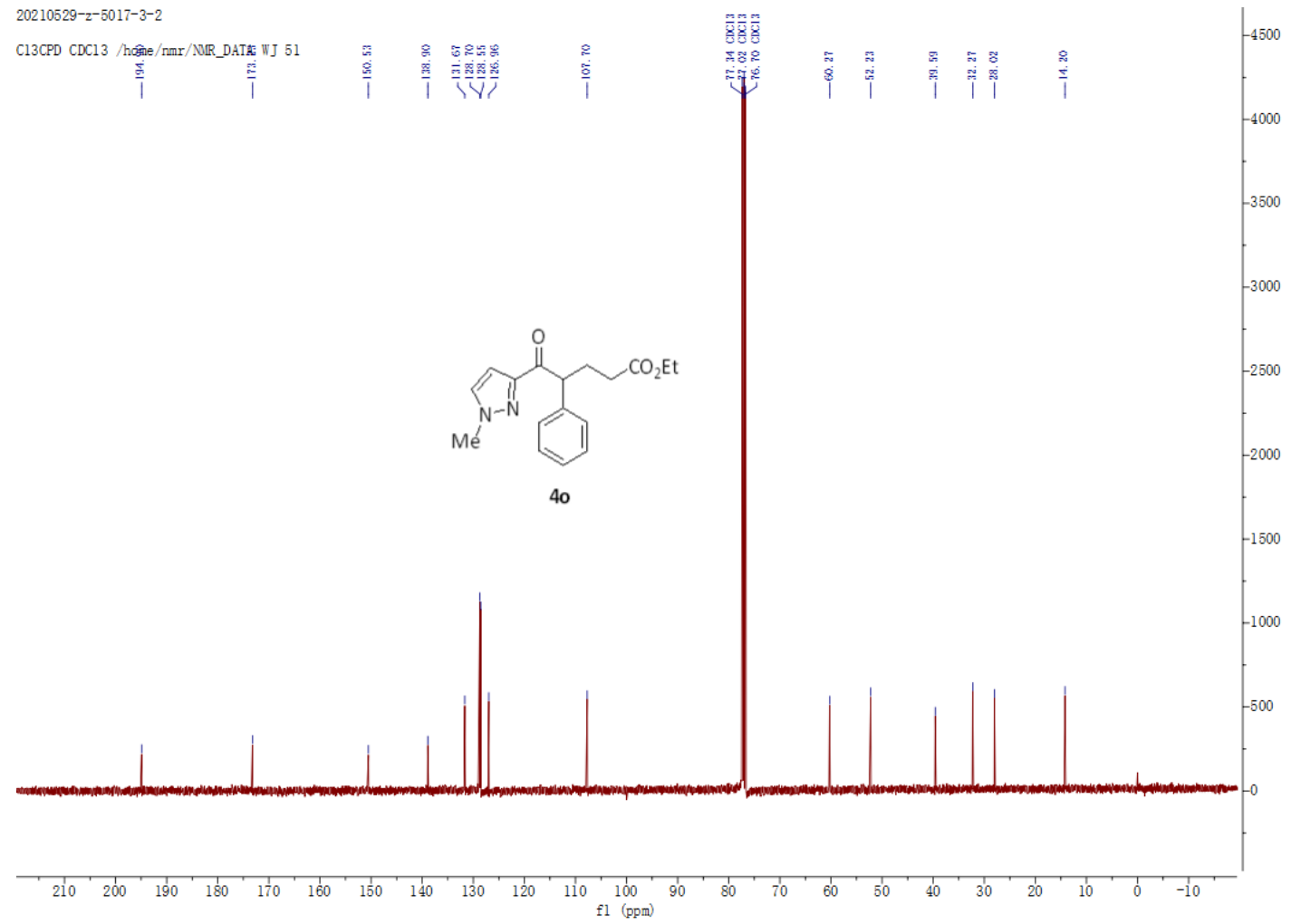


\section{Compound 4p}

${ }^{1} \mathrm{H} \mathrm{NMR}\left(\mathrm{CDCl}_{3}, 400 \mathrm{MHz}\right)$

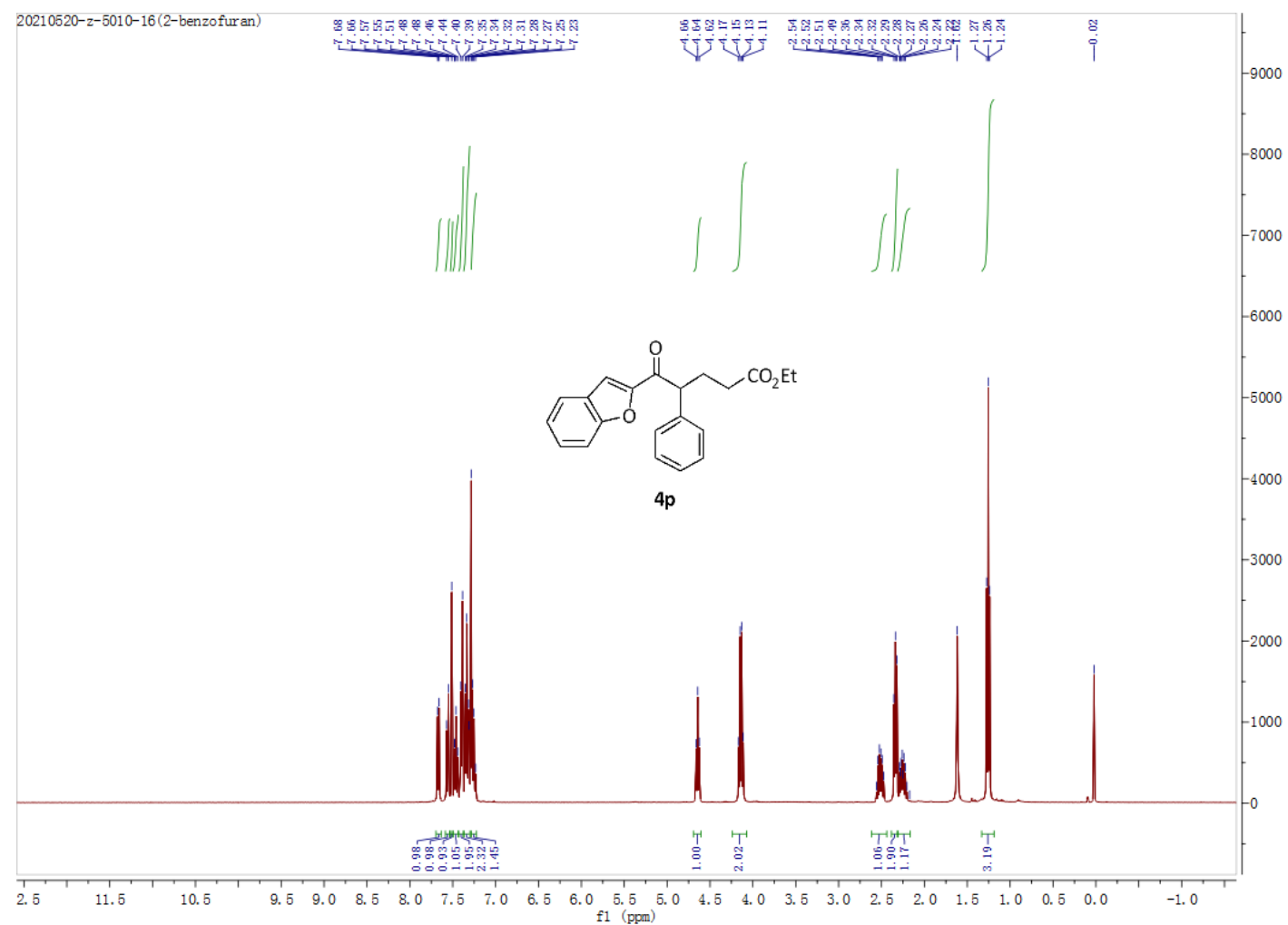

${ }^{13} \mathrm{C} \mathrm{NMR}\left(\mathrm{CDCl}_{3}, 100 \mathrm{MHz}\right)$

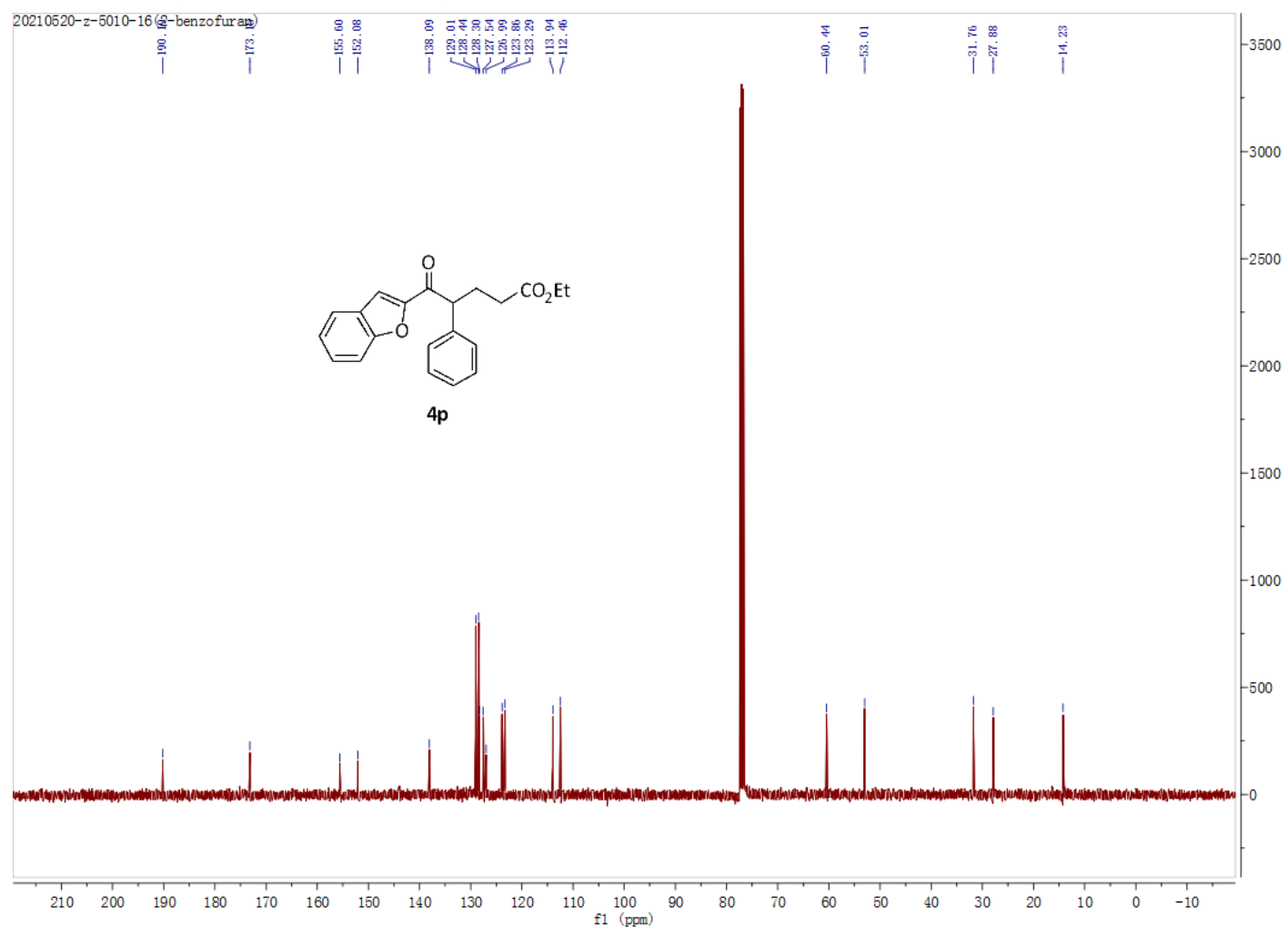




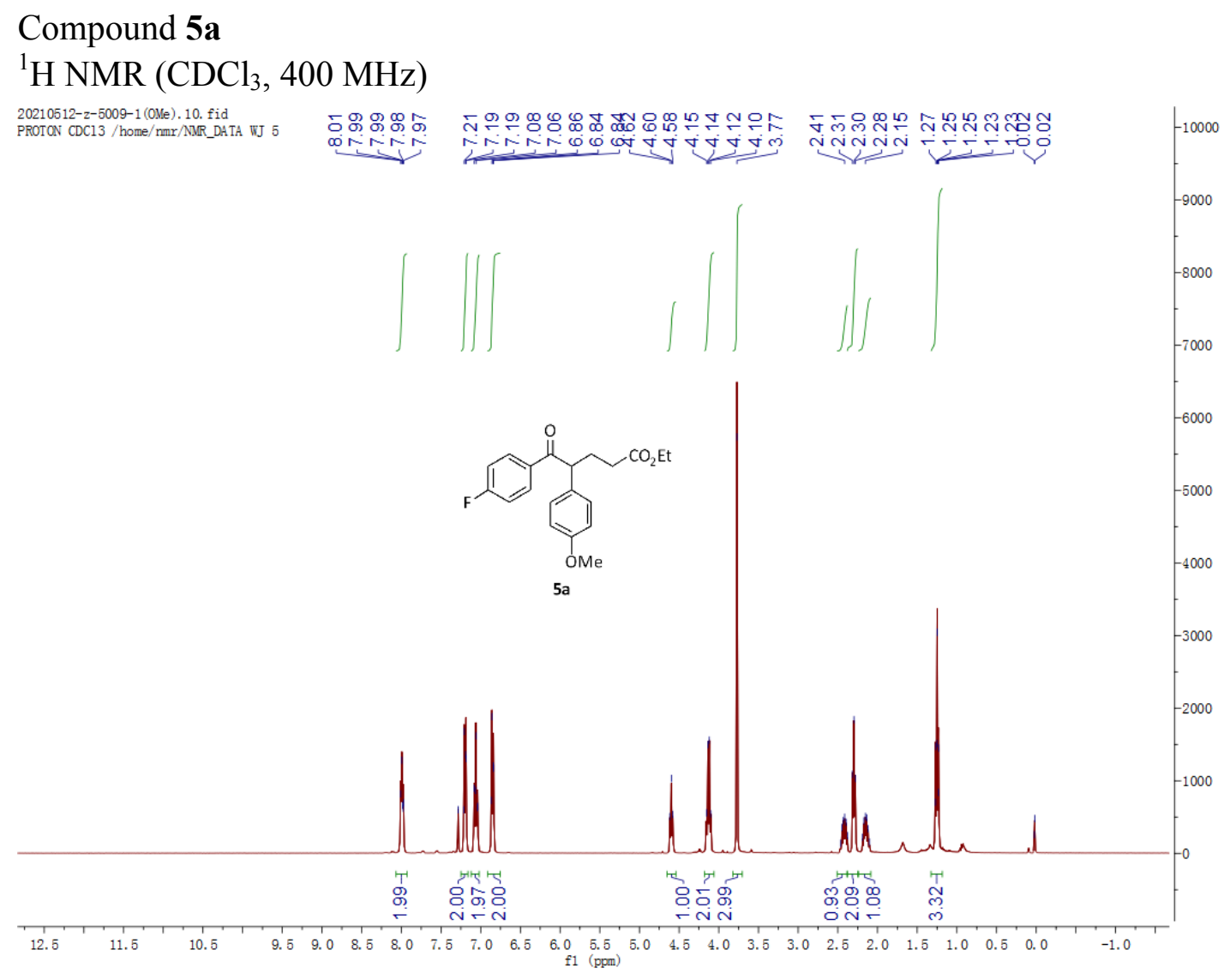

${ }^{19} \mathrm{~F} \mathrm{NMR}\left(\mathrm{CDCl}_{3}, 376 \mathrm{MHz}\right)$

20210512-z-5009-1 (0Me). 11. fid
F19CPD CDC13 /home/nmr/NMR_DATA WJ 5

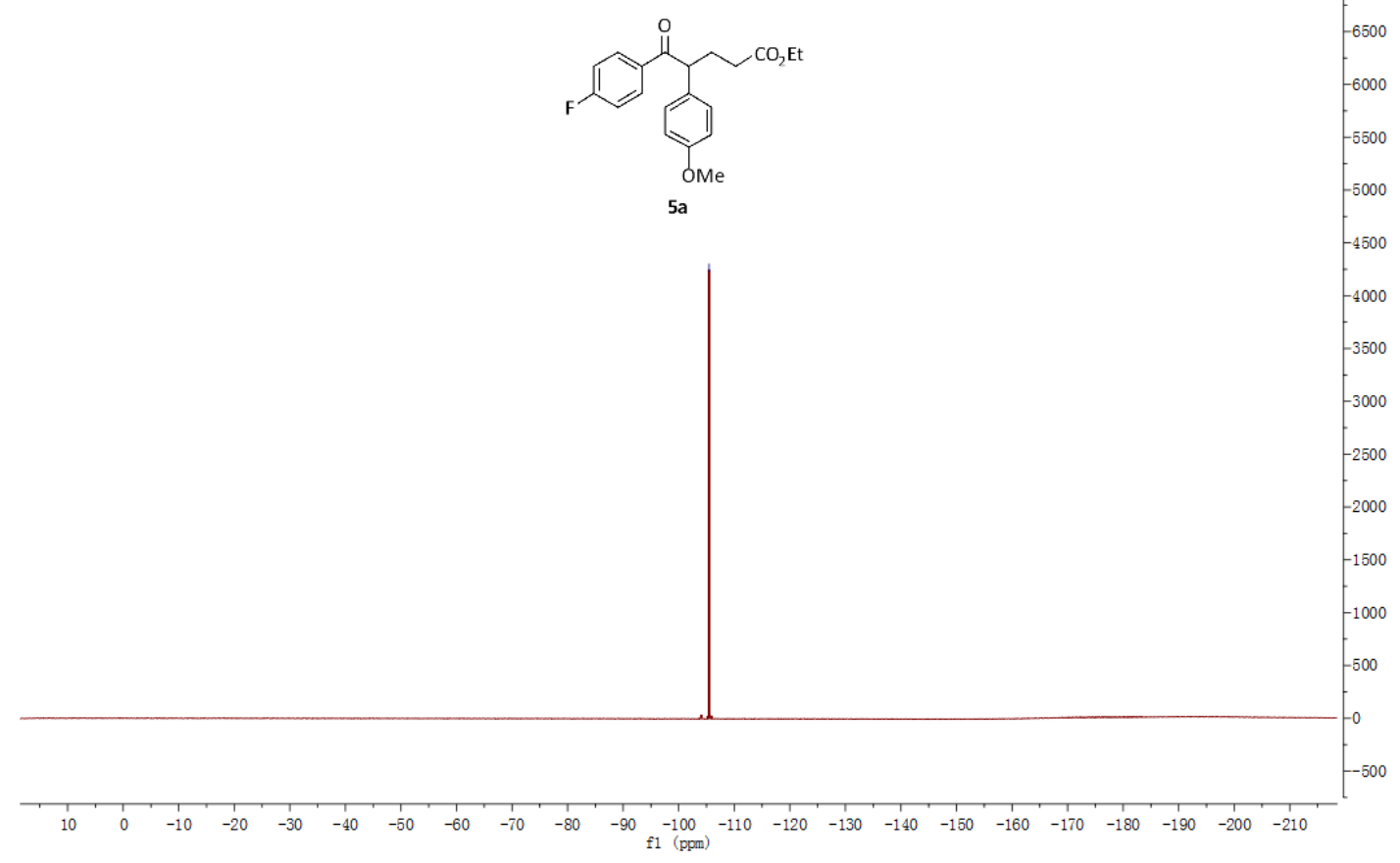


${ }^{13} \mathrm{C} \mathrm{NMR}\left(\mathrm{CDCl}_{3}, 100 \mathrm{MHz}\right)$

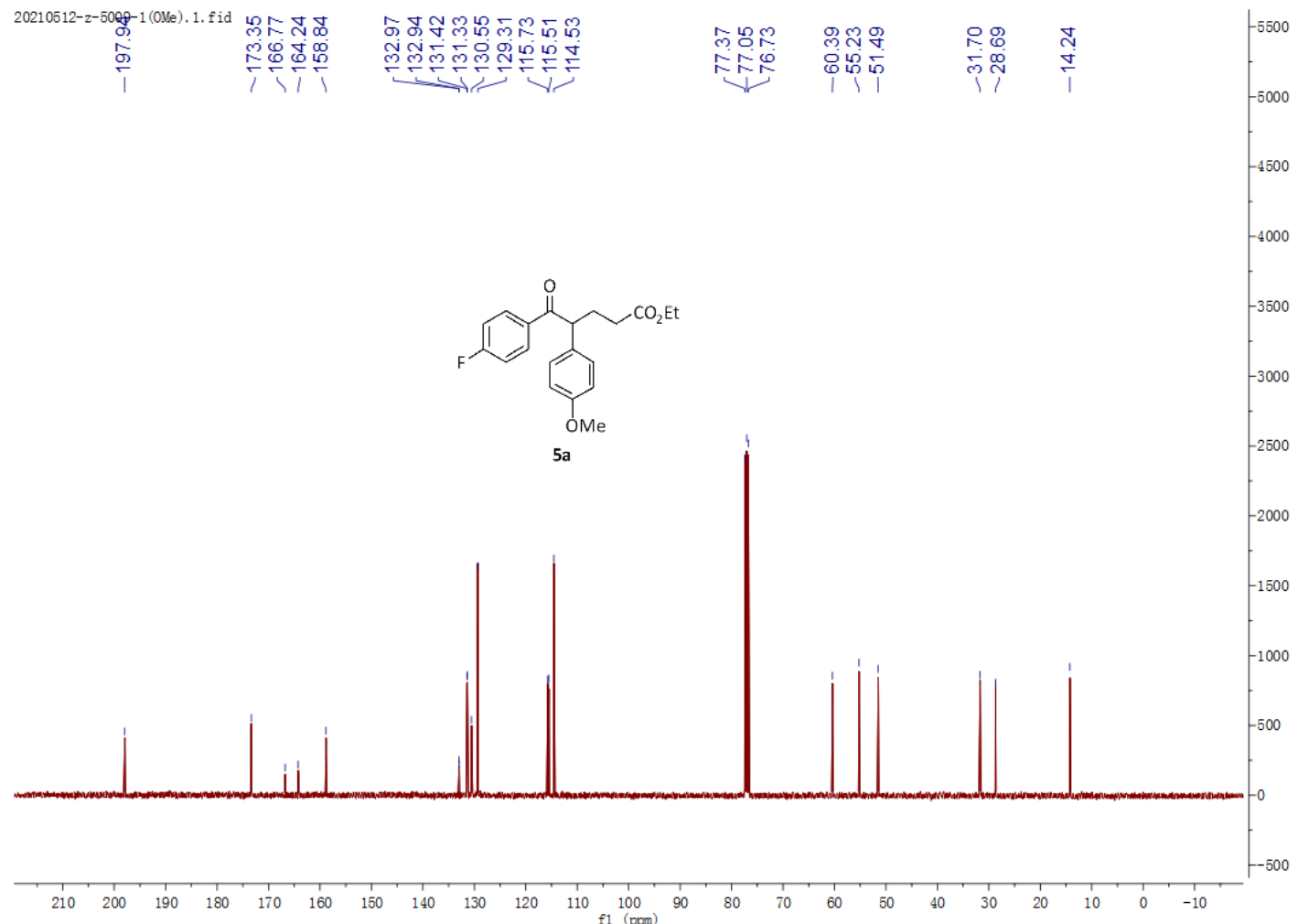




\section{Compound 5b}

${ }^{1} \mathrm{H} \mathrm{NMR}\left(\mathrm{CDCl}_{3}, 400 \mathrm{MHz}\right)$

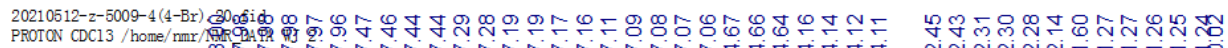

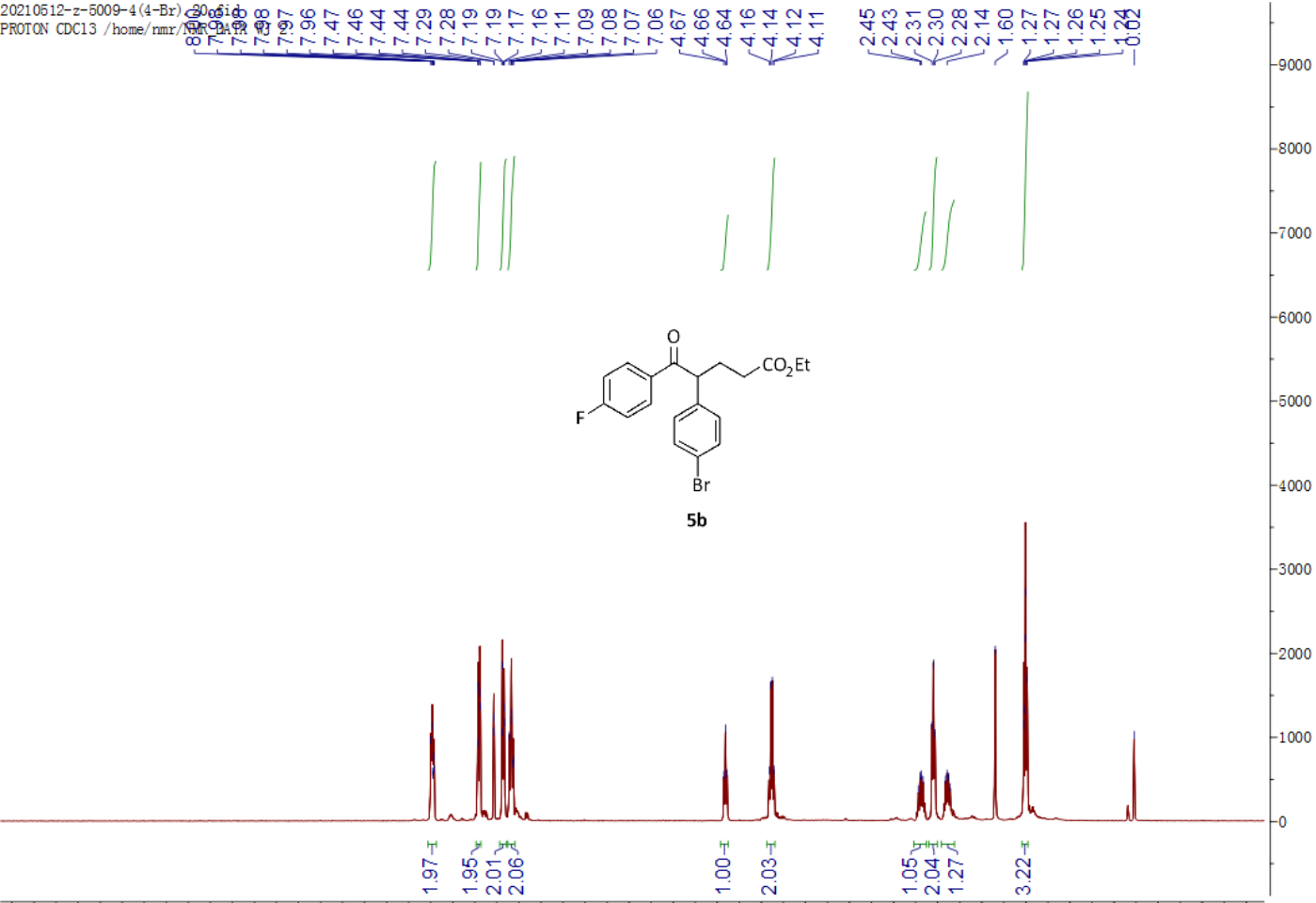

$\begin{array}{llllllllllllllllllllllllllllllllll}12.5 & 11.5 & 10.5 & 9.5 & 9.0 & 8.5 & 8.0 & 7.5 & 7.0 & 6.5 & 6.0 & 5.5 & 5.0 & 4.5 & 4.0 & 3.5 & 3.0 & 2.5 & 2.0 & 1.5 & 1.0 & 0.5 & 0.0 & -0.5\end{array}$

${ }^{19} \mathrm{~F} \mathrm{NMR}\left(\mathrm{CDCl}_{3}, 376 \mathrm{MHz}\right)$

20210512-z-5009-4(4-Br). 11. fid
F19CPD CDC13/home/nmr/NMR_DATA WJ 3

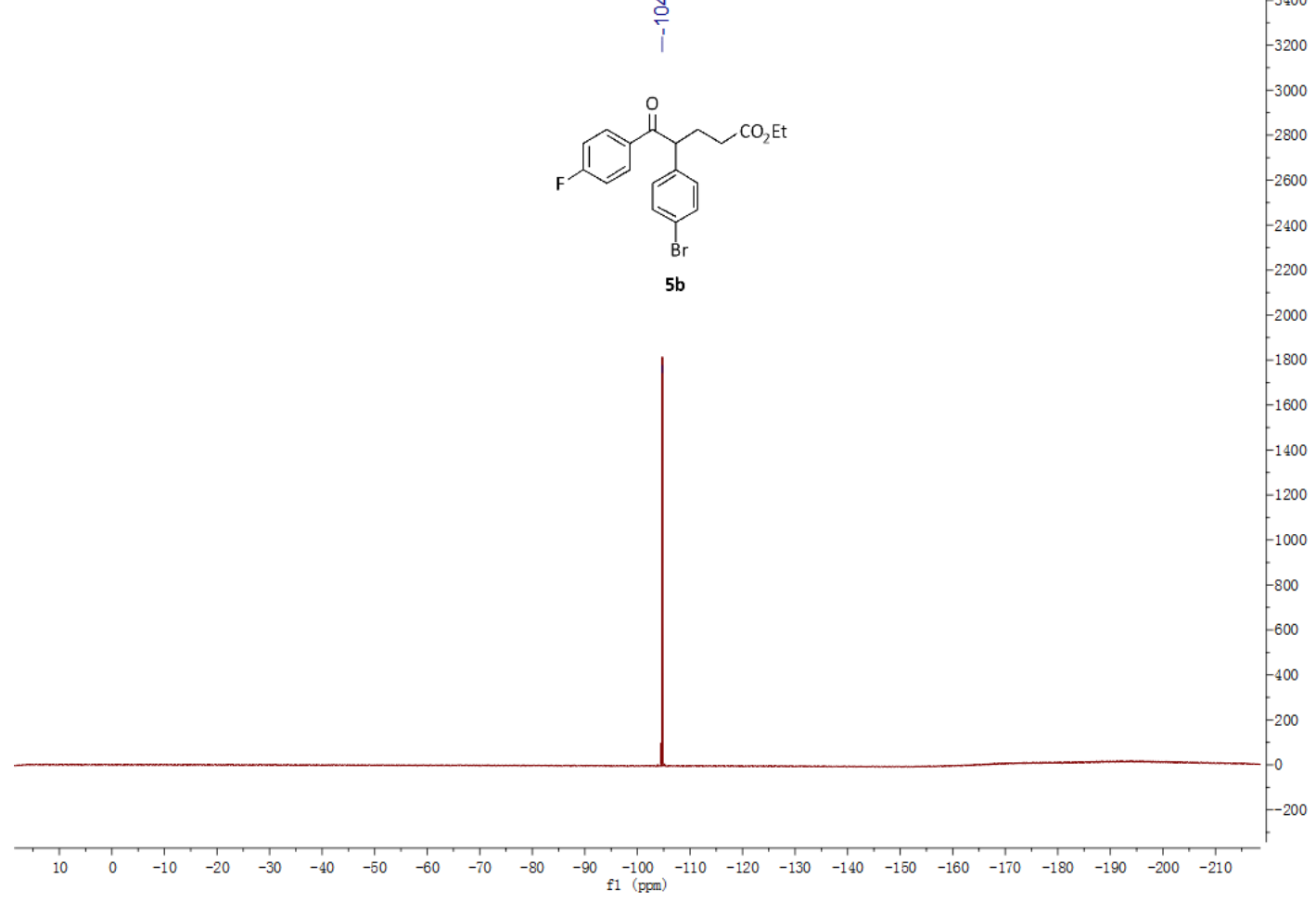


${ }^{13} \mathrm{C} \mathrm{NMR}\left(\mathrm{CDCl}_{3}, 100 \mathrm{MHz}\right)$

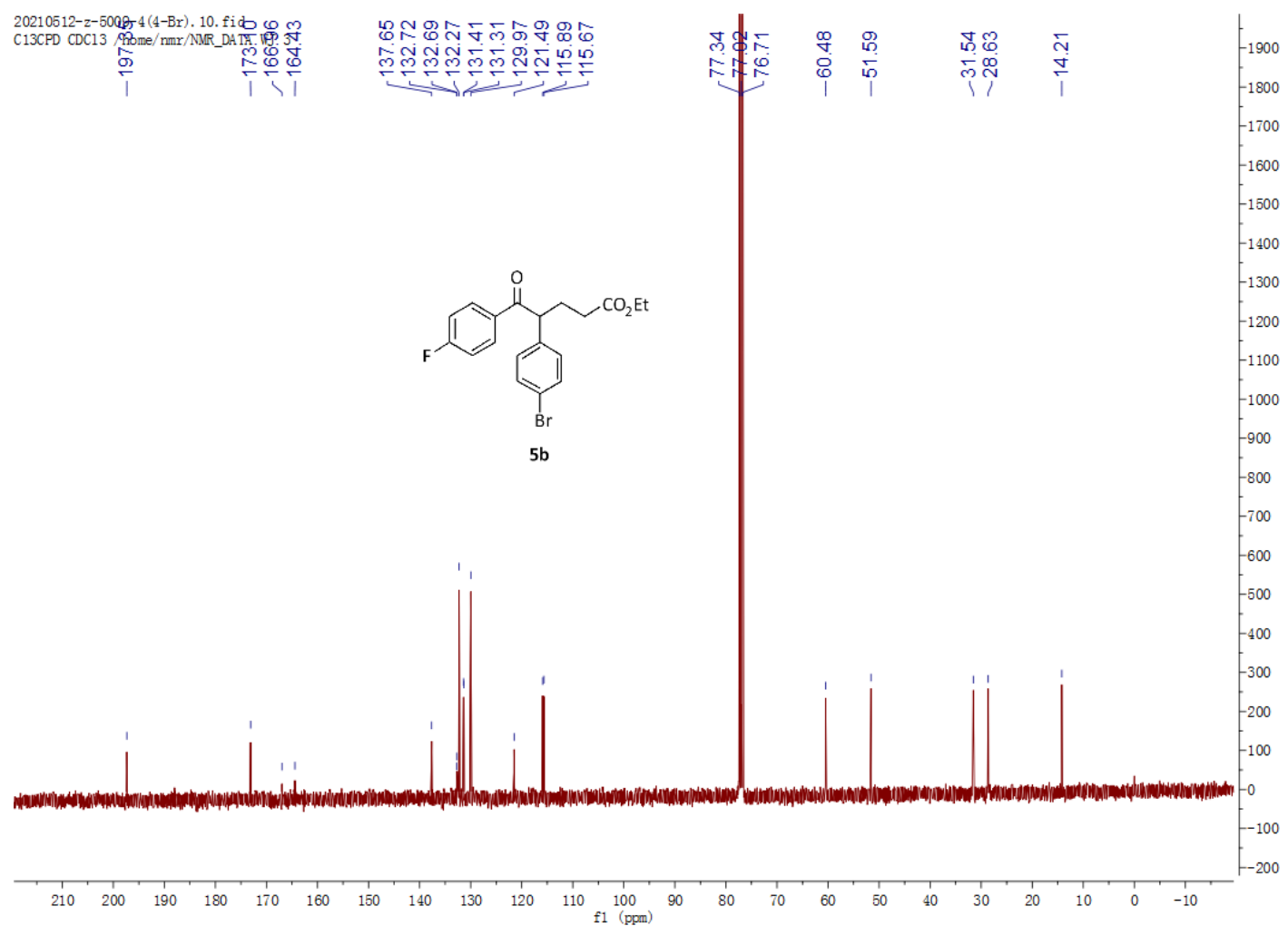




\section{Compound 5c}

${ }^{1} \mathrm{H} \mathrm{NMR}\left(\mathrm{CDCl}_{3}, 400 \mathrm{MHz}\right)$

20210513-z-5009-3(4-CN). 10. fid
PROTON CDC13 /home/nmr/NMR_DATA WJ 1

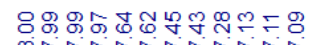

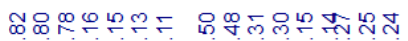

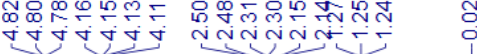

이ำ
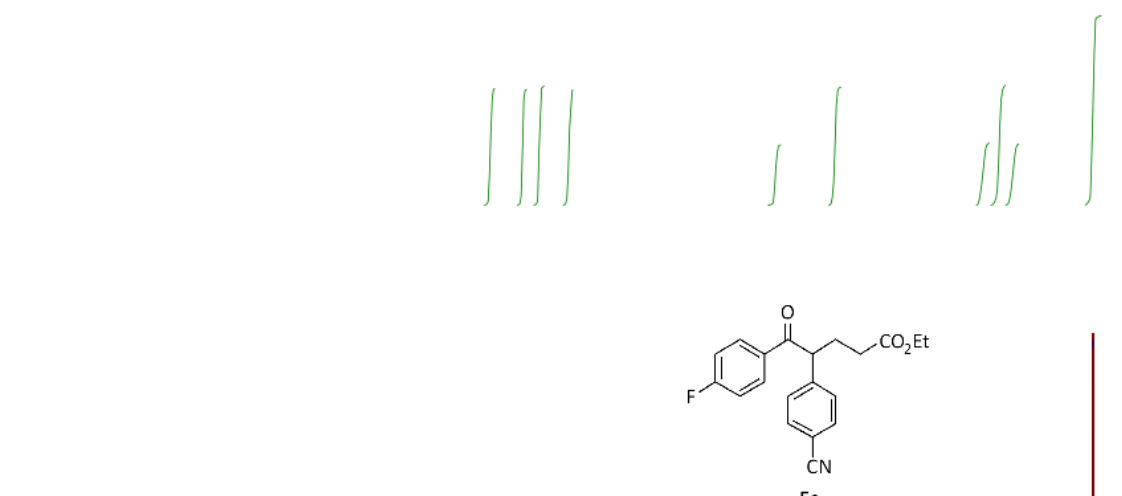

$-9000$

$5 c$

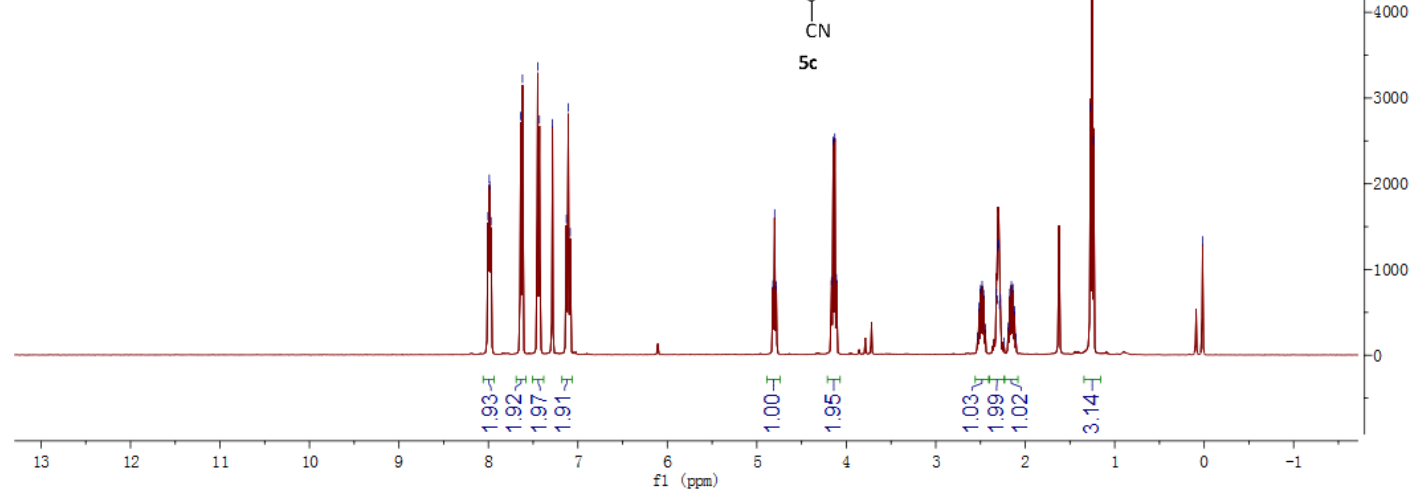

${ }^{19} \mathrm{~F}$ NMR $\left(\mathrm{CDCl}_{3}, 376 \mathrm{MHz}\right)$

20210513-z-5009-3(4-CN). 11. fid
F19CPD CDC13 /home/nmr/NMR_DATA WJ 1

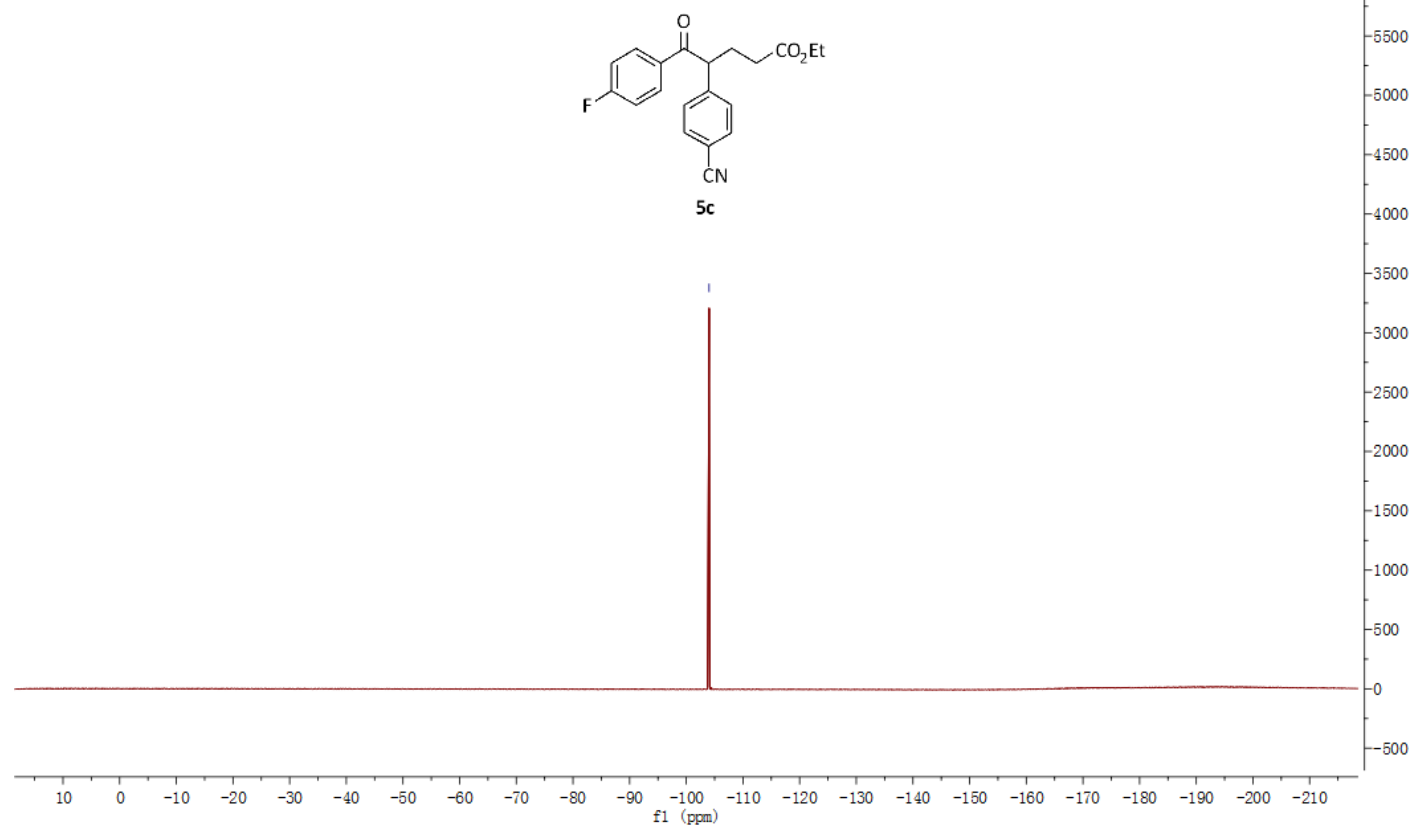


${ }^{13} \mathrm{C} \mathrm{NMR}\left(\mathrm{CDCl}_{3}, 100 \mathrm{MHz}\right)$

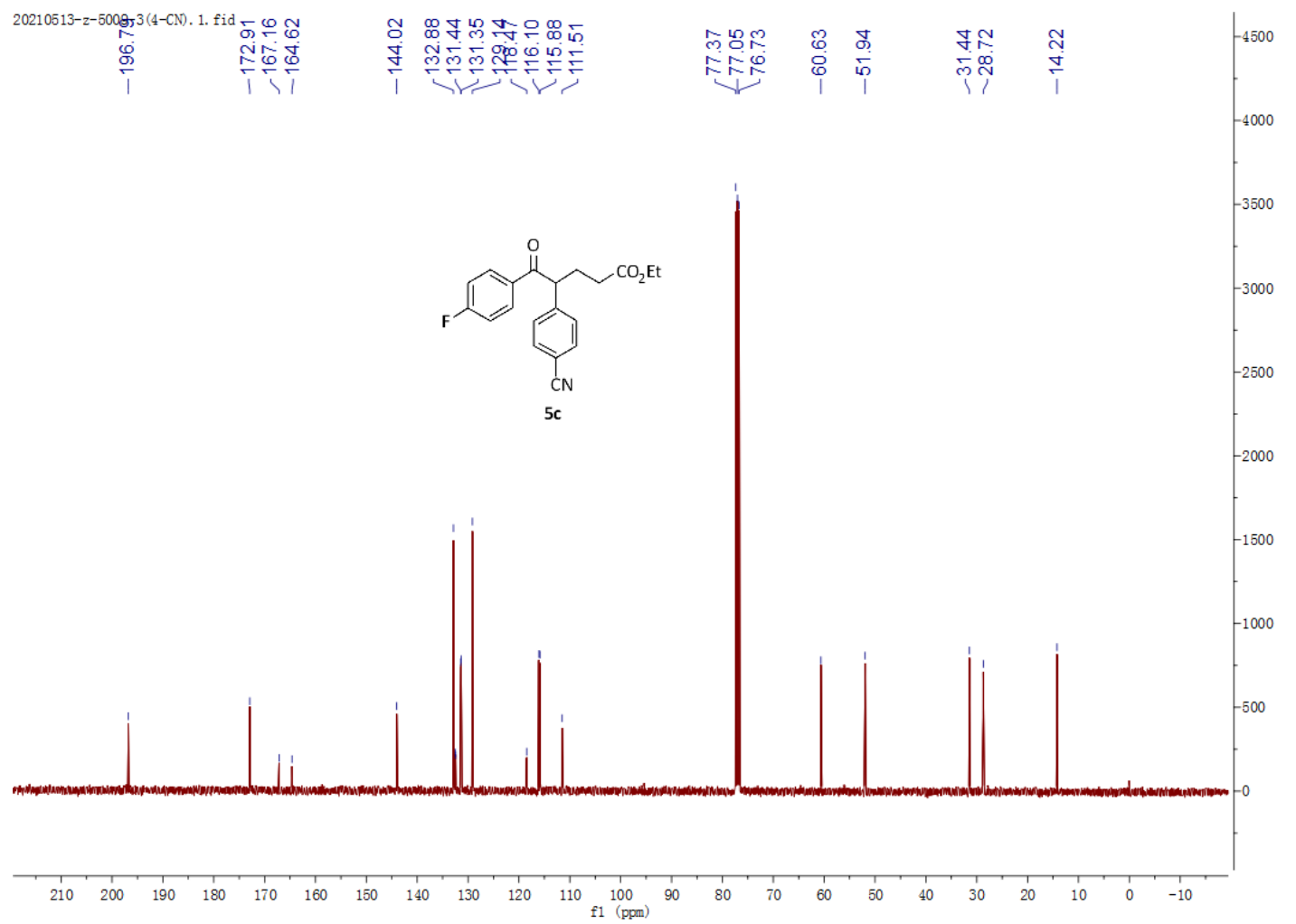




\section{Compound 5d}

${ }^{1} \mathrm{H} \mathrm{NMR}\left(\mathrm{CDCl}_{3}, 400 \mathrm{MHz}\right)$

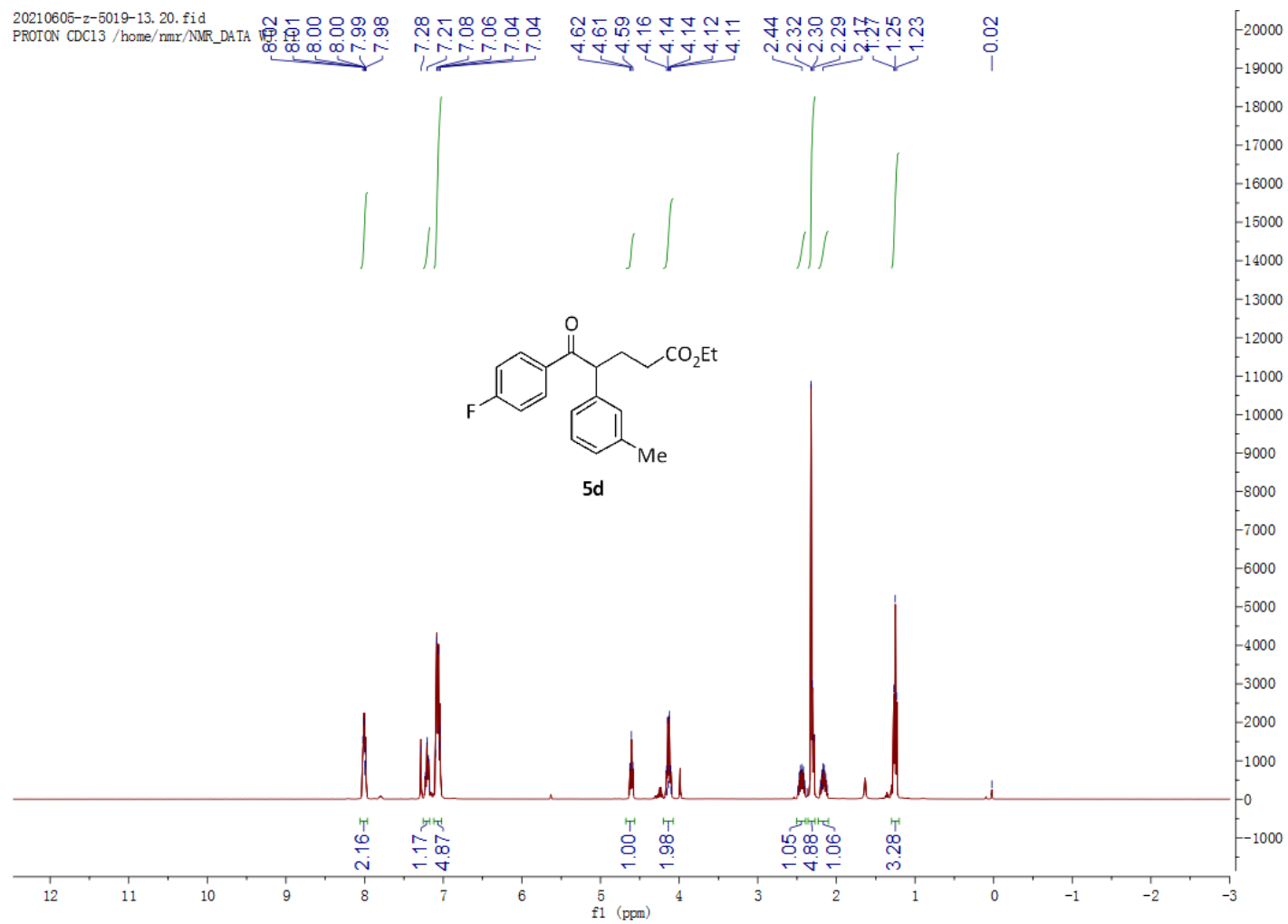

${ }^{19} \mathrm{~F} \mathrm{NMR}\left(\mathrm{CDCl}_{3}, 376 \mathrm{MHz}\right)$

20210605-z-5019-13. 21. fid
F19CPD CDC13 /home/nmr/NMR_DATA WJ 11

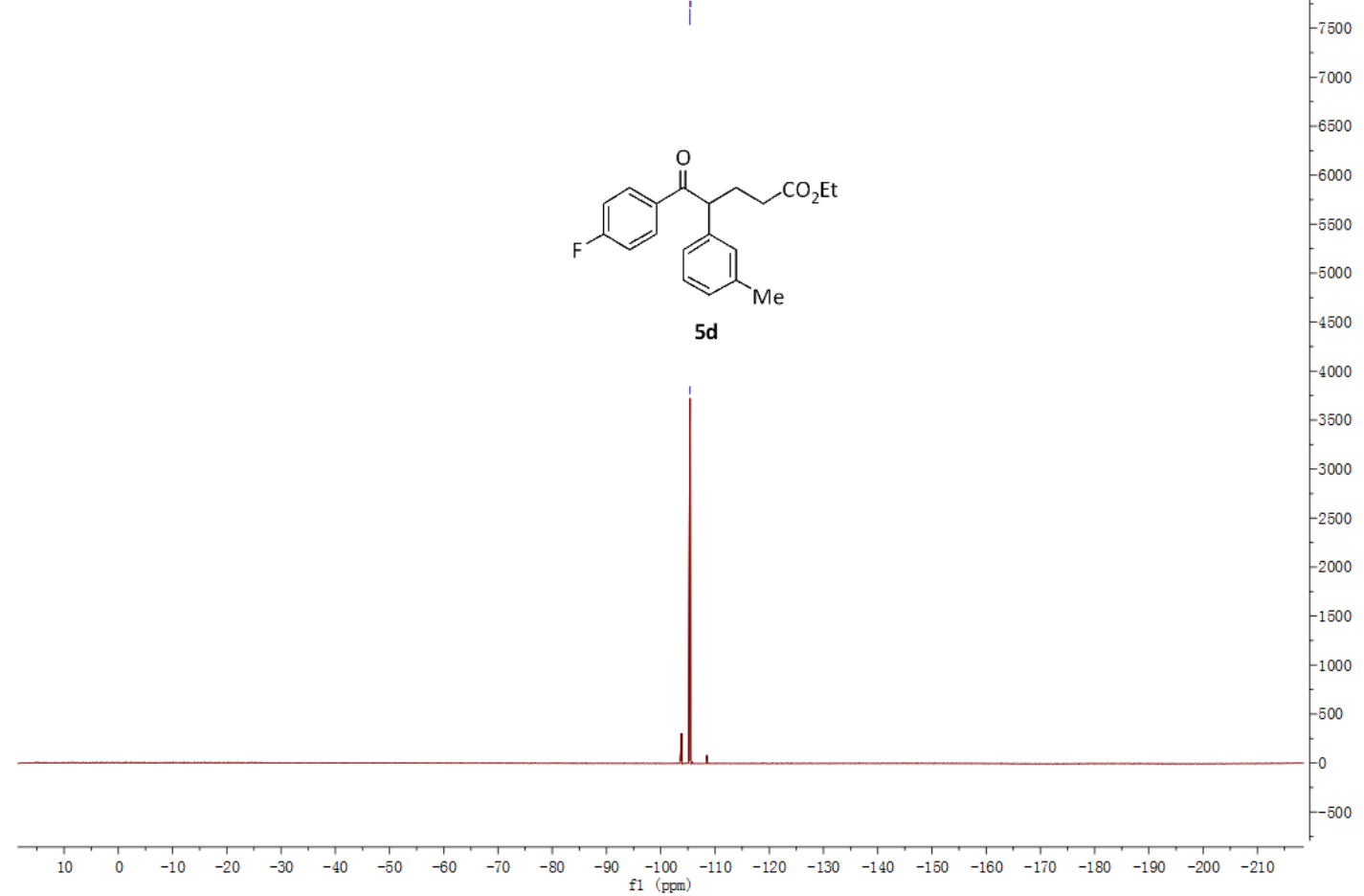


${ }^{13} \mathrm{C} \mathrm{NMR}\left(\mathrm{CDCl}_{3}, 100 \mathrm{MHz}\right)$

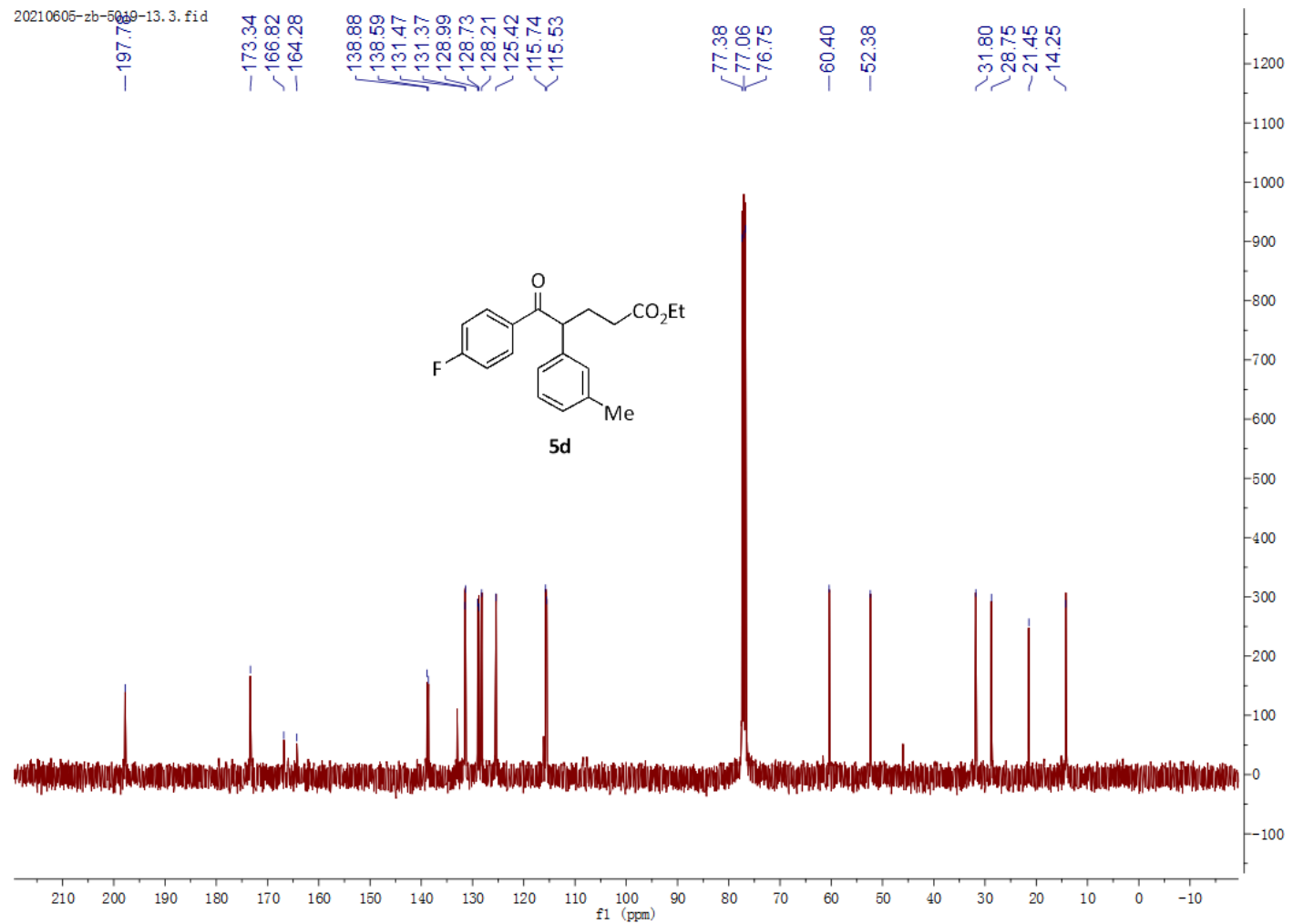




\section{Compound $\mathbf{5 e}$}

${ }^{1} \mathrm{H} \mathrm{NMR}\left(\mathrm{CDCl}_{3}, 400 \mathrm{MHz}\right)$

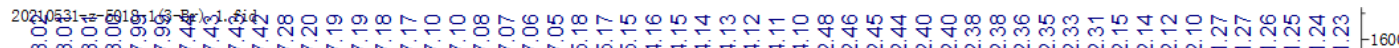

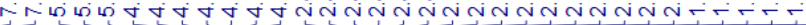

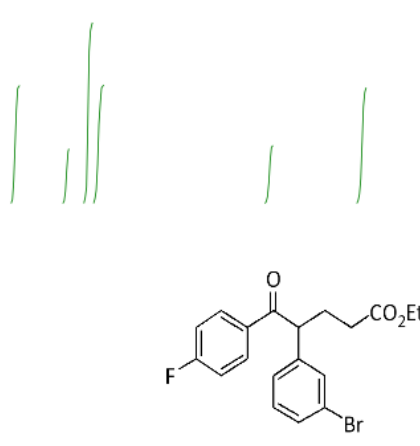

5 e

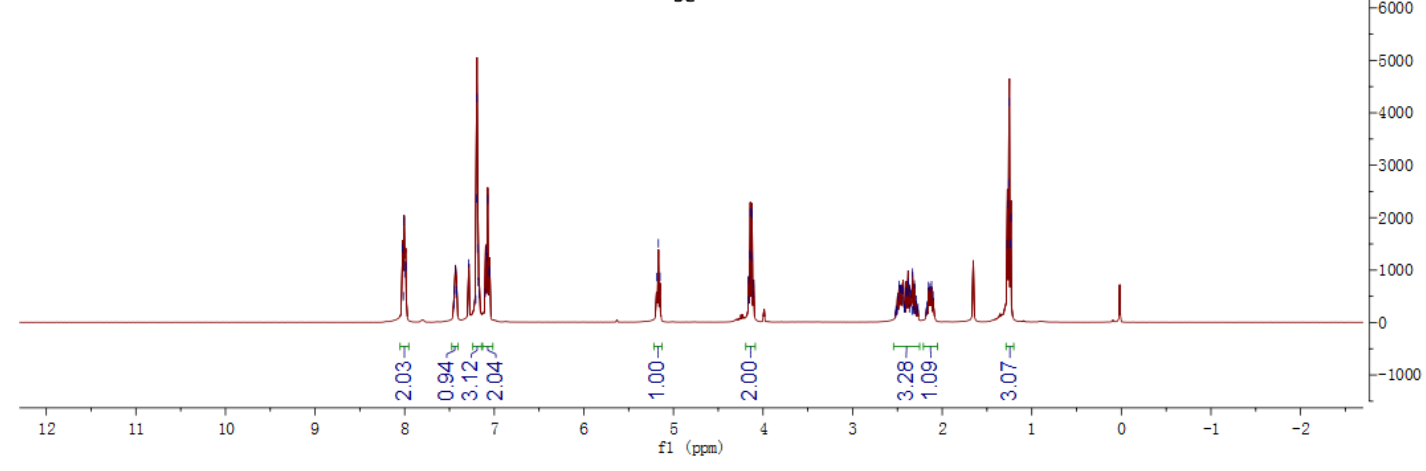

${ }^{19} \mathrm{~F}$ NMR $\left(\mathrm{CDCl}_{3}, 376 \mathrm{MHz}\right)$

$20210531-z-5018-1(3-B r)$. 2, fid
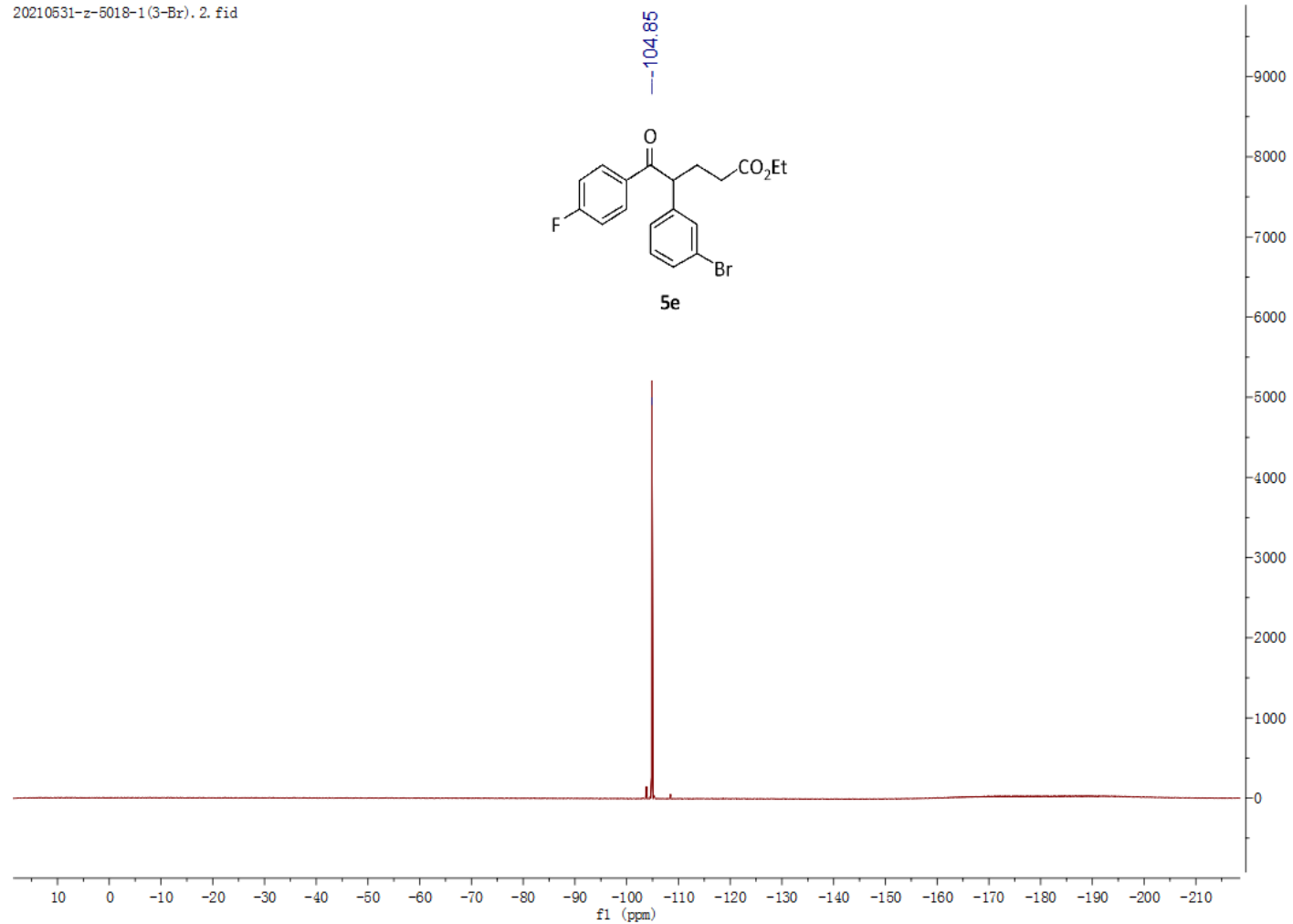
${ }^{13} \mathrm{C} \mathrm{NMR}\left(\mathrm{CDCl}_{3}, 100 \mathrm{MHz}\right)$

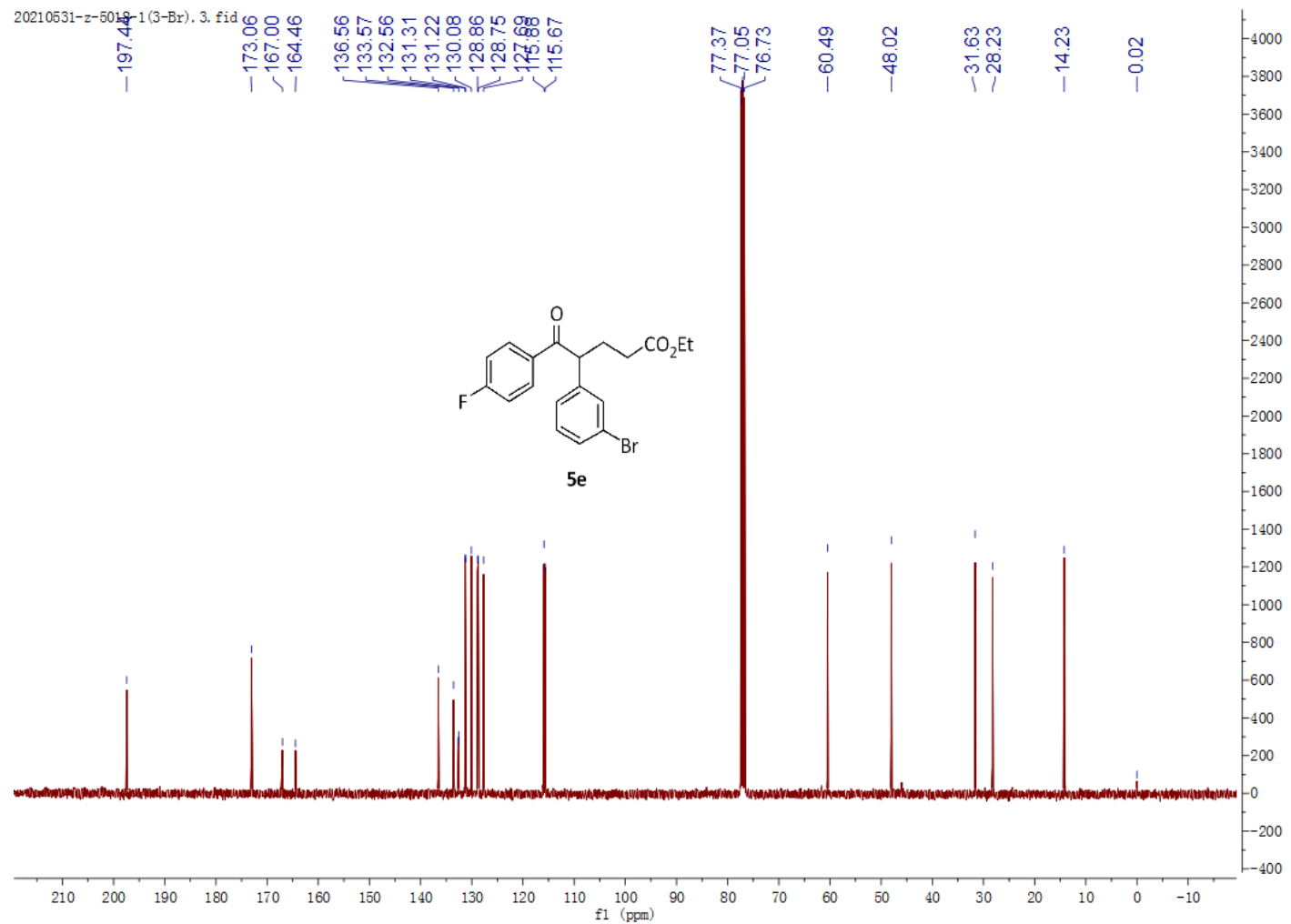




\section{Compound $\mathbf{5 f}$}

${ }^{1} \mathrm{H} \mathrm{NMR}\left(\mathrm{CDCl}_{3}, 400 \mathrm{MHz}\right)$

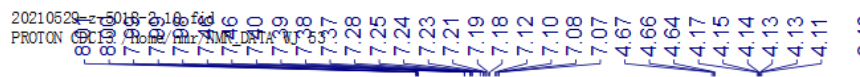

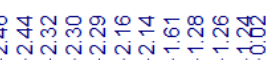

$-11000$

\|\|
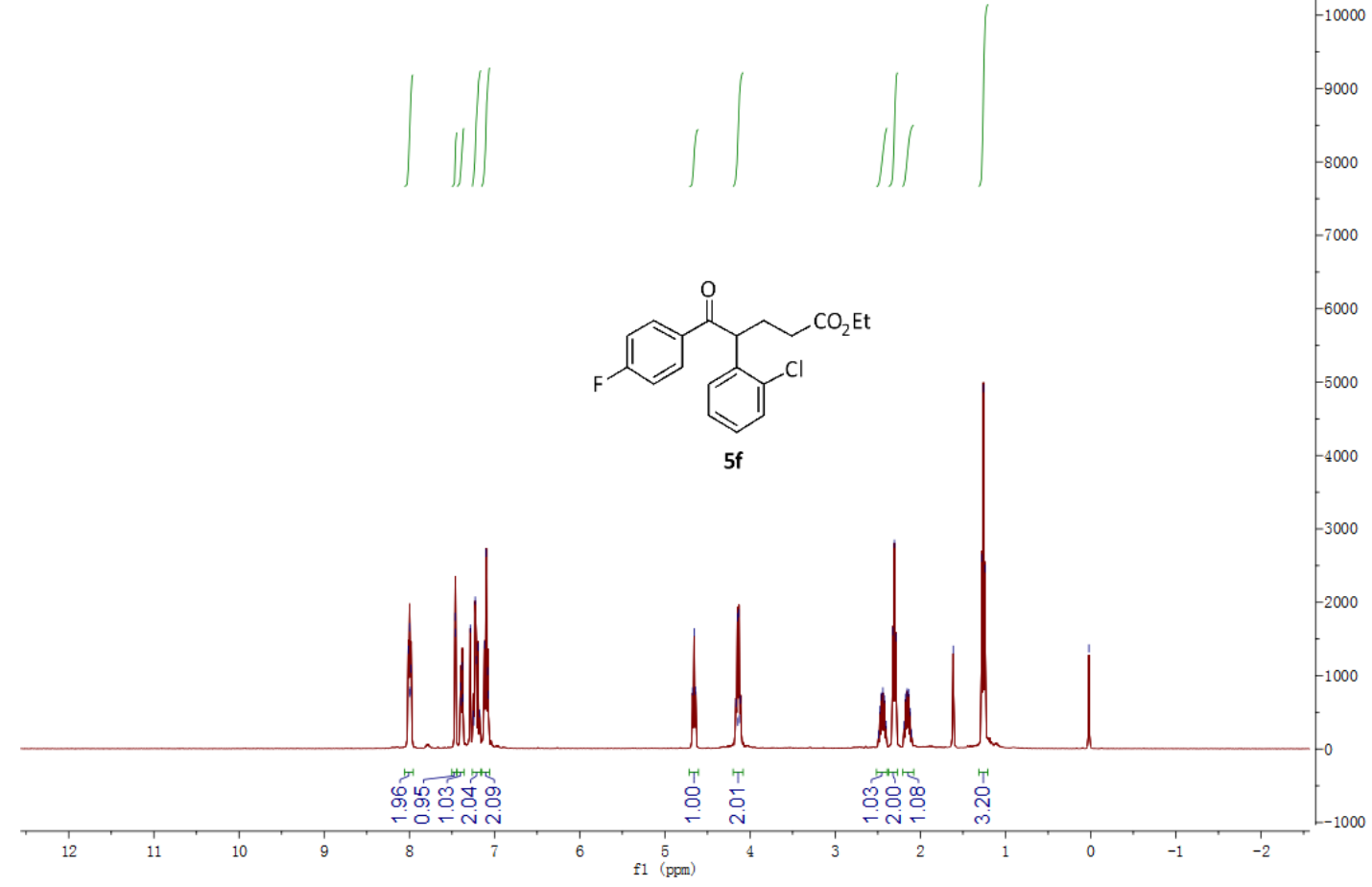

10000

${ }^{19} \mathrm{~F} \mathrm{NMR}\left(\mathrm{CDCl}_{3}, 376 \mathrm{MHz}\right)$

20210529-z-5018-2.11. fid
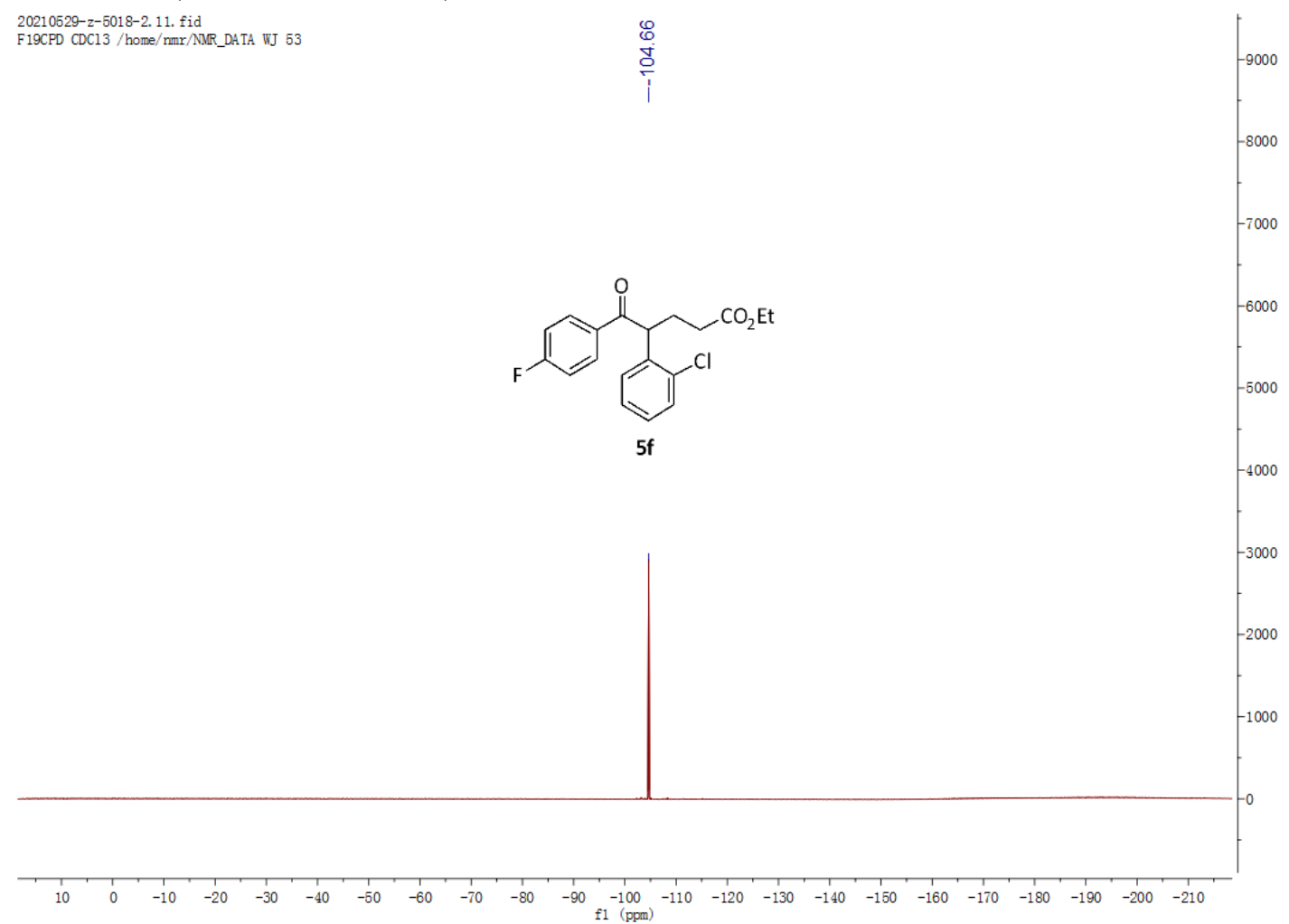

$-7000$ 
${ }^{13} \mathrm{C} \mathrm{NMR}\left(\mathrm{CDCl}_{3}, 100 \mathrm{MHz}\right)$

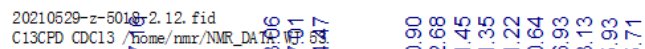

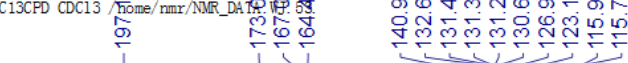

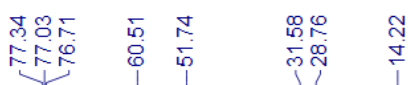

$-2500$<smiles>CC(C)(C)OC(=O)CC(C(=O)c1ccc(F)cc1)c1ccccc1Cl</smiles>

$-1000$

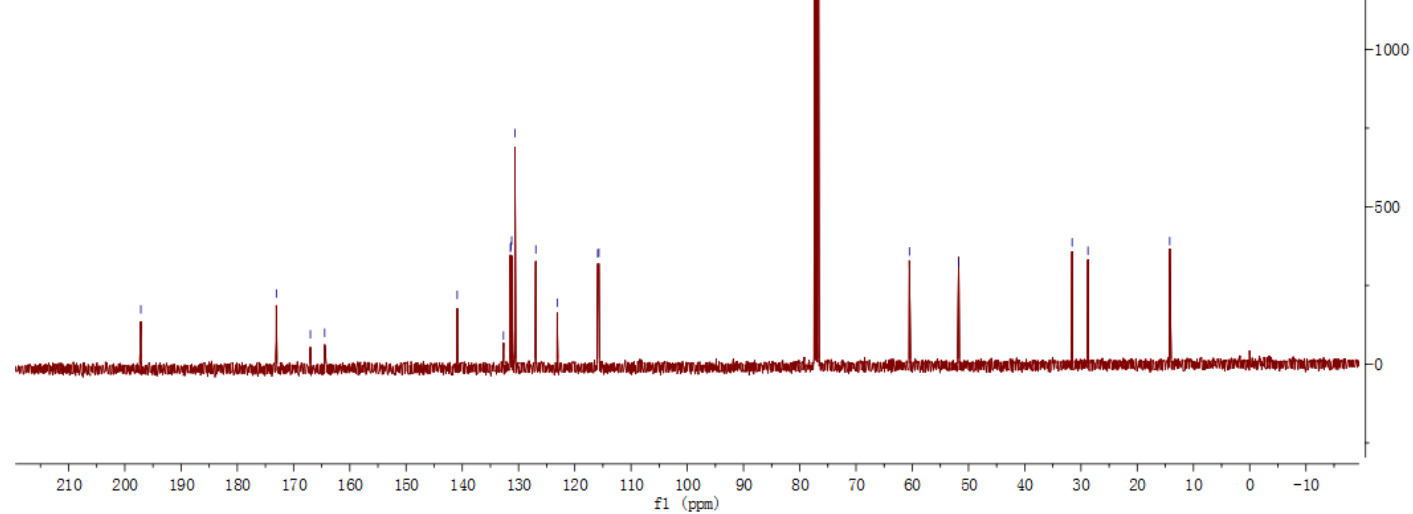




\section{Compound 5g}

${ }^{1} \mathrm{H} \mathrm{NMR}\left(\mathrm{CDCl}_{3}, 400 \mathrm{MHz}\right)$

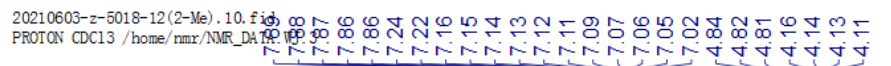

nivivi

$-18000$

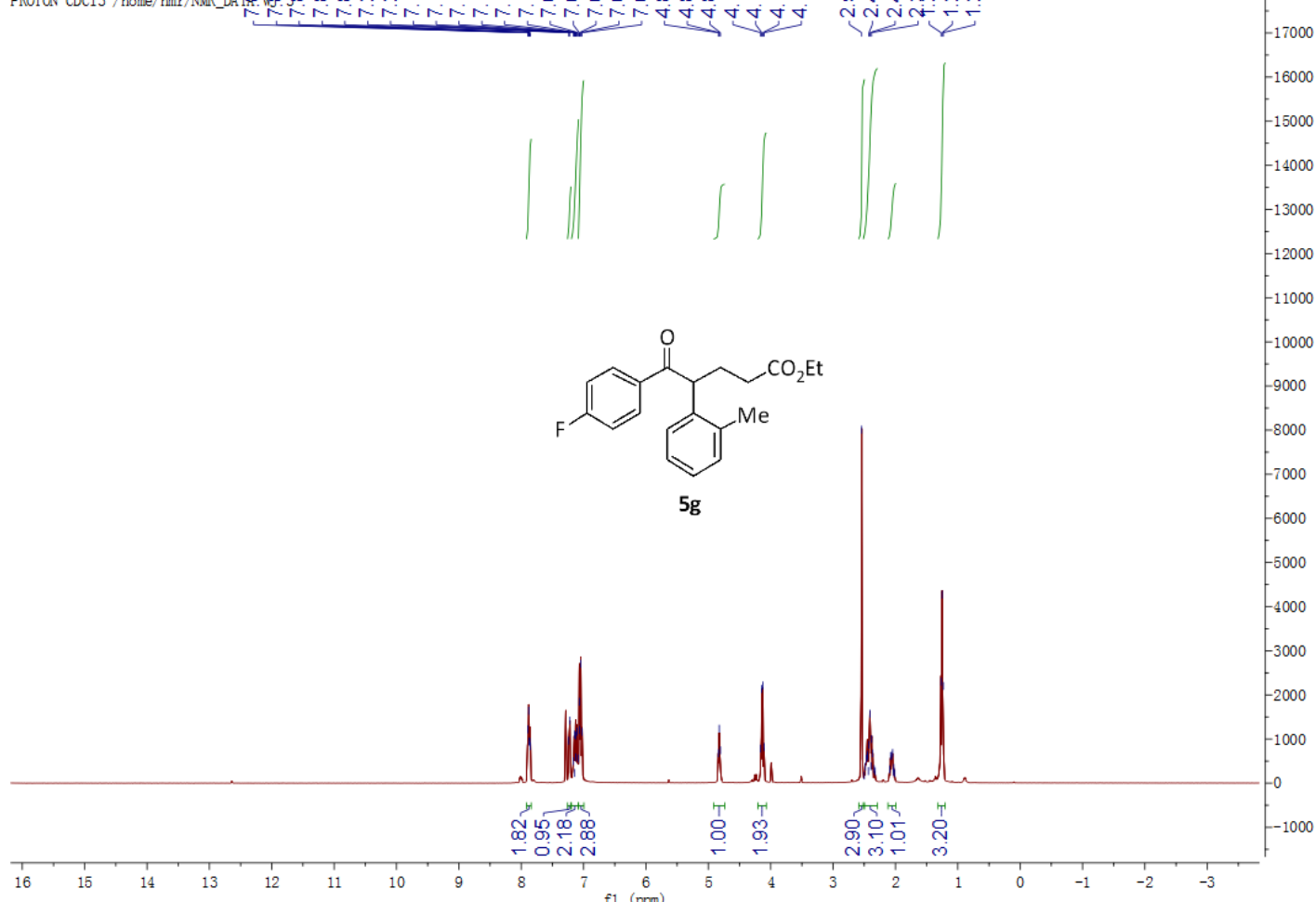

${ }^{19} \mathrm{~F} \mathrm{NMR}\left(\mathrm{CDCl}_{3}, 376 \mathrm{MHz}\right)$

20210603-z-5018-12(2-Me).11.fid
F19CPD CDC13 /home/nmr/NMR_DATA WJ 3

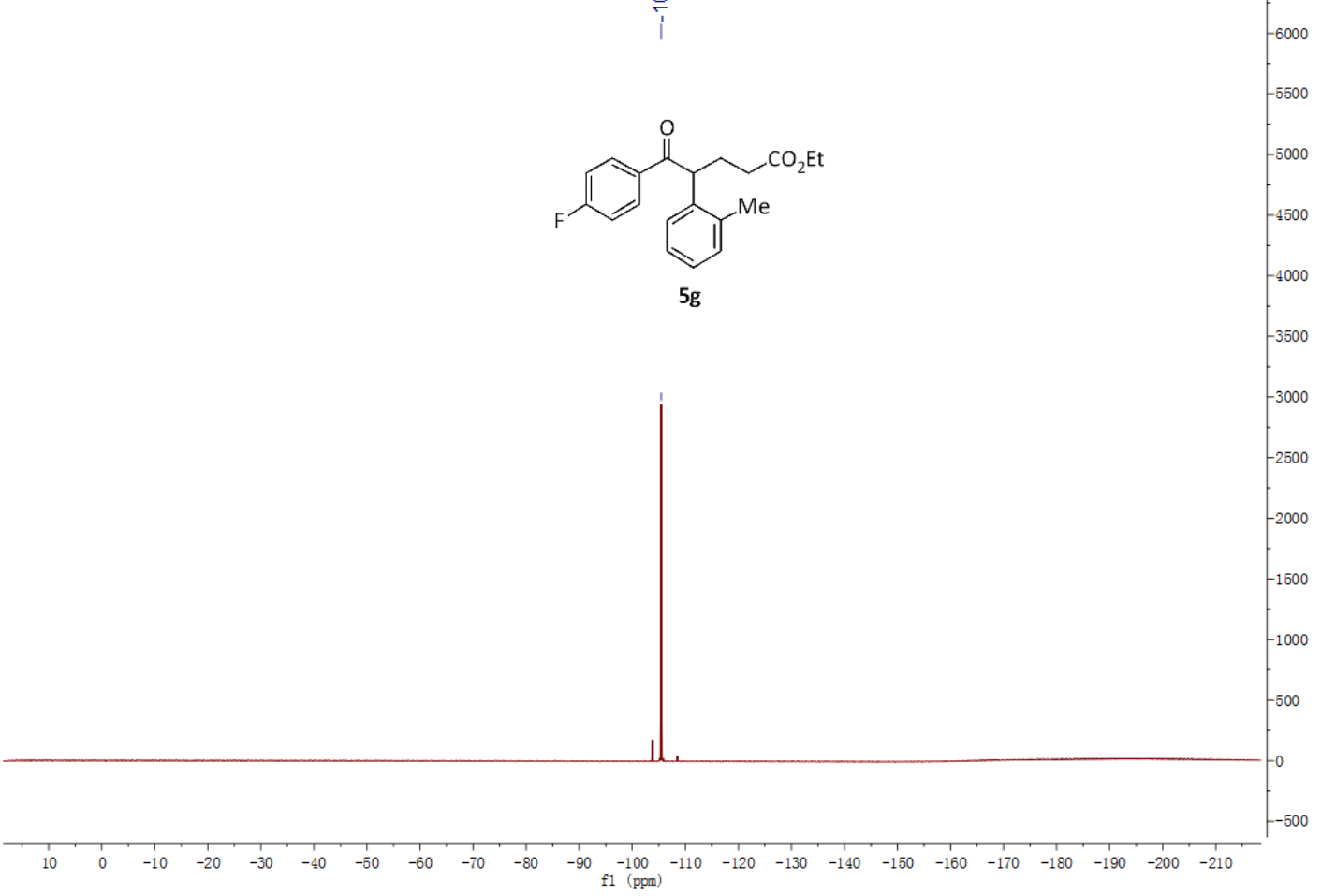


${ }^{13} \mathrm{C} \mathrm{NMR}\left(\mathrm{CDCl}_{3}, 100 \mathrm{MHz}\right)$

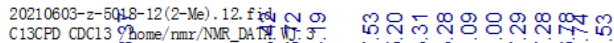

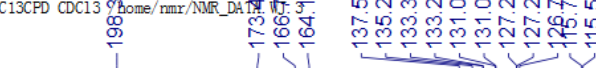

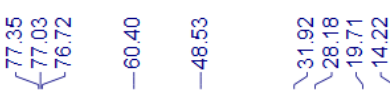

$-4000$

$-3500$

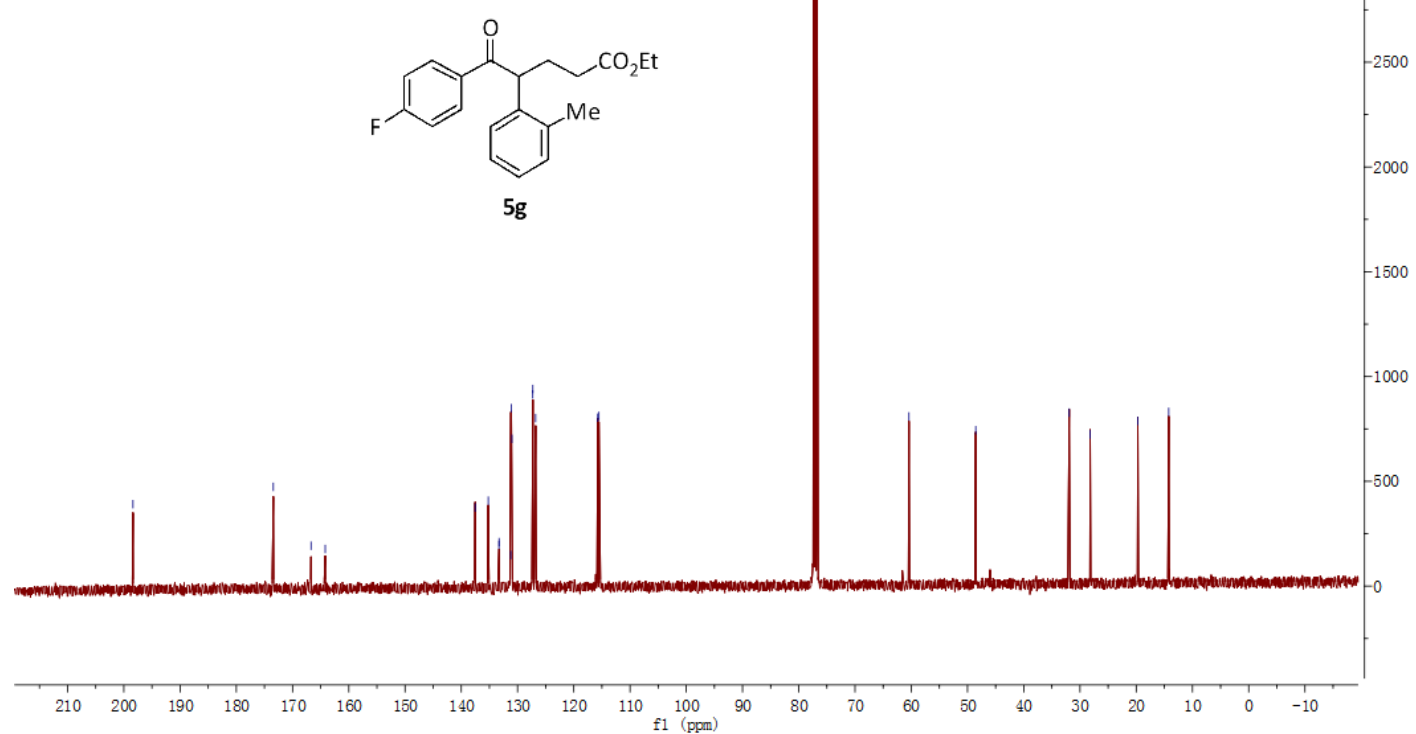

$-2500$ 


\section{Compound $\mathbf{5 h}$}

${ }^{1} \mathrm{H} \mathrm{NMR}\left(\mathrm{CDCl}_{3}, 400 \mathrm{MHz}\right)$

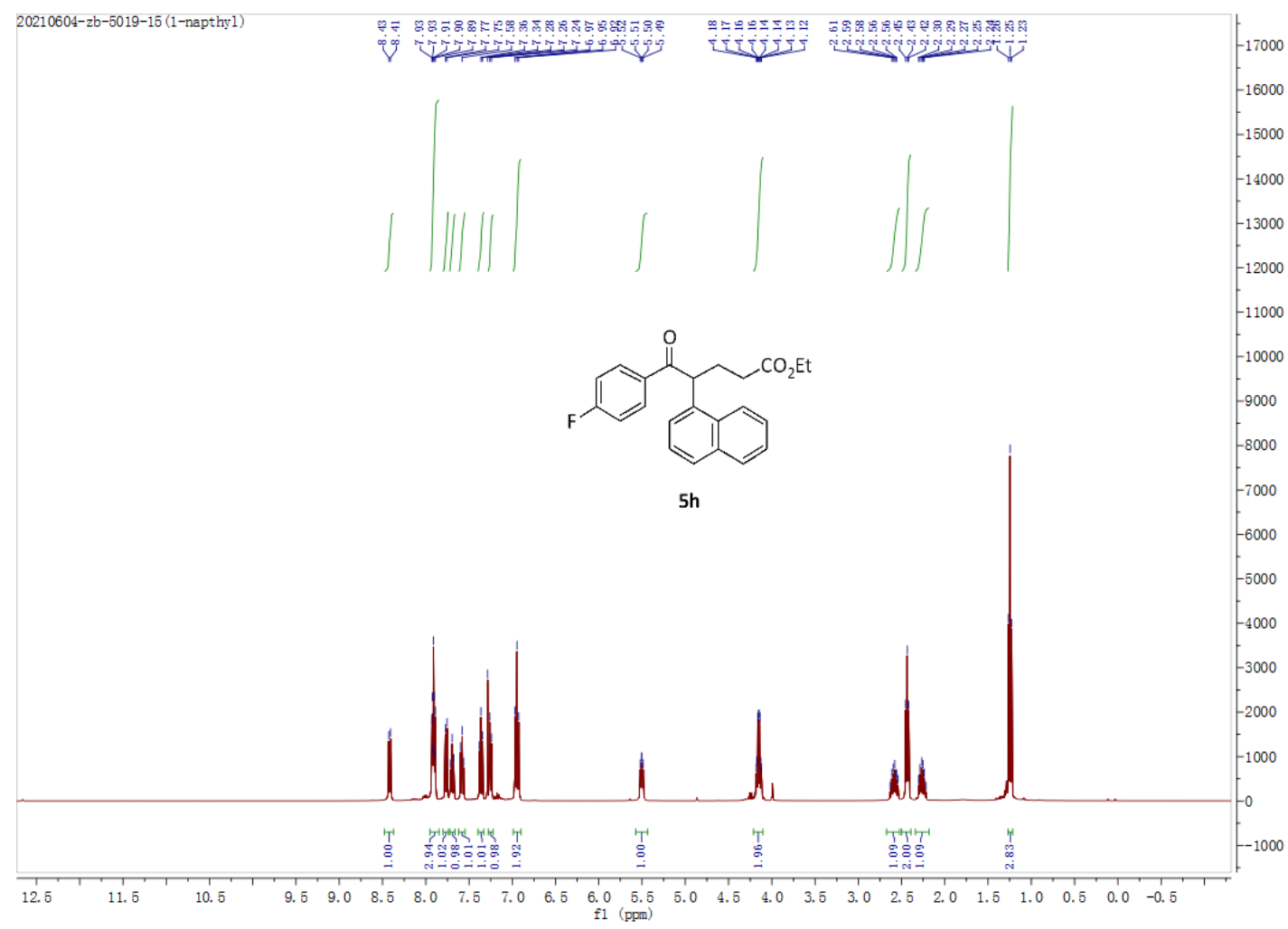

${ }^{19} \mathrm{~F}$ NMR $\left(\mathrm{CDCl}_{3}, 376 \mathrm{MHz}\right)$

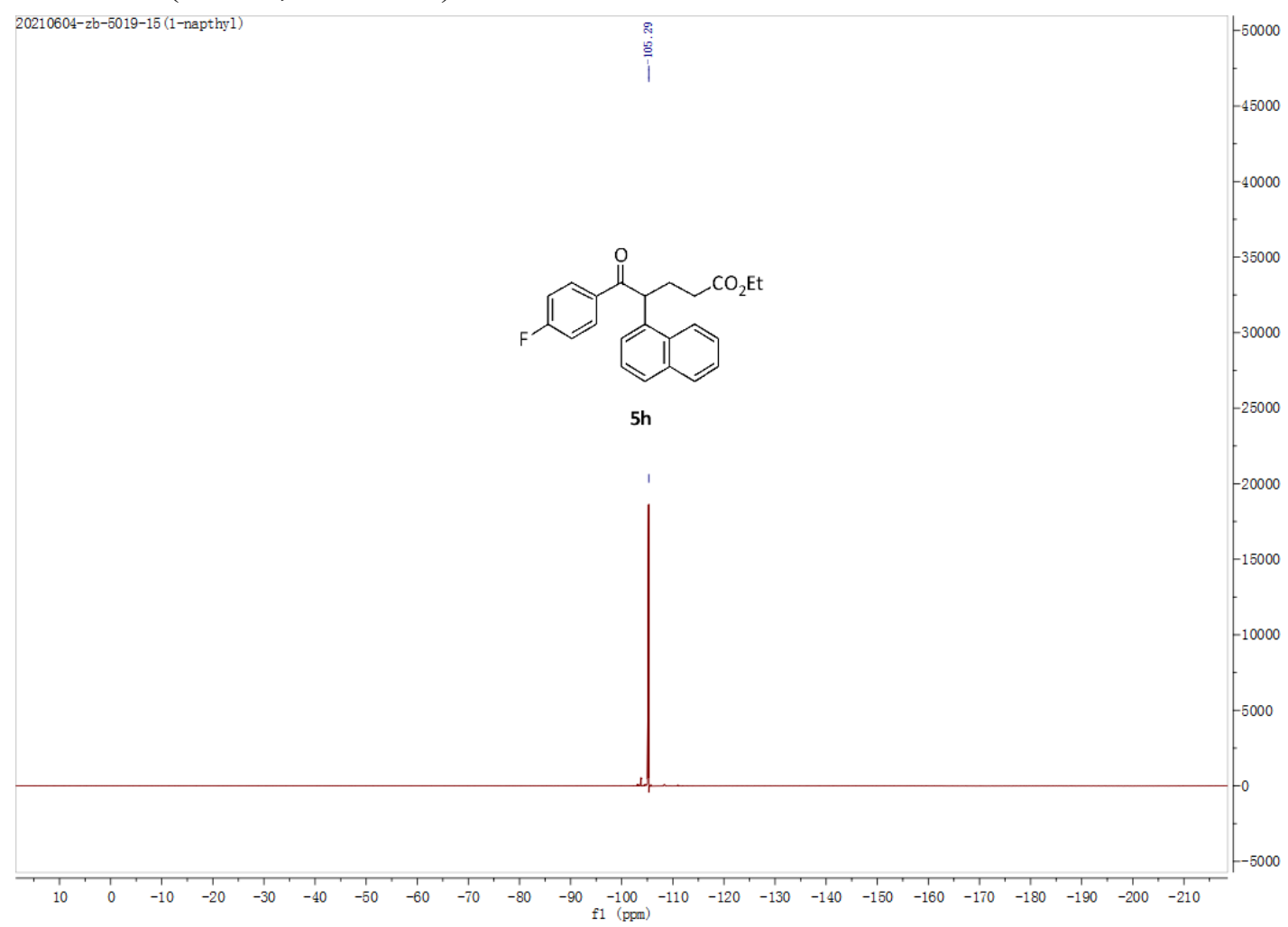


${ }^{13} \mathrm{C} \mathrm{NMR}\left(\mathrm{CDCl}_{3}, 100 \mathrm{MHz}\right)$

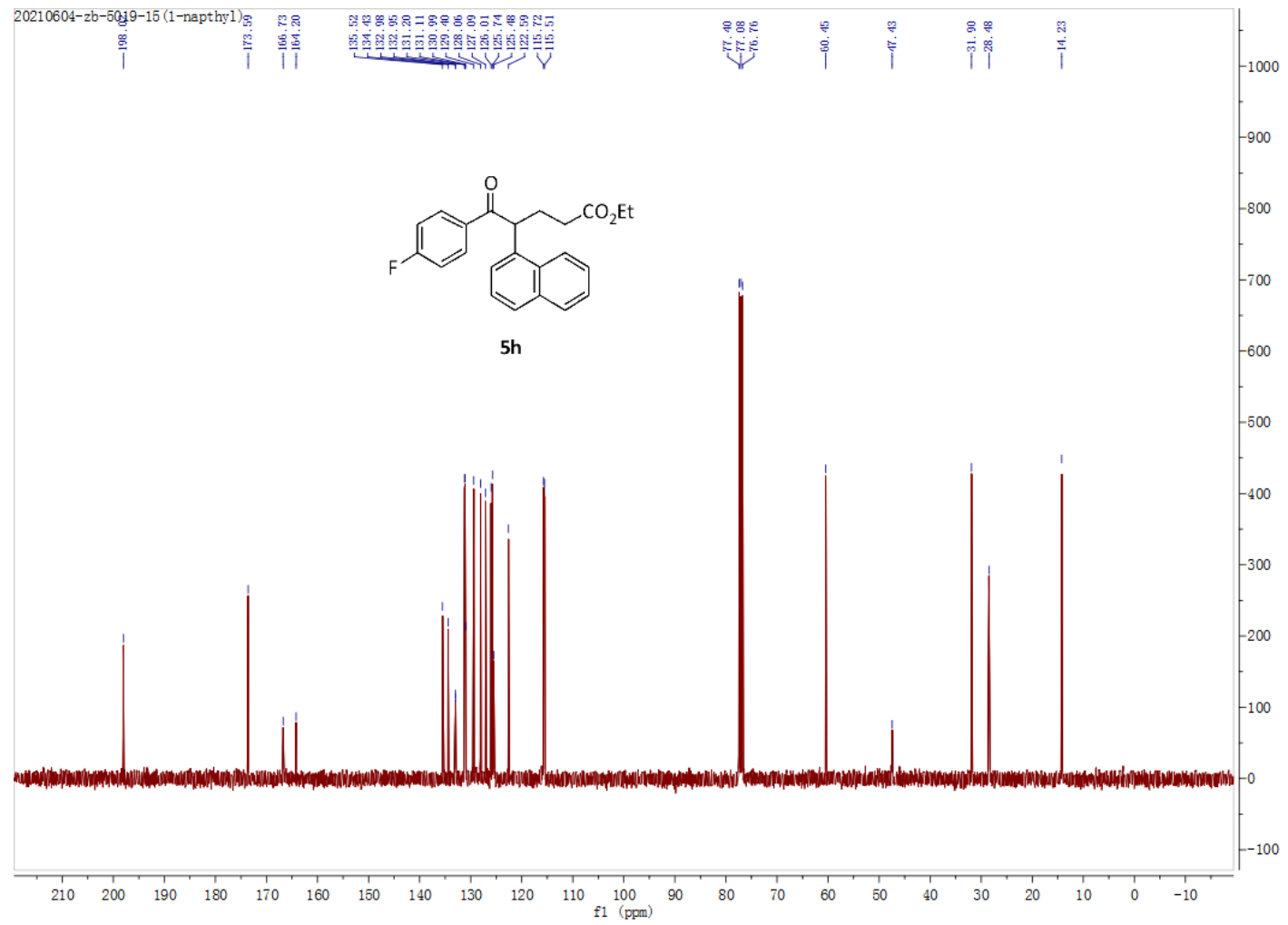




\section{Compound 5i}

${ }^{1} \mathrm{H} \mathrm{NMR}\left(\mathrm{CDCl}_{3}, 400 \mathrm{MHz}\right)$

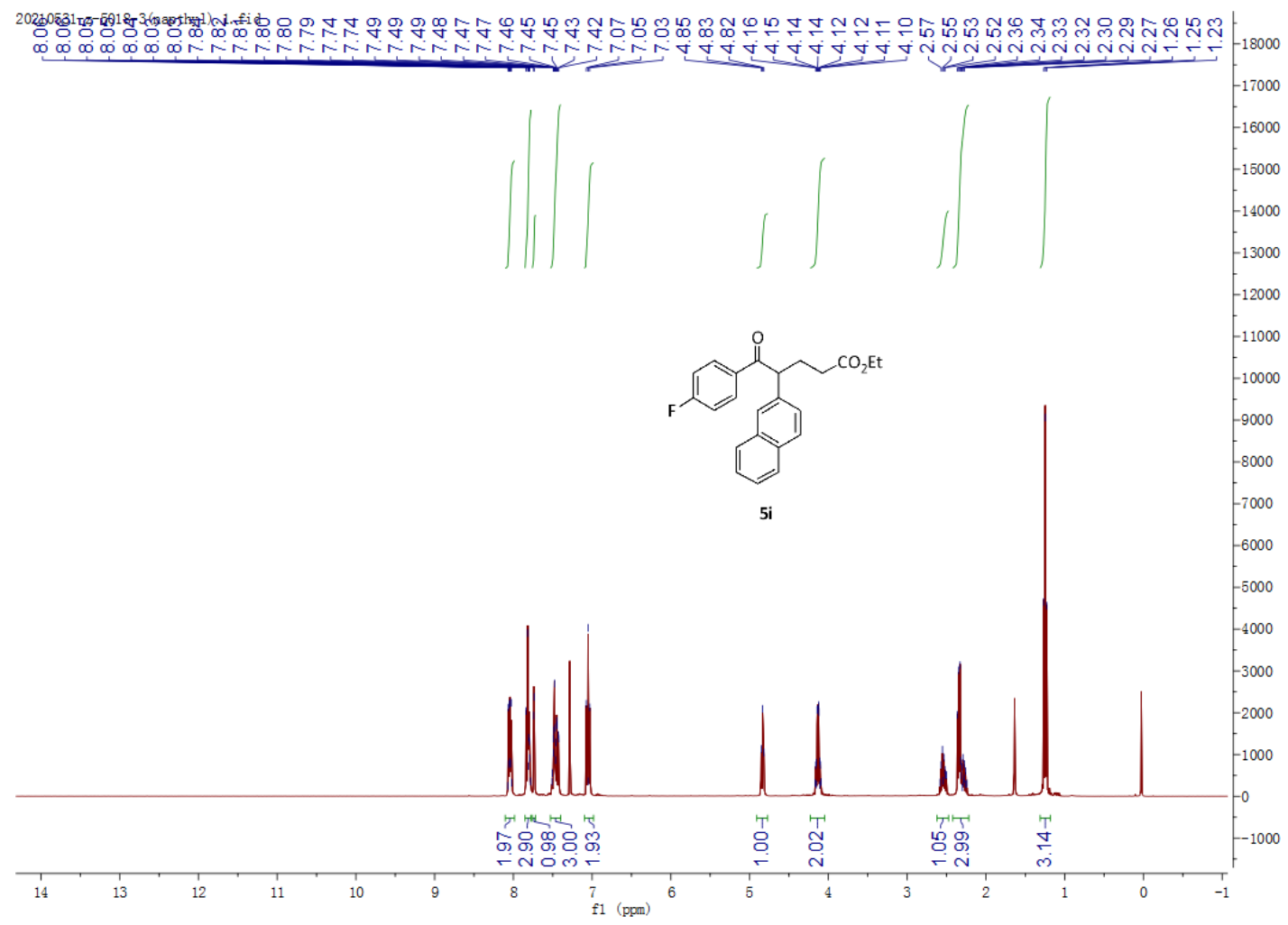

${ }^{19} \mathrm{~F} \mathrm{NMR}\left(\mathrm{CDCl}_{3}, 376 \mathrm{MHz}\right)$

20210531-z-5018-3 (nap thy 1) . 2. fid

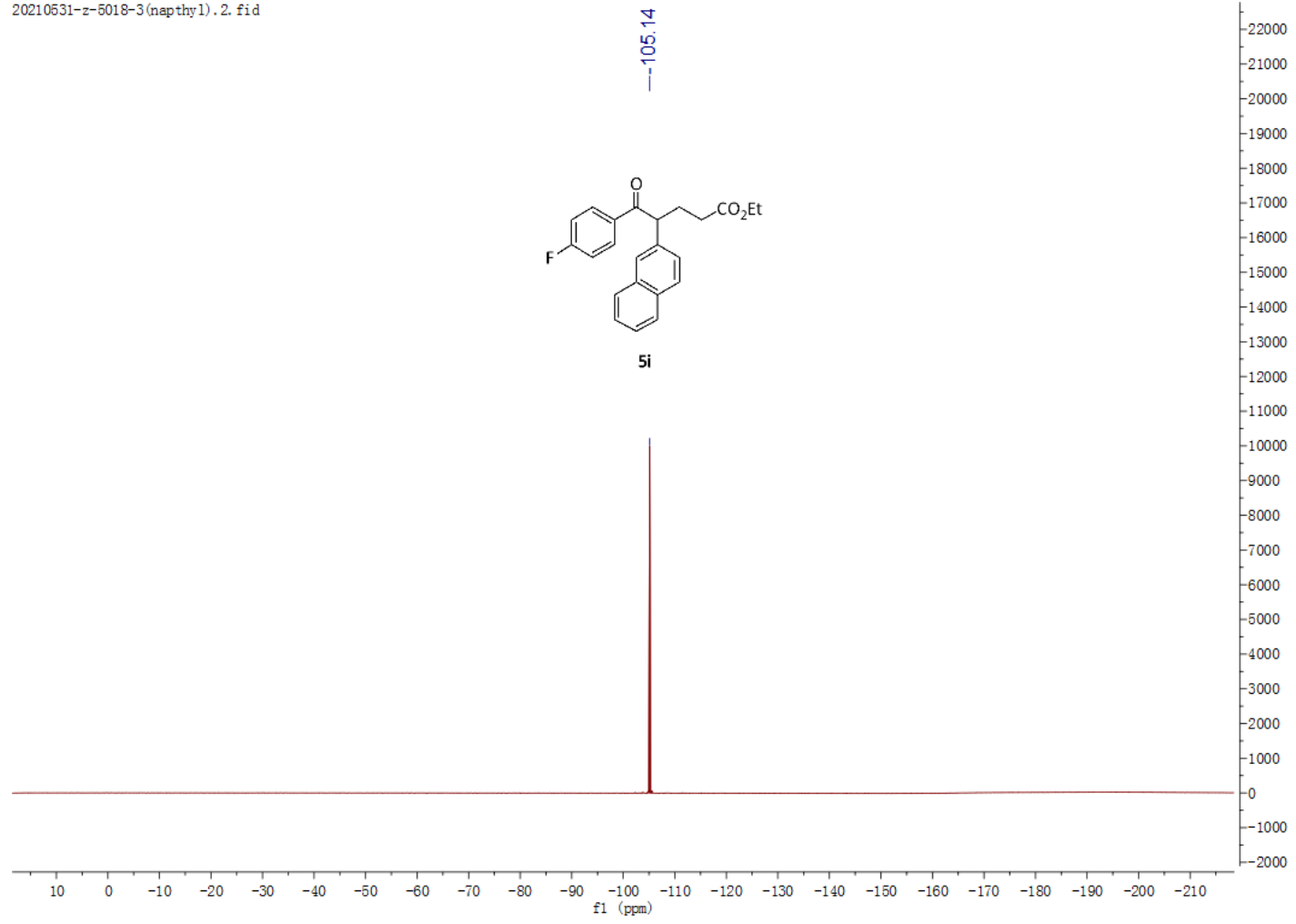


${ }^{13} \mathrm{C} \mathrm{NMR}\left(\mathrm{CDCl}_{3}, 100 \mathrm{MHz}\right)$

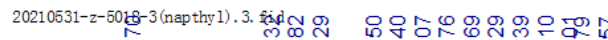

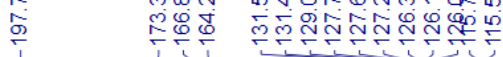

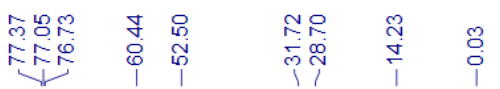

$\int_{-4500}^{-5000}$

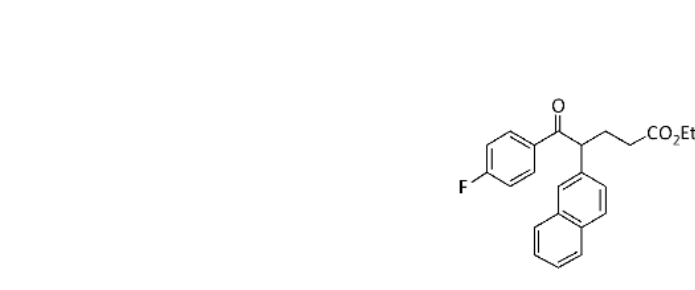

$5 i$

4000

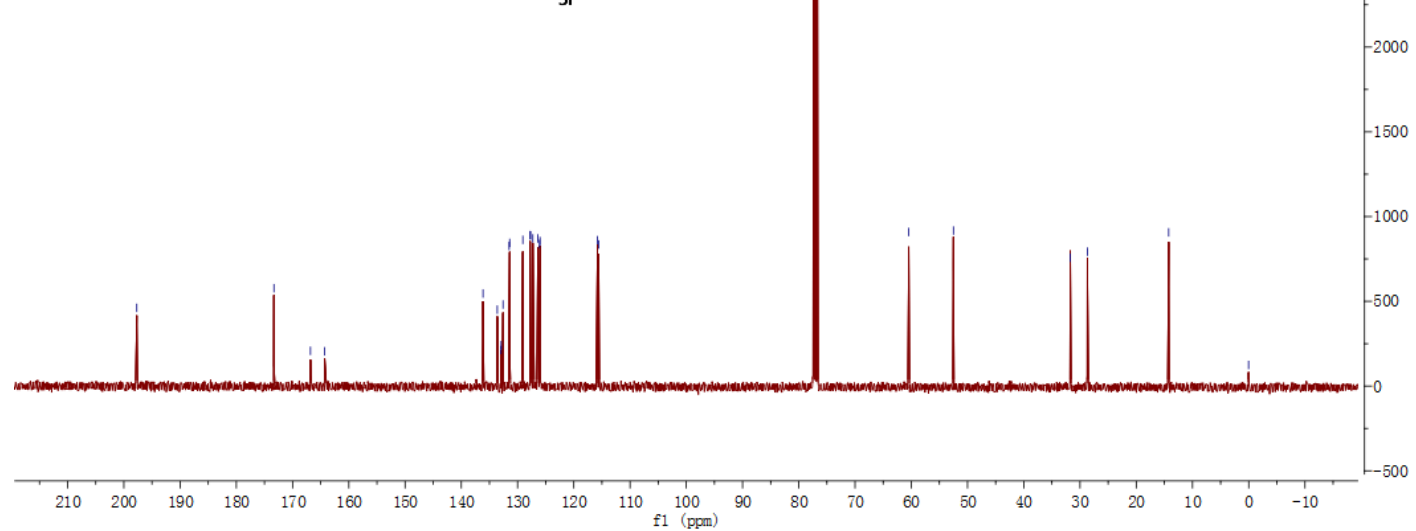




\section{Compound $\mathbf{5 j}$}

${ }^{1} \mathrm{H} \mathrm{NMR}\left(\mathrm{CDCl}_{3}, 400 \mathrm{MHz}\right)$

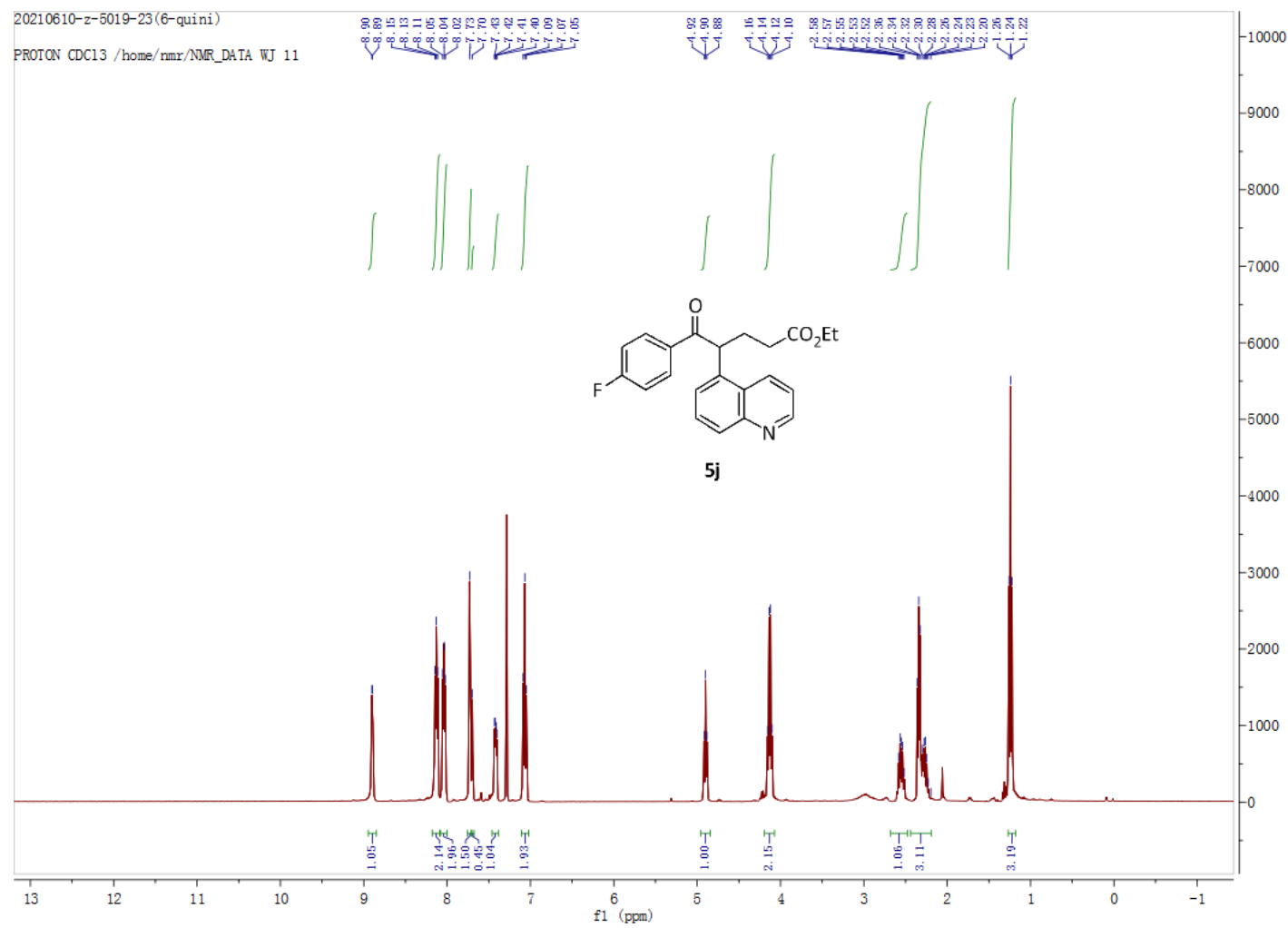

${ }^{19} \mathrm{~F}$ NMR $\left(\mathrm{CDCl}_{3}, 376 \mathrm{MHz}\right)$

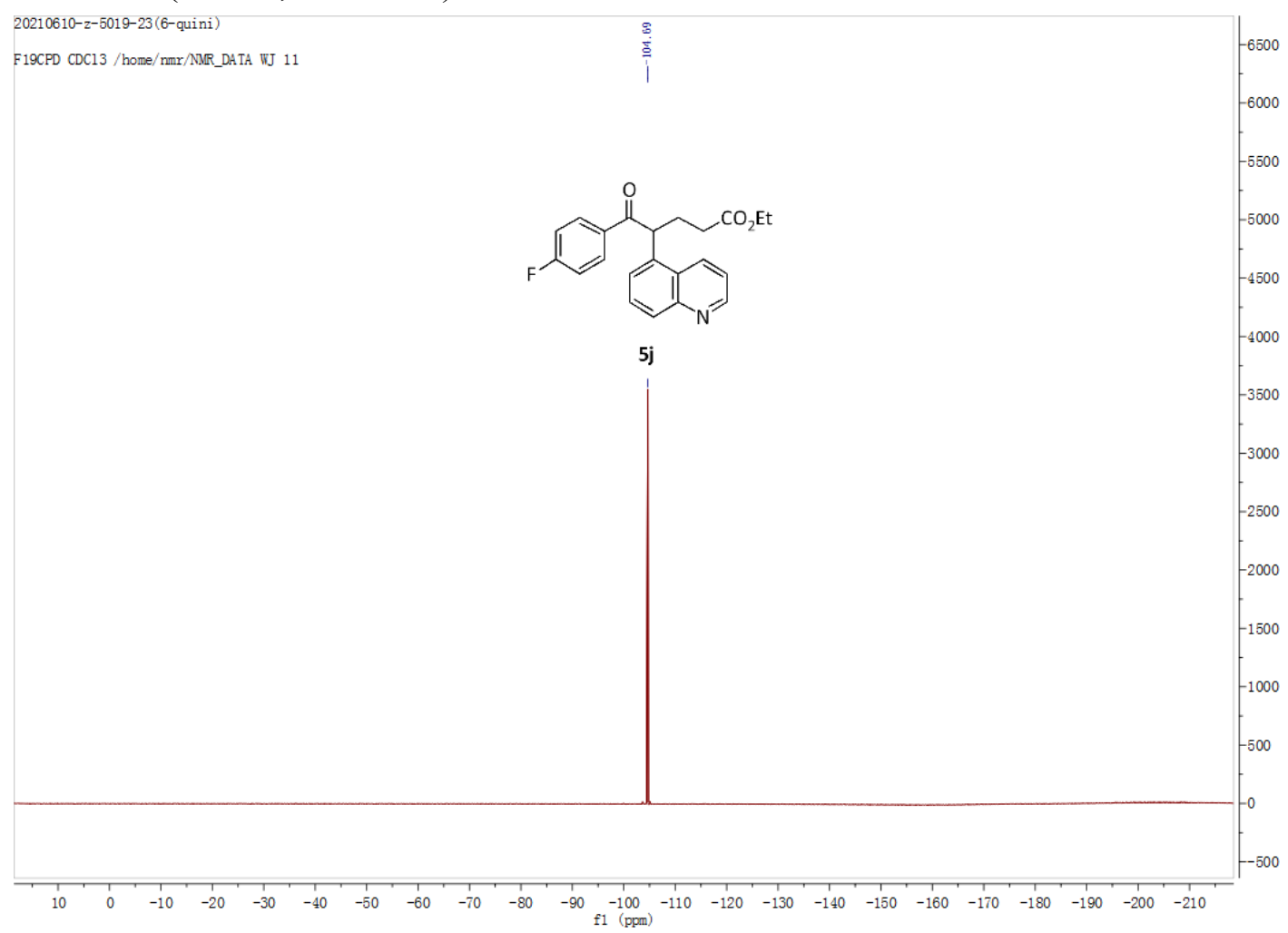


${ }^{13} \mathrm{C} \mathrm{NMR}\left(\mathrm{CDCl}_{3}, 100 \mathrm{MHz}\right)$

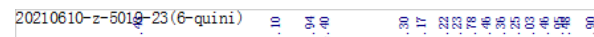

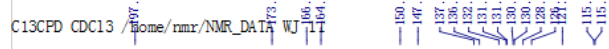

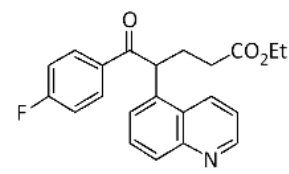

$5 j$
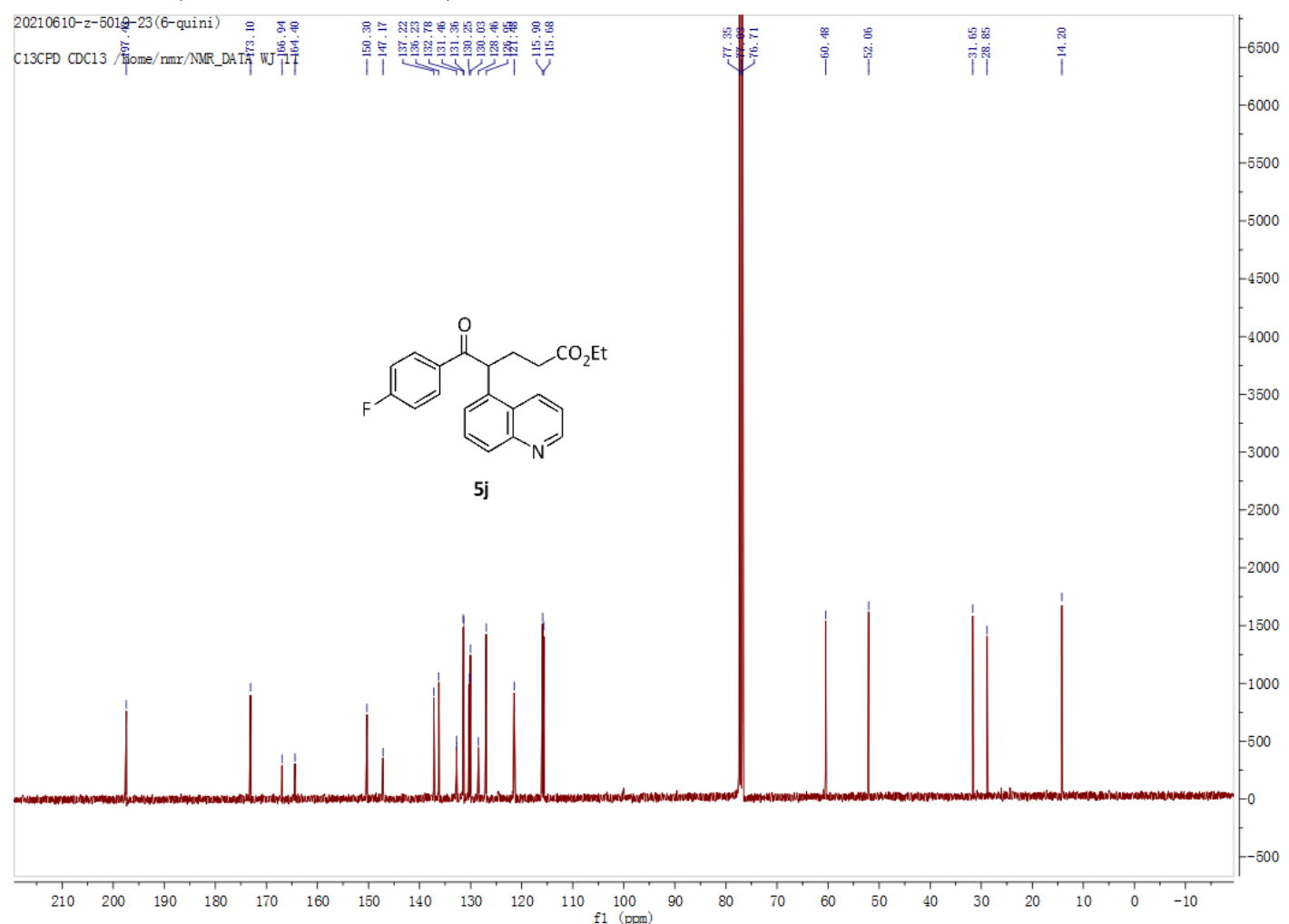


\section{Compound 5k}

${ }^{1} \mathrm{H}$ NMR (MeOD, $400 \mathrm{MHz}$ )

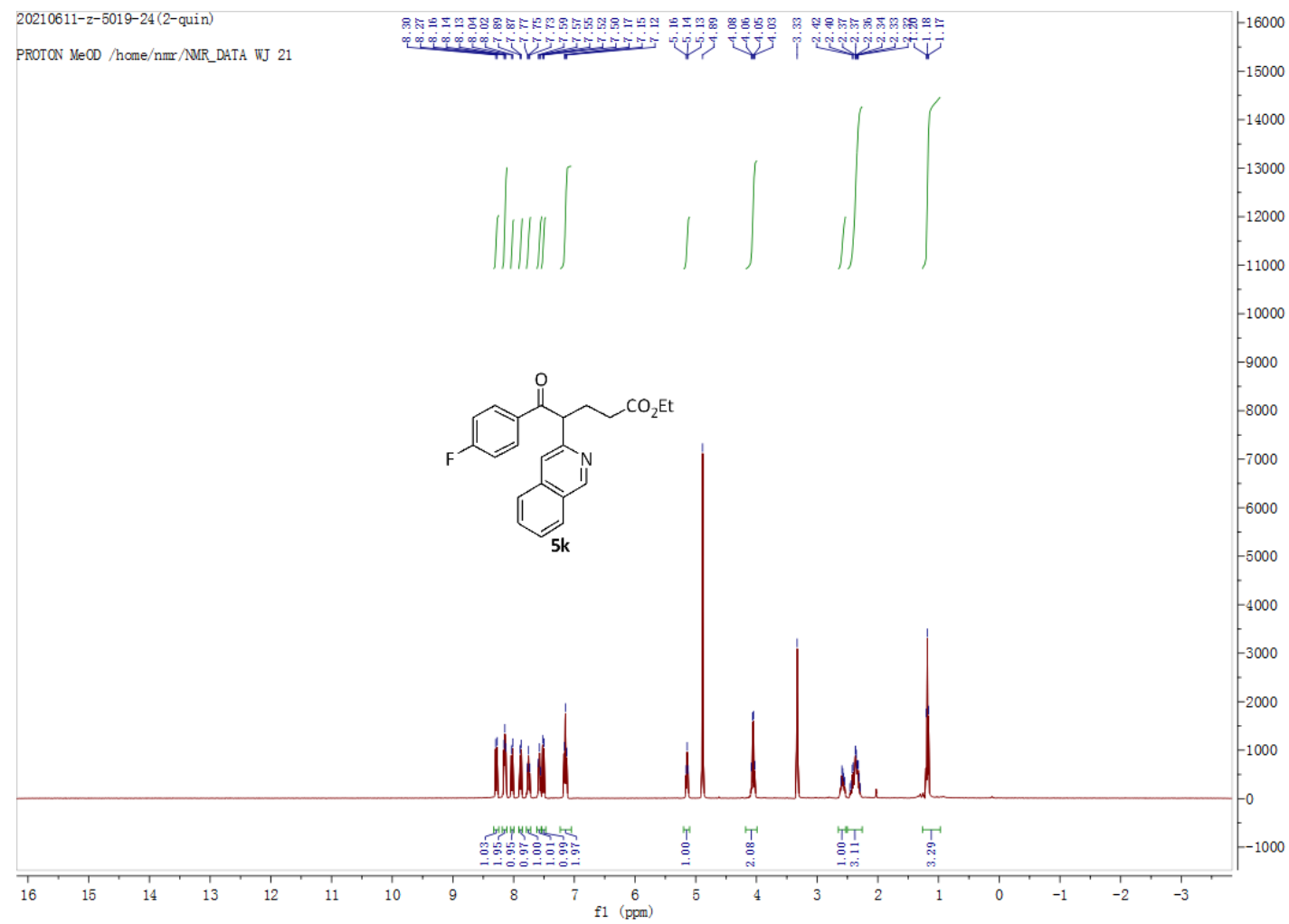

${ }^{19}$ F NMR (MeOD, $376 \mathrm{MHz}$ )

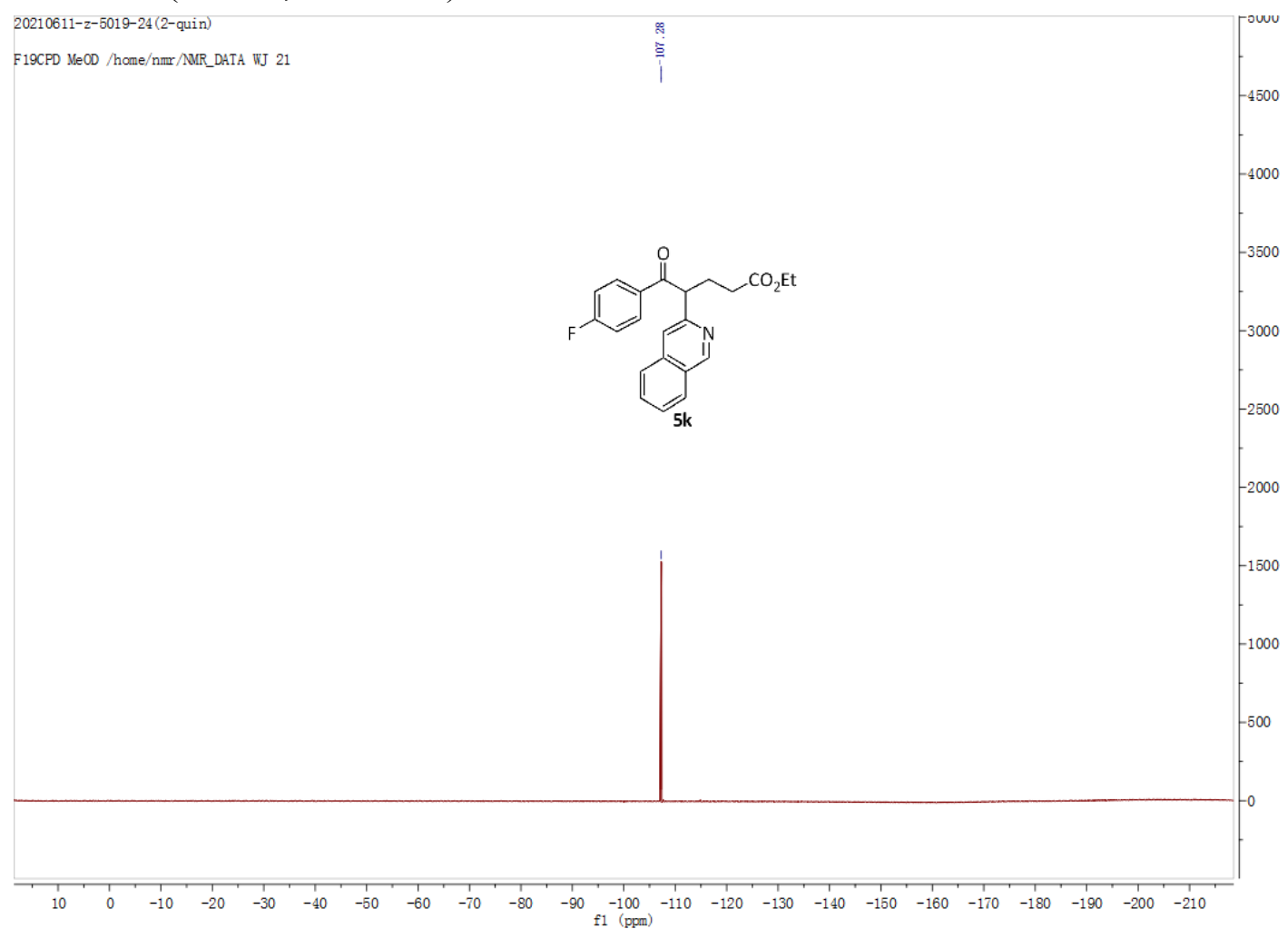


${ }^{13} \mathrm{C}$ NMR (MeOD, $100 \mathrm{MHz}$ )

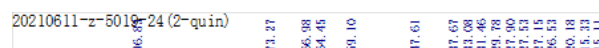

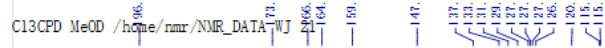
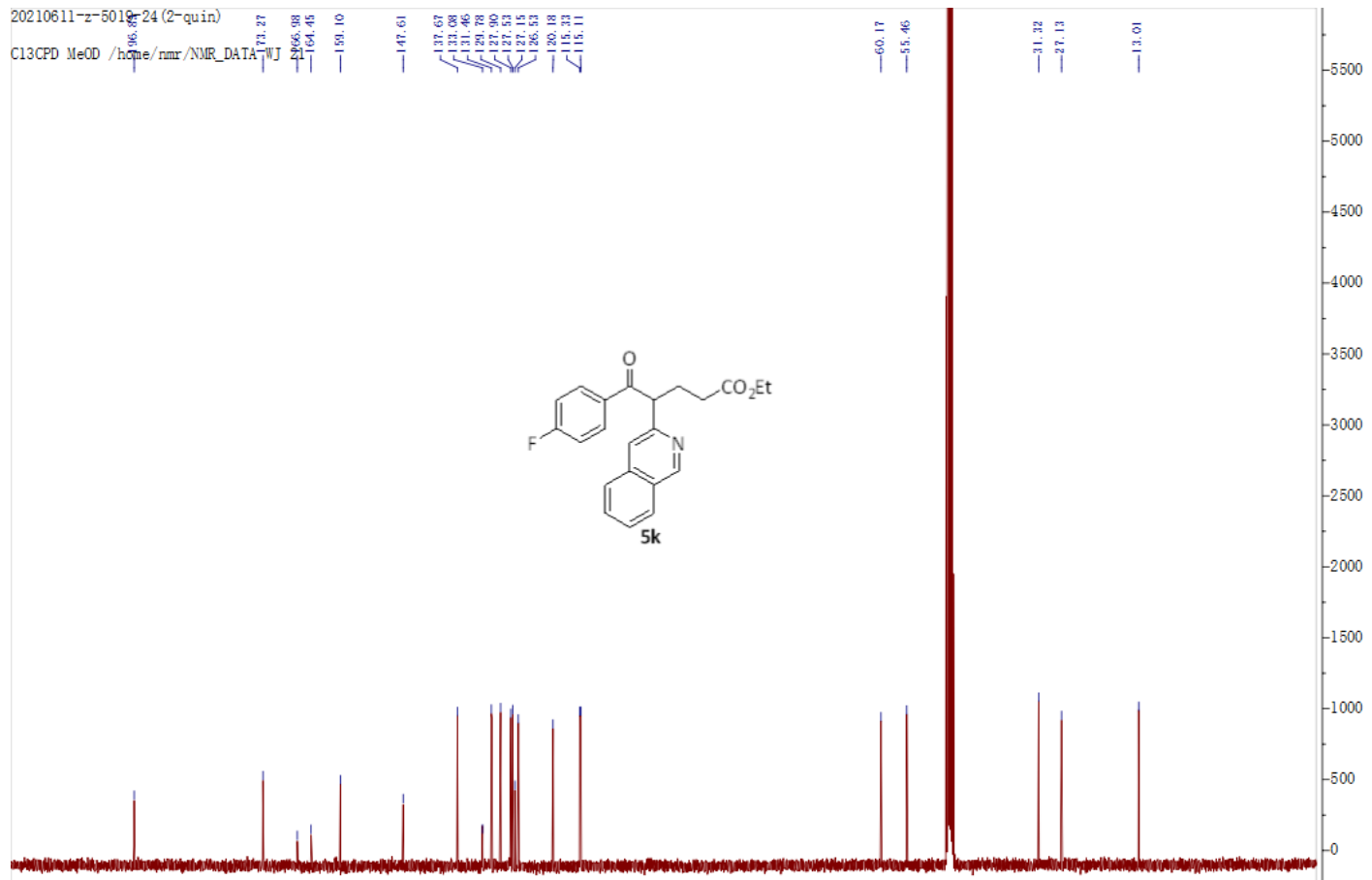

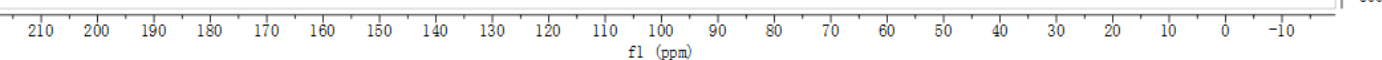




\section{Compound $5 \mathbf{I}$}

${ }^{1} \mathrm{H} \mathrm{NMR}\left(\mathrm{CDCl}_{3}, 400 \mathrm{MHz}\right)$

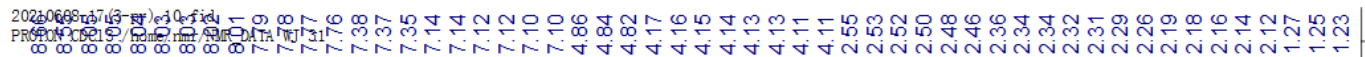

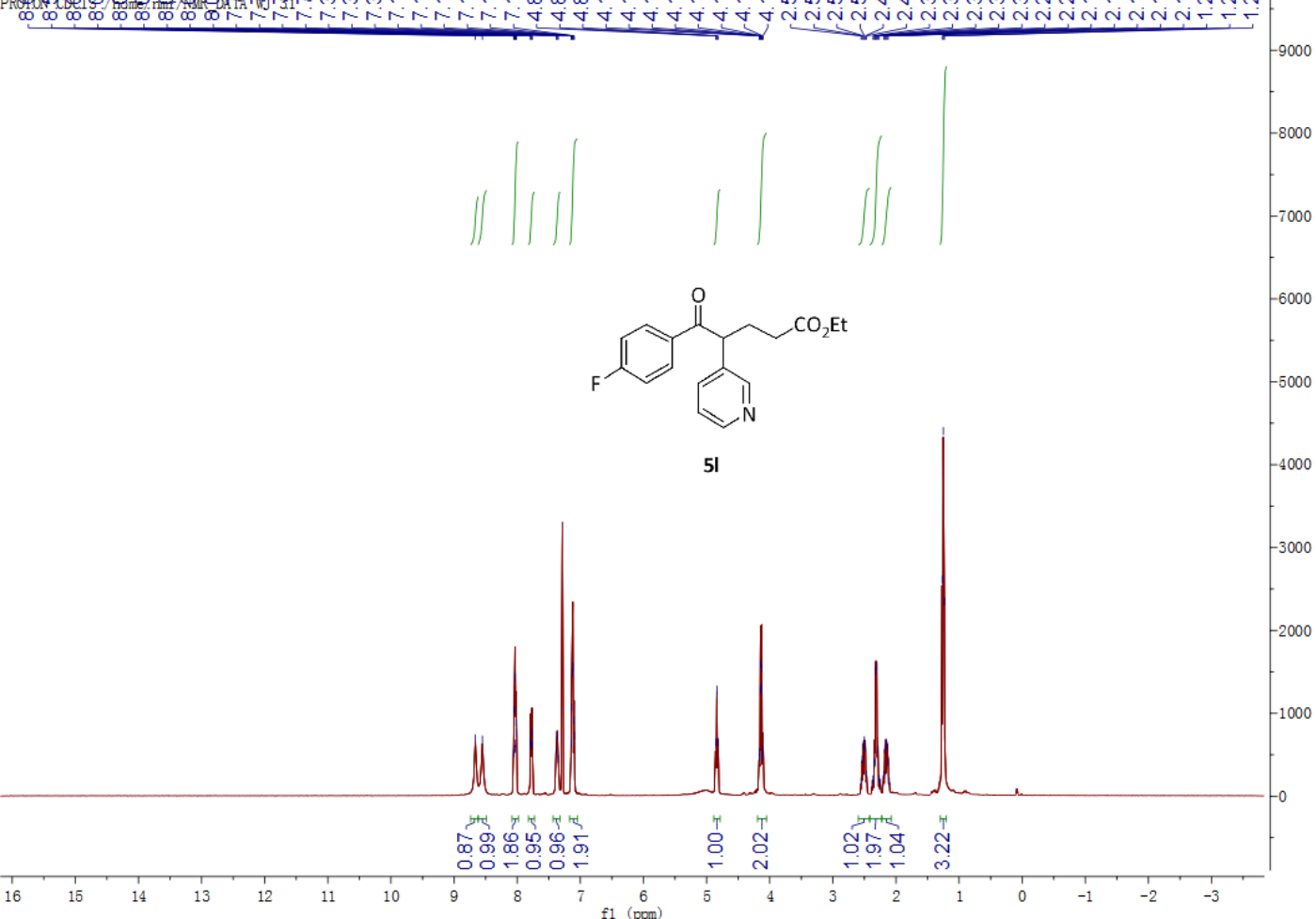

${ }^{19} \mathrm{~F} \mathrm{NMR}\left(\mathrm{CDCl}_{3}, 376 \mathrm{MHz}\right)$

20210608-17 (3-py).11. fid

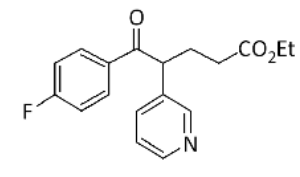


${ }^{13} \mathrm{C} \mathrm{NMR}\left(\mathrm{CDCl}_{3}, 100 \mathrm{MHz}\right)$

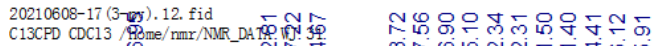

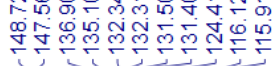

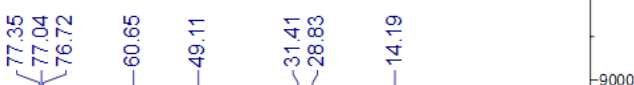

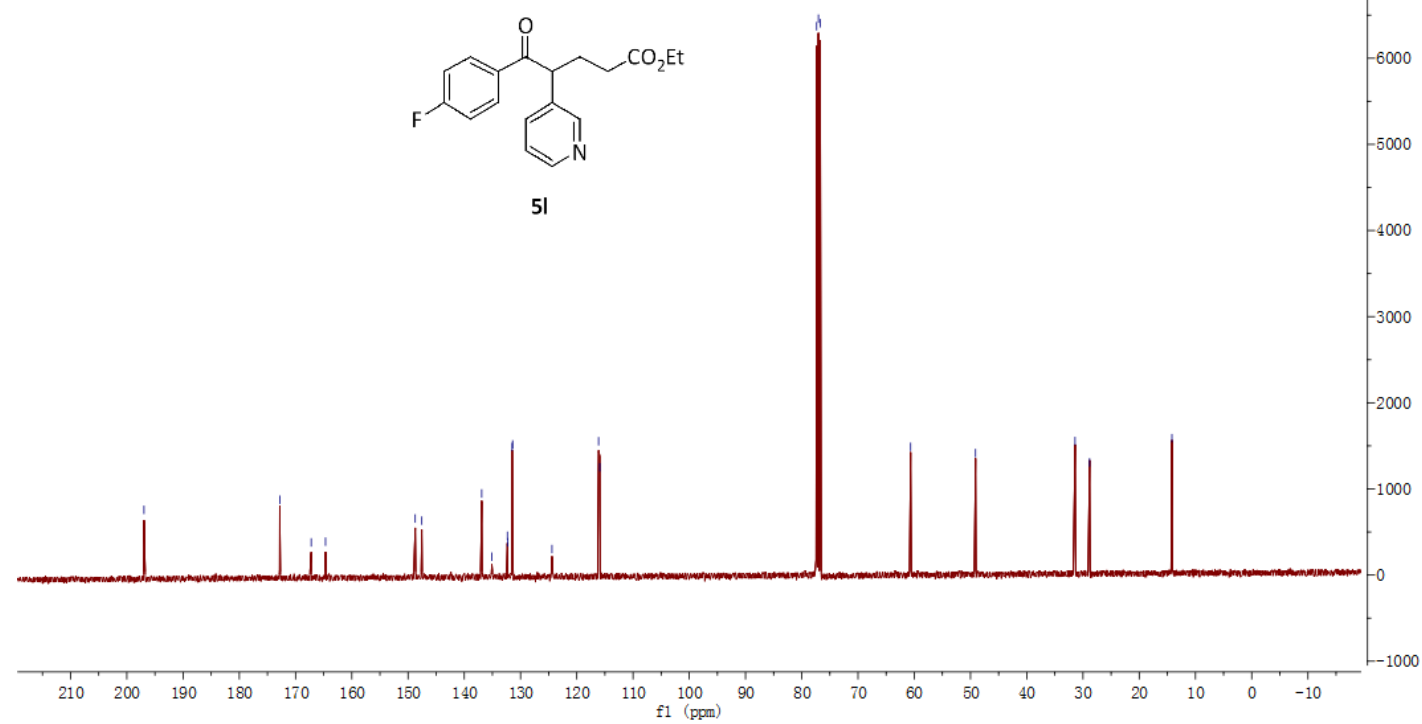

51

8000

7000 


\section{Compound 5m}

${ }^{1} \mathrm{H} \mathrm{NMR}\left(\mathrm{CDCl}_{3}, 400 \mathrm{MHz}\right)$

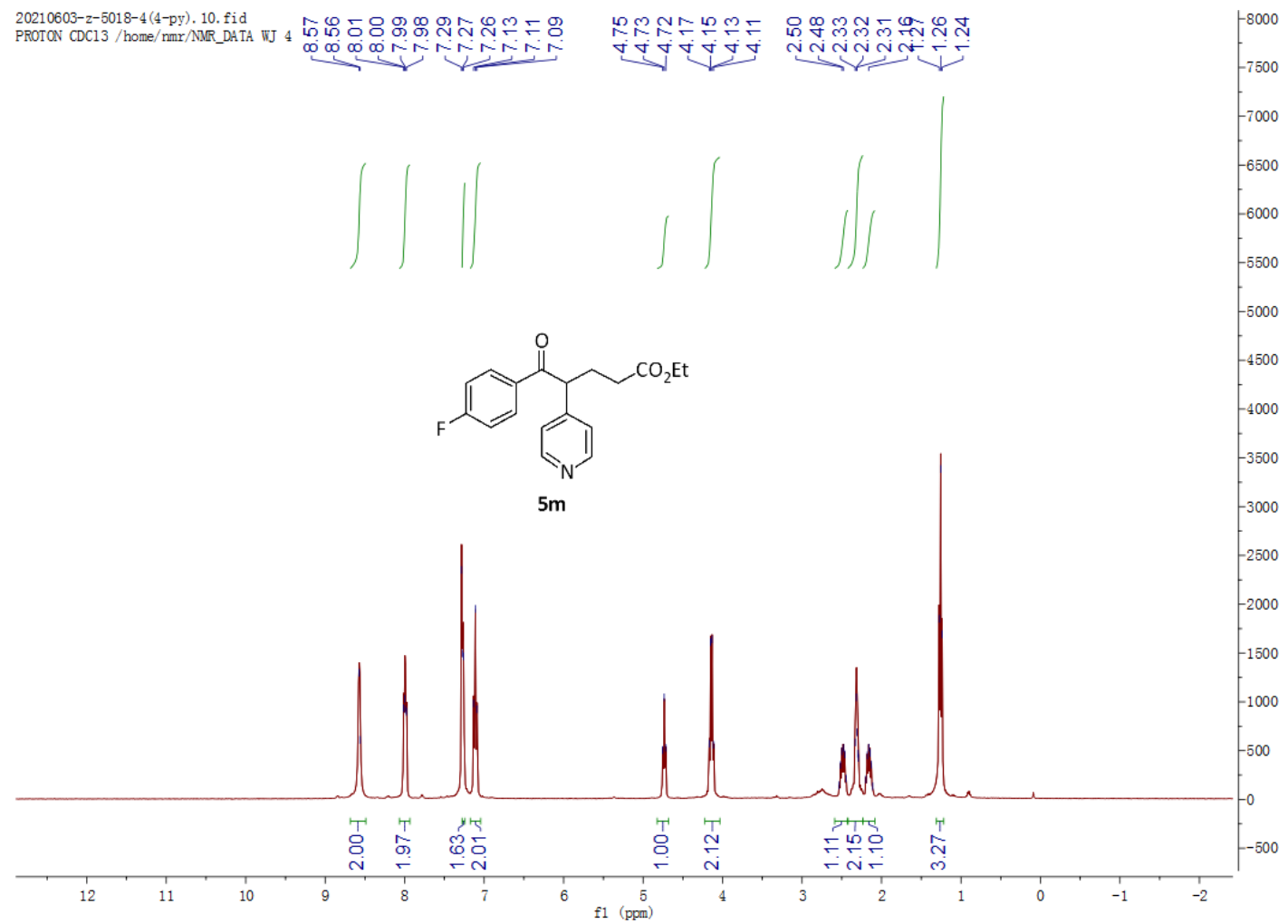

${ }^{19} \mathrm{~F} \mathrm{NMR}\left(\mathrm{CDCl}_{3}, 376 \mathrm{MHz}\right)$

20210603-z-5018-4 (4-py). 11. fid
F19CPD CDC13 /home/nmr/NMR_DATA WJ 4

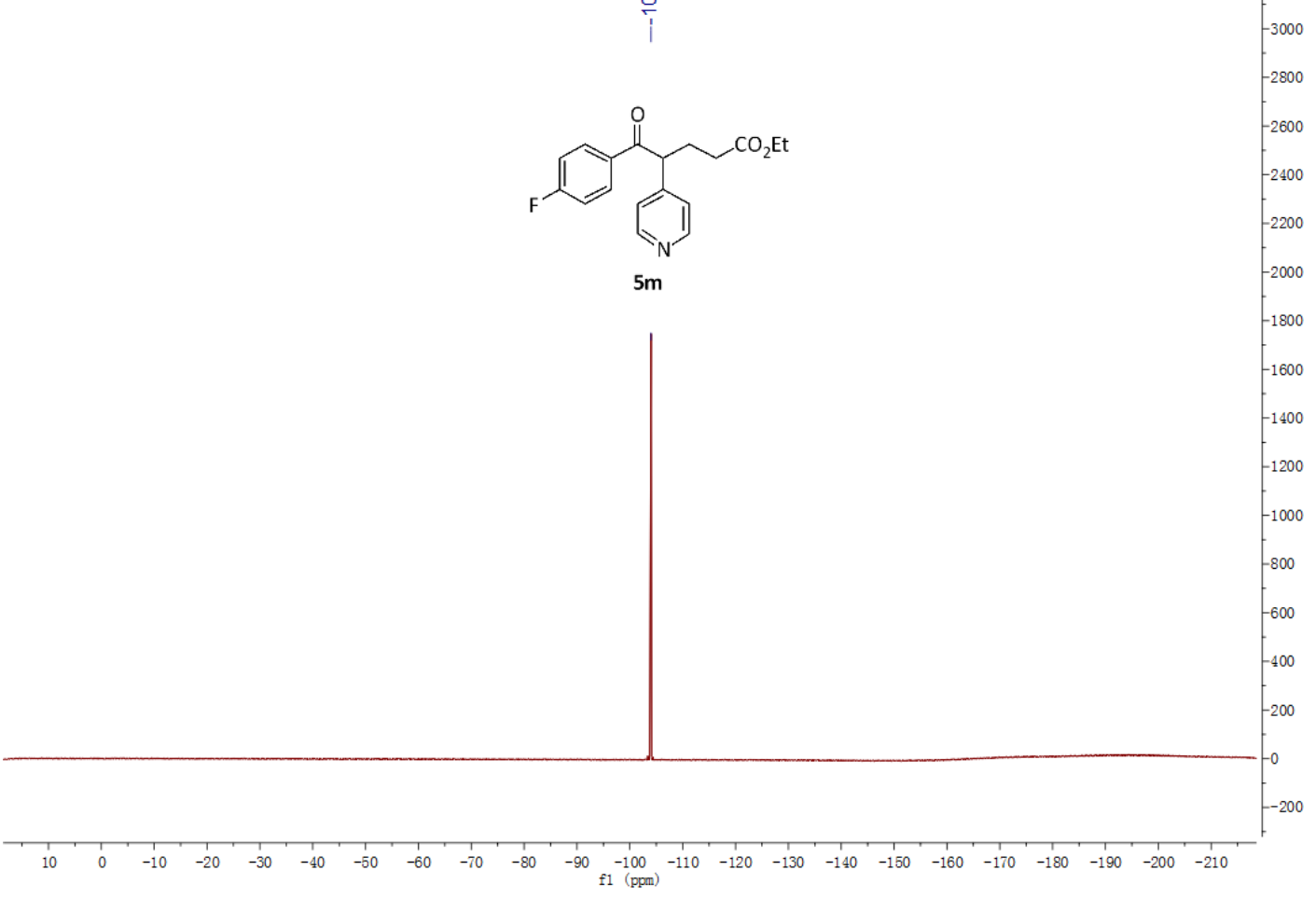


${ }^{13} \mathrm{C} \mathrm{NMR}\left(\mathrm{CDCl}_{3}, 100 \mathrm{MHz}\right)$

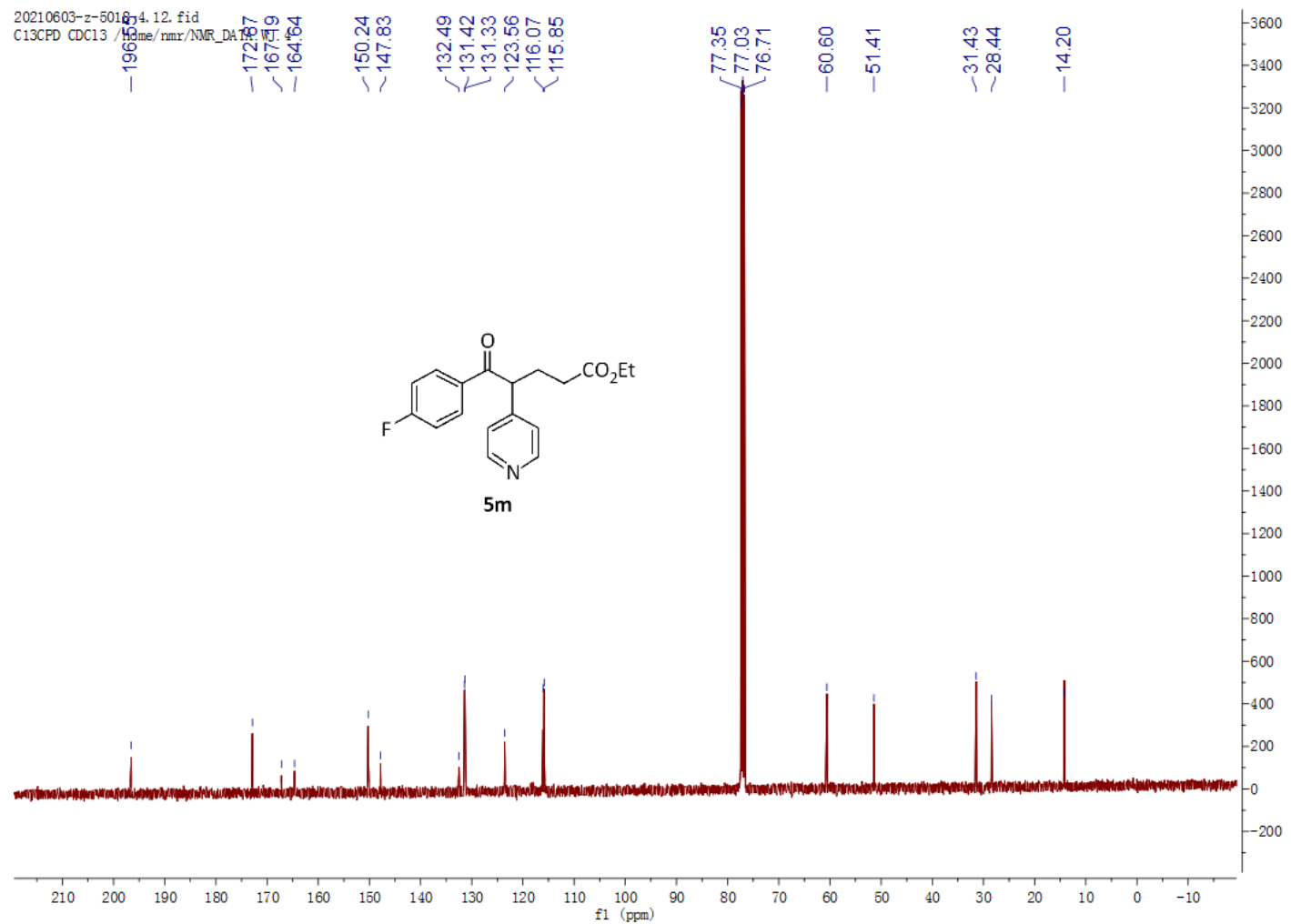




\section{Compound 5n}

${ }^{1} \mathrm{H} \mathrm{NMR}\left(\mathrm{CDCl}_{3}, 400 \mathrm{MHz}\right)$

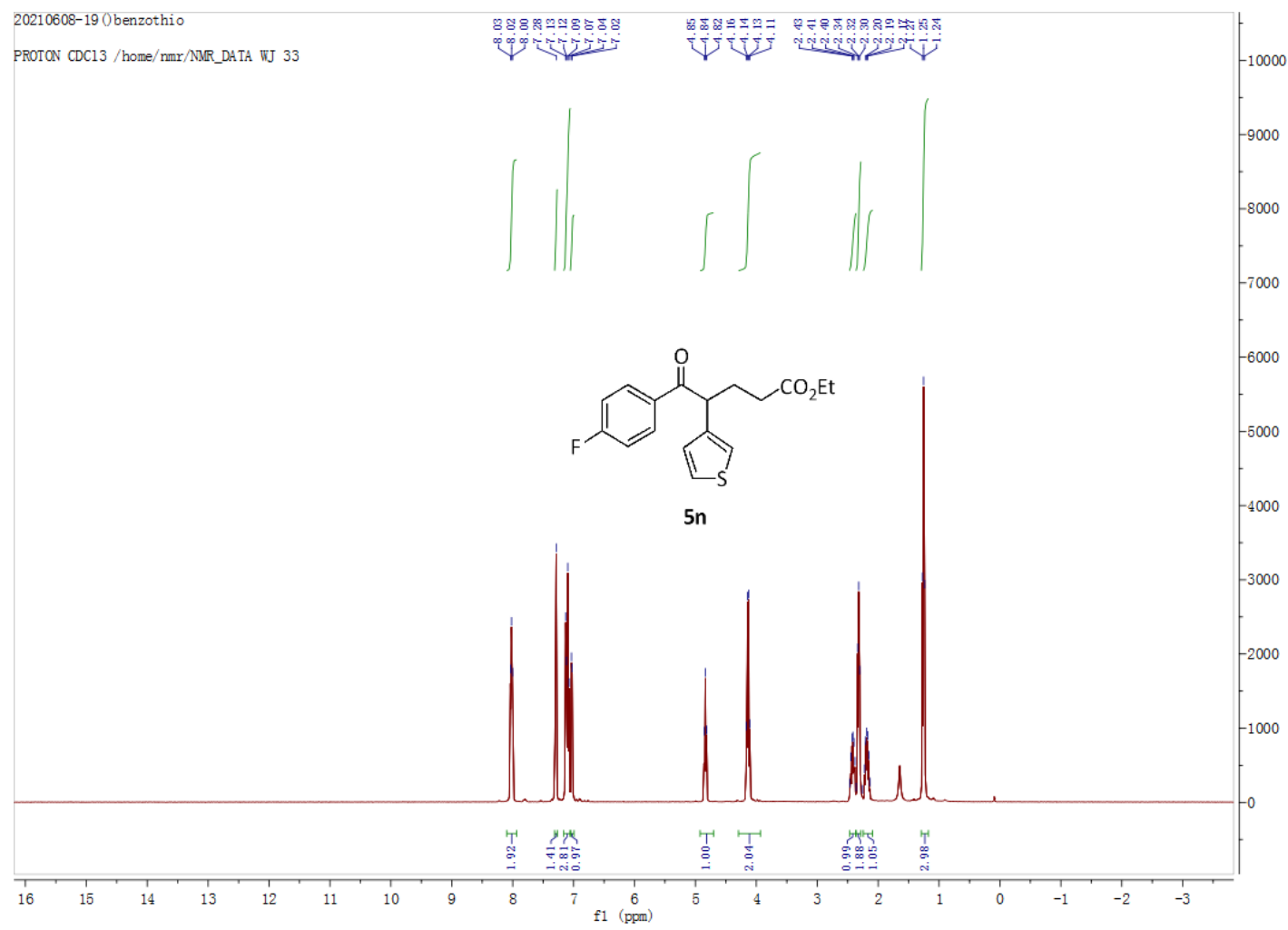

${ }^{13} \mathrm{C} \mathrm{NMR}\left(\mathrm{CDCl}_{3}, 100 \mathrm{MHz}\right)$

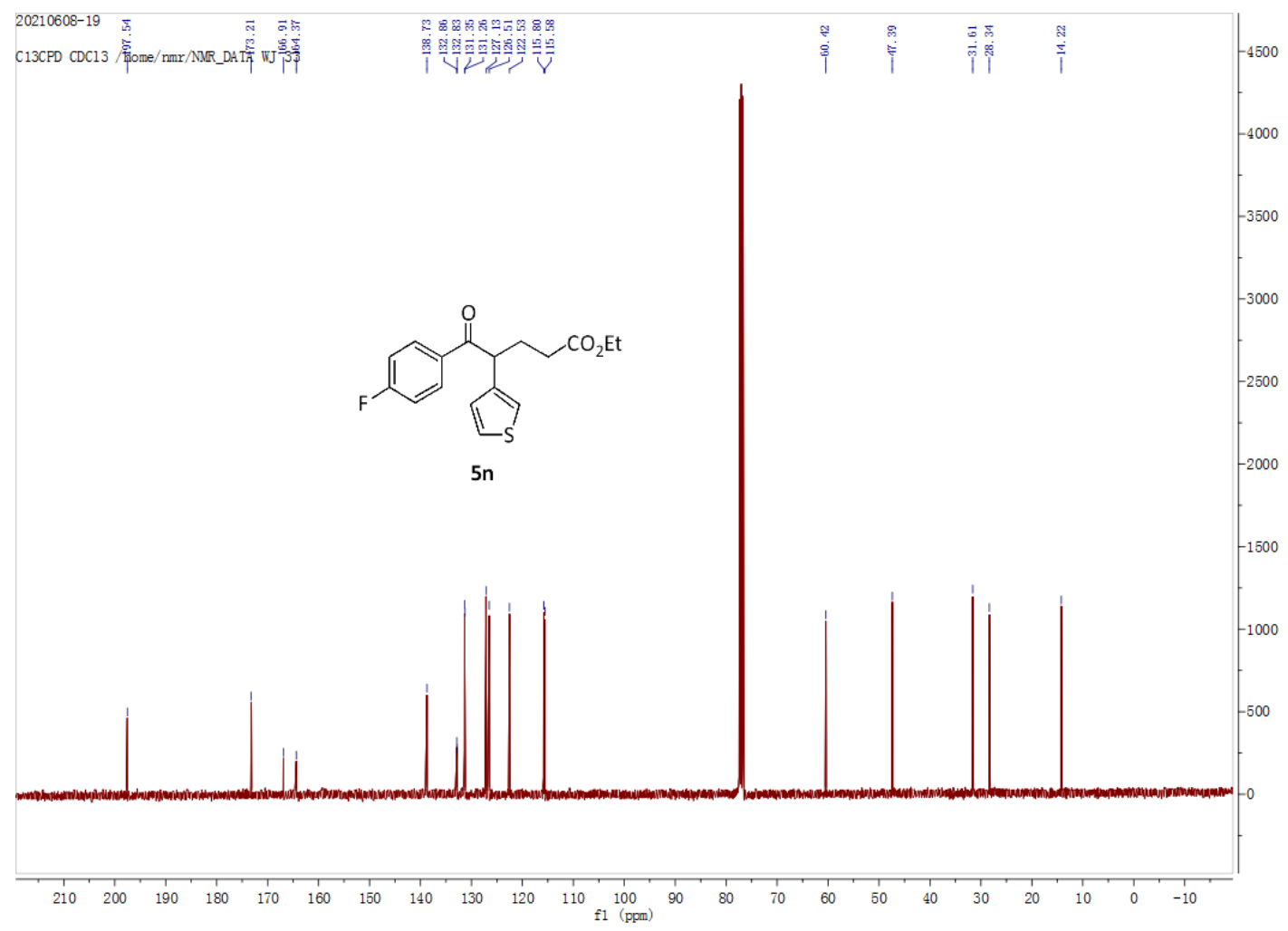


${ }^{19} \mathrm{~F} \mathrm{NMR}\left(\mathrm{CDCl}_{3}, 376 \mathrm{MHz}\right)$

20210608-19 () benzothio

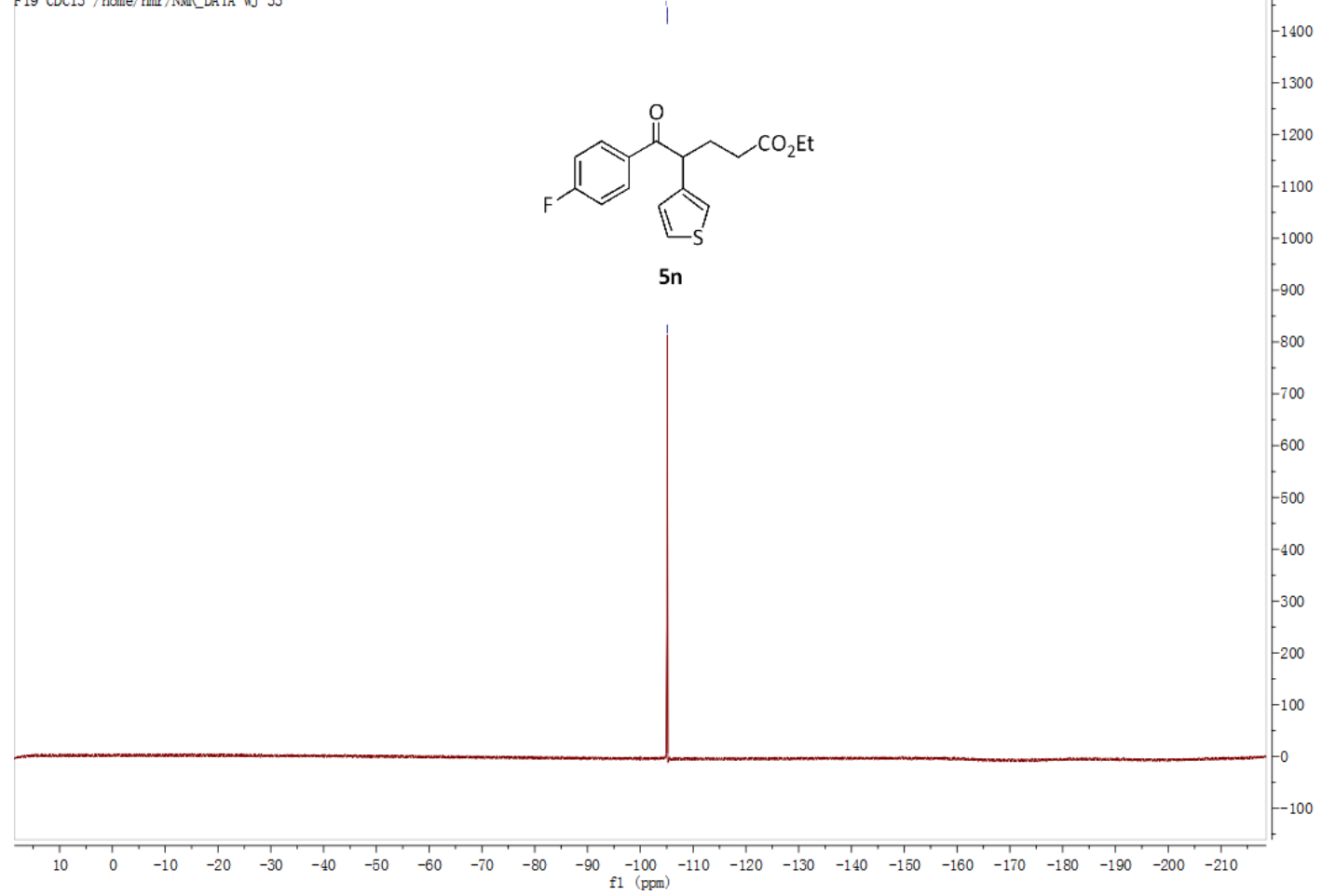




\section{Compound 50}

${ }^{1} \mathrm{H} \mathrm{NMR}\left(\mathrm{CDCl}_{3}, 400 \mathrm{MHz}\right)$

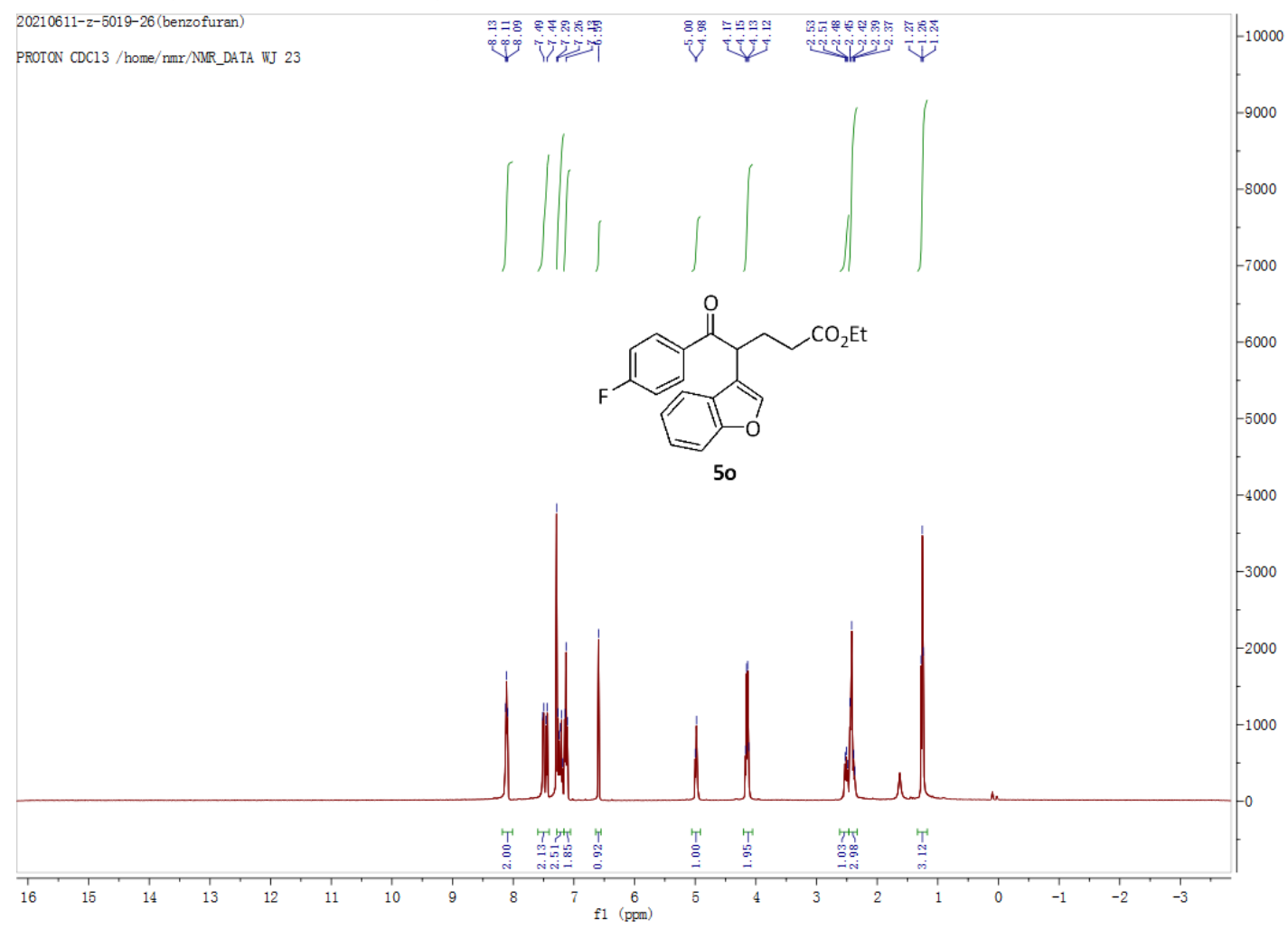

${ }^{19} \mathrm{~F}$ NMR $\left(\mathrm{CDCl}_{3}, 376 \mathrm{MHz}\right)$

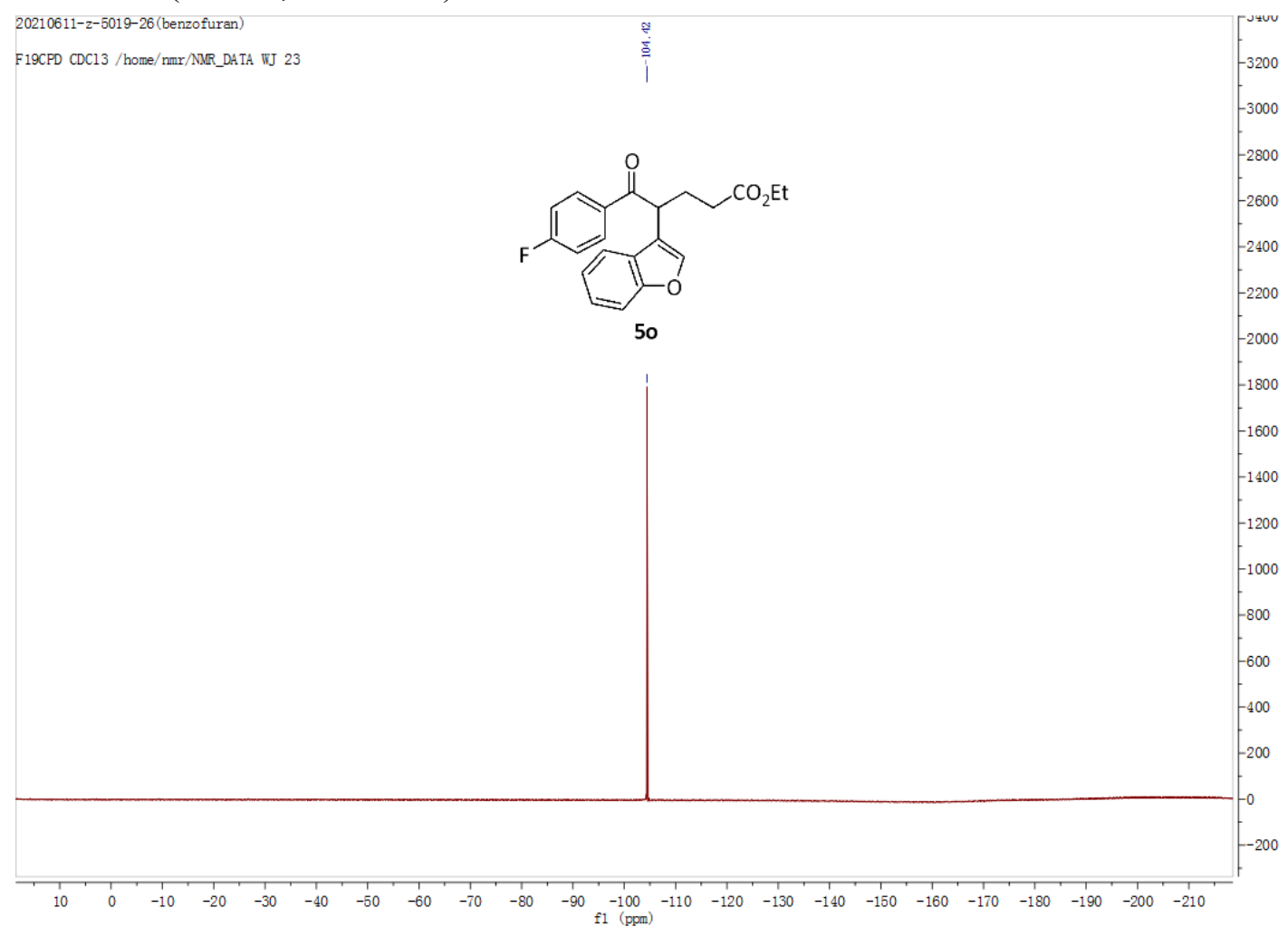


${ }^{13} \mathrm{C} \mathrm{NMR}\left(\mathrm{CDCl}_{3}, 100 \mathrm{MHz}\right)$

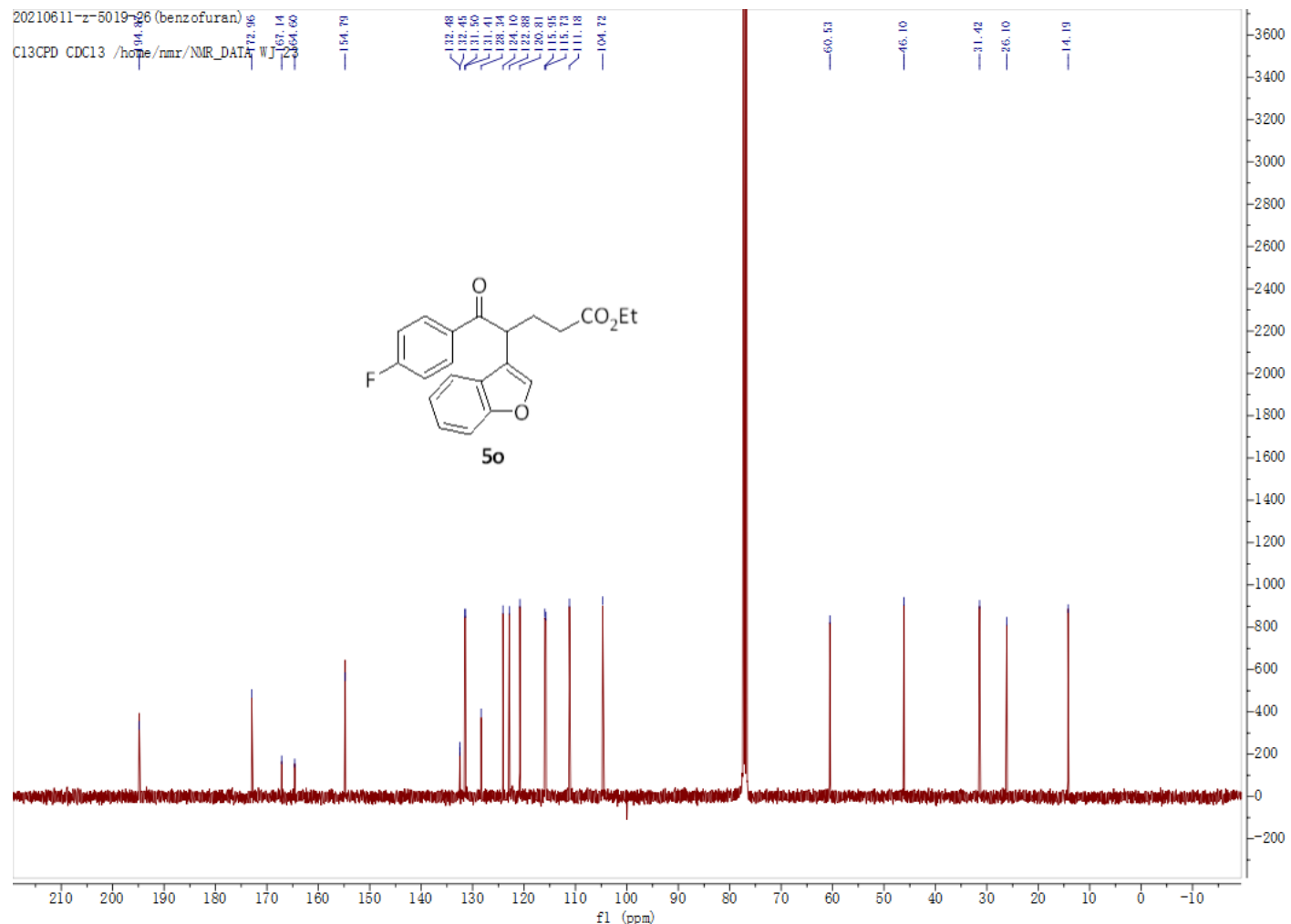




\section{Compound 5p}

${ }^{1} \mathrm{H} \mathrm{NMR}\left(\mathrm{CDCl}_{3}, 400 \mathrm{MHz}\right)$

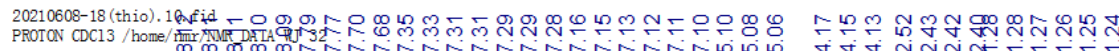

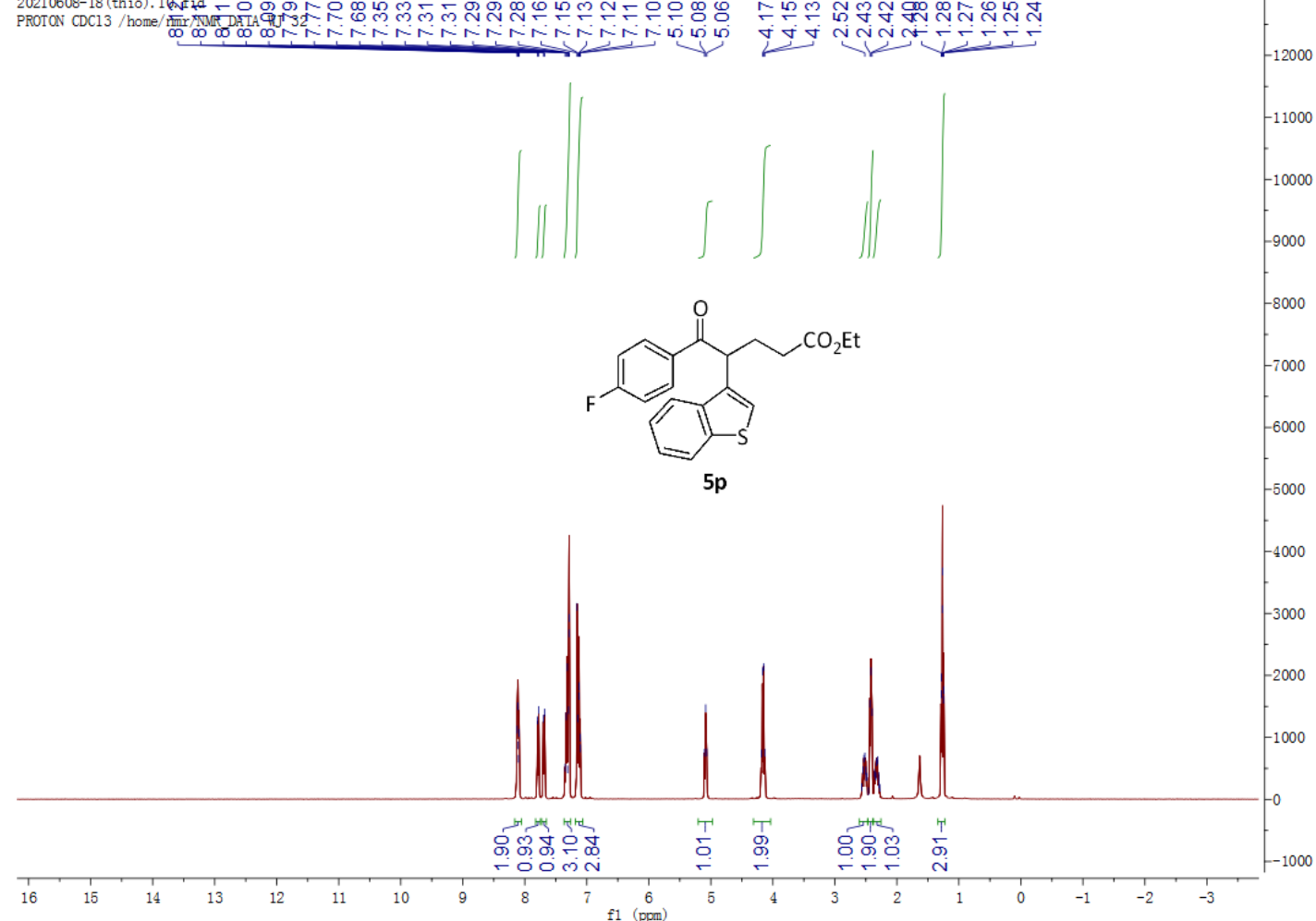

${ }^{19} \mathrm{~F}$ NMR $\left(\mathrm{CDCl}_{3}, 376 \mathrm{MHz}\right)$

20210608-18 (thio).11. fid
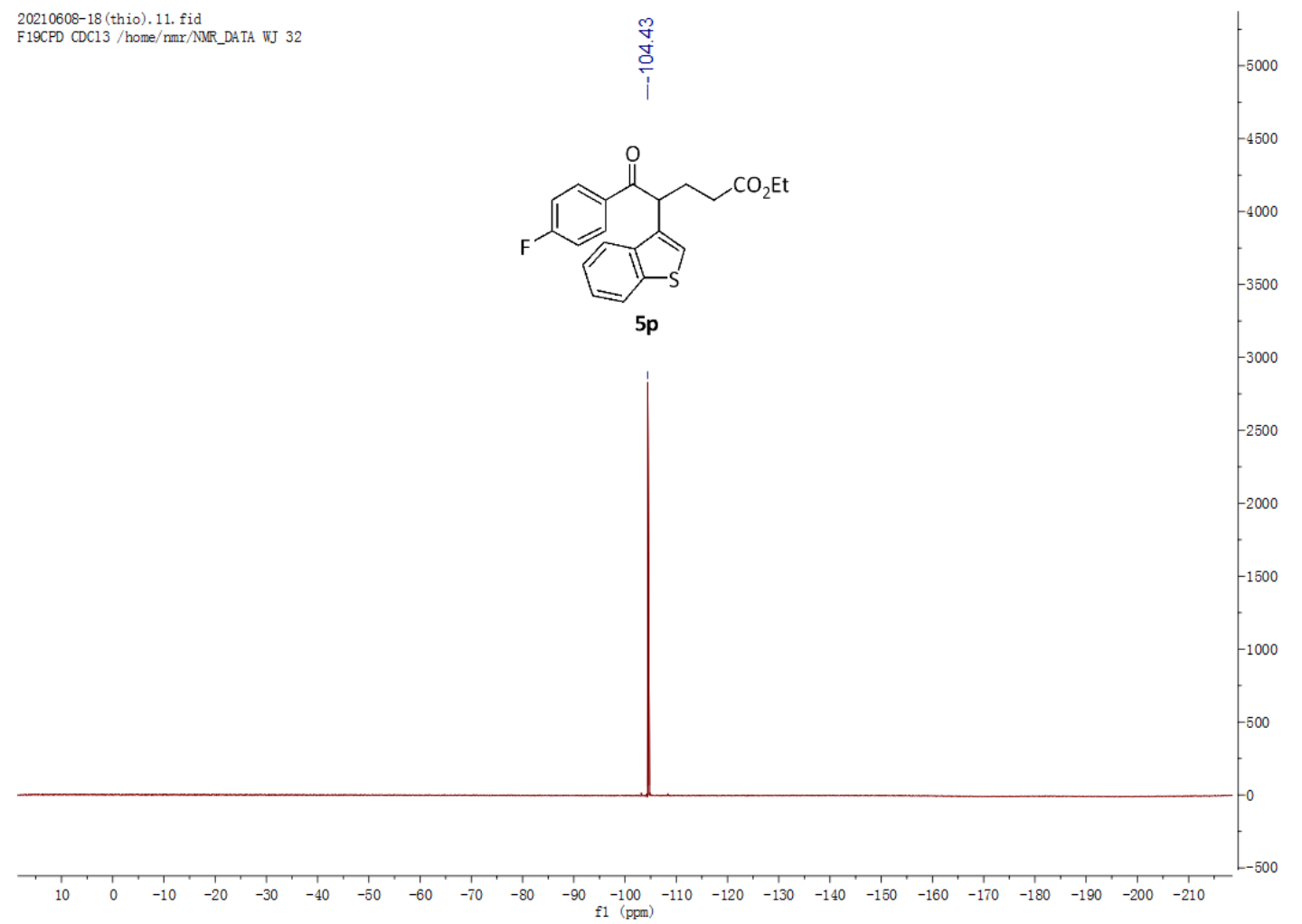
${ }^{13} \mathrm{C} \mathrm{NMR}\left(\mathrm{CDCl}_{3}, 100 \mathrm{MHz}\right)$

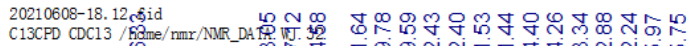

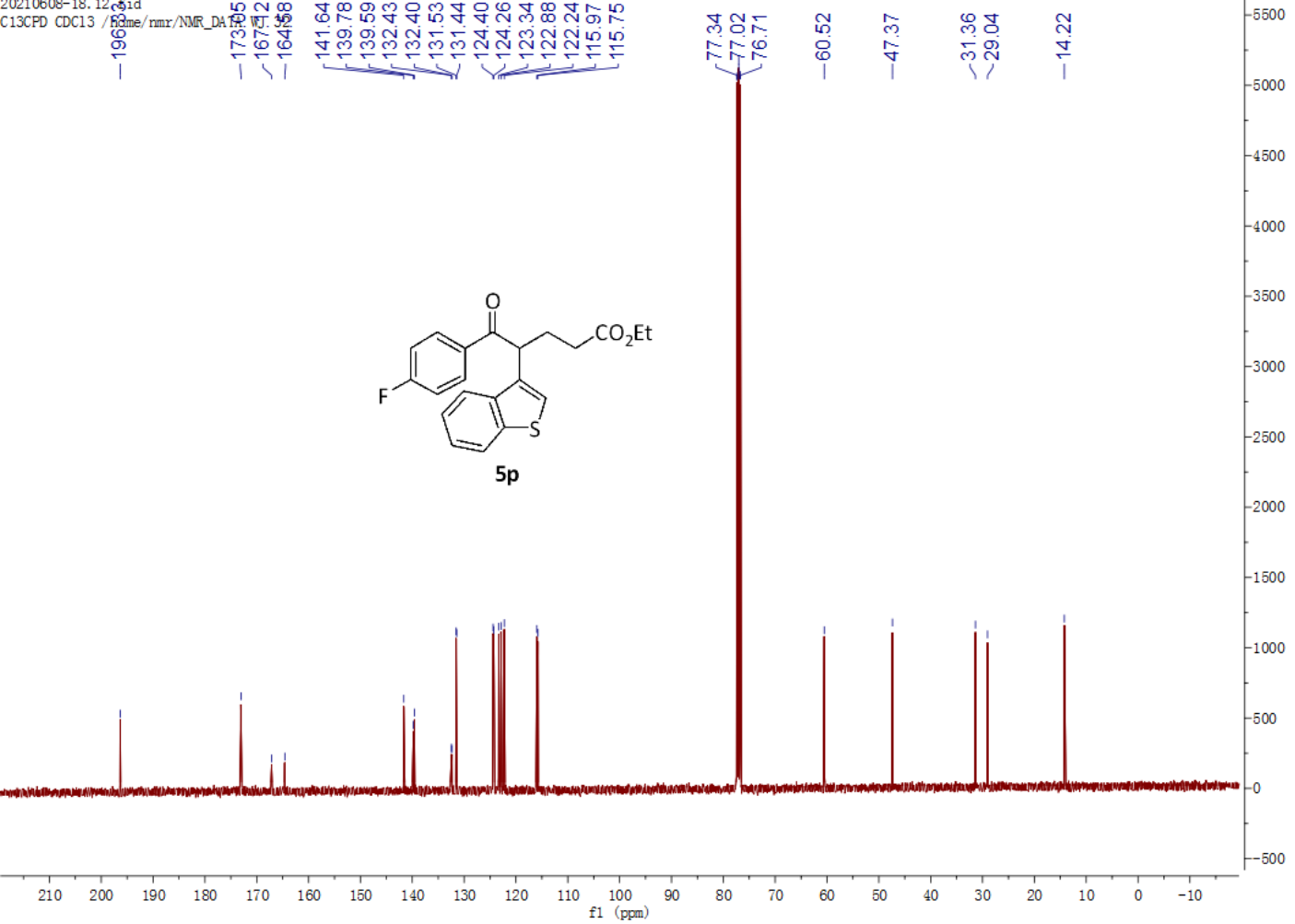




\section{Compound 6a}

${ }^{1} \mathrm{H} \mathrm{NMR}\left(\mathrm{CDCl}_{3}, 400 \mathrm{MHz}\right)$

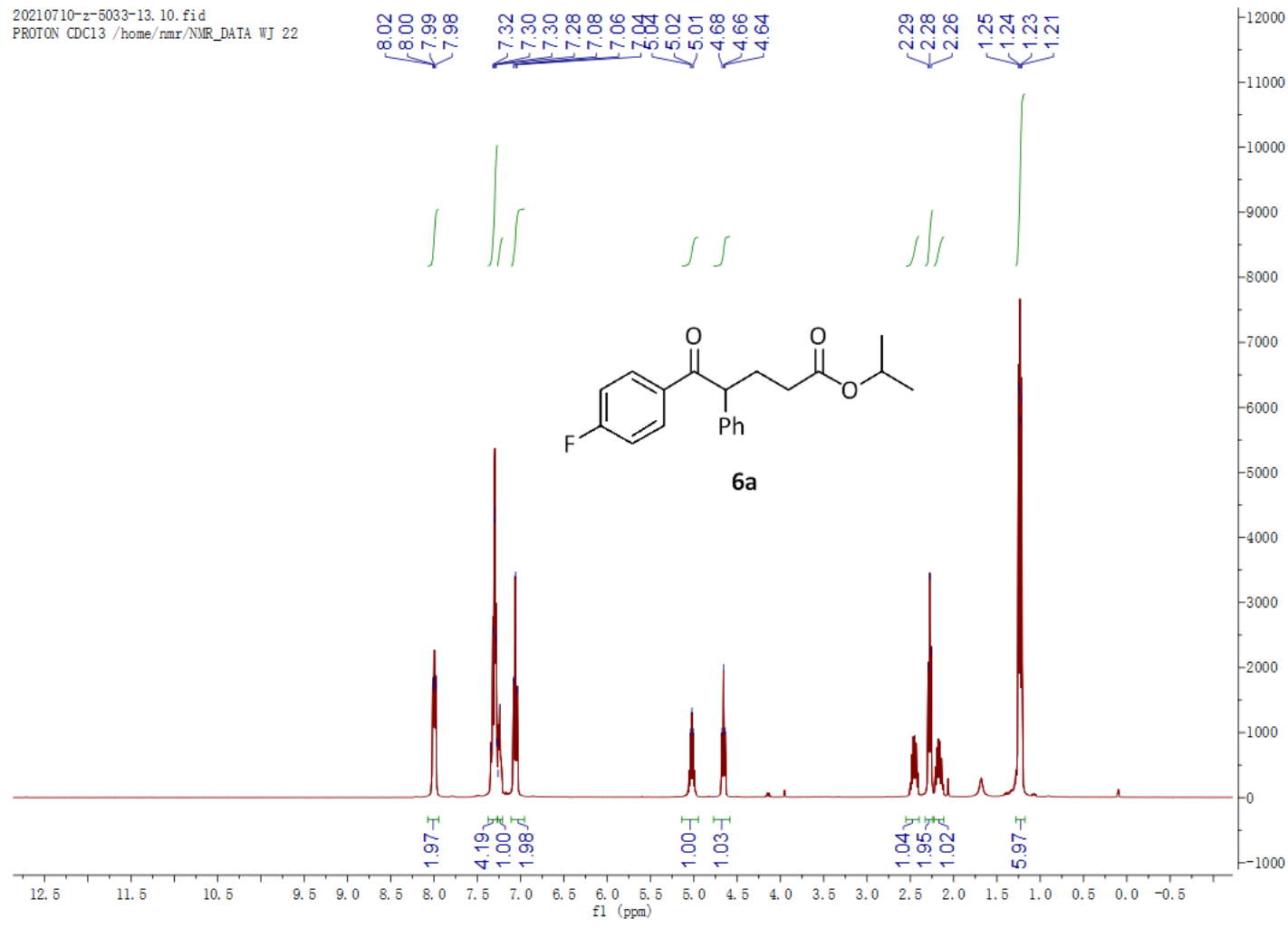

${ }^{19} \mathrm{~F} \mathrm{NMR}\left(\mathrm{CDCl}_{3}, 376 \mathrm{MHz}\right)$

20210710-z-5033-13. 11. fid
F19CPD CDC13 /home/nmr/NMR_DATA WJ 22
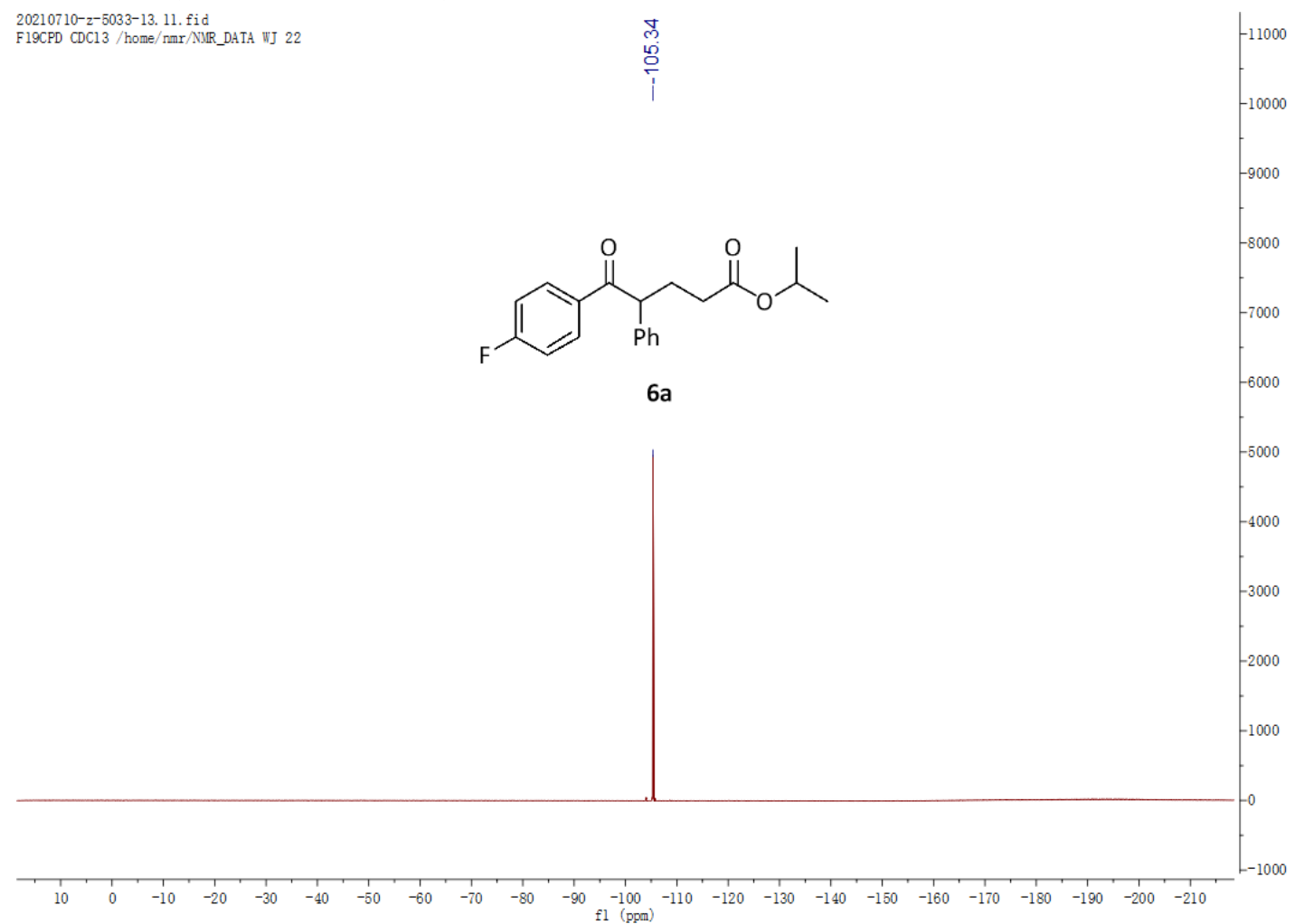
${ }^{13} \mathrm{C} \mathrm{NMR}\left(\mathrm{CDCl}_{3}, 100 \mathrm{MHz}\right)$

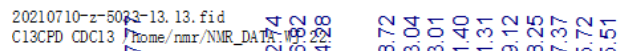

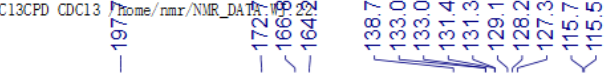

স

政舟

$\underset{\substack{i \\ i}}{i}$

두요요

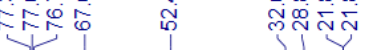

$-6500$

$-6000$

5500<smiles>CC(C)OC(=O)CCC(C(=O)c1ccc(F)cc1)c1ccccc1</smiles>

$6 a$

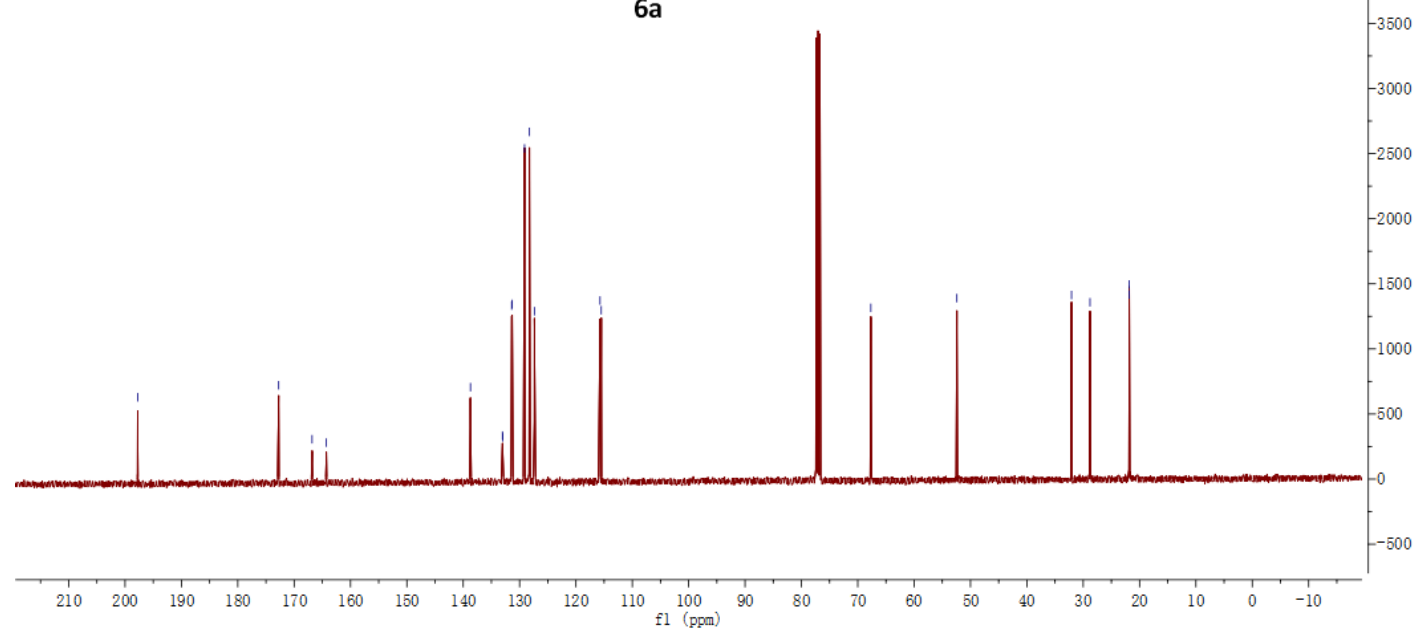




\section{Compound $\mathbf{6 b}$}

${ }^{1} \mathrm{H} \mathrm{NMR}\left(\mathrm{CDCl}_{3}, 400 \mathrm{MHz}\right)$

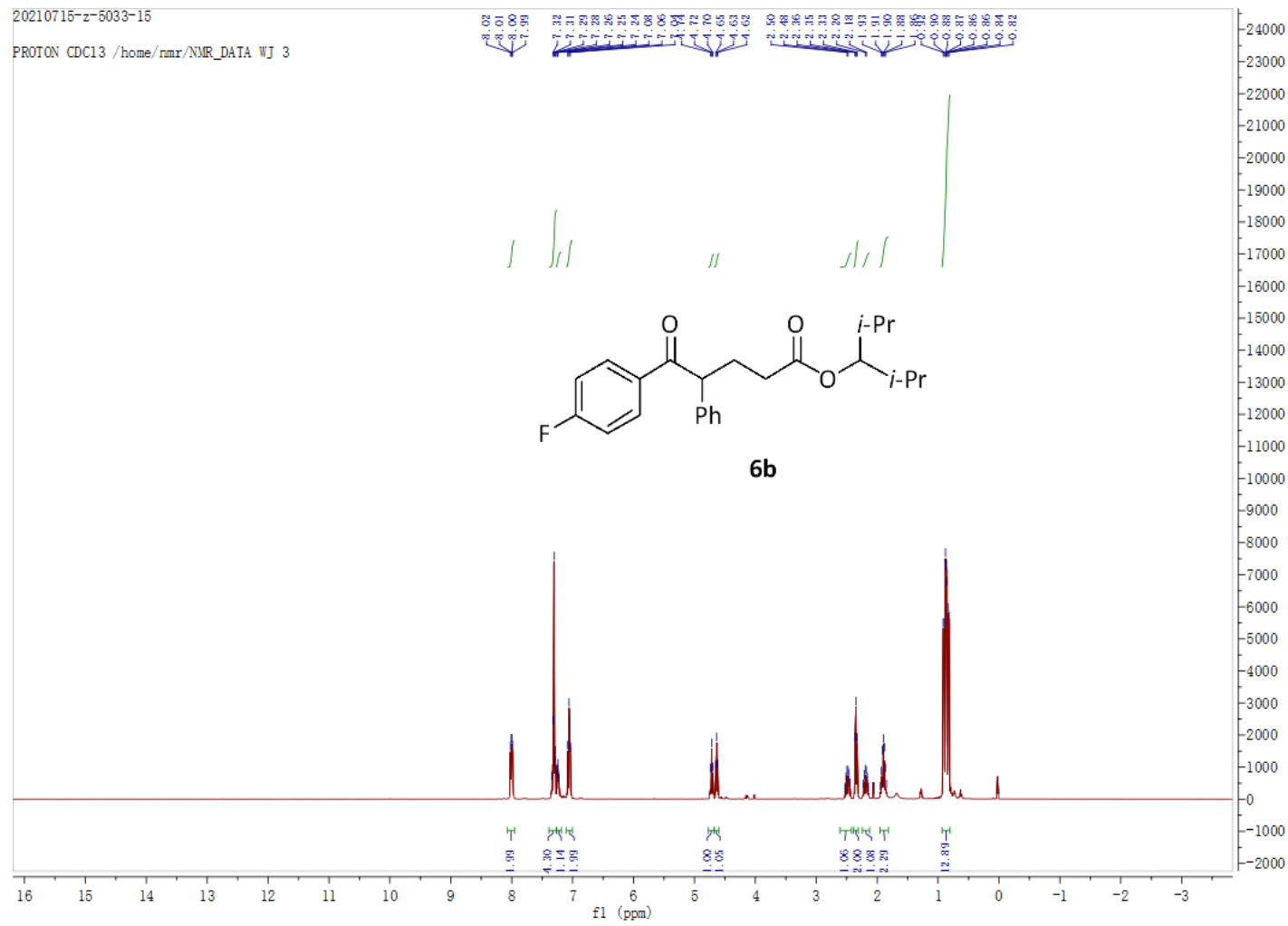

${ }^{19} \mathrm{~F} \mathrm{NMR}\left(\mathrm{CDCl}_{3}, 376 \mathrm{MHz}\right)$

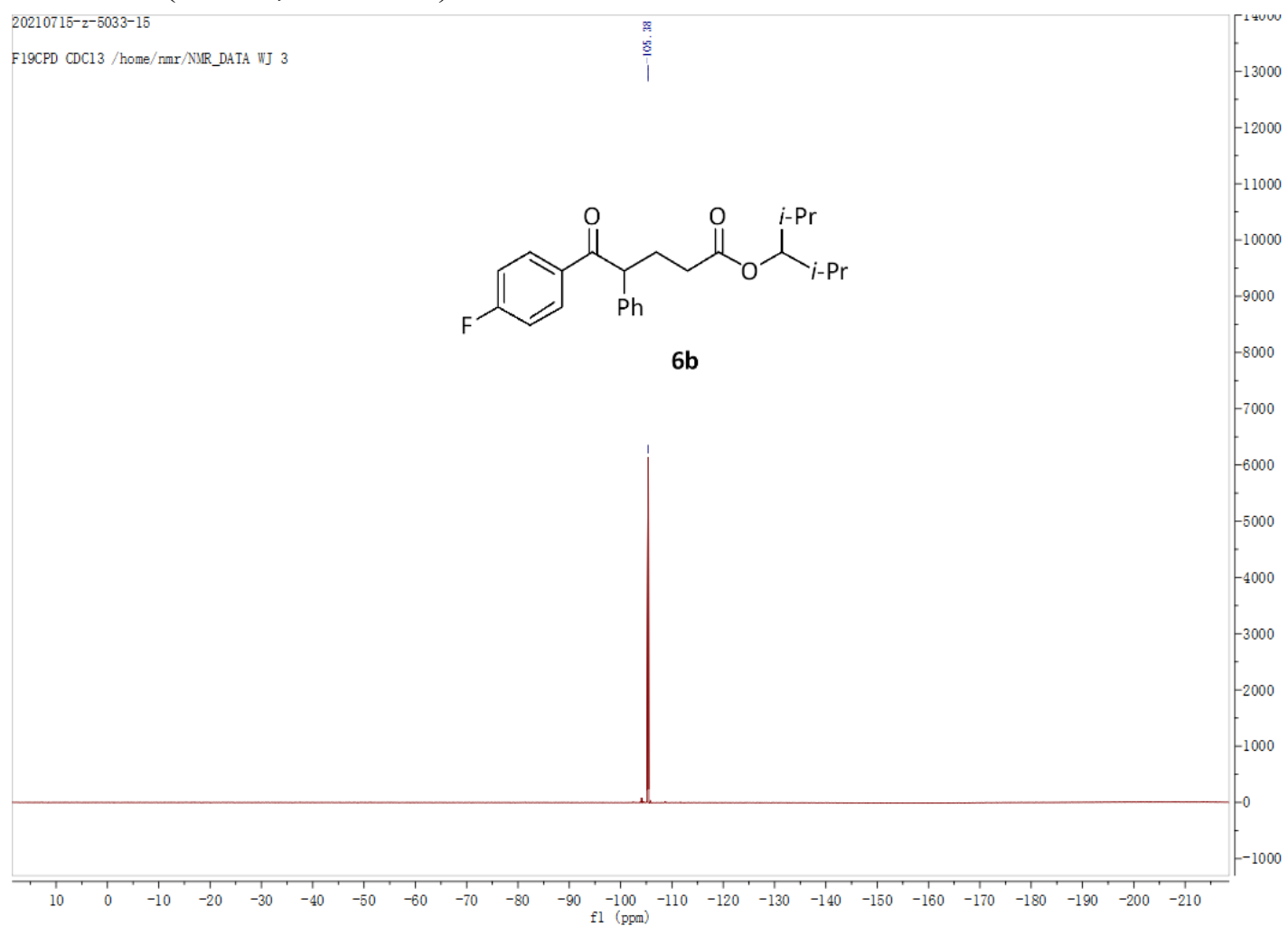


${ }^{13} \mathrm{C} \mathrm{NMR}\left(\mathrm{CDCl}_{3}, 100 \mathrm{MHz}\right)$

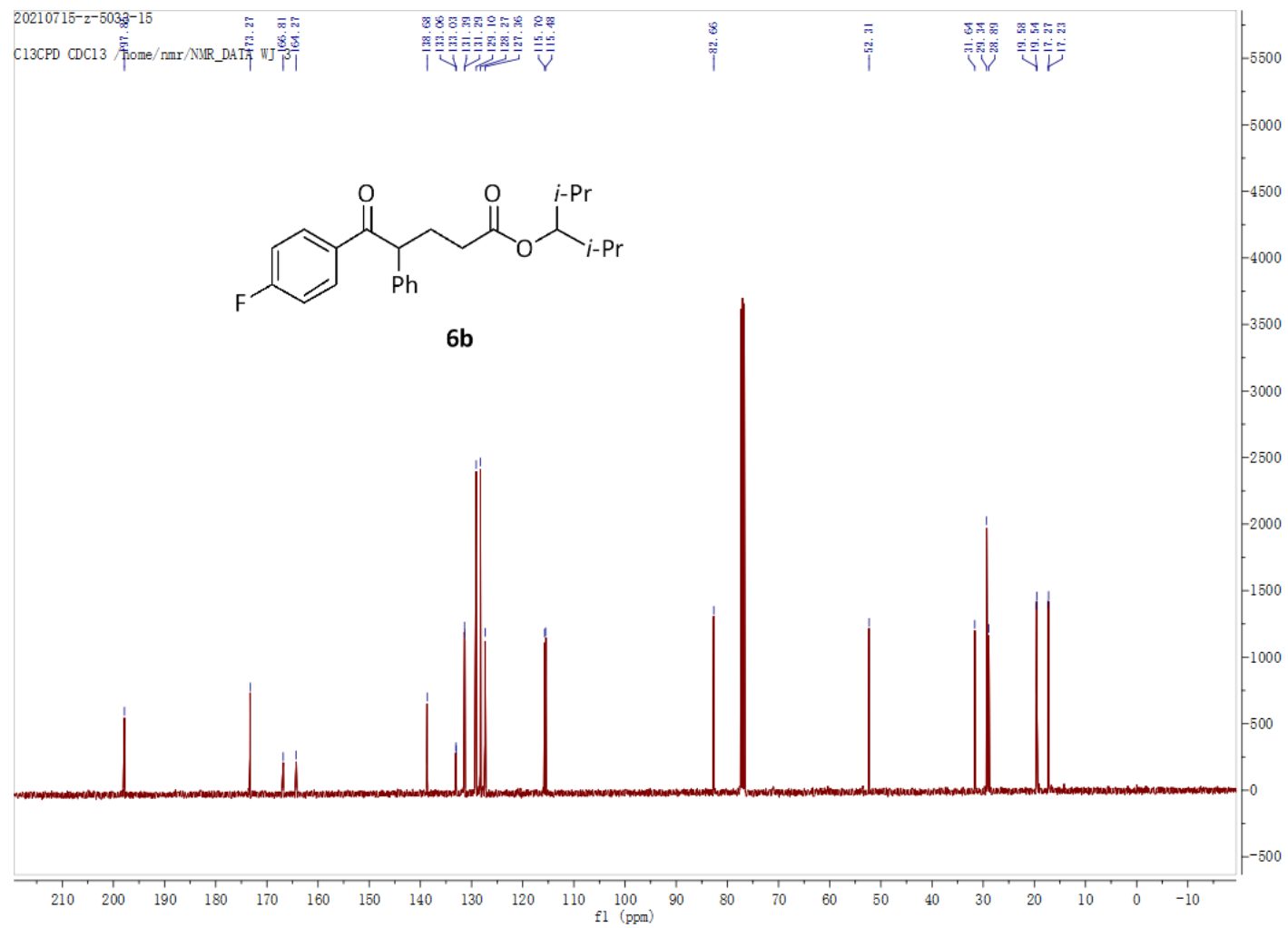




\section{Compound 6c}

${ }^{1} \mathrm{H} \mathrm{NMR}\left(\mathrm{CDCl}_{3}, 400 \mathrm{MHz}\right)$

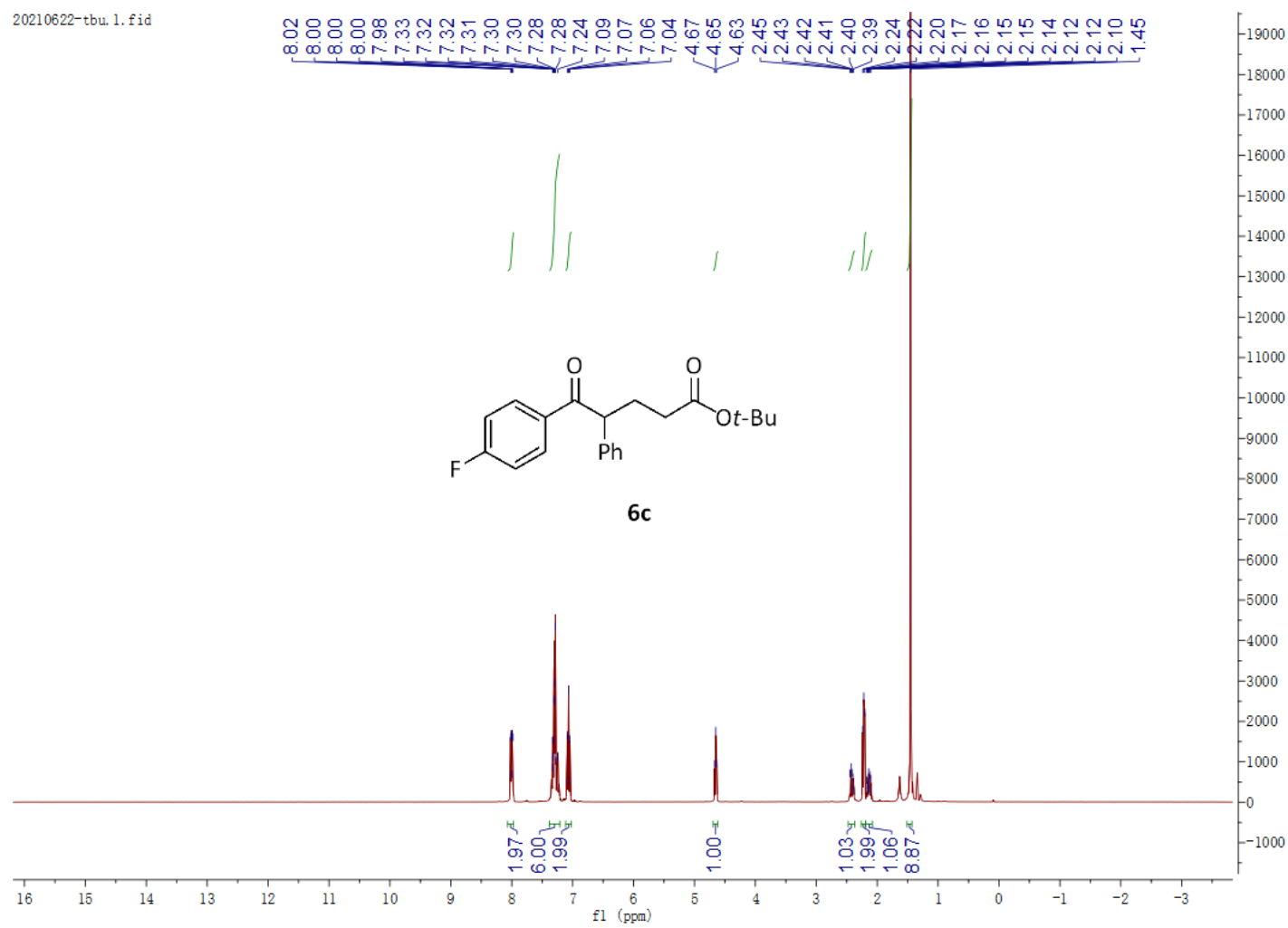

${ }^{19} \mathrm{~F} \mathrm{NMR}\left(\mathrm{CDCl}_{3}, 376 \mathrm{MHz}\right)$

20210622-tbu. 2. fid
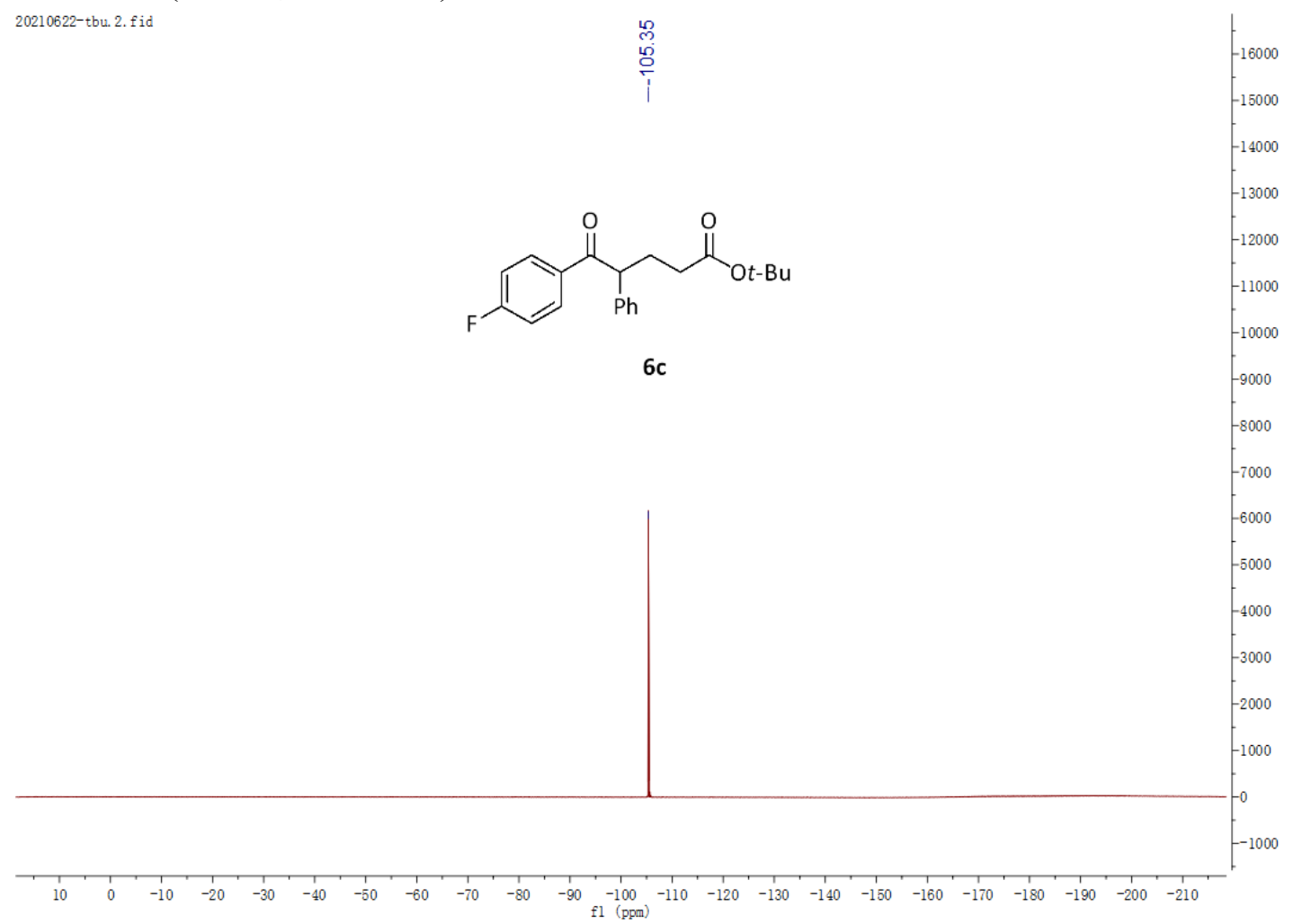
${ }^{13} \mathrm{C} \mathrm{NMR}\left(\mathrm{CDCl}_{3}, 100 \mathrm{MHz}\right)$

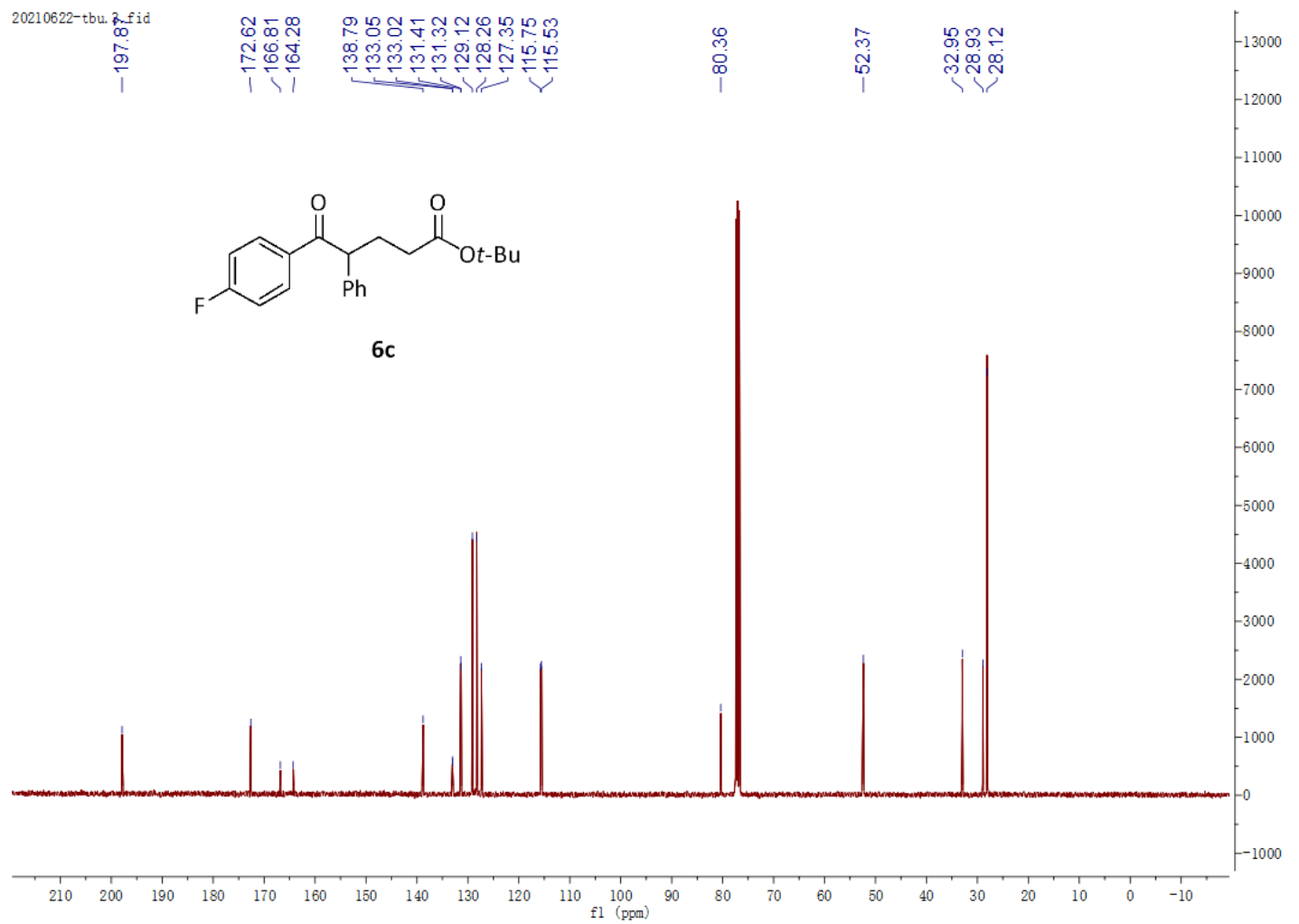




\section{Compound 6d}

${ }^{1} \mathrm{H} \mathrm{NMR}\left(\mathrm{CDCl}_{3}, 400 \mathrm{MHz}\right)$

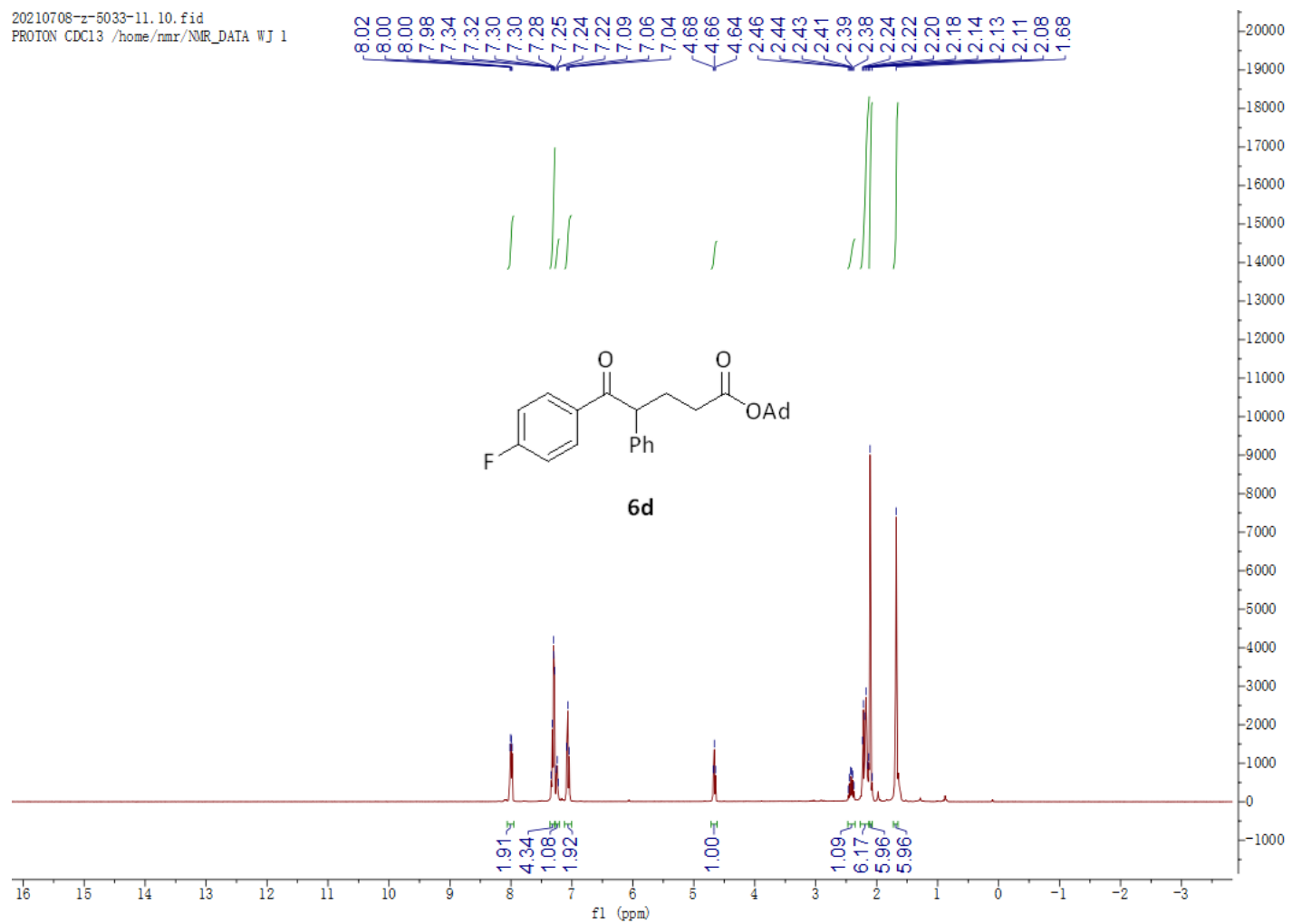

${ }^{19} \mathrm{~F} \mathrm{NMR}\left(\mathrm{CDCl}_{3}, 376 \mathrm{MHz}\right)$

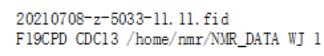
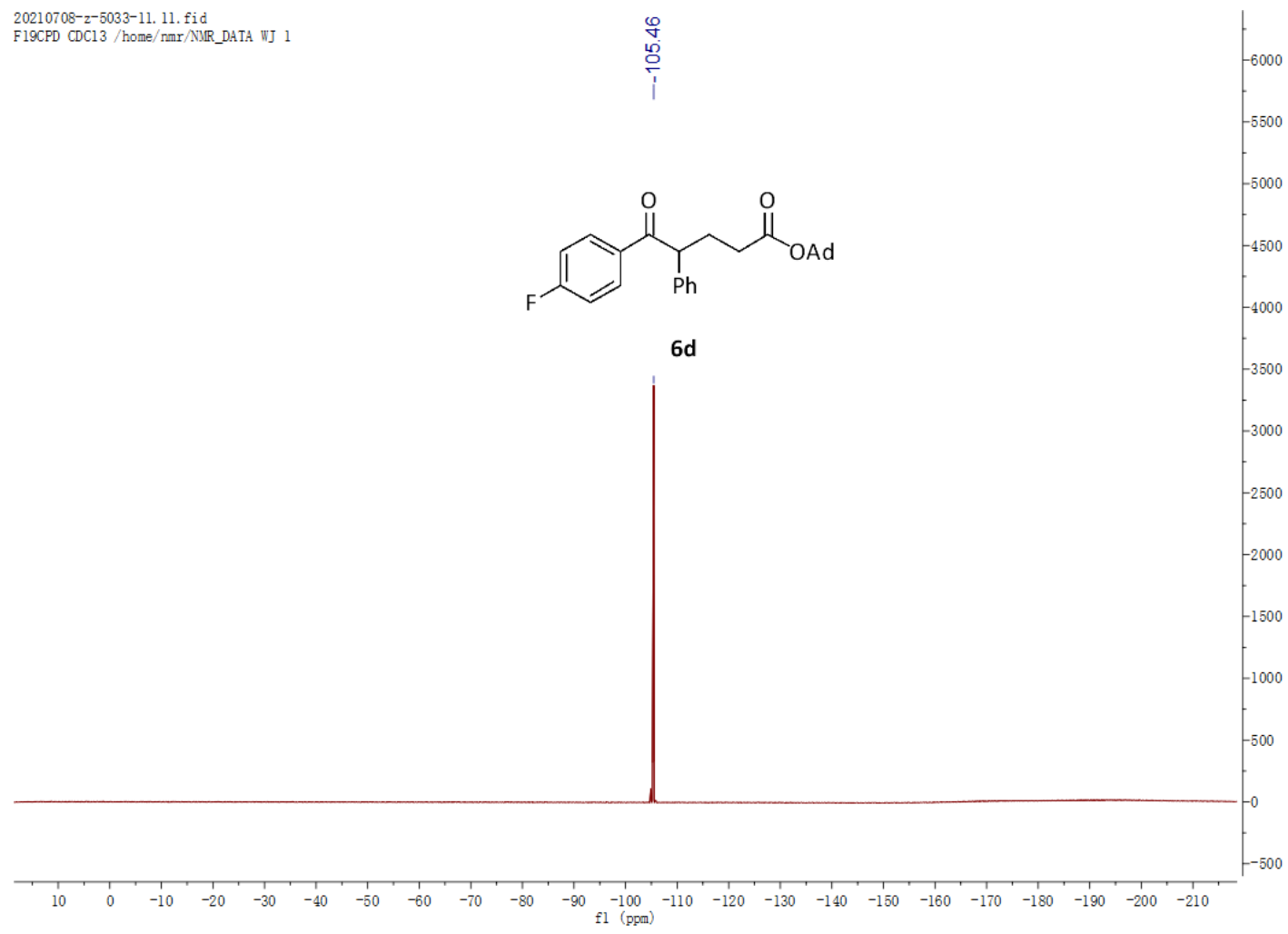

(1) 
${ }^{13} \mathrm{C} \mathrm{NMR}\left(\mathrm{CDCl}_{3}, 100 \mathrm{MHz}\right)$

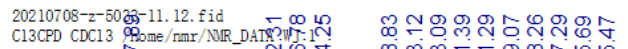

C13CPD CDC13

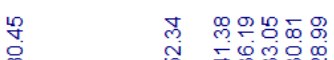

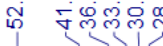

$-11000$

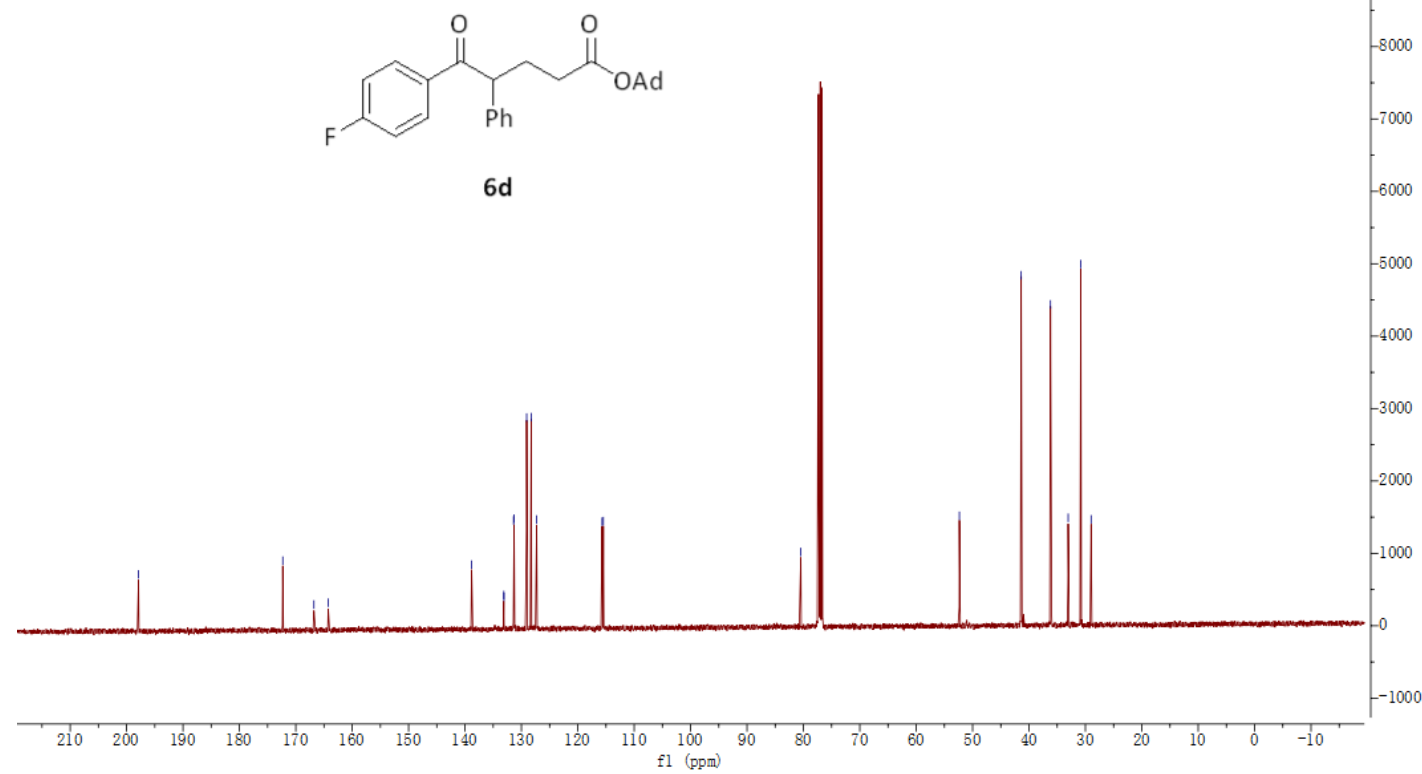

$6 d$ 


\section{Compound $\mathbf{6 e}$}

${ }^{1} \mathrm{H} \mathrm{NMR}\left(\mathrm{CDCl}_{3}, 400 \mathrm{MHz}\right)$

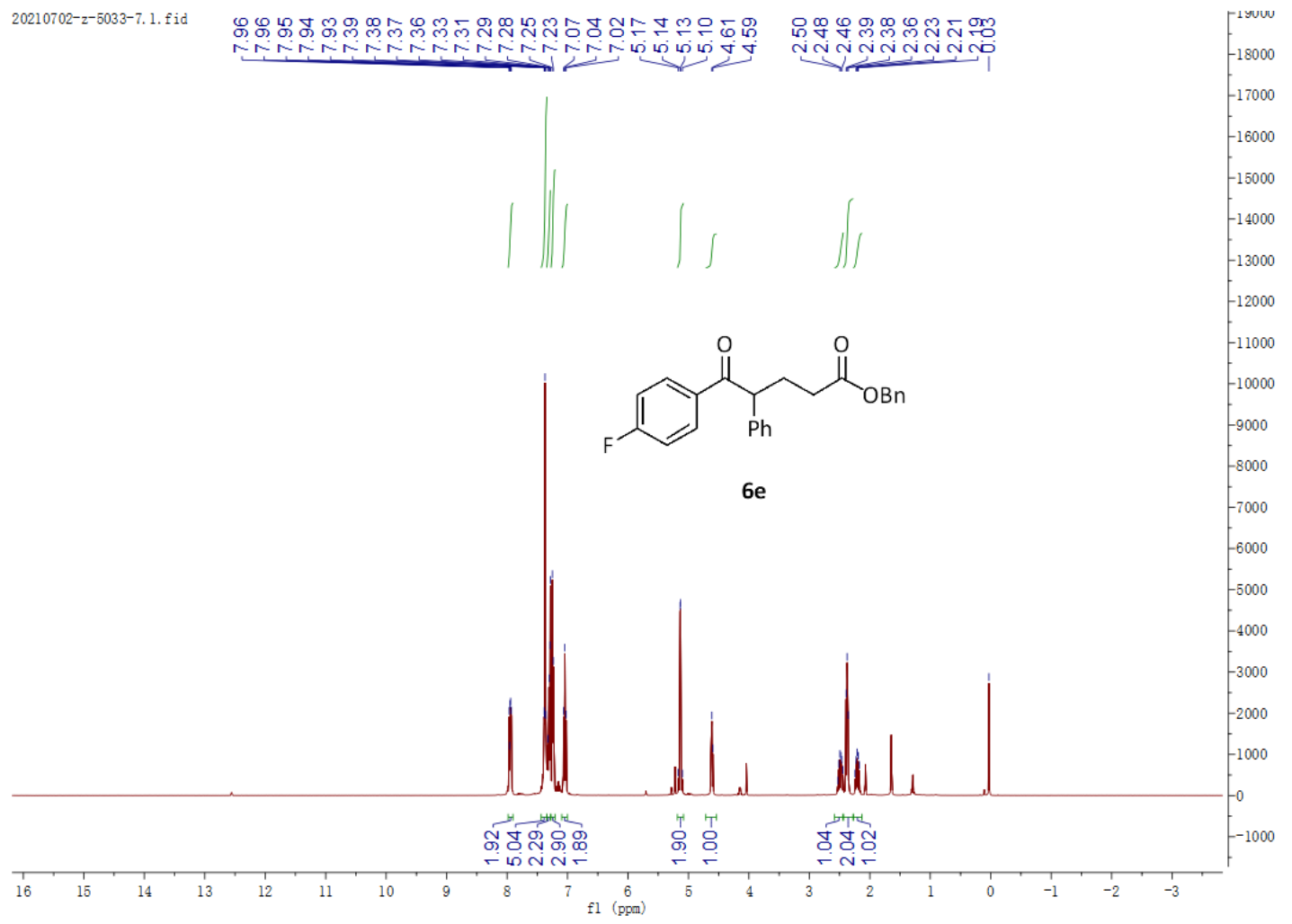

${ }^{19} \mathrm{~F} \mathrm{NMR}\left(\mathrm{CDCl}_{3}, 376 \mathrm{MHz}\right)$

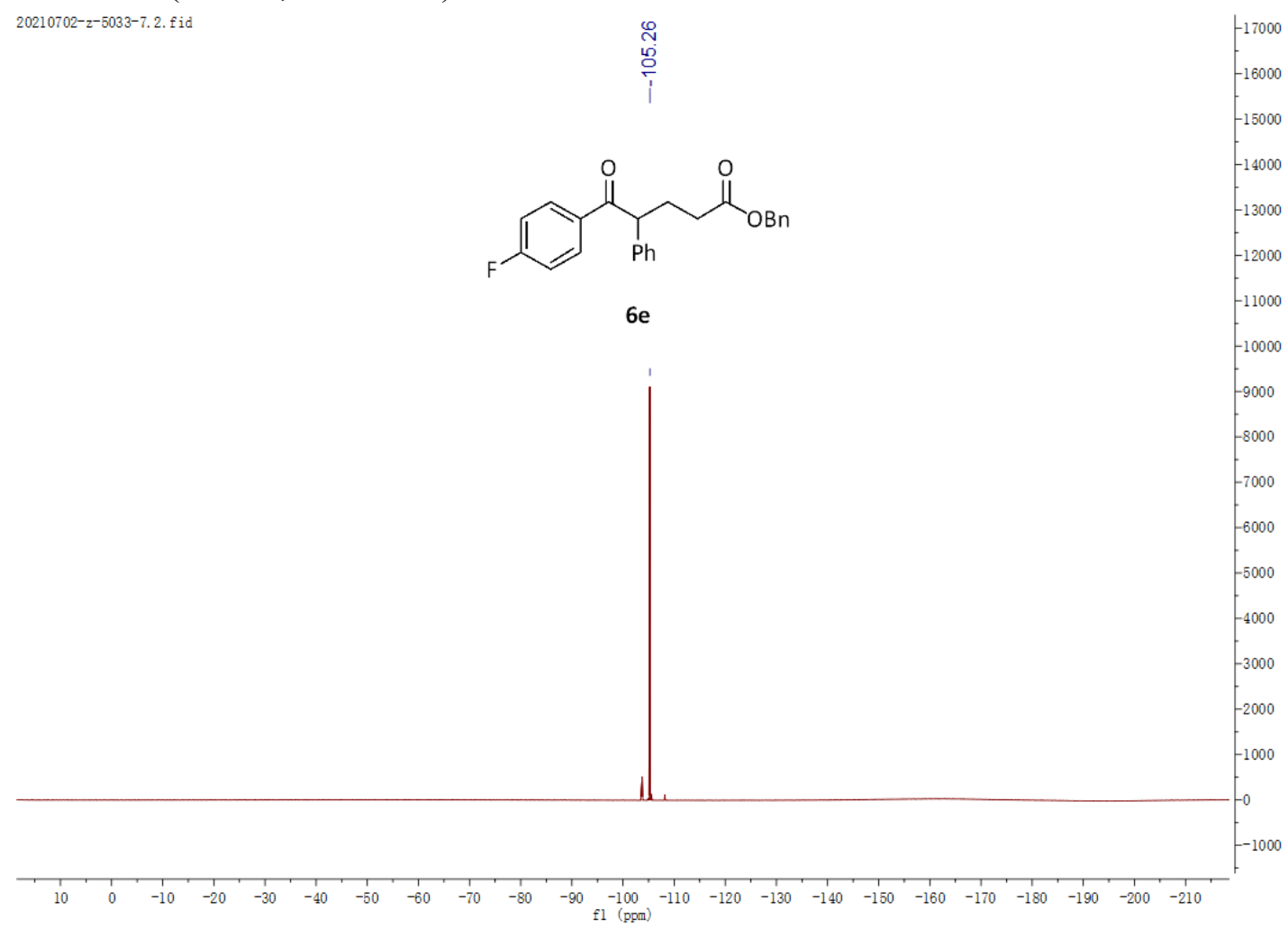


${ }^{13} \mathrm{C} \mathrm{NMR}\left(\mathrm{CDCl}_{3}, 100 \mathrm{MHz}\right)$

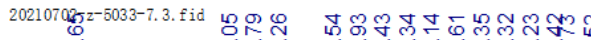

每

的果然

紫少

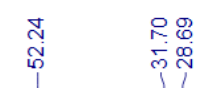

$-2000$<smiles>O=C(O)CCC(C(=O)c1ccccc1)c1ccc(F)cc1</smiles>

6e

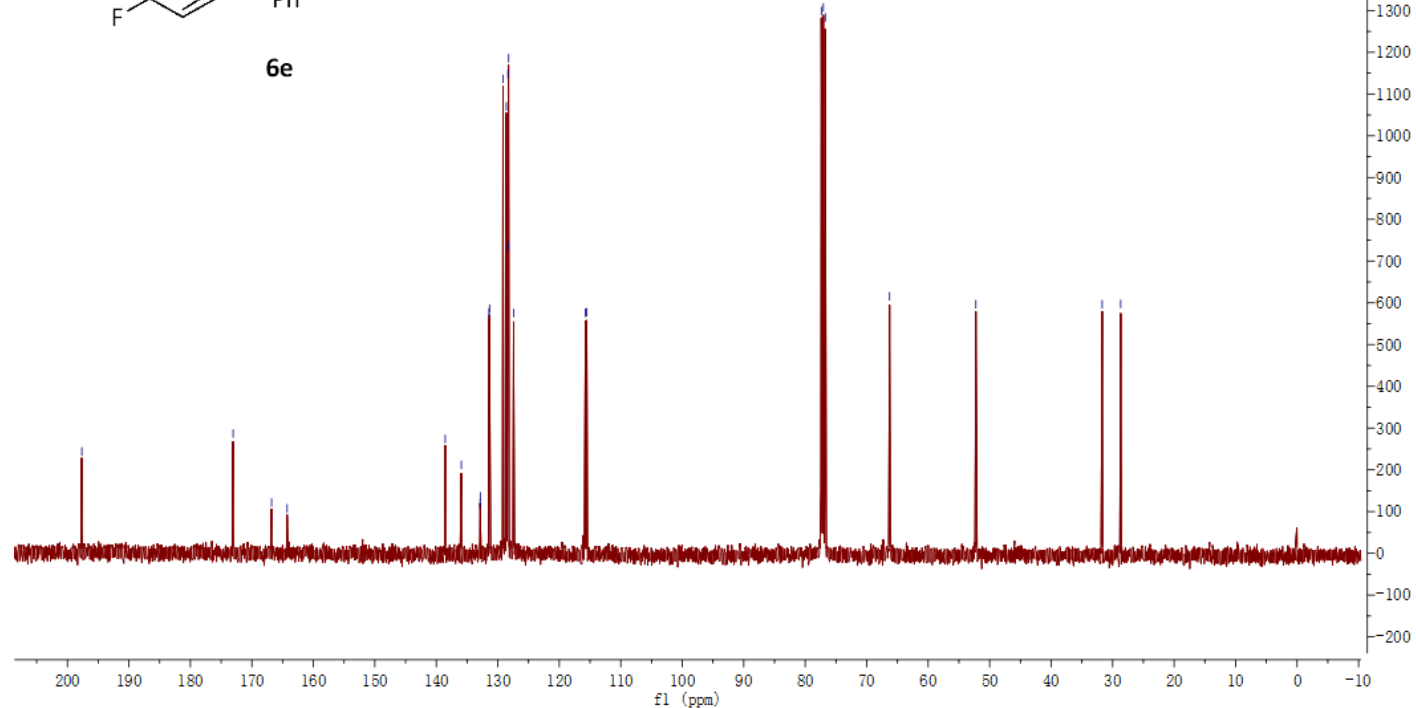




\section{Compound $\mathbf{6 f}$}

${ }^{1} \mathrm{H} \mathrm{NMR}\left(\mathrm{CDCl}_{3}, 400 \mathrm{MHz}\right)$

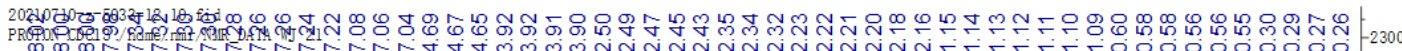

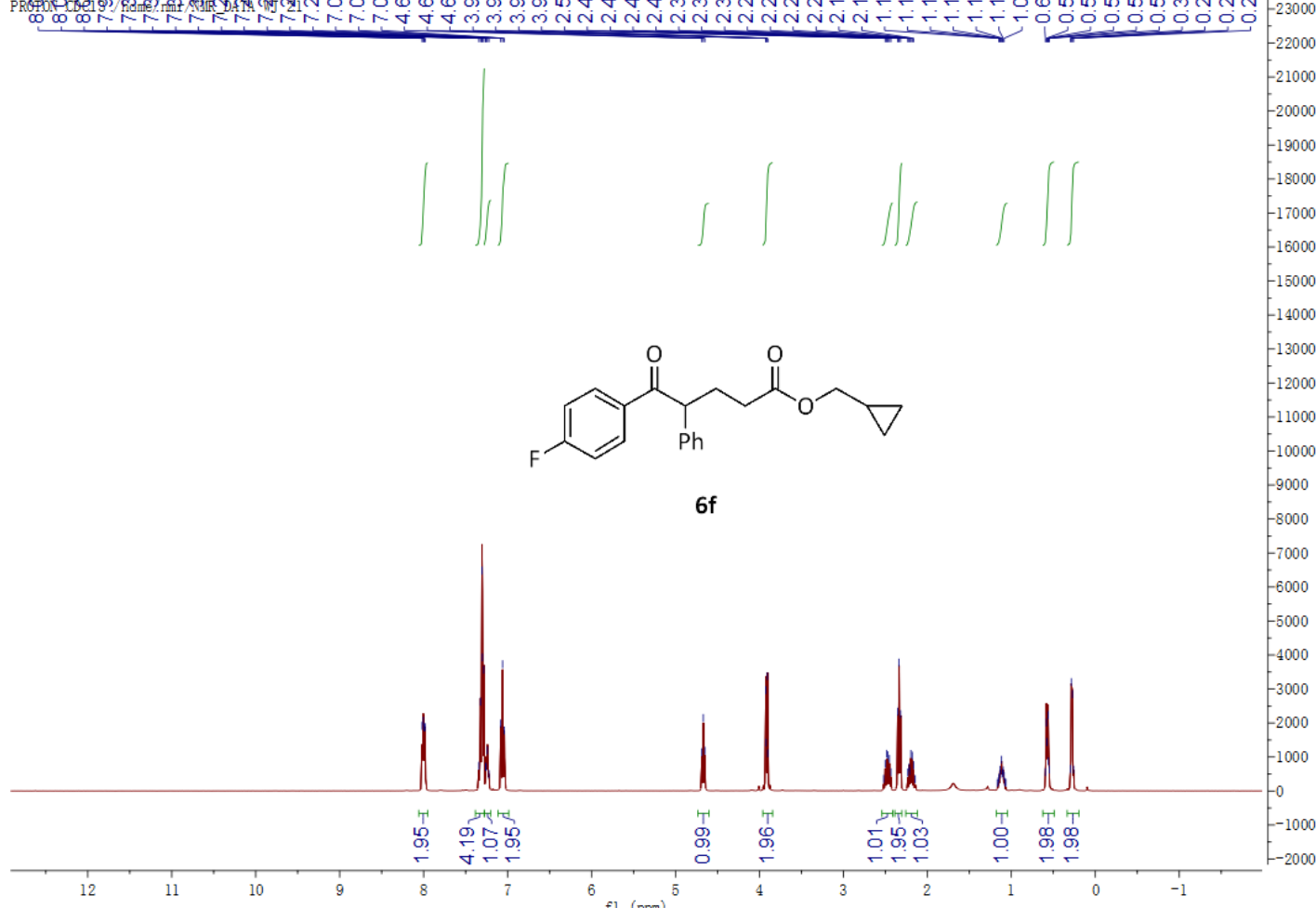

${ }^{19} \mathrm{~F} \mathrm{NMR}\left(\mathrm{CDCl}_{3}, 376 \mathrm{MHz}\right)$

20210709- $z-5033-12.11$. fid
F19CPD CDC13/home/rmm/NIR_DATA WI 2

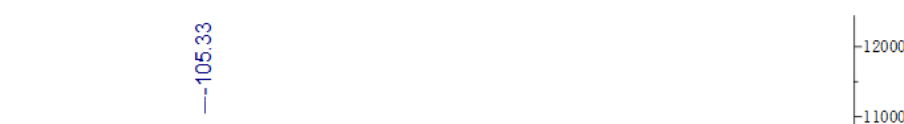<smiles>O=C(CCC(C(=O)c1ccc(F)cc1)c1ccccc1)OCC1CC1</smiles>

$6 f$

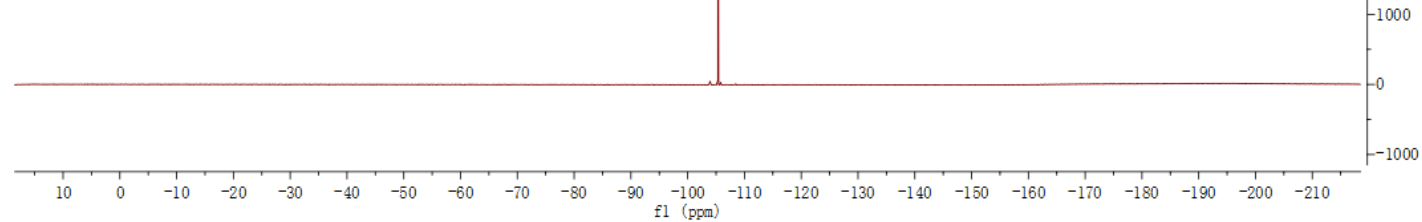


${ }^{13} \mathrm{C} \mathrm{NMR}\left(\mathrm{CDCl}_{3}, 100 \mathrm{MHz}\right)$

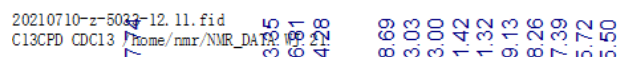

I

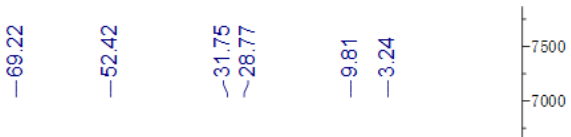

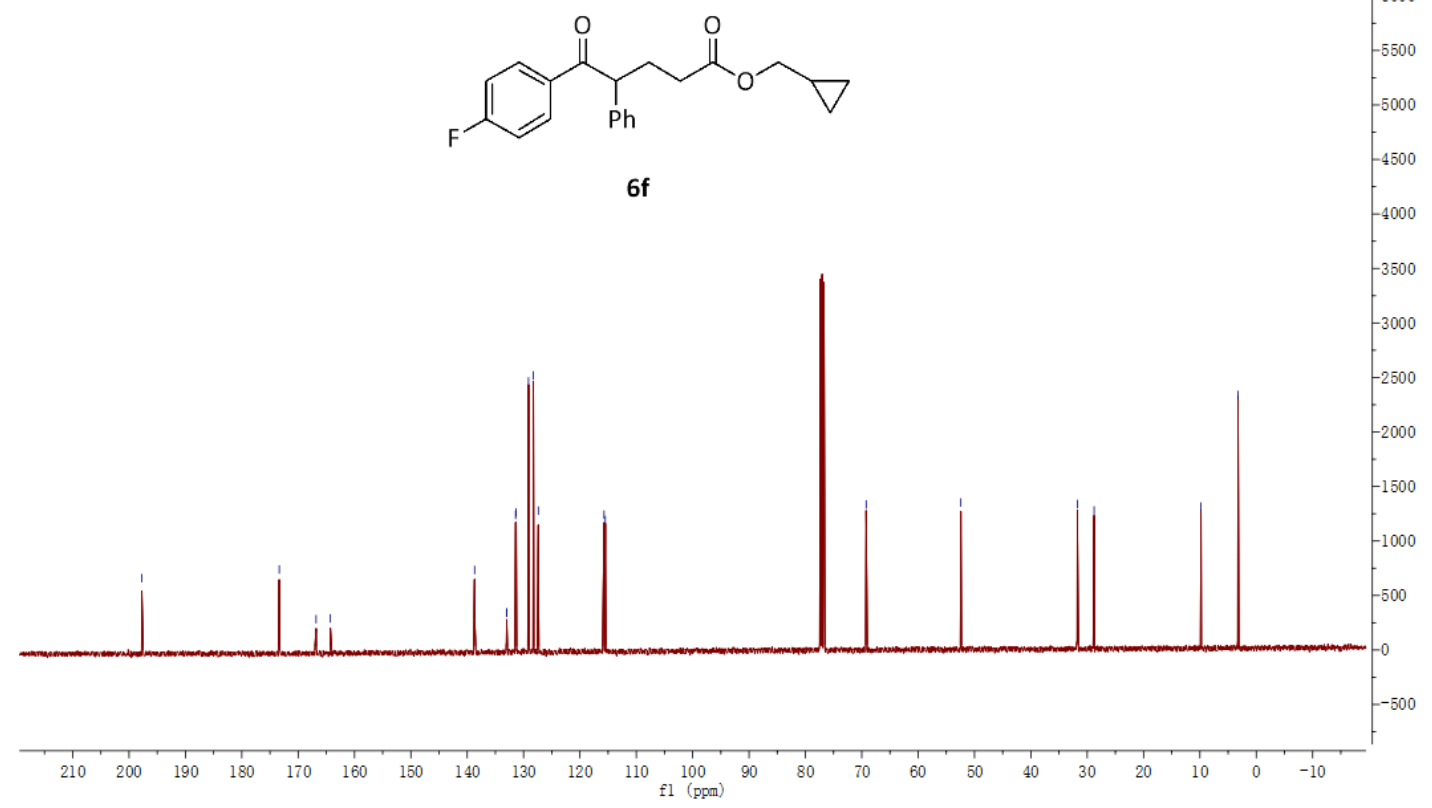

$6 f$ 


\section{Compound 69}

${ }^{1} \mathrm{H} \mathrm{NMR}\left(\mathrm{CDCl}_{3}, 400 \mathrm{MHz}\right)$

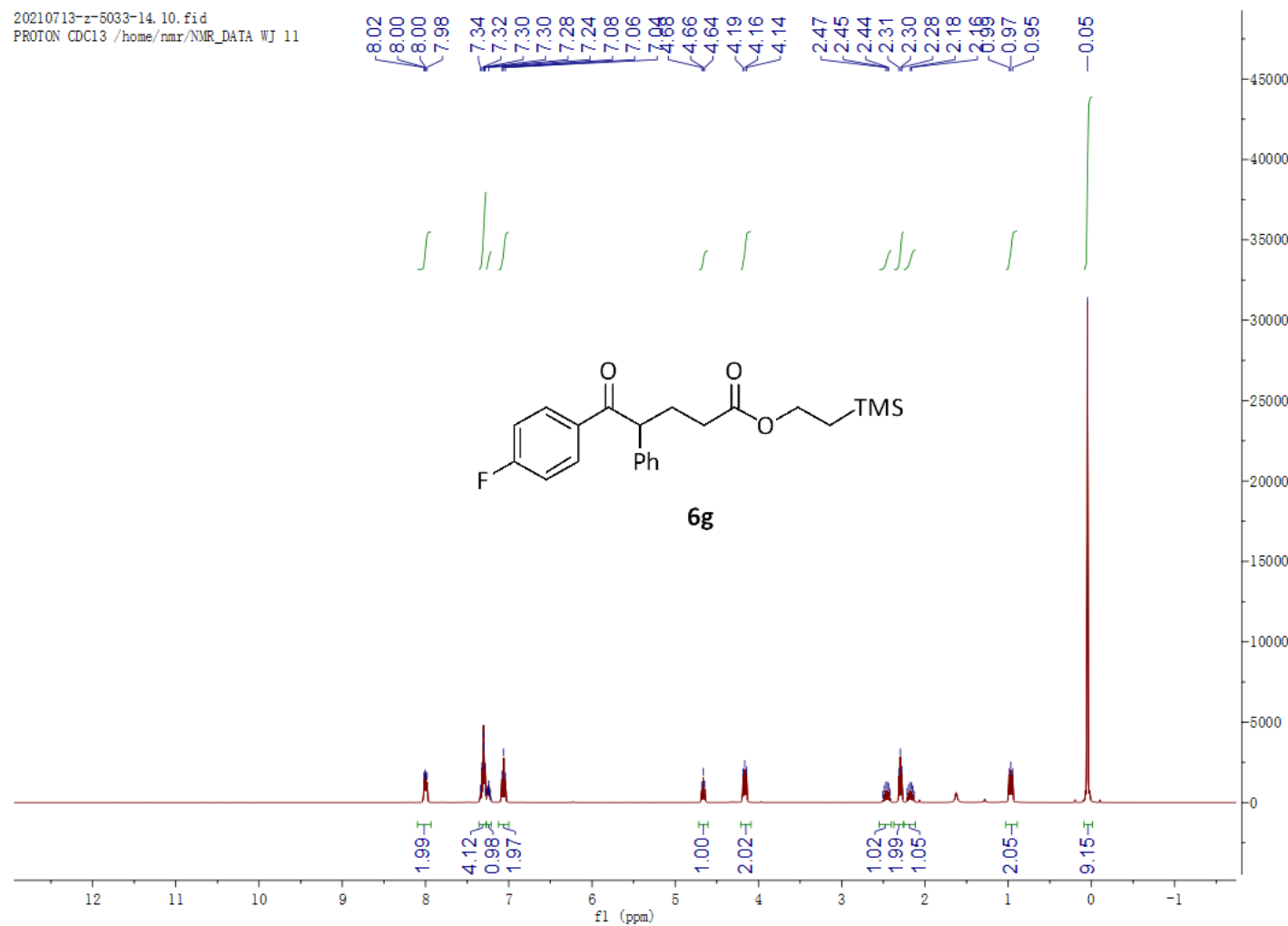

${ }^{19} \mathrm{~F} \mathrm{NMR}\left(\mathrm{CDCl}_{3}, 376 \mathrm{MHz}\right)$

20210713-z-5033-14. 11. fid
F19CPD CDC13/home/rmr/NIR_DATA WI 11

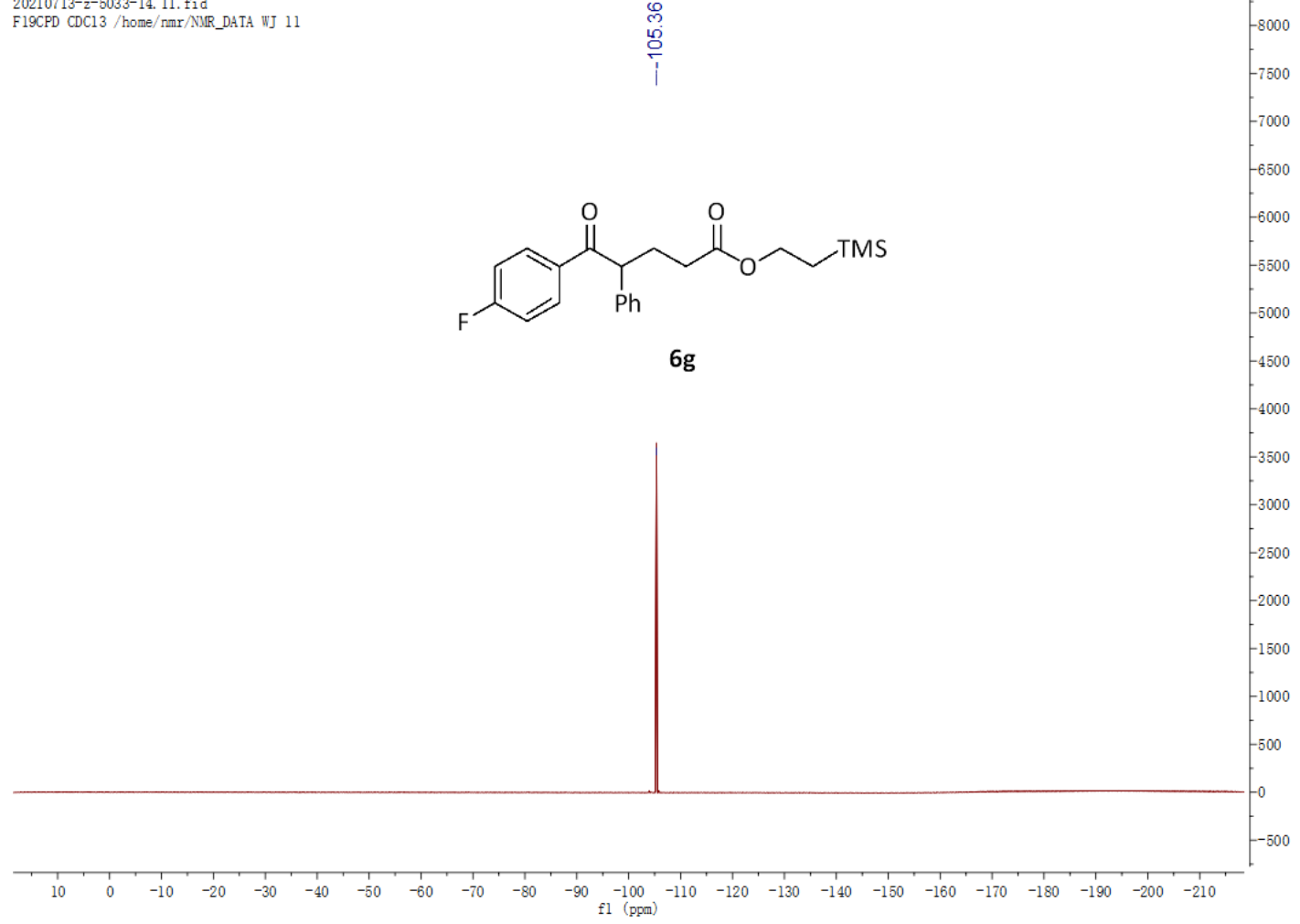


${ }^{13} \mathrm{C} \mathrm{NMR}\left(\mathrm{CDCl}_{3}, 100 \mathrm{MHz}\right)$

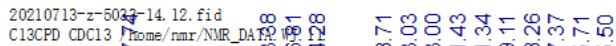

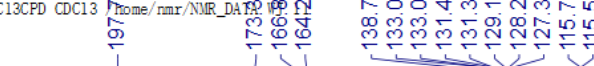<smiles>CS(=O)(=O)CCOC(=O)CCC(C(=O)c1ccc(F)cc1)c1ccccc1</smiles>

$6 \mathrm{~g}$

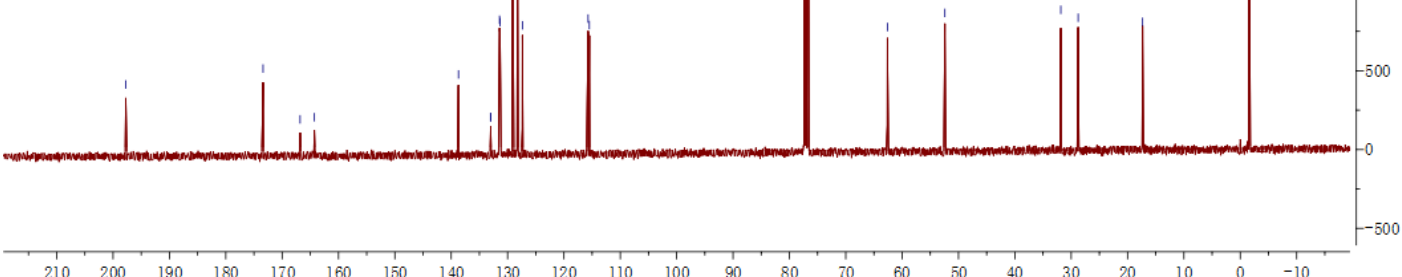

紫

$-4500$

$-4000$

3500

5000

2000 